\title{
Preventive genetic testing
}

Citation for published version (APA):

Stewart, K. F. J. (2018). Preventive genetic testing: our best foot forward. [Doctoral Thesis, Maastricht University]. Maastricht University. https://doi.org/10.26481/dis.20180920ks

Document status and date:

Published: 01/01/2018

DOI:

10.26481/dis.20180920ks

Document Version:

Publisher's PDF, also known as Version of record

\section{Please check the document version of this publication:}

- A submitted manuscript is the version of the article upon submission and before peer-review. There can be important differences between the submitted version and the official published version of record.

People interested in the research are advised to contact the author for the final version of the publication, or visit the DOI to the publisher's website.

- The final author version and the galley proof are versions of the publication after peer review.

- The final published version features the final layout of the paper including the volume, issue and page numbers.

Link to publication

\footnotetext{
General rights rights.

- You may freely distribute the URL identifying the publication in the public portal. please follow below link for the End User Agreement:

www.umlib.nl/taverne-license

Take down policy

If you believe that this document breaches copyright please contact us at:

repository@maastrichtuniversity.nl

providing details and we will investigate your claim.
}

Copyright and moral rights for the publications made accessible in the public portal are retained by the authors and/or other copyright owners and it is a condition of accessing publications that users recognise and abide by the legal requirements associated with these

- Users may download and print one copy of any publication from the public portal for the purpose of private study or research.

- You may not further distribute the material or use it for any profit-making activity or commercial gain

If the publication is distributed under the terms of Article $25 \mathrm{fa}$ of the Dutch Copyright Act, indicated by the "Taverne" license above, 


\section{Preventive genetic testing \\ Our best foot forward}


(C) Kelly Stewart, Maastricht, 2018

Cover design \& layout: Stefanie van den Herik | SVDH Media Print: ProefschriftMaken

ISBN: 978-90-829118-0-0

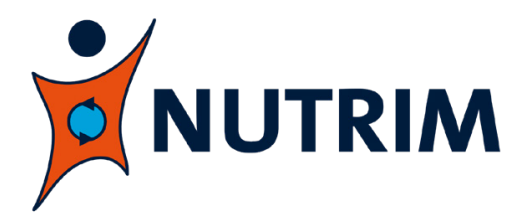

The research presented in this thesis was conducted at NUTRIM School of Nutrition and Translational Research in Metabolism 


\title{
Preventive genetic testing Our best foot forward
}

\author{
DISSERTATION
}

to obtain the degree of Doctor at the Maastricht University, on the authority of the Rector Magnificus, Prof. dr. Rianne M. Letschert

in accordance with the decision of the Board of Deans, to be defended in public

on Thursday 20 September 2018, at 16.00 hours

By

Kelly Frances Jeanne Stewart 


\section{Promotores}

Prof. dr. M.P.A. Zeegers

Prof. dr. A.M.W.J. Schols

\section{Co-promotor:}

Dr. A. Wesselius

\section{Assessment committee}

Prof. dr. S.A. Morré (chair)

Dr. S. Braun (Hogeschool Zuyd)

Prof. dr. S.P.J. Kremers

Prof. Dr. H. Snieder (Universitair Medisch Centrum Groningen)

Prof. Dr. D.M.R. Townend 


\section{Contents}

Chapter 1 General introduction

Chapter 2 Factors associated with acceptability, consideration and 17 intention of uptake of direct-to-consumer genetic testing: a survey study

Chapter 3 Genetic, lifestyle, and environmental factors associated with 43 development of COPD - A systematic review of meta-analyses

Chapter 4 Behavioural changes, sharing behaviour and psychological 123 responses after receiving direct-to-consumer genetic test results: a systematic review and meta-analysis

Chapter 5 Modifiable risk factors for the prevention of bladder cancer: 167 a systematic review of meta-analyses

Chapter 6 Stages of behavioural change after direct-to-consumer disease risk profiling: study protocol of two integrated controlled pragmatic trials

Chapter 7 General discussion

Summary

Samenvatting

Valorisation

Dankwoord

About the author 



\section{Manuscripts forming the basis of this thesis}

Chapter 2

Stewart KFJ, Kokole D, Wesselius A, Schols AMWJ, Zeegers MP, Van Osch LADM. Factors associated with acceptability, consideration and intention of uptake of direct-toconsumer genetic testing: a survey study. (accepted by Public Health Genomics)

Chapter 3

Mount SW*, Stewart KFJ*, Wesselius A, Zeegers MP, Schols AMWJ. Genetic, lifestyle, and environmental factors associated with development of chronic obstructive pulmonary disease. (submitted)

\section{Chapter 4}

Stewart KFJ, Wesselius A, Schreurs MA, Schols AM, Zeegers MP. Behavioural changes, sharing behaviour and psychological responses after receiving direct-to-consumer genetic test results: a systematic review and meta-analysis. Journal of community genetics. 2017 Jun 29:1-8.

\section{Chapter 5}

Al-Zalabani AH*, Stewart KFJ*, Wesselius A, Schols AM, Zeegers MP. Modifiable risk factors for the prevention of bladder cancer: a systematic review of meta-analyses. European journal of epidemiology. 2016 Sep 1;31(9):811-51.

\section{Chapter 6}

Stewart KFJ, Wesselius A, Schols A M W J, Zeegers MP. Stages of behavioural change after direct-to-consumer disease risk profiling: study protocol of two integrated controlled pragmatic trials. Trials Journal. 2018; 19(1):240. 


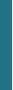




\section{Sicker people and poorer lifestyles}

The world is facing a very challenging epidemic of non-communicable diseases (NCD), which is not expected to improve. The majority of the 40 million deaths from NCDs worldwide in 2015 were caused by cardiovascular disease, cancer, chronic respiratory diseases and diabetes type II, and rates are expected to rise to 52 million deaths by $2030 .^{1,2}$ These developments will impact quality of life of affected individuals and their social environment and will carry an economic burden for society as a whole. However, in addition to genetic susceptibilities, important causes of these chronic diseases are poor lifestyle habits and related factors, such as tobacco and alcohol use, poor diet or physical inactivity, and high blood pressure or blood glucose levels. ${ }^{3}$ As many of the risk factors are modifiable to a greater or lesser extent, this encourages exploration of preventive strategies.

\section{Changing behaviour because of increased disease risk?}

One such approach could be through learning of an increased disease risk. It has been hypothesised that if one learns to be at an increased risk of disease, this might trigger individuals to change their behaviour. ${ }^{4}$ For example, a smoking individual could consider quitting when they learn they are at an increased risk of lung cancer as a result of smoking. Although such a general recommendation as quitting smoking to reduce disease risk may certainly elicit positive behaviour change, including a very personal factor as one's genetic make-up into the risk prediction model may be even more triggering. One approach to personalising disease risk estimation through genetics is that of direct-to-consumer genetic testing (DTC-GT). This is a group of genetic testing services aiming to estimate common chronic disease risks based on an individual's genetic make-up. As the name suggests, DTC-GT is aimed directly at the individual and can thereby be taken up without any involvement of a health care professional (HCP). The entire process takes place online and via regular mail: the customer consents online, sends their DNA sample by mail after which the results are returned in an online report. In addition, information on interpretation of the results is mostly provided online too, sometimes supplemented with optional genetic counselling by telephone.

\section{Introduction to the debate}

Currently, the momentum for DTC-GT is large and increasing. When in 2013 the United States Food and Drug Authority (FDA) banned one of the largest and very 
popular DTC-GT company 23andMe of selling their health-related genetic services, the debate has particularly fired up. ${ }^{5}$ By 2017 , tests for more than ten diseases were approved by the FDA, as the company had shown that the results were consistent and reliable, saliva collection was easy enough, and citations to scientific literature were provided. ${ }^{6}$ Nevertheless, debates are still on-going amongst scientists, policy makers, media and lay public about what should be offered, to whom, in what way, and if or how it is subject to governmental regulatory scrutiny. Together they aim to balance protection of customers, society and health care system as a whole on one side to the support of autonomy and innovation on the other side. ${ }^{78}$ However, some methodological, practical and ethical issues surrounding this concept remain.

\section{Issues regarding genetic testing at different stages}

Firstly, reliable pre-test information provision is currently limited, thereby impairing informed decision-making on DTC-GT by the general public. In western populations, the majority of people is currently still unaware of DTC-GT ${ }^{9-12}$ and even more are inadequately informed of what the concept entails. Most will therefore not yet have formed an opinion on genetic testing for disease risks. This means that it is an important and relevant moment to provide the general public with reliable information on the topic.

Secondly, disease risks are not solely determined by genetic factors, but are the result of an accumulation and interaction of multiple types of risk factors, including genetic but also lifestyle, environmental, and occupational factors. Integration of all these types of risk factors into the prediction models is needed for more accurate and relevant disease risk prediction.

Thirdly, learning of disease risks does not (necessarily) lead to behaviour change. Previous studies have been showing limited effects with regard to behaviour change. In addition, if behaviour change is achieved, long term maintenance is often low or absent. ${ }^{13-17}$ Furthermore, worries have been expressed that it may even lead to negative effects, including adverse psychological reactions as well as unnecessarily burdening the health care system by medical follow-up. ${ }^{18,19}$ In order to contribute to the discussion on the risks and benefits balance, it is necessary to get more insight into the impacts.

In line with this, risk reports currently focus mostly on conveying genetic disease risk information without translating this into management options. As a result, it has been argued that actionability with regard to disease prevention is low. So if our goal is 
to achieve behaviour change, in order to improve health or prevent deterioration on the long term, concrete advice to reduce disease risk would be helpful. Furthermore, additional support may be necessary if we want to achieve (long-term) behaviour change. Assistance from a HCP may be required for individuals to convert their risk reports and concrete lifestyle advice into actual behaviour change and maintenance.

\section{A possible step forward: Health Potential}

To address these issues, we propose a new disease prevention service: Health Potential (www.healthpotential.eu). Health Potential is a personal informational service on $20+$ common chronic diseases, providing its clients with the most up-to-date and comprehensive disease risk estimates, including personalised lifestyle advice.

It includes the following attributes:

Improved disease risk estimation. Health Potential combines information on genetic, lifestyle and environmental factors to get to a more comprehensive estimation of disease risk.

Highest levels of evidence. Disease risk estimates are derived from meta-analyses and meta-meta-analyses only to obtain estimates at the highest level of evidence. Scientific literature is searched every 6 months for new research on associations between risk factors and each disease at a meta-analytic level, which is then used to update the evidence to the latest scientific insights.

Involvement of a coach to improve understanding of the results. Each client is supervised by a health coach, which is trained to understand, translate and deliver the Health Potential results to their client.

Personalised and concrete behaviour change advice. The result report includes actionable advice for each disease, which can easily be implemented by clients to reduce their disease risk.

Involvement of a coach to stimulate behaviour change and maintenance. The coach has a relevant health-related background and is experienced in guiding clients in behaviour change. Using the Health Potential report as a basis, they can explore and implement strategies for behaviour change and maintenance together, using his professional network and resources to establish swift transitions to appropriate help and support 
Coaching, not gatekeeping. The Health Potential coach functions as an informant and facilitator, and does not take on the role of a gatekeeper, as would be the case when a physician decides whether or not a client gets a specific test. This way, the 'direct-toconsumer character' of everyone being able to obtain the test is maintained, while at the same time offering the assistance and support of involving a HCP.

The primary aim of the Health Potential service is to induce behaviour change, while limiting adverse effects such as misinterpretation, distress and unnecessary medical consumption.

\section{Aims of this thesis}

The overall aim of this thesis is to examine the aforementioned deficits of the current DTC-GT approach, and to propose Health Potential as an alternative concept to address these deficits.

\section{Thesis outline}

Chapter 2 starts at the pre-testing phase, where we studied characteristics of the expected consumer population of DTC-GT in order to provide assistance in the development of pre-test information to enhance informed decision-making. This chapter presents the results of an original study on factors associated with acceptability and consideration of undergoing DTC-GT in the Netherlands. The foundation for integration of lifestyle, environmental and occupational risk factors in disease risk prediction will be laid in chapter 3, using COPD as an example. In chapter 4 the effects of DTC-GT with regard to behaviour change, psychological effects and post-test medical consumption are studied in a systematic review with meta-analyses. The focus of chapter $\mathbf{5}$ on the other hand is to provide the foundation for improving actionability of results through offering concrete lifestyle advice. This is done by studying modifiable risk factors of disease using bladder cancer as an example. The study protocol for the Health Potential trial, in which the ability of the Health Potential service to change behaviour is studied, is presented in chapter 6. Finally, chapter 7 contains the general discussion. 


\section{References}

1. World Health Organization. Projections of mortality and causes of death, 2015 and 2030.

2. World Health Organization. World Health Statistics 2017: Monitoring health for the SDGs. 2017.

3. World Health Organization. Global Health Risks-Mortality and burden of disease attributable to selected major risks. Cancer. 2017.

4. Offit K. Personalized medicine: new genomics, old lessons. Human genetics. 2011; 130: 3-14.

5. Fung B. Bowing again to the FDA, 23andMe stops issuing health-related genetic reports. The Washington Post. 2016.

6. FDA. Press Announcements - FDA allows marketing of first direct-to-consumer tests that provide genetic risk information for certain conditions. 2017.

7. Rafiq M, Ianuale C, Ricciardi W and Boccia S. Direct-to-consumer genetic testing: A systematic review of european guidelines, recommendations, and position statements. Genetic testing and molecular biomarkers. 2015; 19: 535-47.

8. Vayena E. Direct-to-consumer genomics on the scales of autonomy. Journal of medical ethics. 2015; 41: 310-4.

9. Cherkas LF, Harris JM, Levinson E, Spector TD and Prainsack B. A survey of UK public interest in internet-based personal genome testing. PloS one. 2010; 5: e13473.

10. Ortiz AP, Lopez M, Flores LT, et al. Awareness of direct-to-consumer genetic tests and use of genetic tests among Puerto Rican adults, 2009. Preventing chronic disease. 2011; 8: A110.

11. Agurs-Collins T, Ferrer R, Ottenbacher A, Waters EA, O'Connell ME and Hamilton JG. Public Awareness of Direct-to-Consumer Genetic Tests: Findings from the 2013 US Health Information National Trends Survey. Journal of Cancer Education. 2015; 30: 799-807.

12. Mavroidopoulou V, Xera E and Mollaki V. Awareness, attitudes and perspectives of direct-toconsumer genetic testing in Greece: a survey of potential consumers. Journal of human genetics. 2015; 60: 515-23.

13. Smerecnik C, Grispen JE and Quaak M. Effectiveness of testing for genetic susceptibility to smoking-related diseases on smoking cessation outcomes: a systematic review and meta-analysis. Tobacco control. 2012: tc. 2011.042739.

14. Bloss CS, Wineinger NE, Darst BF, Schork NJ and Topol EJ. Impact of direct-to-consumer genomic testing at long term follow-up. Journal of medical genetics. 2013; 50: 393-400.

15. Bloss CS, Schork NJ and Topol EJ. Effect of direct-to-consumer genomewide profiling to assess disease risk. The New England journal of medicine. 2011; 364: 524-34.

16. Egglestone C, Morris A and O'Brien A. Effect of direct-to-consumer genetic tests on health behaviour and anxiety: a survey of consumers and potential consumers. Journal of genetic counseling. 2013; 22: 565-75.

17. Olfson E, Hartz S, Carere DA, et al. Implications of personal genomic testing for health behaviors: the case of smoking. Nicotine \& Tobacco Research. 2016; 18: 2273-7.

18. McGuire AL and Burke W. An unwelcome side effect of direct-to-consumer personal genome testing: raiding the medical commons. Jama. 2008; 300: 2669-71.

19. Annes JP, Giovanni MA and Murray MF. Risks of presymptomatic direct-to-consumer genetic testing. The New England journal of medicine. 2010; 363: 1100-1. 
Chapter 1| General introduction 


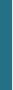




\section{Abstract}

Background: With interest in personalised health care growing, so is interest in personal genetic testing. This is now offered direct-to-consumer, thereby referred to as direct-to-consumer genetic testing (DTC-GT). Criticisms have been expressed on whether a truly informed decision to undergo testing is attained in these services. In order to provide relevant information to achieve this, knowing the characteristics of the expected user population is helpful. Therefore, the aim of this study is to identify characteristics of individuals who (1) find the concept of DTC-GT acceptable, and (2) consider undergoing DTC-GT in the distant or near future.

Methods: This cross-sectional study investigated factors associated with acceptability, consideration and intention in the Dutch general population. Studied variables included awareness, principles and how-to knowledge, attitude, innovativeness, and multiple demographic characteristics. Generalised linear models were applied to identify associated variables.

Results: Full data was obtained for 836 respondents. Of those, 18.3\% found DTCGT somewhat or totally acceptable, whereas $12.6 \%$ considered and $5.5 \%$ intended to undergo DTC-GT in the distant or near future. Acceptability was greater with lower principles knowledge, and consideration and intention with lower how-to knowledge. A more positive attitude and greater innovativeness were associated with an increase in all three outcomes.

Conclusion: Informed decision-making may be hampered as individuals with lower how-to knowledge were found to be more interested in pursuing testing. The identified characteristics can be used in development and distribution of public and personalized information, in order to help consumers make a truly informed decision. 


\section{Introduction}

Personalised health care, i.e. health care tailored to the individual's characteristics and needs, is undergoing rapid developments. This is, at least partially, the result of increased pressure from authorities of taking responsibility for one's own health. In this light, an interesting and emerging scientific field is that of personal genetic disease-risk testing, which aims to estimate risks of developing specific common chronic diseases in the future, based on genetic profiling. In contrast to the traditional genetic testing in the medical setting, which is often of a diagnostic nature, personal genetic disease-risk testing is mostly non-diagnostic but of preventive nature, and aimed directly at the consumer without interference of a health care professional. Thereby it is often referred to as direct-to-consumer genetic testing (DTC-GT). Such services are mostly offered online; consumers send their DNA sample by mail and receive their results in an online report. $^{1}$

One of the criticisms of DTC-GT is the dubiousness of attaining a truly informed decision of the customer to undergo testing. Indeed, companies have now mostly offered their product as a non-medical product intended 'for informational purposes only', thereby deferring attaining a true informed consent to a mere agreement to terms of purchase, such as agreeing to use and store the consumer's samples and data. ${ }^{2,3}$ Yet, a majority of companies simultaneously and conflictingly state on their websites that results can be used to inform health decisions. ${ }^{4}$ Therefore, considering the nature of the information from such genetic tests, consent should not be reduced to merely ticking a box but instead companies should strive to attain a well-informed decision. ${ }^{5}$

An informed decision should, at least, be (1) based on sufficient relevant information and (2) consistent with the values of the individual making the decision. ${ }^{6}$ This means that, primarily, complete and objective information provision is essential. However, although DTC-GT companies should feel morally obliged to provide this information, the majority of their websites were found to be misleading, overemphasising benefits and requiring high literacy. ${ }^{4,7,8}$ Therefore, other stakeholders such as governmental authorities or the medical community should step in to ensure adequate pre-test education, amongst others through availability of high-quality, complete and objective information. In order to develop and distribute this information more effectively, it is helpful to know the characteristics of the expected user population. Therefore, the aim of this study is to identify characteristics of individuals who (1) find the concept of DTC-GT acceptable, (2) consider undergoing DTC-GT at some point future, or (3) intend to undergo DTC-GT in the coming year. 


\section{Methods}

\section{Design}

This cross-sectional analysis is part of a study into knowledge and attitude towards DTC-GT, and determinants of intended use in the Netherlands. It is based on an online survey, which was distributed in June 2017. The survey was anonymous, confidential and voluntary. The study protocol was approved by the Medical Ethics Review Committee of Zuyderland (number 17-N-58).

\section{Participants}

Participants were recruited from an online panel (Flycatcher, Maastricht, The Netherlands) representative of the Dutch adult (18+) population, based on age, gender and education level. Panel members were approached via e-mail and were required to sign an electronic informed consent form before participating. They received a personalised link to the web questionnaire. A reminder e-mail was sent after five days in case of non-response.

\section{Data collection}

Due to the presumed relative unawareness of DTC-GT among the Dutch population, the survey started with a short description of the concept, particularly focussing on disease-related and lifestyle-related DTC-GT (Supplementary text 1 ). The results of the current report focuses solely on two types of disease-related testing: (1) genetic testing to estimate disease risks for future development of common chronic diseases, and (2) carrier status testing, which tests whether an individual carries a single copy of a genetic mutation which will cause a genetic disorder when present in both copies. This latter type of testing is relevant for (future) offspring, as it could result in disease if both parents each pass on one mutated copy.

The items included in the questionnaire were composed based on existing models of behaviour change ${ }^{9,10}$, previous literature ${ }^{11-17}$ and expert opinion in the field of genetics, DTC-GT, health education and informed decision-making.

The following quantitative items were collected through the survey:

\section{Outcome parameters}

Acceptability of the DTC approach in genetic testing was measured as "How acceptable is it in your opinion that private companies provide results of genetic tests directly to consumer without mandatory involvement of a health professional (doctor, geneticist, counsellor)?” Respondents had to rate their answer on a 5-point scale (1 - Completely unacceptable to 5 - Completely acceptable). 
Consideration referred to a general intent to undergo DTC-GT for disease-related purposes at some time in the future, reflecting a willingness to think about undergoing testing but not yet intending to do so. Consideration was measured using the following question: "Would you consider doing DTC genetic testing for disease related purposes in the future?” This was rated on a 5-point scale (1 - Certainly no to 5 - Certainly yes).

Intention to undergo testing was operationalized as the intention to undergo testing in the next year, reflecting a more committing intent compared to consideration: ("Do you intend to use a DTC genetic test for disease related purposes in the next year?" Both were rated on a 5-point scale (1 - Certainly no to 5 - Certainly yes).

\section{Independent variables}

Awareness of DTC-GT for disease-related purposes was asked with one question per purpose: "Prior to participating in this research, had you ever heard of DTC genetic tests for disease-related purposes?” This resulted in a yes/no answer.

Previous use of DTC-GT for disease-related purposes was measured by first asking whether the participant had ever undergone genetic testing to receive any type of information on their personal genetic profile. Secondly, if yes, whether this genetic test was obtained direct-to-consumer, through a medical professional or otherwise. Thirdly, the purpose of this test was asked through multiple-choice options, including the answer options of disease-related purposes. This resulted in a yes/no answer.

Two types of knowledge were distinguished: principles knowledge and how-to knowledge. Principles knowledge, referring to the theoretical information about the functioning principles of an innovation (i.e. DTC-GT), refers in our context to the understanding of principles of genetics, which was measured with 14 true/false questions, with the added option of 'don't know' (Supplementary text 2). Answers were rated as correct or incorrect (with 'don't know' considered incorrect) and a total sum of correct answers was calculated. How-to knowledge, originally described as the practical knowledge required to use an innovation properly, refers in our context to the understanding of the DTC-GT results. Sample results based on reports from existing companies were given, reflecting two types of information: on carrier status and on risk of disease (Supplementary text 3). Understanding of these results was evaluated with six multiple-choice or open questions, and rated as correct or incorrect to come to a total sum of correct answers. (Supplementary text 4)

Attitude towards DTC-GT for disease-related purposes was measured using eight items of opposite meaning, which was rated on a semantic differential 5-point scale. Respondents 
were asked: "Direct-to-consumer genetic testing for disease-related purposes is...", with the following answer items: beneficial to the health of the user - harmful to the health of the user, beneficial for society - harmful for society, leading to healthier lifestyle of its users - leading to unhealthier lifestyle of its users, leading to increased feelings of privacy of the user - leading to decreased feelings of privacy of the user, ethical - unethical, useful - not useful, reassuring - frightening, important - not important. These items were based on previously reported attributes towards DTC-GT ${ }^{18-23}$ combined with expert opinion. Negative items were reverse scored so that a higher score represents a more positive attitude. Reliability of the resulting scale was high at $\alpha=0.85$.

Innovativeness is an individual's value towards innovation (i.e. DTC-GT for diseaserelated purposes), reflecting the relative earliness of adopting new ideas compared to others. ${ }^{9}$ In our study, this was operationalized as how open the participant is in terms of using new tests or treatments in the medical domain. For this a medical domain-specific innovativeness scale was used. ${ }^{24,25}$ Participants were asked to rate three statements ( 1 , "If I heard of a new medical test or treatment, I would try to find out more about it"; 2, "Among my friends, I am usually one of the first to find out about a new medical test or treatment"; and 3 (reverse-scored), "In general, I am hesitant to undergo a new medical test or treatment,") on a 5-point Likert scale from strongly disagree to strongly agree. Higher scores reflect a greater level of innovativeness. Reliability was satisfactory at $\alpha=0.69$.

Finally, the following variables were collected: age, gender, educational level, being religious (yes/no), having a partner (yes/no), having biological children (yes/no), having adopted or step children (yes/no), planning to have children (yes/maybe/don't know/ no), having family members diagnosed with a genetic disease (yes/no/don't know), having a chronic disease diagnosed by a physician (yes/no), and self-rated health (1Poor to 5-Excellent).

\section{Data analysis}

To identify factors associated with acceptability, consideration and intention of DTCGT we applied a generalized linear model. First, we ran univariate analyses with a $\alpha=$ 0.20 cut-off to identify the individual factors to be entered into the multivariate model. We checked linearity for each variable that originated from a Likert scale question. If the association appeared to be non-linear, the variable was then entered into the model as categorical variables. In the final model, a $\alpha=0.05$ cut-off was used to finally determine the set of associated variables. All analyses were done in Stata v14 (StataCorp, 2015). 


\section{Results}

\section{Study population}

Of the 1693 panellists who were contacted, 1063 responded to the questionnaire (63\% response rate). Of these, nine were excluded due to low response quality (e.g. repeated patterns of answers) and 218 had to be excluded due to missing answers, leaving 836 respondents with complete data to be included in our analyses.

The main characteristics of the study sample are displayed in Table 1 . The population was comparable to the Dutch population with regard to gender, age and education. The middle education level group was overrepresented, with $43.3 \%$ compared to $32.5 \%$ and $24.2 \%$ for low and high level respectively, but not different from distribution in the Dutch population. Most respondents had a partner and were non-religious. The great majority $(76.6 \%)$ did not plan to have children, $62.0 \%$ had biological children and $6.8 \%$ had adopted or step children. Most respondents (51.6\%) rated their health as 'good'. Approximately a third of respondents had a chronic disease and $22.7 \%$ had a family member with a genetic disease. Compared to non-respondents, respondents did not differ by gender or education, but a greater proportion of non-responders was seen in the youngest category and a smaller proportion in the older category $\left(\mathrm{X}^{2}(2)=15.78\right.$; $\mathrm{p}<0.001)$.

\section{Correlates of acceptability, consideration and intention}

Nearly a fifth of respondents (18.3\%) found DTC-GT somewhat or totally acceptable. Up to $12.6 \%$ probably or definitely considered undergoing DTC-GT in the distant future, whereas $5.5 \%$ intended to undergo DTC-GT in the coming year. Details on all answer categories are displayed in Table 2 .

\section{Acceptability}

Respondents were more acceptable of DTC-GT when they had lower levels of principles knowledge. Also, a more positive attitude and greater innovativeness were associated with greater acceptability. In addition, younger and non-religious people, as well as those with poor or excellent self-rated health tended to find DTC-GT more acceptable. (Table 3 and supplementary table 1)

\section{Consideration and intention}

Correlates of consideration and intention were largely overlapping. Firstly, respondents with lower levels of how-to knowledge were more likely to have greater consideration and intention to undergo DTC-GT. In addition, a more positive attitude and greater innovativeness were also associated with greater consideration and intention. Finally, 
Chapter 2 | Acceptability, consideration and intention of DTC-GT uptake

Table 1 Descriptive statistics of the study sample

\begin{tabular}{|c|c|c|c|c|}
\hline & & $\mathbf{n}$ & $\%$ & $\begin{array}{l}\text { Dutch general } \\
\text { population } \%^{\mathrm{ab}}\end{array}$ \\
\hline Total & & 836 & & \\
\hline \multirow[t]{3}{*}{ Age } & $18-39$ & 250 & 29.9 & 35 \\
\hline & $40-59$ & 299 & 35.8 & 38 \\
\hline & $60+$ & 287 & 34.3 & 30 \\
\hline \multirow[t]{2}{*}{ Gender } & Male & 422 & 50.5 & 49 \\
\hline & Female & 414 & 49.5 & 51 \\
\hline \multirow[t]{3}{*}{ Education level } & Low & 272 & 32.5 & 31 \\
\hline & Middle & 362 & 43.3 & 44 \\
\hline & High & 202 & 24.2 & 25 \\
\hline \multirow[t]{2}{*}{ Partner } & Yes & 600 & 71.8 & \\
\hline & No & 236 & 28.2 & \\
\hline \multirow[t]{2}{*}{ Religious } & Yes & 357 & 42.7 & \\
\hline & No & 479 & 57.3 & \\
\hline \multirow[t]{4}{*}{ Planning to have children } & Yes & 129 & 15.4 & \\
\hline & Maybe & 29 & 3.5 & \\
\hline & No & 640 & 76.6 & \\
\hline & Don't know & 38 & 4.5 & \\
\hline \multirow[t]{2}{*}{ Biological children } & Yes & 518 & 62.0 & \\
\hline & No & 318 & 38.0 & \\
\hline \multirow[t]{2}{*}{ Adopted or stepchildren } & Yes & 57 & 6.8 & \\
\hline & No & 779 & 93.2 & \\
\hline \multirow[t]{5}{*}{ Self-rated health } & Excellent & 46 & 5.5 & \\
\hline & Very good & 144 & 17.2 & \\
\hline & Good & 431 & 51.6 & \\
\hline & Fair & 187 & 22.4 & \\
\hline & Poor & 28 & 3.3 & \\
\hline \multirow[t]{2}{*}{ Having a chronic disease } & Yes & 303 & 36.2 & \\
\hline & No & 533 & 63.8 & \\
\hline \multirow[t]{3}{*}{ Genetic disease in the family } & Yes & 190 & 22.7 & \\
\hline & No & 450 & 53.8 & \\
\hline & Don't know & 196 & 23.4 & \\
\hline \multirow{2}{*}{$\begin{array}{l}\text { Previous use of DTC-GT for } \\
\text { disease-related purposes }\end{array}$} & Yes & 2 & 0.2 & \\
\hline & No & 0 & 99.8 & \\
\hline \multirow{2}{*}{$\begin{array}{l}\text { Awareness of DTC-GT for } \\
\text { disease-related purposes }\end{array}$} & Yes & 238 & 28.5 & \\
\hline & No & 598 & 71.5 & \\
\hline
\end{tabular}

Legend: a Gouden Standaard - CBS in collaboration with MOA 2016; b Percentages may not add up to $100 \%$ due to rounding 
respondents were more likely to consider or intend to undergoing DTC-GT when they were younger, had a better rating of health. In contrast, having a genetic disease in the family was associated with greater consideration but not intention. (Table 3 and supplementary table 1)

Table 2 Summary statistics for outcome parameters

\begin{tabular}{lll}
\hline Acceptability & $\mathbf{n}$ & $\mathbf{\%}$ \\
\hline Totally unacceptable & 229 & 27.4 \\
Somewhat unacceptable & 243 & 29.1 \\
Neutral & 211 & 25.2 \\
Somewhat acceptable & 101 & 12.1 \\
Totally acceptable & 52 & 6.2 \\
\hline Consideration & $\mathbf{n}$ & $\mathbf{\%}$ \\
\hline Definitely not & 242 & 28.9 \\
Probably not & 214 & 25.6 \\
Maybe / Maybe not & 275 & 32.9 \\
Probably yes & 81 & 9.7 \\
Definitely yes & 24 & 2.9 \\
\hline Intention & $\mathbf{n}$ & $\mathbf{\%}$ \\
\hline Definitely not & 438 & 52.4 \\
Probably not & 210 & 25.1 \\
Maybe / Maybe not & 142 & 17.0 \\
Probably yes & 30 & 3.6 \\
Definitely yes & 16 & 1.9 \\
\hline
\end{tabular}

Legend: $\mathrm{n}=$ Number of respondents

\section{Discussion}

The aim of this paper was to identify characteristics related to acceptability, consideration and intention of DTC-GT.

\section{Innovativeness}

Greater innovativeness lies at the basis of early adoption of an innovation. ${ }^{9}$ As expected, with DTC-GT being relatively new, our results show that greater innovativeness (as measured within the medical domain) was associated with greater acceptability, consideration and intention. One previous study on clinical genetic testing for BRCA1/2 also found that greater innovativeness was associated with awareness and uptake of testing. ${ }^{24}$ To date, this is the first study to examine innovativeness with regard to DTCGT and results indicate it as an important factor in predicting interest in DTC-GT. 
Chapter 2 | Acceptability, consideration and intention of DTC-GT uptake

Table 3 Multivariate analyses for acceptability, consideration and intention

\begin{tabular}{|c|c|c|c|c|}
\hline Acceptability & & $\mathbf{b}$ & SE b & p-value \\
\hline Age & Per 1 year increase & -0.015 & 0.003 & $0.000^{*}$ \\
\hline \multirow[t]{3}{*}{ Education } & Low & Ref & & \\
\hline & Medium & 0.111 & 0.092 & 0.227 \\
\hline & High & 0.040 & 0.110 & 0.719 \\
\hline \multirow[t]{2}{*}{ Being religious } & Yes & -0.160 & 0.076 & $0.035^{*}$ \\
\hline & No & Ref & & \\
\hline \multirow[t]{4}{*}{ Planning to have children } & Yes & -0.269 & 0.142 & 0.057 \\
\hline & Maybe & -0.013 & 0.219 & 0.951 \\
\hline & Don’t know & 0.069 & 0.198 & 0.727 \\
\hline & No & Ref & & \\
\hline \multirow[t]{2}{*}{ Having biological children } & Yes & 0.024 & 0.094 & 0.800 \\
\hline & No & Ref & & \\
\hline \multirow[t]{5}{*}{ Self-rated health } & 1 & 0.340 & 0.169 & $0.043^{*}$ \\
\hline & 2 & 0.066 & 0.107 & 0.539 \\
\hline & 3 & Ref & & \\
\hline & 4 & 0.100 & 0.097 & 0.303 \\
\hline & 5 & 0.627 & 0.211 & $0.003^{*}$ \\
\hline Principles knowledge & Per 1 increase in score & -0.048 & 0.014 & $0.001^{*}$ \\
\hline Attitude & Per 1 increase in score & 0.580 & 0.061 & $0.000^{*}$ \\
\hline Innovativeness & Per 1 increase in score & 0.175 & 0.044 & $0.000^{*}$ \\
\hline Consideration & & $\mathbf{b}$ & SE b & p-value \\
\hline Age & Per 1 year increase & -0.015 & 0.003 & $0.000^{*}$ \\
\hline \multirow[t]{3}{*}{ Education } & Low & Ref & & \\
\hline & Medium & 0.021 & 0.071 & 0.765 \\
\hline & High & 0.049 & 0.084 & 0.559 \\
\hline \multirow[t]{4}{*}{ Planning to have children } & Yes & -0.189 & 0.110 & 0.086 \\
\hline & Maybe & -0.146 & 0.169 & 0.390 \\
\hline & Don't know & -0.030 & 0.153 & 0.846 \\
\hline & No & Ref & & \\
\hline \multirow[t]{2}{*}{ Having biological children } & Yes & -0.045 & 0.075 & 0.529 \\
\hline & No & Ref & & \\
\hline \multirow{3}{*}{$\begin{array}{l}\text { Having a genetic disease } \\
\text { in the family }\end{array}$} & Yes & 0.231 & 0.075 & $0.002^{*}$ \\
\hline & Don't know & 0.037 & 0.073 & 0.613 \\
\hline & No & Ref & & \\
\hline \multirow[t]{2}{*}{ Having a chronic disease } & Yes & -0.051 & 0.071 & 0.470 \\
\hline & No & Ref & & \\
\hline Self-rated health & Per 1 increase in score & 0.081 & 0.037 & $0.041^{*}$ \\
\hline How-to knowledge & Per 1 increase in score & -0.052 & 0.020 & $0.010^{*}$ \\
\hline Attitude & Per 1 increase in score & 0.651 & 0.047 & $0.000^{*}$ \\
\hline Innovativeness & Per 1 increase in score & 0.410 & 0.034 & $0.000^{*}$ \\
\hline
\end{tabular}


Chapter 2 | Acceptability, consideration and intention of DTC-GT uptake

\begin{tabular}{lllll}
\hline Intention & & b & SE b & p-value \\
\hline Age & Per 1 year increase & -0.009 & 0.002 & $0.000^{*}$ \\
Education & Low & Ref & & \\
& Medium & 0.040 & 0.074 & 0.584 \\
& High & -0.018 & 0.087 & 0.837 \\
Having biological children & Yes & -0.010 & 0.073 & 0.886 \\
& No & Ref & & \\
Having a genetic disease & Yes & 0.090 & 0.077 & 0.243 \\
in the family & & & & \\
& Don't know & -0.017 & 0.076 & 0.827 \\
Self-rated health & No & Ref & & \\
How-to knowledge & Per 1 increase in score & 0.088 & 0.037 & $0.018^{*}$ \\
Attitude & Per 1 increase in score & -0.085 & 0.021 & $0.000^{*}$ \\
Innovativeness & Per 1 increase in score & 0.284 & 0.049 & $0.000^{*}$ \\
\hline
\end{tabular}

Legend: ${ }^{*} \alpha<0.05$

\section{Knowledge and attitudes}

Clearly, a first requirement for adoption to take place is awareness of the innovation. Nevertheless, our results showed no association between prior awareness of DTC-GT and acceptability, consideration or intention. On the other hand, our results showed that acceptability was greater with lower knowledge of principles of genetics (i.e. principles knowledge). Similarly, consideration and intention were greater with lower understanding of genetic test results (i.e. how-to knowledge). This is in line with a previous study among health-care professionals which found that non-geneticist HCPs, who may be assumed to have lower knowledge of genetics and genetic testing, were more positive about the benefits of genetic testing. ${ }^{26}$ Somewhat related, another study found that participants who had been exposed to information on risks of DTC BRCA testing had lower intentions of undergoing testing. ${ }^{27}$ Our findings can be interpreted as worrisome, as they may indicate that those individuals with the least understanding of genetic test results would be most interested in pursuing testing. This could also imply that the first prerequisite for informed decision-making, namely that it is based on sufficient relevant information, may often be violated. In addition, it may imply that with improved information provision to the general public, consideration might be lower.

Finally, attitude towards DTC-GT was positively associated with acceptability, consideration and intention, a finding that is consistent with other research. ${ }^{28}$ Coming back to informed decision-making, this could indicate that the decision to consider undergoing testing would meet the second prerequisite, namely that it is in line with one's personal values. 


\section{Demographic factors}

In addition, several demographic factors were found to be associated with acceptability, consideration and intention. Firstly, younger age was associated with all three outcomes, which is in line with other research. ${ }^{29,30}$ This may be explained by the greater impact testing may still have on future life, and younger individuals may have a greater general interest in innovation, for example in the embrace of new technology. Secondly, self-rated health was associated with acceptability, consideration and intention. For acceptability the association was U-shaped, with increased acceptability for both poor and excellent health. Consideration and intention both increased with more positive self-rated health. In addition, having a genetic disease in the family increased consideration. These findings may both be explained through different perceived utility. Namely, it was found that actual DTC-GT users with poorer self-reported health ${ }^{8}$ and members of the general population with serious disease in the family ${ }^{20}$ perceived results as having lower utility. Thirdly, identifying with a religion resulted in lower acceptability of DTC-GT. This is consistent with other research on genetic testing, which generally showed lower interest in genetic testing among people who identified with a religion. ${ }^{20}$ In contrast to previous research, we found no association with education ${ }^{11,31}$ or gender $^{8,11,30}$.

\section{Methodological strengths and limitations}

Our study has identified both new and previously identified factors associated with acceptability of and interest in undergoing DTC-GT. This was done in a large and representative sample of the Dutch population, thereby allowing generalisation of the results. Nevertheless, as with all research, findings should be critically evaluated. Firstly, where possible, validated or previously used questionnaires were used. However, due to the relative novelty of the topic, the questionnaire included several self-developed items. To improve validity as much as possible, existing questionnaires were modified to fit our research and experts were consulted. In addition, the questionnaire was successfully pretested in a small sample. Secondly, many of our respondents had not yet heard of DTC-GT prior to the survey. At the start of the survey, we offered an introduction to the topic (Supplementary text 1), which we aimed to be as objective and informative as possible. However, they had to base their opinion on the topic on this relatively brief description and do so in the relatively short amount of time they took to read it. Although there was no time limit to read and reflect on this introductory text, it is likely individuals read this without thorough pondering. As a result, decisions or answers are likely to reflect rather intuitive responses. Therefore, it may be relevant for a future study to attempt to replicate our results after informing respondents more elaborately, for example by offering a detailed description of obtained information and risks and benefits of testing, in order to obtain results from a well-informed decision. Finally, in behavioural research a well-known phenomenon is that of the intention-behaviour 
gap, which refers to the discrepancy between the intentions people make and the actual behaviour they perform. As a result, the factors associated with consideration and intention that we identified may not necessarily reflect factors of actual uptake of DTCGT. We therefore recommend that future studies focus on identifying factors associated with actual uptake.

\section{Conclusion}

Our research has identified a number of correlates of DTC-GT acceptability and the consideration to take up DTC-GT in the distant and near future. Of particular interest are the findings that lower knowledge of genetic principles was associated with greater acceptability, and that lower understanding of DTC-GT results was associated with greater consideration and intention to undergo genetic testing. These findings plea for investment in public and personalized information on basic genetic principles and the content of disease-related genetic testing results. The characteristics identified in this study can be used in the development and distribution of public and personalized information and decision support with regard to DTC-GT, in order to help consumers make an informed decision on whether or not to undergo testing. In addition, the FDA already requires positive comprehension tests for DTC-GT to receive market approval. ${ }^{32}$ This should be expected of all DTC-GT companies, including those not under FDA jurisdiction.

\section{Funding}

This project was funded by the Maastricht University Interfaculty Program 'Eatwell'. The funder had no influence in the design or execution of the research. 


\section{References}

1 Saukko P: State of play in direct-to-consumer genetic testing for lifestyle-related diseases: market, marketing content, user experiences and regulation. The Proceedings of the Nutrition Society 2013;72:53-60.

2 Phillips AM: Reading the fine print when buying your genetic self online: direct-to-consumer genetic testing terms and conditions. New genetics and society 2017;36:273-295.

3 Allyse MA, Robinson DH, Ferber MJ, Sharp RR: Direct-to-Consumer Testing 2.0: Emerging Models of Direct-to-Consumer Genetic Testing. Mayo Clinic proceedings 2018;93:113-120.

4 Lachance CR, Erby LAH, Ford BM, Allen VC, Kaphingst KA: Informational content, literacy demands, and usability of websites offering health-related genetic tests directly to consumers. Genetics in Medicine 2010;12:304-312.

5 Borry P, Henneman L, Lakeman P, ten Kate LP, Cornel MC, Howard HC: Preconceptional genetic carrier testing and the commercial offer directly-to-consumers. Human Reproduction 2011;26:972-977.

6 van den Berg M, Timmermans DR, ten Kate LP, van Vugt JM, van der Wal G: Are pregnant women making informed choices about prenatal screening? Genetics in Medicine 2005;7:332.

7 Singleton A, Erby LH, Foisie KV, Kaphingst KA: Informed choice in direct-to-consumer genetic testing (DTCGT) websites: a content analysis of benefits, risks, and limitations. Journal of genetic counseling 2012;21:433-439.

8 Roberts JS, Gornick MC, Carere DA, Uhlmann WR, Ruffin MT, Green RC: Direct-to-Consumer Genetic Testing: User Motivations, Decision Making, and Perceived Utility of Results. Public health genomics 2017;20:36-45.

9 Rogers EM: Diffusion of innovations. Simon and Schuster, 2010.

10 Gomez P, Borges A, Pechmann C: Avoiding poor health or approaching good health: Does it matter? The conceptualization, measurement, and consequences of health regulatory focus. Journal of Consumer Psychology 2013;23:451-463.

11 Hall TO, Renz AD, Snapinn KW, Bowen DJ, Edwards KL: Awareness and uptake of direct-toconsumer genetic testing among cancer cases, their relatives, and controls: the Northwest Cancer Genetics Network. Genetic testing and molecular biomarkers 2012;16:744-748.

12 Carere DA, Kraft P, Kaphingst KA, Roberts JS, Green RC: Consumers report lower confidence in their genetics knowledge following direct-to-consumer personal genomic testing. Genetics in Medicine 2016;18:65-72.

13 Jallinoja P, Aro AR: Knowledge about genes and heredity among Finns. New genetics and society 1999;18:101-110.

14 Molster C, Charles T, Samanek A, O'Leary P: Australian study on public knowledge of human genetics and health. Public health genomics 2009;12:84-91.

15 Furr LA, Kelly SE: The Genetic Knowledge Index: developing a standard measure of genetic knowledge. Genetic testing 1999;3:193-199.

16 Bowling BV, Acra EE, Wang L, Myers MF, Dean GE, Markle GC, Moskalik CL, Huether CA: Development and evaluation of a genetics literacy assessment instrument for undergraduates. Genetics 2008;178:15-22.

17 Smerecnik C, Mesters I, De Vries N, De Vries H: Applying a theory-based framework to understand public knowledge of genetic risk factors: a case for the distinction between how-to knowledge and principles knowledge. Public health genomics 2011;14:259-270.

18 Paul NW, Banerjee M, Michl S: Captious certainties: makings, meanings and misreadings of consumer-oriented genetic testing. Journal of community genetics 2014;5:81-87.

19 Su P: Direct-to-consumer genetic testing: a comprehensive view. The Yale journal of biology and medicine 2013;86:359-365.

20 Mahlmann L, Rocke C, Brand A, Hafen E, Vayena E: Attitudes towards personal genomics among older Swiss adults: An exploratory study. Applied and Translational Genomics 2016;8:9-15. 
21 Quaak M, Smerecnik C, van Schooten FJ, de Vries H, van Schayck CP: Knowledge, attitudes and preferences regarding genetic testing for smoking cessation. A cross-sectional survey among Dutch smokers. BMJ open 2012;2:e000321.

22 Henneman L, Timmermans DR, Wal GVD: Public attitudes toward genetic testing: perceived benefits and objections. Genetic testing 2006;10:139-145.

23 Haga SB, Barry WT, Mills R, Ginsburg GS, Svetkey L, Sullivan J, Willard HF: Public knowledge of and attitudes toward genetics and genetic testing. Genetic testing and molecular biomarkers 2013; 17:327-335.

24 Armstrong K, Weiner J, Weber B, Asch DA: Early adoption of BRCA1/2 testing: who and why. Genetics in Medicine 2003;5:92.

25 Goldsmith RE, Hofacker CF: Measuring consumer innovativeness. Journal of the academy of marketing science 1991;19:209-221.

26 Goldsmith L, Jackson L, O'Connor A, Skirton H: Direct-to-consumer genomic testing from the perspective of the health professional: A systematic review of the literature. Journal of Community Genetics 2013;4:169-180.

27 Gray SW, Hornik RC, Schwartz JS, Armstrong K: The impact of risk information exposure on women's beliefs about direct-to-consumer genetic testing for BRCA mutations. Clinical genetics 2012;81:29-37.

28 Wade CH, Shiloh S, Woolford SW, Roberts JS, Alford SH, Marteau TM, Biesecker BB: Modelling decisions to undergo genetic testing for susceptibility to common health conditions: an ancillary study of the Multiplex Initiative. Psychology \& health 2012;27:430-444.

29 Mavroidopoulou V, Xera E, Mollaki V: Awareness, attitudes and perspectives of direct-to-consumer genetic testing in Greece: a survey of potential consumers. Journal of human genetics 2015;60:515523.

30 Cherkas LF, Harris JM, Levinson E, Spector TD, Prainsack B: A survey of UK public interest in internet-based personal genome testing. PloS one 2010;5:e13473.

31 Vermeulen E, Henneman L, van El CG, Cornel MC: Public attitudes towards preventive genomics and personal interest in genetic testing to prevent disease: a survey study. The European Journal of Public Health 2013;24:768-775.

32 US Food and Drug Administration: Evaluation of automatic class III designation for the 23andMe Personal Genome Service carrier screening test for Bloom syndrome. (Decision summary) 


\section{Supplementary material}

\section{Supplementary text 1 \\ Introductory text in survey on direct-to-consumer genetic testing \\ What are direct-to-consumer genetic tests?}

Nowadays, new DNA tests (genetic tests) are on the market. These tests assess a person's genetic profile for a variety of information. Companies or research institutes sell these tests directly to a consumer (hence the name direct-to-consumer), without involvement of a health professional. People can order these tests themselves online.

These tests are called direct-to-consumer (DTC) genetic tests.

The purposes of the testing can be very diverse. In this survey, we focus on two of them: (1) disease-related testing and (2) lifestyle-related testing.

(1) DTC genetic testing on disease-related topics provide information on your personal risk of developing diseases in the future, (such as cancers, heart failure, Alzheimer's, Type 2 diabetes, etc.). Additionally, these tests focus on identifying carrier status of a genetic mutation (a change in genetic information, the DNA), which means you have the mutation but you do not develop the disease yourself. This is relevant because you can pass on this mutation to your children.

(2) Direct-to-consumer genetic tests for lifestyle-related purposes provide information on your genetic predisposition for athletic ability. For example, based on your DNA, do you have more talent for endurance or sprinter type sports? On the other hand, these tests aim to predict how you respond to foods and beverages you consume. Those tests do not predict any diseases or health conditions. 


\section{Supplementary text 2}

Principles knowledge questions

1. Healthy parents can have a child with an inherited disease (True)

2. If your close relatives have diabetes or heart disease, you are more likely to develop these conditions (True)

3. Some genetic disorders occur more often within particular ethnic groups (True)

4. Most genetic disorders are caused by only a single gene (False)

5. Once a genetic marker for a disorder is identified in a person, the disorder can usually be prevented or cured (False)

6. A disease is only genetically determined if more than one family member is affected (False)

7. Some genetic disorders occur later in adult life (True)

8. A healthy lifestyle can prevent or lessen the negative consequences of having genetic predispositions to some diseases (True)

9. The environment has little or no effect on how genes contribute to disease (False)

10. One can see a gene with the naked eye. (False)

11. A gene is a molecule that controls hereditary characteristics. (True)

12. A gene is a piece of DNA. (True)

13. A gene is a part of a chromosome. (True)

14. Different body parts include different genes. (False) 


\section{Supplementary text 3}

How-to knowledge scenarios

\section{Scenario 1}

Imagine you have ordered a genetic test and received the results. The results are from a test assessing "carrier status", which means that you receive information whether you are carrying a variant of the gene for a genetic disease, which you could pass on to your children. Read the example results of this genetic test below and answer the questions to your best knowledge.

\section{Disease X Carrier Status Results}

You are a carrier. You could pass this variant on to your children.

We detected one variant for Disease X. People with only one variant are not expected to have Disease X.

Your results may be relevant if you are thinking about starting a family.

If you and your partner are both carriers, each child may have a $25 \%$ chance of having this condition.

\section{Scenario 2}

On the next page of the results, you receive the information about what your risk to get the Disease $Y$ is. Read the text and answer the questions below.

\section{Summary}

\begin{tabular}{|lll|}
\hline Disease & Lifetime risk & Current total risk \\
\hline Disease $\mathrm{Y}$ & $15 / 100$ & 4.47 \\
\hline
\end{tabular}




\section{Lifetime risks}

On average, 15/100 people of your age will be diagnosed with Disease Y during the rest of their lives. This is shown in the following figure: the orange men are people who will get the diagnosis of Disease $\mathrm{Y}$ during the rest of their lives.

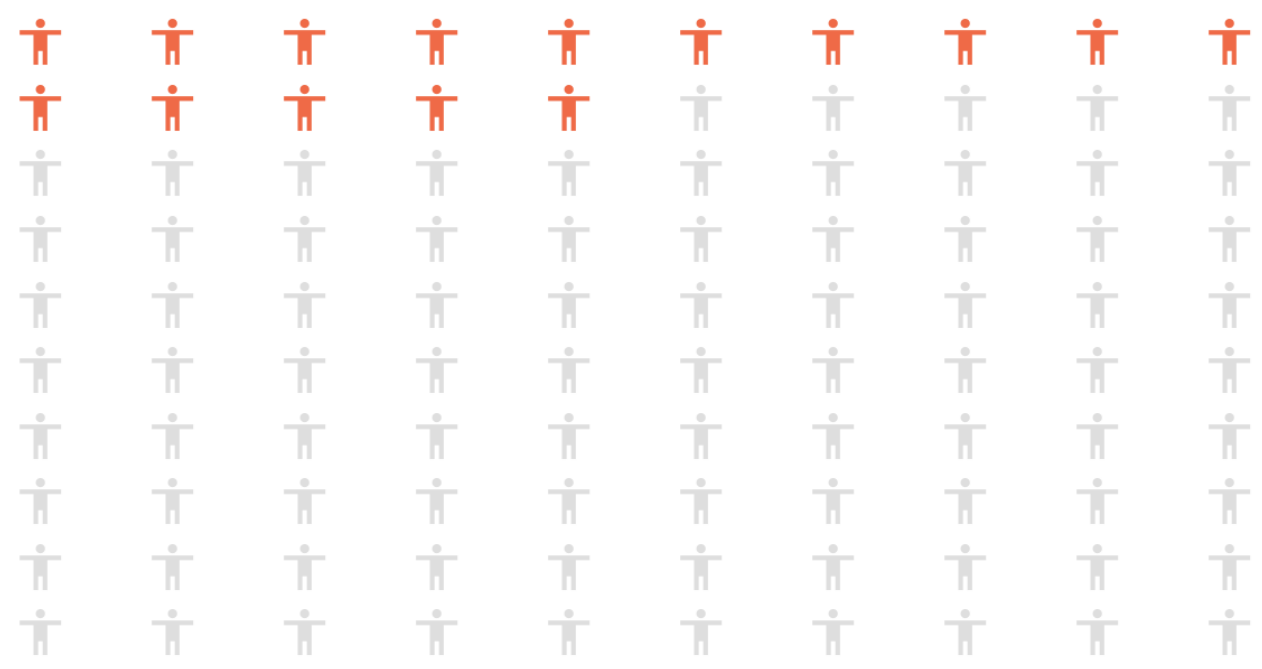

Personal risks

Total current risk: Based on your personal information, your risk of developing Disease $\mathrm{Y}$ is currently increased with $347 \%(\mathrm{RR}=4.47)$. With an optimal lifestyle you can bring it back to an increase of $81 \%(\mathrm{RR}=1.81)$. 


\section{Supplementary text 4}

How-to knowledge questions

\section{Scenario 1}

1. Based on the above results, what is your chance of displaying the symptoms of / (getting) the disease X? (correct answer: $\mathrm{d}$ )

a) I have a high-to medium chance

b) I have a medium-to-low chance

c) I have a zero chance

d) There is not enough information in the text to answer this question

e) I don't know

2. Based on above results, what is the chance (in \%) that your children will display symptoms of Disease $\mathrm{X}$ if your partner is also a carrier of Disease $\mathrm{X}$ and does not show symptoms of a disease (correct answer: $25 \%$ )

3. Based on above results, what is the chance (in \%) that your children will display symptoms of Disease X if your partner is not a carrier of the Disease X (correct answer: $0 \%)$

\section{Scenario 2}

1. Based on the results, what is your personal lifetime risk of getting the disease? (correct answer: e)
a) No risk
b) Low risk
c) Medium risk
d) High risk
e) Cannot determine from given data
f) I don't know

2. According to this information, what percentage of the people in the population will get this disease during their lifetime? (correct answer: 15\%) 
3. Is your current lifetime risk higher than average person lifetime risk for getting the disease? (correct answer: a)
a) Yes, it is higher
b) No, it is lower
c) No, it is equal
d) Cannot determine from given data
e) I don't know 
Chapter 2 | Acceptability, consideration and intention of DTC-GT uptake

\section{Supplementary table 1}

Supplementary table 1 Univariate analyses for acceptability, consideration and intention

\begin{tabular}{|c|c|c|c|c|c|}
\hline Acceptability & & $\mathbf{b}$ & SE b & p-value & $\begin{array}{l}\text { Non-linearity } \\
\text { test (p)\# }\end{array}$ \\
\hline \multirow[t]{2}{*}{ Gender } & Male & Ref & & & \\
\hline & Female & 0.084 & 0.083 & 0.304 & N/A \\
\hline Age in years & Per 1 year increase & -0.009 & 0.002 & $0.000^{*}$ & 0.203 \\
\hline \multirow[t]{3}{*}{ Education } & Low & Ref & & & \\
\hline & Medium & 0.050 & 0.095 & 0.602 & N/A \\
\hline & High & -0.155 & 0.110 & $0.158 \$$ & N/A \\
\hline \multirow{2}{*}{ Having a partner } & Yes & -0.024 & 0.091 & 0.795 & N/A \\
\hline & No & Ref & & & \\
\hline \multirow[t]{2}{*}{ Being religious } & Yes & -0.167 & 0.083 & $0.043^{*}$ & N/A \\
\hline & No & Ref & & & \\
\hline \multirow[t]{4}{*}{ Planning to have children } & Yes & 0.138 & 0.114 & 0.226 & N/A \\
\hline & Maybe & 0.228 & 0.225 & 0.309 & N/A \\
\hline & Don't know & 0.432 & 0.198 & $0.029^{*}$ & N/A \\
\hline & No & Ref & & & \\
\hline \multirow[t]{2}{*}{ Having biological children } & Yes & -0.125 & 0.084 & $0.138 \$$ & N/A \\
\hline & No & Ref & & & \\
\hline \multirow{2}{*}{$\begin{array}{l}\text { Having adopted or step } \\
\text { children }\end{array}$} & Yes & -0.003 & 0.163 & 0.983 & N/A \\
\hline & No & Ref & & & \\
\hline \multirow[t]{3}{*}{ Genetic disease in the family } & Yes & 0.079 & 0.103 & 0.440 & N/A \\
\hline & Don't know & 0.065 & 0.102 & 0.519 & N/A \\
\hline & No & Ref & & & \\
\hline \multirow[t]{2}{*}{ Having a chronic disease } & Yes & 0.066 & 0.085 & 0.438 & N/A \\
\hline & No & Ref & & & \\
\hline Self-rated health & $\begin{array}{l}\text { Per } 1 \text { point increase } \\
\text { in score }\end{array}$ & 0.032 & 0.047 & 0.497 & $0.013^{*}$ \\
\hline \multirow[t]{5}{*}{ Self-rated health } & 1 & 0.351 & 0.183 & $0.055 \$$ & \\
\hline & 2 & 0.066 & 0.114 & 0.559 & N/A \\
\hline & 3 & Ref & & & N/A \\
\hline & 4 & 0.143 & 0.103 & $0.167 \$$ & N/A \\
\hline & 5 & 0.642 & 0.230 & $0.005^{*}$ & N/A \\
\hline \multirow[t]{2}{*}{ Awareness DTC-GT disease } & Yes & 0.048 & 0.091 & 0.596 & N/A \\
\hline & No & Ref & & & \\
\hline \multirow[t]{2}{*}{ Awareness DTC-GT lifestyle } & Yes & -0.030 & 0.120 & 0.801 & N/A \\
\hline & No & Ref & & & \\
\hline How-to knowledge & $\begin{array}{l}\text { Per } 1 \text { point increase } \\
\text { in score }\end{array}$ & 0.010 & 0.026 & 0.696 & 0.240 \\
\hline Principles knowledge & $\begin{array}{l}\text { Per } 1 \text { point increase } \\
\text { in score }\end{array}$ & -0.026 & 0.015 & $0.082 \$$ & 0.784 \\
\hline Attitude & $\begin{array}{l}\text { Per } 1 \text { point increase } \\
\text { in score }\end{array}$ & 0.641 & 0.059 & $0.000^{*}$ & 0.350 \\
\hline Innovativeness & $\begin{array}{l}\text { Per } 1 \text { point increase } \\
\text { in score }\end{array}$ & 0.301 & 0.044 & $0.000^{*}$ & 0.313 \\
\hline
\end{tabular}


Chapter 2 | Acceptability, consideration and intention of DTC-GT uptake

\begin{tabular}{|c|c|c|c|c|c|}
\hline Consideration & & $\mathbf{b}$ & SE b & p-value & $\begin{array}{l}\text { Non-linearity } \\
\text { test }(\mathrm{p}) \#\end{array}$ \\
\hline \multirow[t]{2}{*}{ Gender } & Male & Ref & & & \\
\hline & Female & -0.006 & 0.075 & 0.938 & N/A \\
\hline Age in years & Per 1 year increase & -0.010 & 0.002 & $0.000^{*}$ & 0.366 \\
\hline \multirow[t]{3}{*}{ Education } & Low & Ref & & & \\
\hline & Medium & -0.016 & 0.087 & 0.854 & N/A \\
\hline & High & -0.156 & 0.100 & $0.119 \$$ & N/A \\
\hline \multirow[t]{2}{*}{ Having a partner } & Yes & 0.008 & 0.083 & 0.922 & N/A \\
\hline & No & Ref & & & \\
\hline \multirow[t]{2}{*}{ Being religious } & Yes & 0.039 & 0.075 & 0.605 & N/A \\
\hline & No & Ref & & & \\
\hline \multirow[t]{4}{*}{ Planning to have children } & Yes & 0.299 & 0.104 & $0.004^{*}$ & N/A \\
\hline & Maybe & 0.231 & 0.204 & 0.257 & N/A \\
\hline & Don't know & 0.301 & 0.179 & $0.093 \$$ & N/A \\
\hline & No & Ref & & & \\
\hline \multirow[t]{2}{*}{ Having biological children } & Yes & -0.185 & 0.077 & $0.016^{*}$ & N/A \\
\hline & No & Ref & & & \\
\hline \multirow{2}{*}{$\begin{array}{l}\text { Having adopted or step } \\
\text { children }\end{array}$} & Yes & 0.053 & 0.148 & 0.722 & N/A \\
\hline & No & Ref & & & \\
\hline \multirow[t]{3}{*}{ Genetic disease in the family } & Yes & 0.345 & 0.093 & $0.000^{*}$ & N/A \\
\hline & Don't know & 0.099 & 0.092 & 0.282 & N/A \\
\hline & No & Ref & & & \\
\hline \multirow[t]{2}{*}{ Having a chronic disease } & Yes & 0.105 & 0.078 & $0.177 \$$ & N/A \\
\hline & No & Ref & & & \\
\hline Self-rated health & $\begin{array}{l}\text { Per } 1 \text { point increase } \\
\text { in score }\end{array}$ & 0.113 & 0.043 & $0.009^{*}$ & 0.311 \\
\hline \multirow[t]{2}{*}{ Awareness DTC-GT disease } & Yes & 0.070 & 0.083 & 0.394 & N/A \\
\hline & No & Ref & & & \\
\hline \multirow[t]{2}{*}{ Awareness DTC-GT lifestyle } & Yes & 0.132 & 0.109 & 0.226 & N/A \\
\hline & No & Ref & & & \\
\hline How-to knowledge & $\begin{array}{l}\text { Per } 1 \text { point increase } \\
\text { in score }\end{array}$ & -0.055 & 0.024 & $0.021^{*}$ & 0.505 \\
\hline Principles knowledge & $\begin{array}{l}\text { Per } 1 \text { point increase } \\
\text { in score }\end{array}$ & 0.011 & 0.013 & 0.426 & 0.485 \\
\hline Attitude & $\begin{array}{l}\text { Per } 1 \text { point increase } \\
\text { in score }\end{array}$ & 0.843 & 0.049 & $0.000^{*}$ & 0.425 \\
\hline Innovativeness & $\begin{array}{l}\text { Per } 1 \text { point increase } \\
\text { in score }\end{array}$ & 0.566 & 0.036 & $0.000^{*}$ & 0.502 \\
\hline
\end{tabular}


Chapter 2 | Acceptability, consideration and intention of DTC-GT uptake

\begin{tabular}{|c|c|c|c|c|c|}
\hline Intention & & $\mathbf{b}$ & SE b & p-value & $\begin{array}{l}\text { Non-linearity } \\
\text { test }(\mathrm{p}) \#\end{array}$ \\
\hline \multirow[t]{2}{*}{ Gender } & Male & Ref & & & \\
\hline & Female & 0.024 & 0.068 & 0.719 & N/A \\
\hline Age in years & Per 1 year increase & -0.005 & 0.002 & $0.017^{*}$ & 0.318 \\
\hline \multirow[t]{3}{*}{ Education } & Low & Ref & & & \\
\hline & Medium & 0.003 & 0.078 & 0.966 & N/A \\
\hline & High & -0.191 & 0.091 & $0.035^{*}$ & N/A \\
\hline \multirow[t]{2}{*}{ Having a partner } & Yes & -0.048 & 0.075 & 0.527 & N/A \\
\hline & No & Ref & & & \\
\hline \multirow[t]{2}{*}{ Being religious } & Yes & 0.0405 & 0.069 & 0.555 & N/A \\
\hline & No & Ref & & & \\
\hline \multirow[t]{4}{*}{ Planning to have children } & Yes & 0.048 & 0.095 & 0.609 & N/A \\
\hline & Maybe & 0.173 & 0.186 & 0.352 & N/A \\
\hline & Don't know & 0.084 & 0.164 & 0.607 & N/A \\
\hline & No & Ref & & & \\
\hline \multirow[t]{2}{*}{ Having biological children } & Yes & -0.114 & 0.070 & $0.101 \$$ & N/A \\
\hline & No & Ref & & & \\
\hline \multirow{2}{*}{$\begin{array}{l}\text { Having adopted or step } \\
\text { children }\end{array}$} & Yes & -0.041 & 0.135 & 0.760 & N/A \\
\hline & No & Ref & & & \\
\hline \multirow[t]{3}{*}{ Genetic disease in the family } & Yes & 0.150 & 0.085 & $0.077 \$$ & N/A \\
\hline & Don't know & 0.052 & 0.084 & 0.537 & N/A \\
\hline & No & Ref & & & \\
\hline \multirow[t]{2}{*}{ Having a chronic disease } & Yes & 0.089 & 0.070 & 0.208 & N/A \\
\hline & No & Ref & & & \\
\hline Self-rated health & $\begin{array}{l}\text { Per } 1 \text { point increase } \\
\text { in score }\end{array}$ & 0.127 & 0.039 & $0.001^{*}$ & 0.644 \\
\hline \multirow[t]{2}{*}{ Awareness DTC-GT disease } & Yes & -0.009 & 0.075 & 0.908 & N/A \\
\hline & No & Ref & & & \\
\hline \multirow[t]{2}{*}{ Awareness DTC-GT lifestyle } & Yes & 0.045 & 0.099 & 0.649 & N/A \\
\hline & No & Ref & & & \\
\hline How-to knowledge & $\begin{array}{l}\text { Per } 1 \text { point increase } \\
\text { in score }\end{array}$ & -0.090 & 0.021 & $0.000^{*}$ & 0.294 \\
\hline Principles knowledge & $\begin{array}{l}\text { Per } 1 \text { point increase } \\
\text { in score }\end{array}$ & -0.015 & 0.012 & 0.220 & 0.948 \\
\hline Attitude & $\begin{array}{l}\text { Per } 1 \text { point increase } \\
\text { in score }\end{array}$ & 0.454 & 0.049 & $0.000^{*}$ & 0.364 \\
\hline Innovativeness & $\begin{array}{l}\text { Per } 1 \text { point increase } \\
\text { in score }\end{array}$ & 0.417 & 0.034 & $0.000^{*}$ & 0.371 \\
\hline
\end{tabular}

Legend: ${ }^{*} \alpha<0.05 ; \$ 0.05<\alpha<0.20$; \#If the linearity test was significant at $\alpha<0.05$, an additional univariate analysis was done with the variable as categorical variable and was entered in the multivariate analyses as a categorical variable. 
Chapter 2 | Acceptability, consideration and intention of DTC-GT uptake 


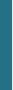




\section{Lay summary}

Smoking is a key risk factor for the development of chronic obstructive pulmonary disease (COPD) but not every smoker develops this disease. This means that it is likely that other factors, like other lifestyle factors, environment and genetics, also play a role in the development of this disease. In this study our objective was to summarize the latest scientific findings on these topics published at a high level of evidence (namely, meta-analyses). We identified 204 different genetic variants (small alterations in the genetic code which naturally exist between people), which increased or decreased the risk of developing COPD. Additionally, we found six lifestyle and environmental factors that contributed to the development of COPD: smoking, second-hand tobacco smoke, unhealthy diet, occupational exposure to endotoxins and vapours, gases, dusts or fumes, as well as poor air quality measured as living in a polluted area and the use of solid fuels for heating or cooking indoors. However, it is worth noting that in some instances risk carried by specific genetic factors was only present in combination with or in absence of specific secondary factors such as smoking or ethnicity. Thus, genetic, lifestyle and environmental factors come together to result in a disease. These results can help researchers to explore the way COPD develops and help set up strategies for prevention, for example through lifestyle interventions in which important risk factors are targeted. 


\section{Abstract}

Rationale: Although smoking is the key risk factor for chronic obstructive pulmonary disease (COPD), not all smokers develop it. Lifestyle, environmental and genetic factors may play a role in the etiology.

Objectives: Our aim was to summarize the current state of knowledge, at the highest level of evidence, on factors associated with development of COPD.

Methods: Relevant meta-analyses were identified via MedLine, Web of Science and EMBASE. Meta-analyses were included if they reported genetic variants, lifestyle or environmental factors associated with COPD, emphysema or chronic bronchitis.

Measurements: For each risk factor - disease combination the most comprehensive odds ratio $(\mathrm{OR})$ was determined by either calculation of a meta-OR through metameta-analysis, or by selecting the most comprehensive OR based on the most recent and/or complete meta-analysis.

Main results: For genetic and lifestyle/environmental factors respectively, 64 and 19 publications were identified and 47 and ten were selected as most comprehensive. Of the 274 genetic variants identified, $74 \%(n=204)$ showed a significant association with COPD (OR range: 0.17-3.33). Significant associations were found for six of seven identified lifestyle/environmental factors (ORs range: $0.45-9.50$ ).

Conclusion: Our report provides the first up-to-date and complete overview of genetic, lifestyle and environmental risk factors of COPD. 


\section{Introduction}

Chronic obstructive pulmonary disease (COPD) is a chronic respiratory disease characterized by being a mostly irreversible and typically progressive reduction in airflow ${ }^{1}$. Worldwide, it is estimated that $11.7 \%$ of people aged 30 and over suffer from the disease ${ }^{2}$ and approximately $5 \%$ of all deaths is attributable to COPD; making it one of the leading causes of death ${ }^{3}$.

The most important risk factor for COPD is tobacco smoking ${ }^{4,5}$, increasing the risk by over three-fold 6 . However, not all smokers develop COPD, only about $20 \%$ of smokers will ever develop the disease ${ }^{7}$, and furthermore not every COPD patient has a history of smoking behavior 5 . Other typical non-genetic risk factors include the use of solid fuel for cooking and heating in poorly ventilated houses, as well as exposure to gases or fumes, mostly from occupational exposure. ${ }^{8-10}$ In addition, a great number of genetic variants have been identified that were found to be associated with development of COPD.

With the large amount of evidence aggregated over the years regarding risk factors of COPD, a comprehensive summary at the highest level of evidence will be helpful to different end-users. To date, no such report has been published, incorporating genetic, lifestyle and environmental factors of COPD risk. Such an overview can help set up effective preventive strategies, screening practices, identifying knowledge gaps and supporting the work of future researchers. Therefore it is our aim to give an overview of the current evidence of genetic, lifestyle and environmental factors associated with the development of COPD, as researched in meta-analyses.

\section{Methods}

Prior to starting the research, a protocol was registered in PROSPERO (CRD42017051582).

\section{Search strategy}

Databases of MedLine, Web of Science, and EMBASE were searched from inception up to May 2018, with no restrictions to language or publication year. The following simplified search strategies were used: (COPD OR emphysema OR chronic bronchitis) AND (Genome wide association study OR candidate gene studies OR polymorphism) for genetic factors, and (COPD OR emphysema OR chronic bronchitis) AND (diet OR dietary OR nutrition * OR exercise OR "physical activity" OR smoking OR tobacco OR environment* 
OR occupation* OR alcohol OR alcoholic) AND ("Meta-Analysis" OR "pooled analysis") for lifestyle and environmental factors. The full search strategies can be found in appendix 1.

\section{Eligibility criteria}

Studies were eligible for inclusion when they reported genetic variants, lifestyle factors, or environmental factors associated with COPD, emphysema (EM) or chronic bronchitis (CB) risk in meta-analysis.

\section{Study selection}

After removal of duplicates, publications were screened by title and abstract. Second selection was based on full-text versions and done by SM and KS jointly. Disagreement was discussed until consensus was reached.

\section{Data extraction}

The following data was extracted: title, author, year, number of included primary studies, number of cases and controls, ethnicity, sex, relative risks per risk factor-disease combination (overall and subgroup), heterogeneity, and publication bias. Data was extracted by one researcher and checked for errors by a second researcher. Authors of publications were contacted for additional information in case of uncertainties.

\section{Selection criteria for estimates}

To be included, each meta-analytic estimate had to be based on a minimum of two primary studies. They were selected in the following order: (1) overall over sub-group estimates, (2) more adjusted over less adjusted estimates, (3) smoking adjustment over other adjustments, (4) incidence over prevalence, (5) random-effects model over fixedeffects model. Mortality as an outcome was only accepted if combined into one estimate with incidence and/or prevalence. Meta-analyses including both case-control and cohort studies were accepted.

\section{Methodological quality}

The Assessment of Multiple Systematic Reviews (AMSTAR), was used to appraise methodological quality of the individual meta-analyses. ${ }^{11}$ Each included publication was rated separately by SM and KS and disagreements were solved through discussion. If consensus could not be reached AW was consulted. The assessment was used for evaluative purposes only.

\section{Data synthesis}

If only one meta-analysis was available on a specific factor, these estimates were included 
directly. If multiple Meta-analyses were available, the most comprehensive estimate was included. This could occur in one of two ways: (1) Meta-analyses were combined in meta-meta-analysis to obtain an overall estimate based on the largest number of primary data. This was only considered appropriate if $\leq 50 \%$ of the studies of the smaller publication overlapped with those of the largest population. If this made meta-metaanalysis inappropriate, (2) the most comprehensive meta-analysis was included and secondly on the largest number of primary studies included. If this number was equal, the publication with the largest total number of participants was used. Meta-metaanalysis was performed using a random-effects model and heterogeneity was explored using the $\mathrm{I}^{2}$-statistic. ${ }^{12}$ All analyses were done using Stata 14 (Statacorp LP). ORs are referred to as $\mathrm{OR}_{\mathrm{COPD}}, \mathrm{OR}_{\mathrm{EM}}$, and $\mathrm{OR}_{\mathrm{CB}}$ for $\mathrm{COPD}, \mathrm{EM}$, and $\mathrm{CB}$ respectively. ORs from meta-meta-analysis will be referred to as a meta-OR ( $\mathrm{mOR}$ ).

Extracted estimates for genetic factors were categorized under their appropriate model: allelic (e.g. A vs. T), dominant (e.g. AA+AT vs. TT), recessive (e.g. AA vs. AT+TT), homozygote (e.g. AA vs. TT), heterozygote (e.g. AT vs. TT), over-dominant (e.g. AT vs. $\mathrm{AA}+\mathrm{TT}$ ), and 'other' (e.g. slow vs. normal enzyme activity).

\section{Attributable risk percent and preventive fraction}

To determine targets for preventive strategies, amongst other purposes, attributable risk percent $(\mathrm{AR} \%)$ was calculated for modifiable risk factors that were significantly associated with COPD, CB and EM. The AR\% can be interpreted as the potentially preventable percentage of disease incidence among the exposed that are the result of the exposure, and therefore could be prevented if the exposure were eliminated ${ }^{13}$. In case of protective factors $(R R<1.00)$, the $R R$ was inversed to represent the risk associated with absence of this factor. ORs were used as an approximation of the RR if RRs were not available.

\section{Results}

\section{Literature search}

The literature search is depicted in Figures 1 and 2 for genetic and lifestyle/environmental factors respectively. A total of 64 and 19 publications were selected to be included in our study for genetic and lifestyle/environmental factors respectively. Of these, 47 and ten publications were selected as most comprehensive for at least one risk factor. Metameta-analysis was performed for two genetic and four lifestyle/environmental factors. Supplementary tables 1 and 2 present the selected publications, including for which factors they have been selected as most comprehensive. 


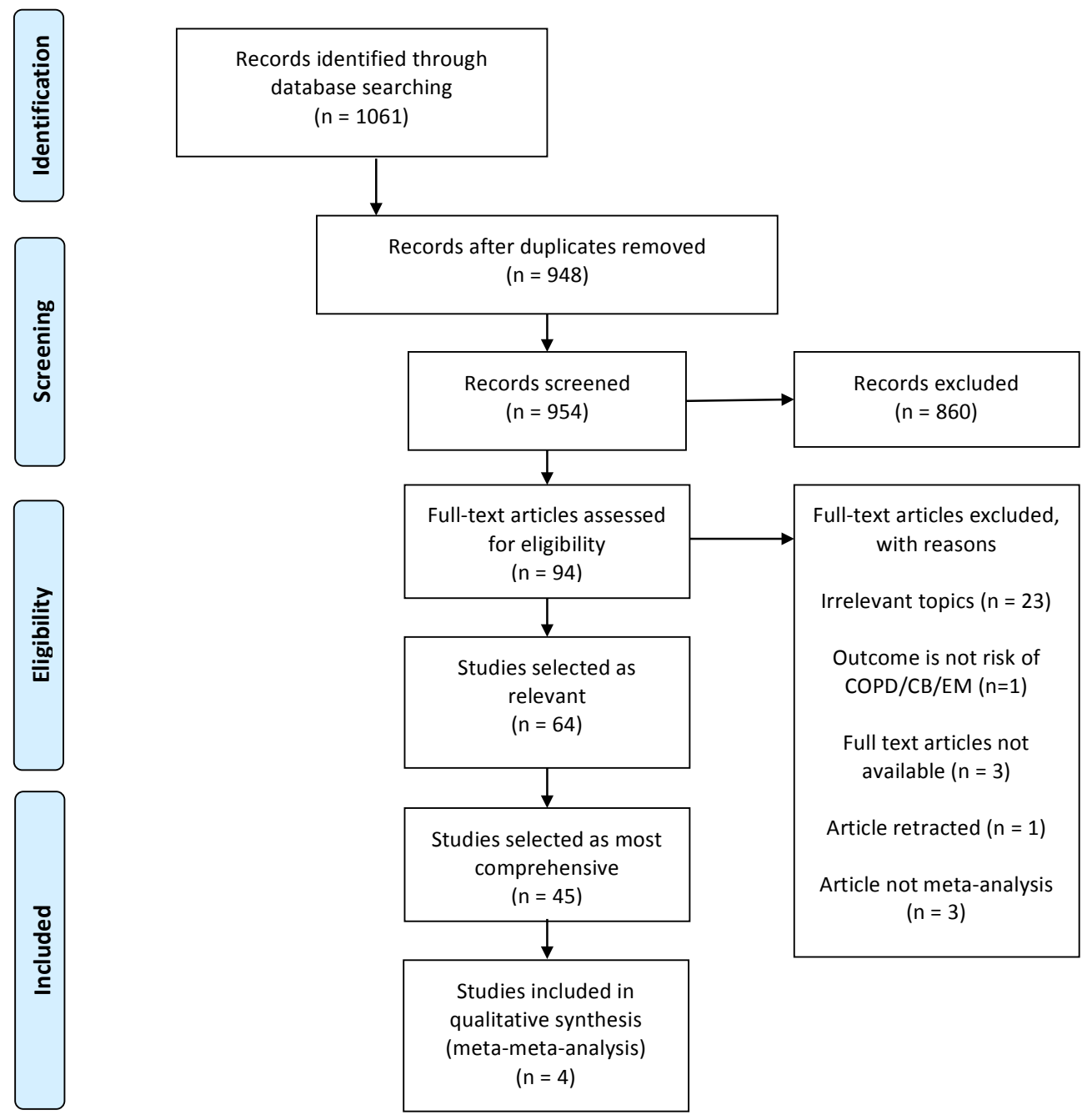

Figure 1 Flow diagram for genetic factors associated with COPD

\section{Reporting quality}

Overall, AMSTAR ratings varied widely: $1 / 11$ to $8 / 11$ for meta-analyses and $0 / 4$ to $3 / 4$ for pooled analyses. This implies that the quality of the meta-analyses can be improved significantly. An overview of scores per item are visualized in Supplementary figure 1 for genetic and lifestyle/environmental factors respectively.

\section{Genetic factors}

Of the 274 single nucleotide polymorphisms (SNPs) or SNP combinations identified, $74 \%(n=204)$ showed a significant association for either COPD, emphysema and/or 


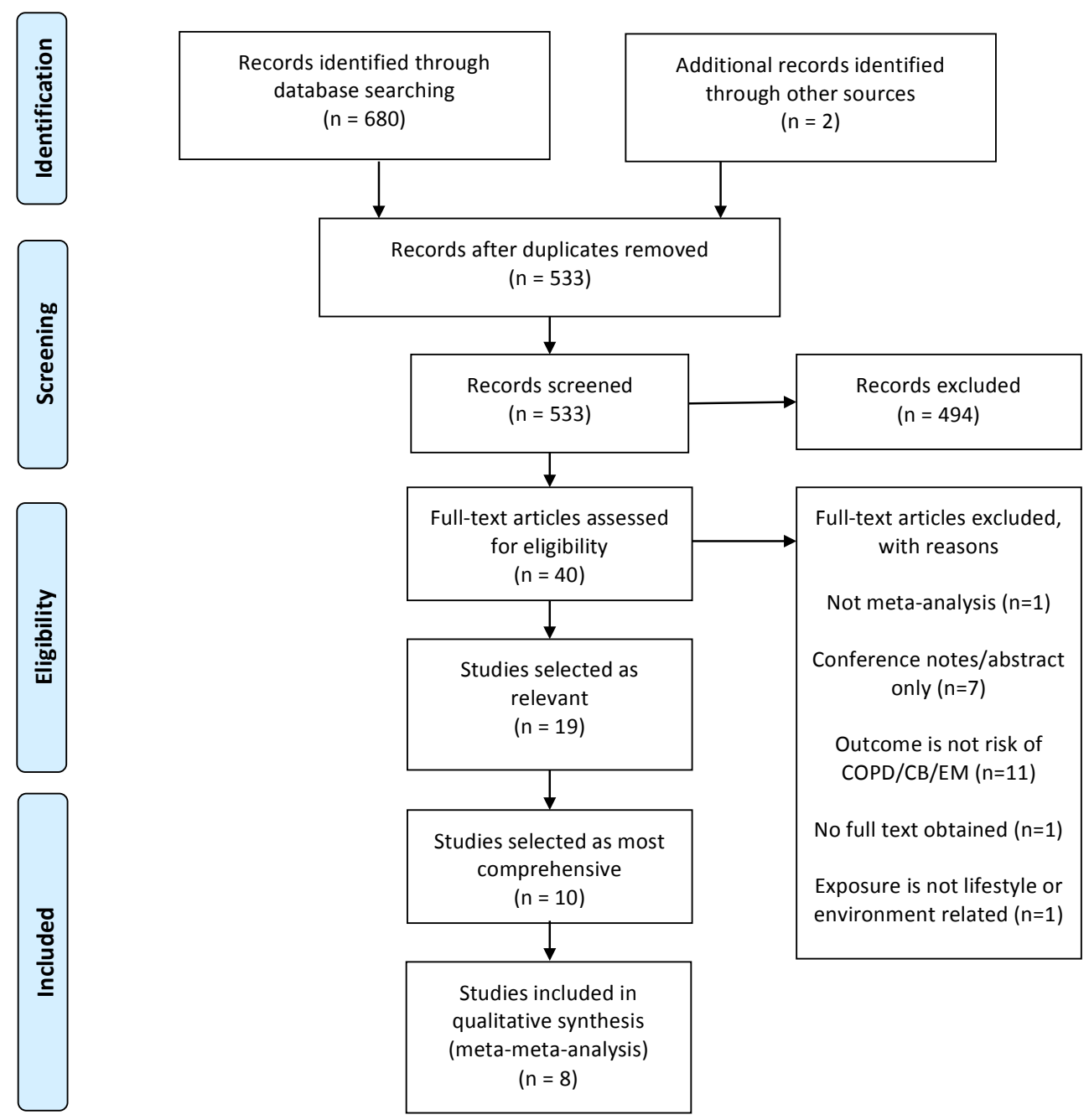

Figure 2 Flow diagram for lifestyle factors associated with COPD

chronic bronchitis. Two meta-meta-analyses were performed, for CHRNA5 rs16969968 and IREB2 rs2568494. Both associations were statistically significant with meta-ORs of $1.30(1.24-1.36)$ and $0.85(0.76-0.93)$ respectively under the allelic model. Due to the large amount of information, only those variants with significant ORs $\leq 0.50$ or $\geq 2.00$ will be discussed and are displayed in table 1. Variants with significant associations of $0.5>\mathrm{OR}<2.0$ and variants that were not significantly associated are displayed in Supplementary tables 3 and 4 respectively. 
Chapter 3 | Genetic, lifestyle, and environmental risk factors of COPD development

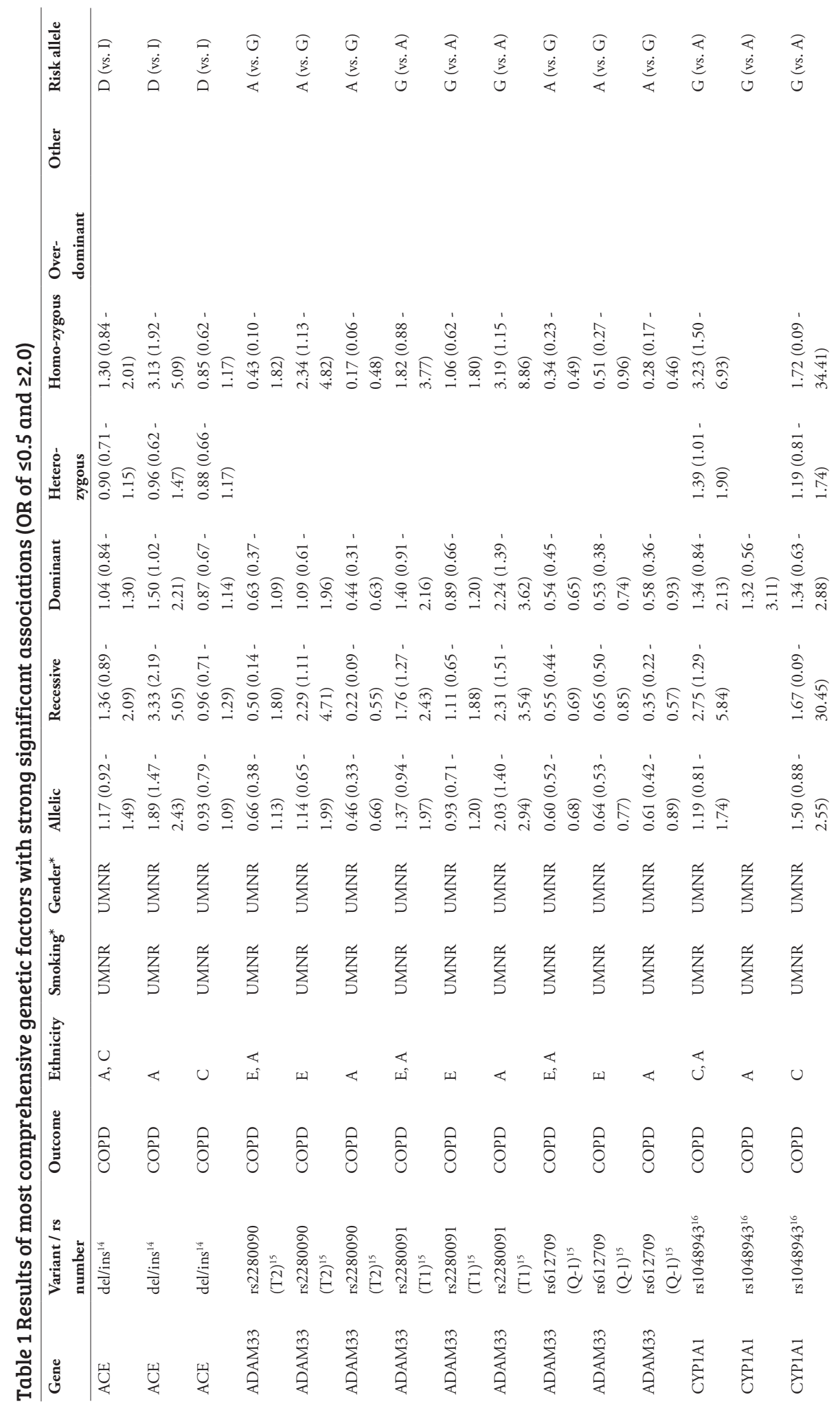


Chapter 3 | Genetic, lifestyle, and environmental risk factors of COPD development

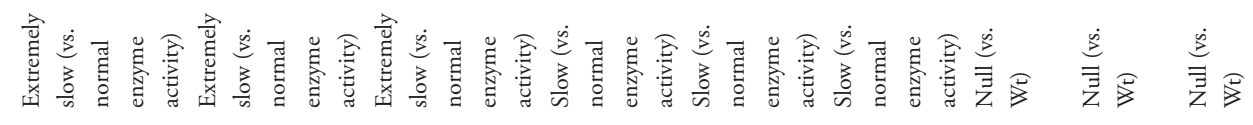

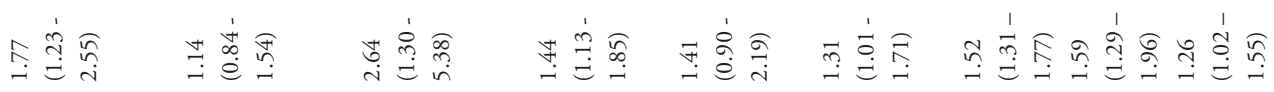

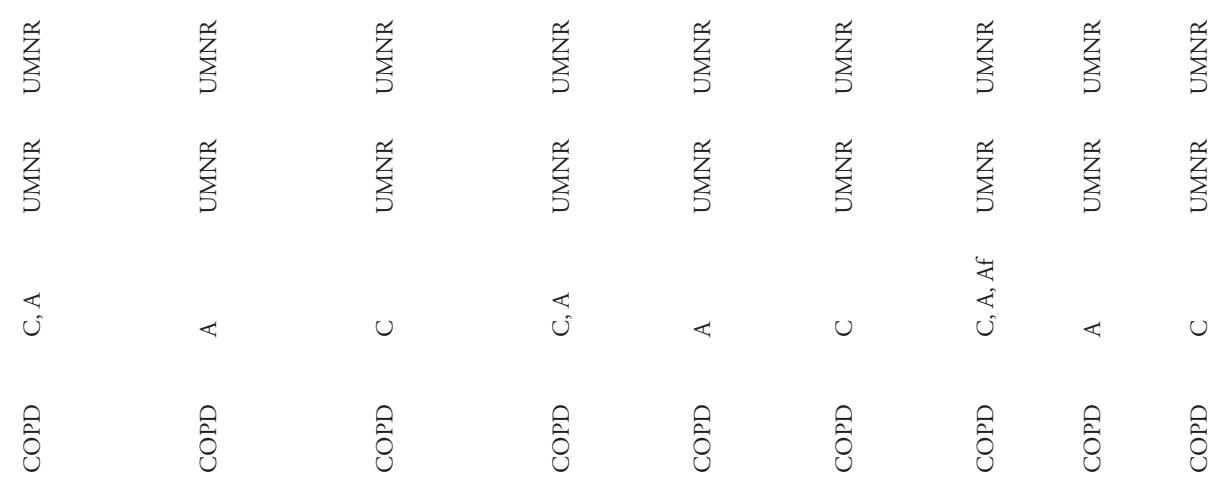

WhMWMn Wh

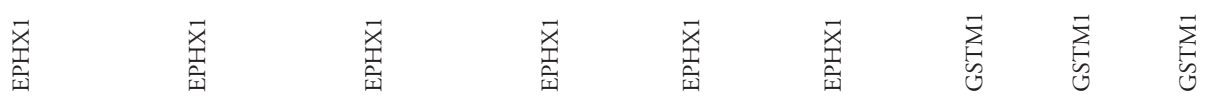


Chapter 3 | Genetic, lifestyle, and environmental risk factors of COPD development

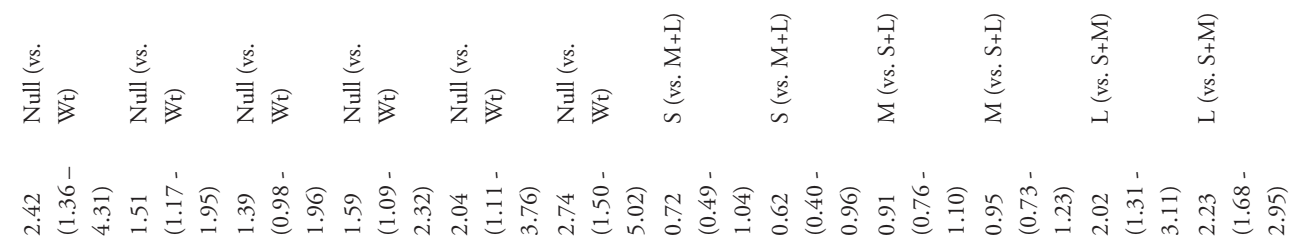

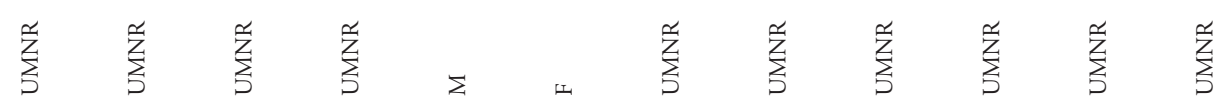

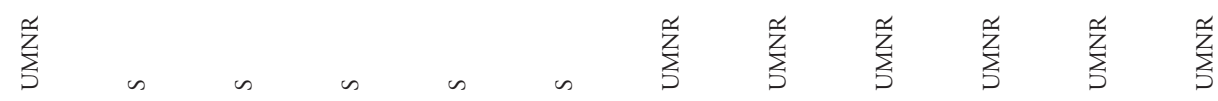

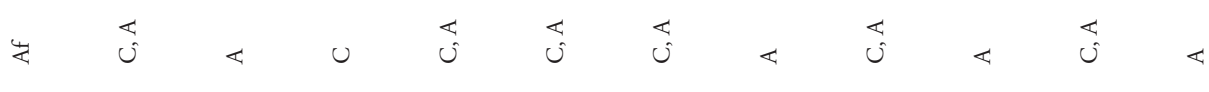

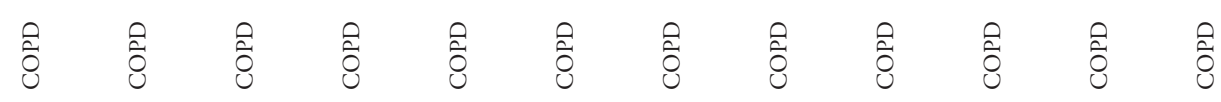

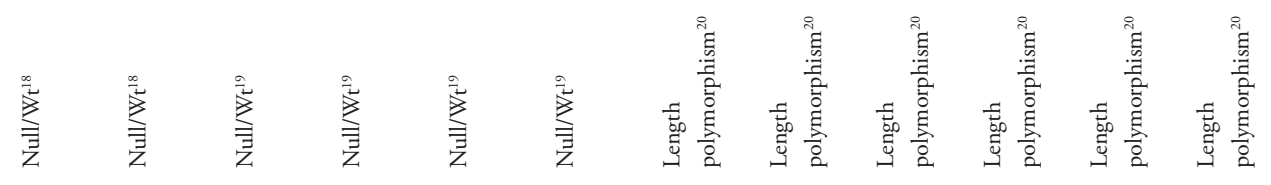

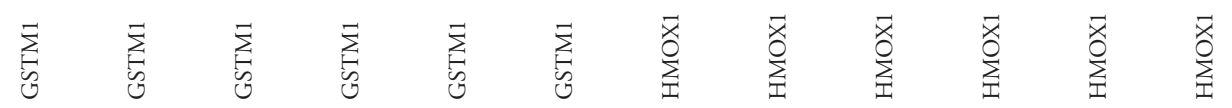


Chapter 3 | Genetic, lifestyle, and environmental risk factors of COPD development

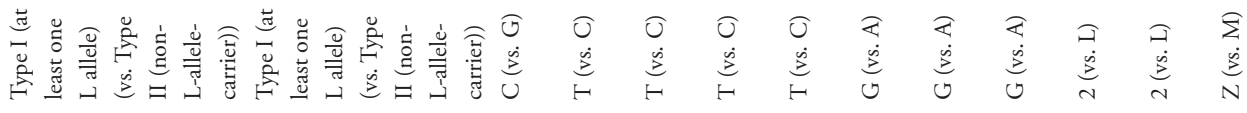

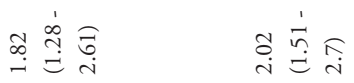

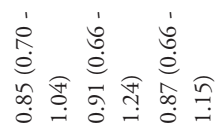

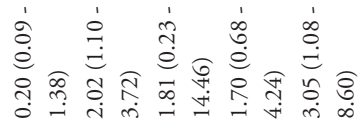

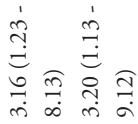

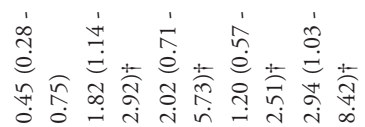

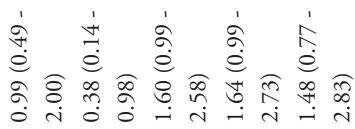

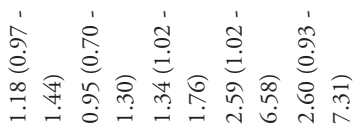

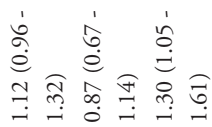

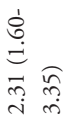

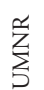

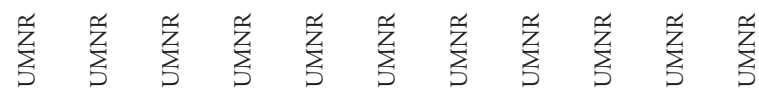

$\sum_{j}^{\infty} \quad \sum_{j}^{a}$

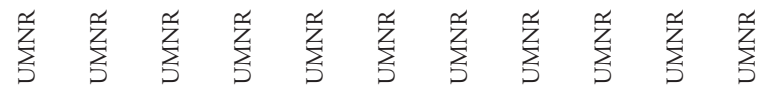

us $\quad$ u

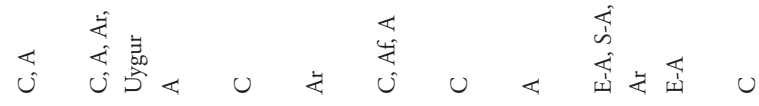

ชิ

ठิ

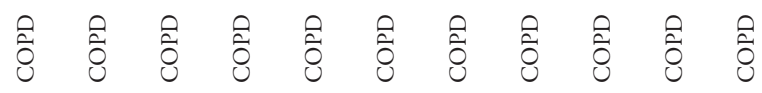

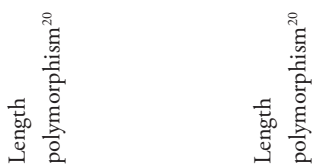


Chapter 3 | Genetic, lifestyle, and environmental risk factors of COPD development

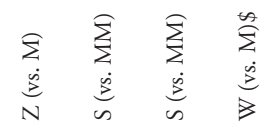

$\sum_{\substack{\infty \\ \sum}}^{\infty}$

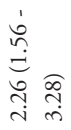

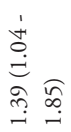

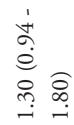

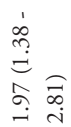

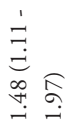

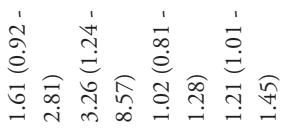

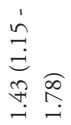

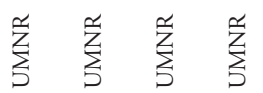

$\sum_{j}^{\infty}$

i $\sum_{j}^{n}$ is $\sum_{5}^{\frac{\sigma}{z}}$

$\sum_{5}^{\infty}$

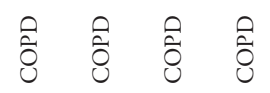

ิิ

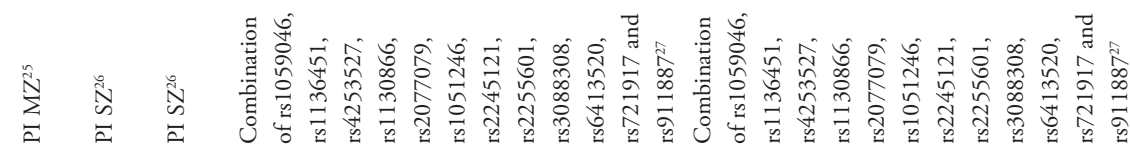

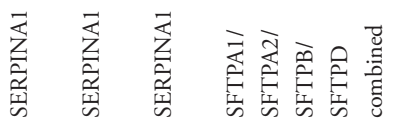

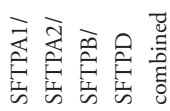


Chapter 3 | Genetic, lifestyle, and environmental risk factors of COPD development

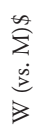

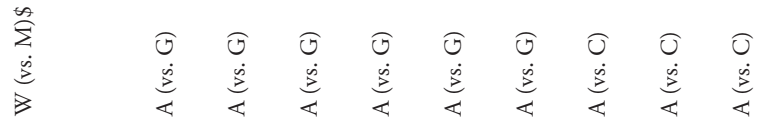

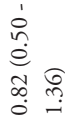

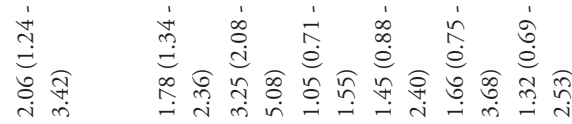

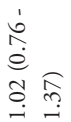

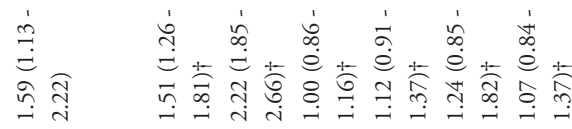

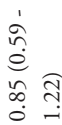

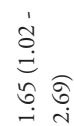

กั

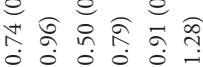

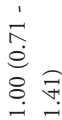

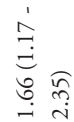

হ̇̀

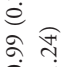

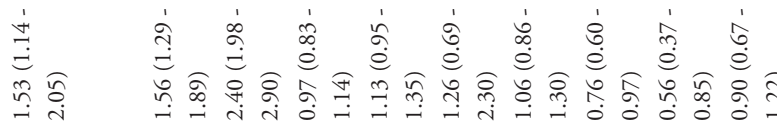

$\sum_{5}^{\infty}$

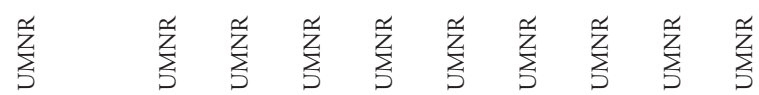

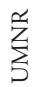

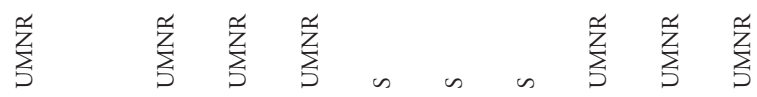

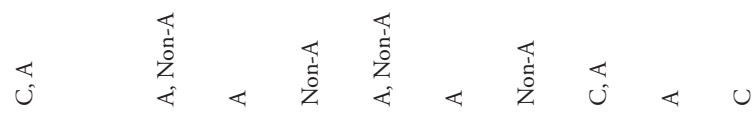

ิิ

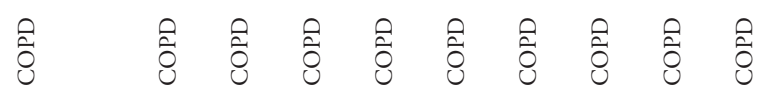

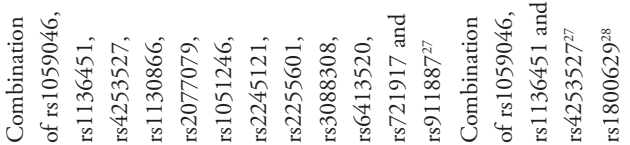

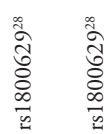

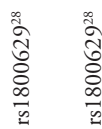

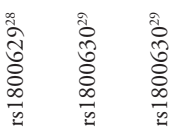

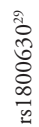

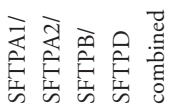

空密

崖崖崖崖崖崖崖崖崖 
Chapter 3 | Genetic, lifestyle, and environmental risk factors of COPD development

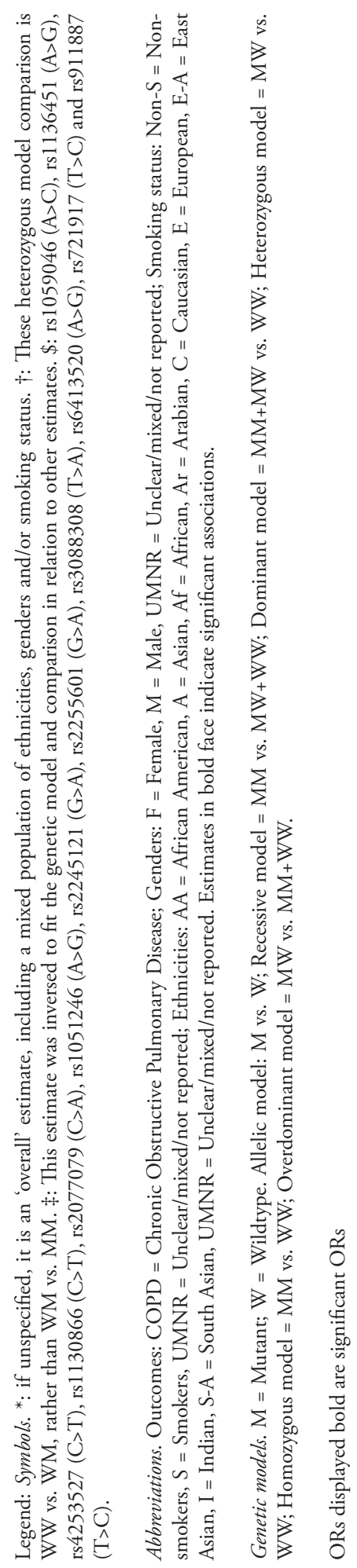




\section{Angiotensin I converting enzyme (ACE)}

The insertion/deletion polymorphism increased the COPD risk in Asians, but not in Europeans. ${ }^{14}$ The risk was particularly large for homozygous models $\left(\mathrm{OR}_{\mathrm{COPD}}=3.33\right.$, recessive model).$^{14}$

\section{ADAM metallopeptidase domain 33 (ADAM33)}

Many variants of the ADAM33 gene were associated with COPD, with particularly strong associations for rs2280090 (T2), rs2280091 (T1), and rs612709 (Q-1). ${ }^{15}$ Rs2280090 (T2) increased the risk (e.g. $\mathrm{OR}_{\mathrm{COPD}}=2.34$, homozygous model) for Europeans, whereas the risk was decreased for Asians (e.g. $\mathrm{OR}_{\mathrm{COPD}}=0.17$, homozygous model). Rs2280091 (T1) only showed an increased risk among Asians $\left(\mathrm{OR}_{\mathrm{COPD}}=3.19\right.$, homozygous model $)$. Rs612709 (Q-1) decreased the risk among both Europeans and Asians under all models (e.g. $\mathrm{OR}_{\mathrm{COPD}}=0.51$ and $\mathrm{OR}_{\mathrm{COPD}}=0.28$ respectively, homozygous model).

\section{Cytochrome P450 family 1 subfamily A member 1 (CYP1A1)}

The risk of COPD was particularly increased for rs1048943, but only when both ethnicities were combined (e.g. $\mathrm{OR}_{\mathrm{COPD}}=2.75$ and $\mathrm{OR}_{\mathrm{COPD}}=3.23$ for recessive and overdominant models respectively). ${ }^{16}$

\section{Epoxide hydrolase 1 (EPHXI)}

Extremely slow activity EPHX1 encoded enzyme hydrolase 1 (determined by a combination of rs 1051740 and rs2234922) resulted in an increased COPD risk in both the overall and Caucasian-specific estimates $\left(\mathrm{OR}_{\mathrm{COPD}}=1.77\right.$ and $\mathrm{OR}_{\mathrm{COPD}}=2.64$ respectively), but not in Asians. ${ }^{17}$

\section{Glutathione S-transferase mu 1 (GSTM1)}

The null genotype of GSTM1 increased COPD risk for all ethnicities combined $\left(\mathrm{OR}_{\mathrm{COPD}}=1.52\right.$, allelic model $)$, but was particularly high for Africans $\left(\mathrm{OR}_{\mathrm{COPD}}=2.42\right.$, allelic model) and Asians $\left(\mathrm{OR}_{\mathrm{COPD}}=1.59\right.$, allelic model), in contrast to Caucasians $\left(\mathrm{OR}_{\mathrm{COPD}}=1.26\right.$, allelic model $) .{ }^{18}$ Restricted to current and former smokers, estimates were similar $\left(\mathrm{OR}_{\mathrm{COPD}}=1.51\right.$ and $\mathrm{OR}_{\mathrm{COPD}}=1.59$ for overall and Caucasians respectively, allelic model)(19). The risk was slightly higher for female than for male smokers $\left(\mathrm{OR}_{\mathrm{COPD}}=2.74\right.$ and $\mathrm{OR}_{\mathrm{COPD}}=2.04$ respectively, allelic model $)$.

\section{Heme oxygenase 1 (HMOX1)}

Long (L) allele carriers of the HMOX1 gene had an increased COPD risk compared to short $(\mathrm{S})$ and medium $(\mathrm{M})$ allele carriers (e.g. $\mathrm{OR}_{\mathrm{COPD}}=2.02$ for people with at least one L-allele compared to non-L-allele carriers). ${ }^{20}$ 
Interleukin 6 (IL6)

COPD risk was particularly decreased for rs1800796 (e.g. $\mathrm{OR}_{\mathrm{COPD}}=0.20$, homozygous model). ${ }^{21}$

Interleukin 13 (IL13)

Rs1800925 increased the COPD risk in the overall estimate $\left(\mathrm{OR}_{\mathrm{COPD}}=1.82\right.$ and $\mathrm{OR}_{\mathrm{COPD}}=2.02$ heterozygous and homozygous models respectively) and specifically for Arabians $\left(\mathrm{OR}_{\mathrm{COPD}}=2.94\right.$ and $\mathrm{OR}_{\mathrm{COPD}}=3.05$ heterozygous and homozygous models respectively). ${ }^{22}$ Rs20541 decreased the risk in Caucasians $\left(\mathrm{OR}_{\mathrm{COPD}}=0.38\right.$, dominant model) whereas it increased the risk in Asians $\left(\mathrm{OR}_{\mathrm{COPD}}=1.30\right.$, allelic model) ${ }^{23}$

\section{Interleukin 1 receptor antagonist (IL1RN)}

The COPD risk was increased by rs2234663 in all ethnicities combined $\left(\mathrm{OR}_{\mathrm{COPD}}=2.59\right.$ and $\mathrm{OR}_{\mathrm{COPD}}=3.16$, recessive and homozygous models respectively). A similar risk was seen for East-Asians $\left(\mathrm{OR}_{\mathrm{COPD}}=3.20\right.$, homozygous model $){ }^{24}$

\section{Serpin family A member 1 (SERPINA1)}

The $\mathrm{Z}$ allele of SERPINA1, compared to the $\mathrm{M}$ allele, increased the COPD risk by over twofold $\left(\mathrm{OR}_{\mathrm{COPD}}=2.31\right)$ but this association disappeared after adjustment for smoking. ${ }^{25}$ Compared to MM homozygotes, SZ heterozygotes had an increased risk of over threefold $\left(\mathrm{OR}_{\mathrm{COPD}}=3.26\right) .^{26}$

\section{Surfactant protein A1/A2/B/D (SFTPA1/A2/B/D)}

All variants of SFTPA1/A2/B/D combined led to an increased COPD risk for Asians $\left(\mathrm{OR}_{\mathrm{COPD}}=1.97\right.$ and $\mathrm{OR}_{\mathrm{COPD}}=2.26$ for dominant and homozygous models respectively $)$ but not Caucasians. ${ }^{27}$

\section{Tumor necrosis factor (TNF)}

Rs1800629 increased the COPD risk for Asians (e.g. $\mathrm{OR}_{\mathrm{COPD}}=2.40$, allelic model) but not for non-Asians. ${ }^{28}$ Restricted to smokers only, this association disappeared. Rs 1800630 reduced the COPD risk in Asians only (e.g. $\mathrm{OR}_{\mathrm{COPD}}=0.50$, dominant model). ${ }^{29}$

\section{Lifestyle and environmental factors}

Publications were available on nine different lifestyle (Table 2) and environmental factors (Table 3). Only significant associations are discussed below. 
Chapter 3 | Genetic, lifestyle, and environmental risk factors of COPD development

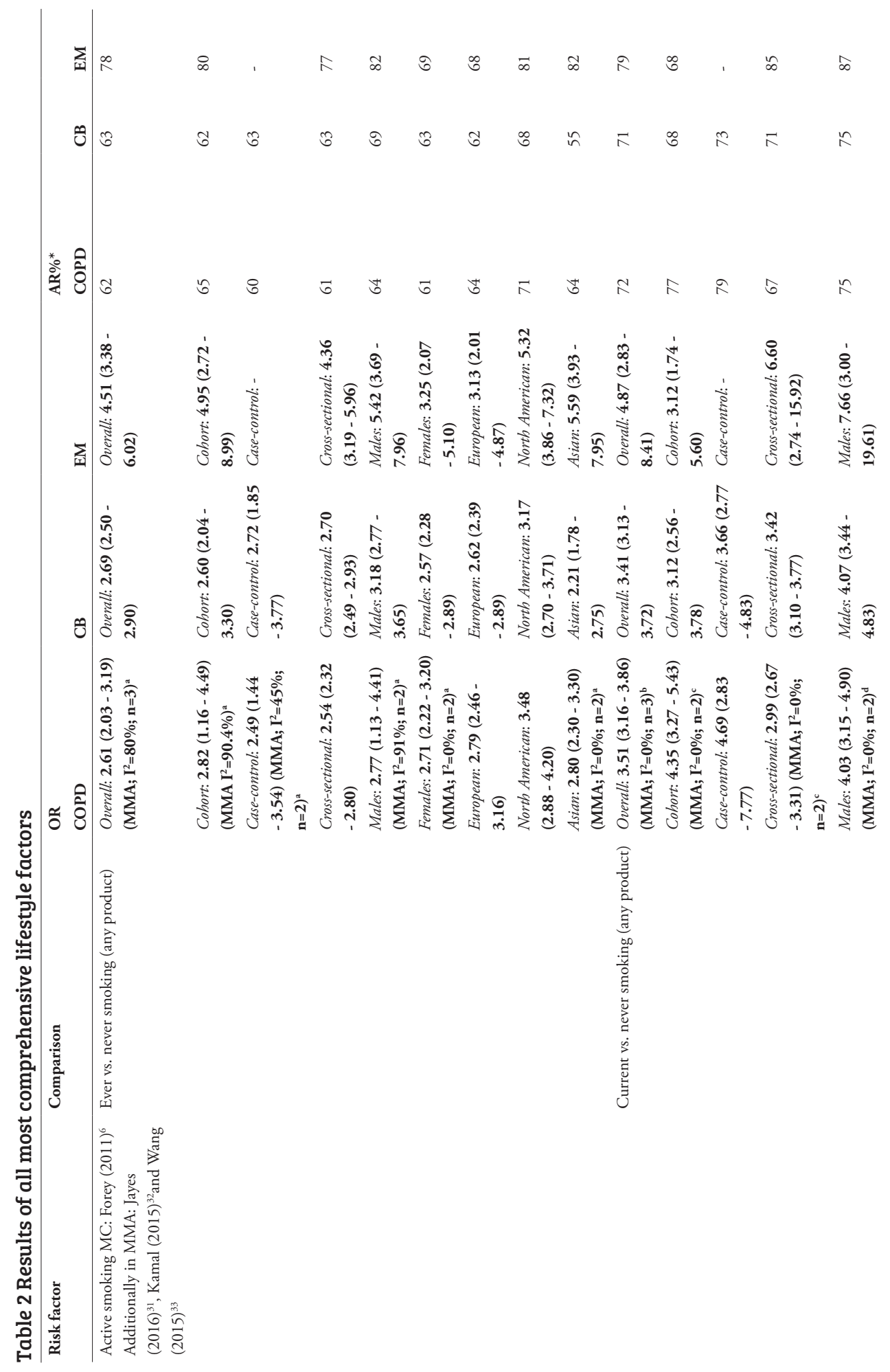


Chapter 3 | Genetic, lifestyle, and environmental risk factors of COPD development

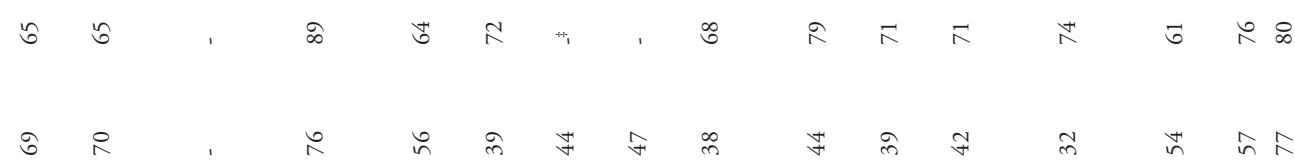

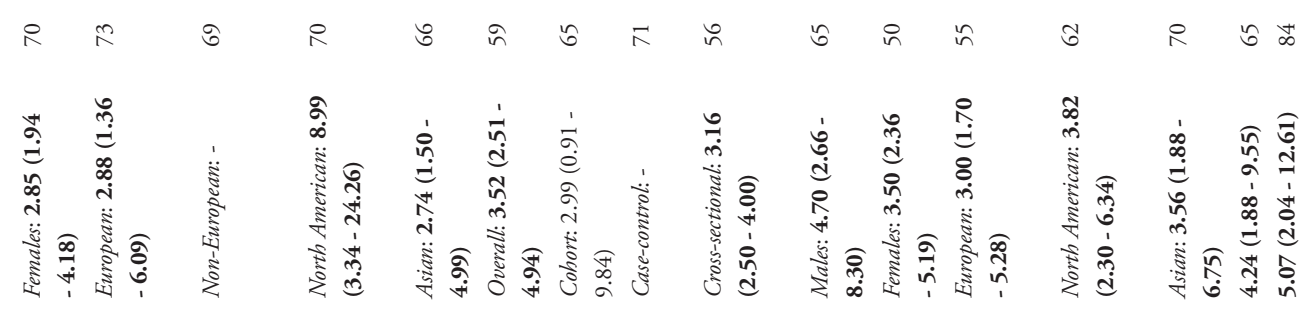

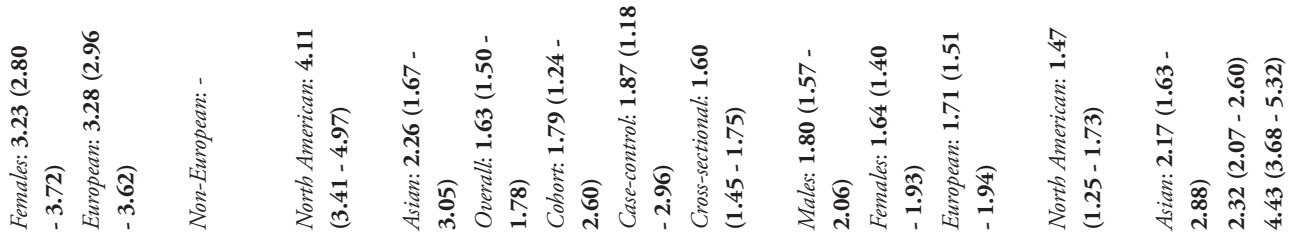

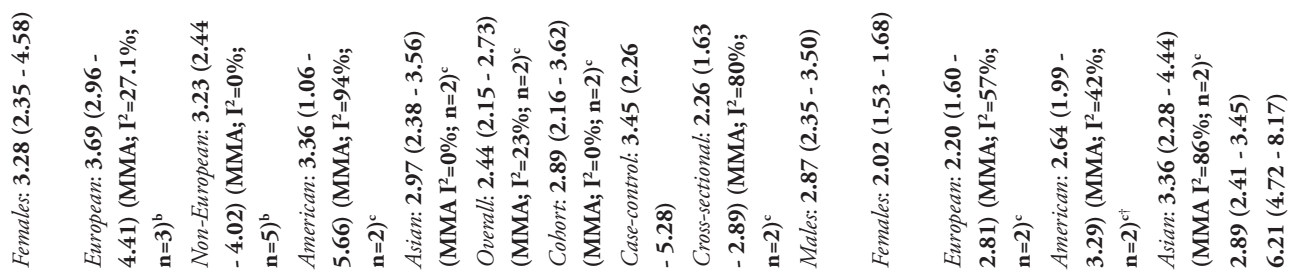


Chapter 3 | Genetic, lifestyle, and environmental risk factors of COPD development

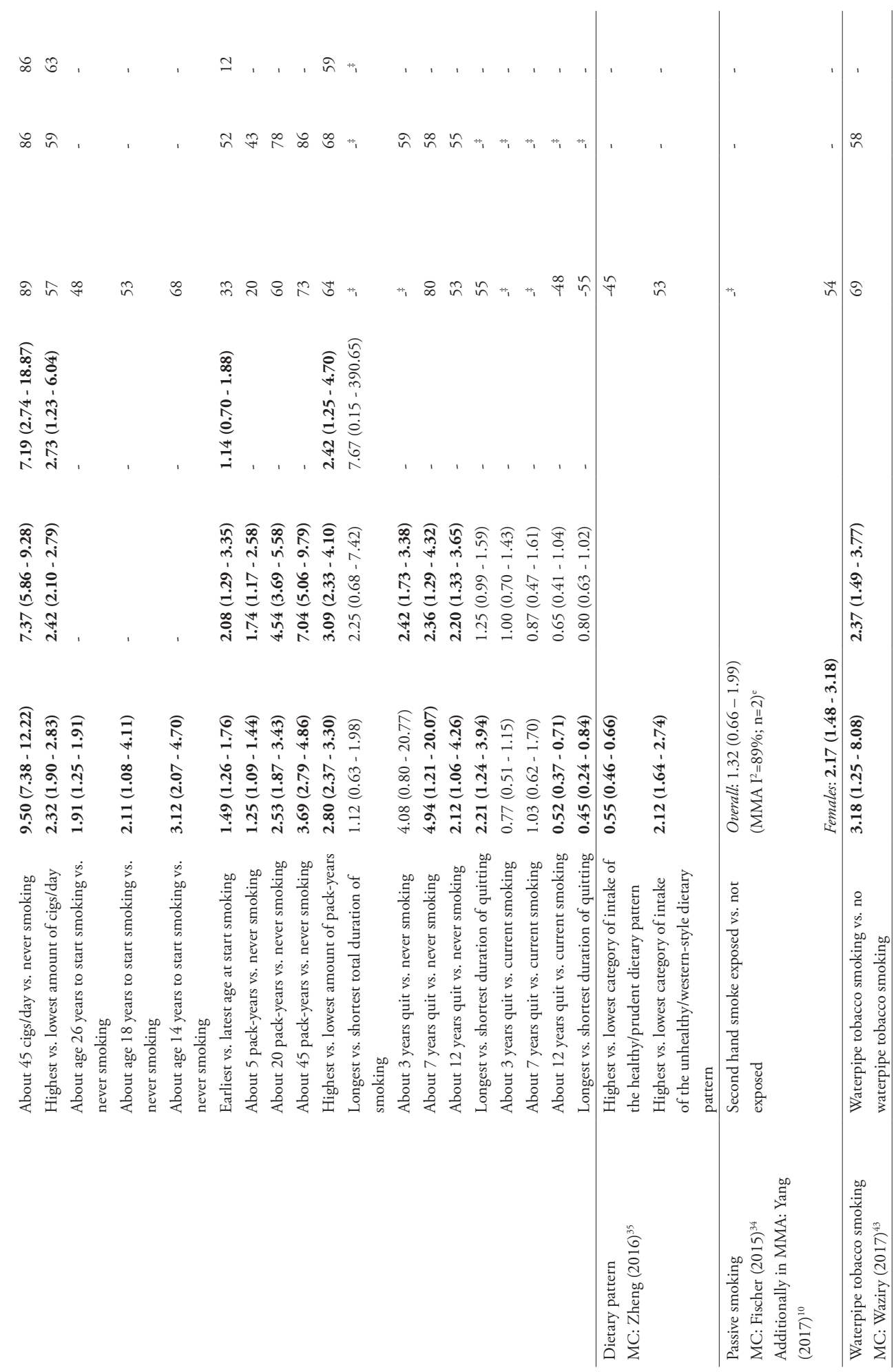


Chapter 3 | Genetic, lifestyle, and environmental risk factors of COPD development

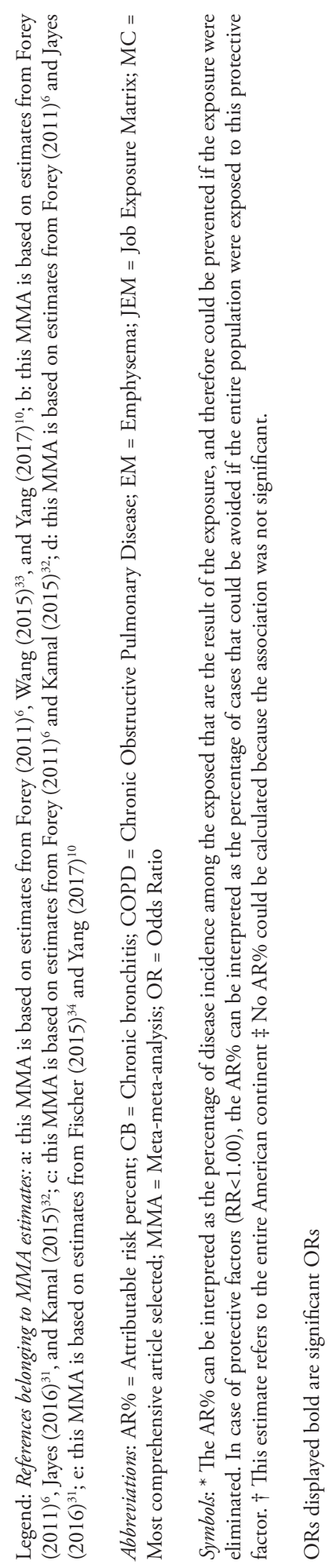


Chapter 3 | Genetic, lifestyle, and environmental risk factors of COPD development

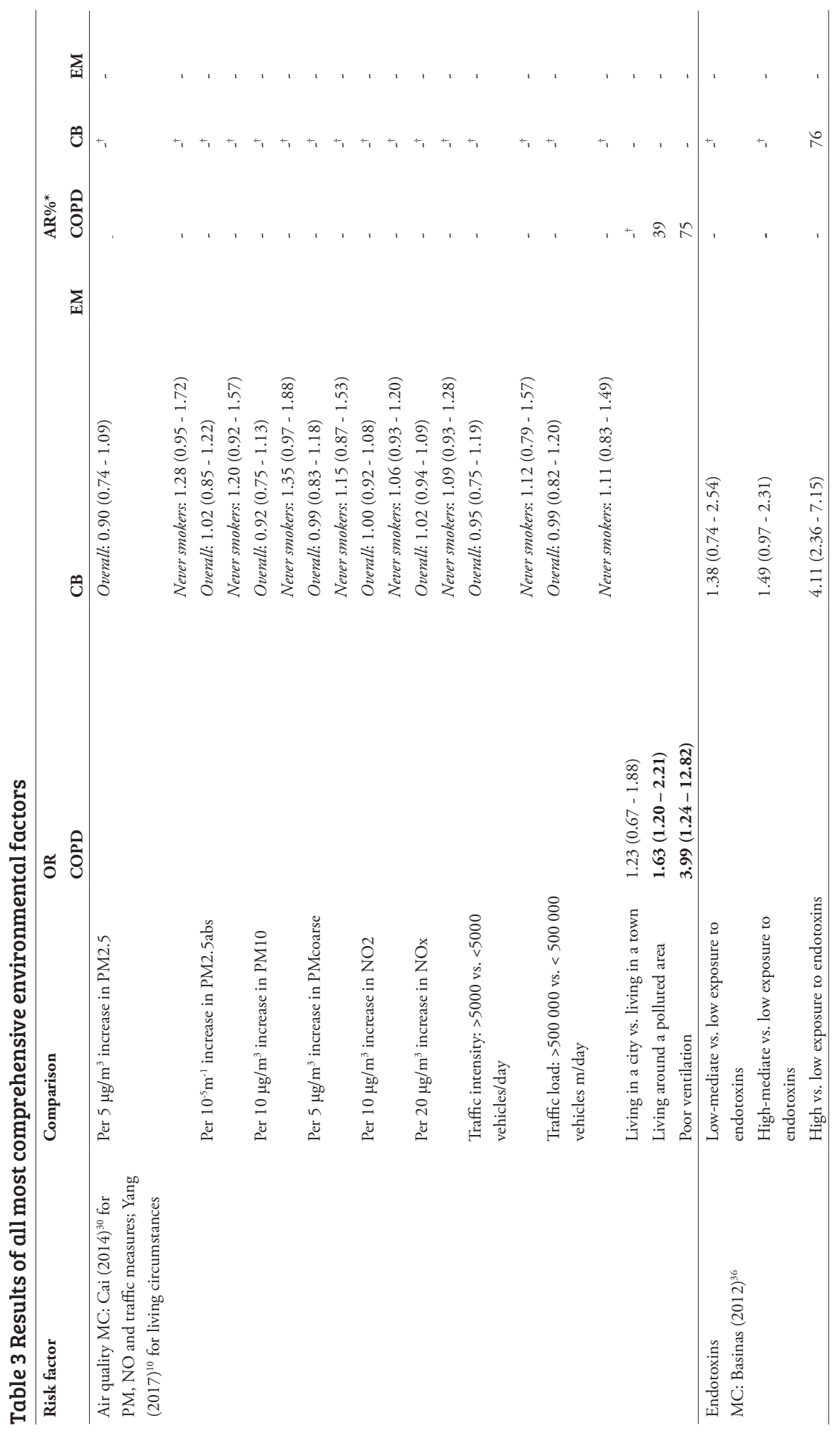


Chapter 3 | Genetic, lifestyle, and environmental risk factors of COPD development

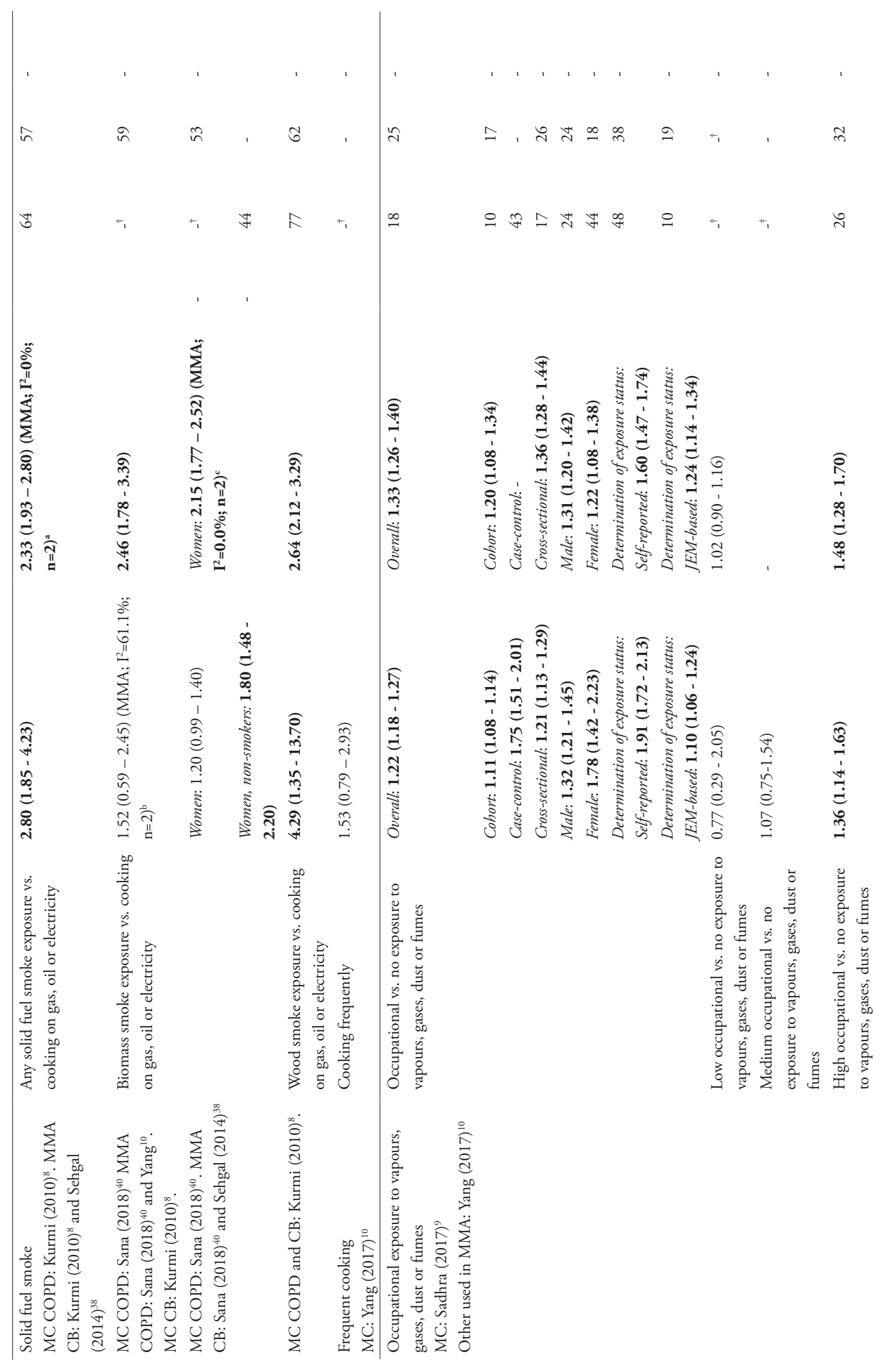


Chapter 3 | Genetic, lifestyle, and environmental risk factors of COPD development

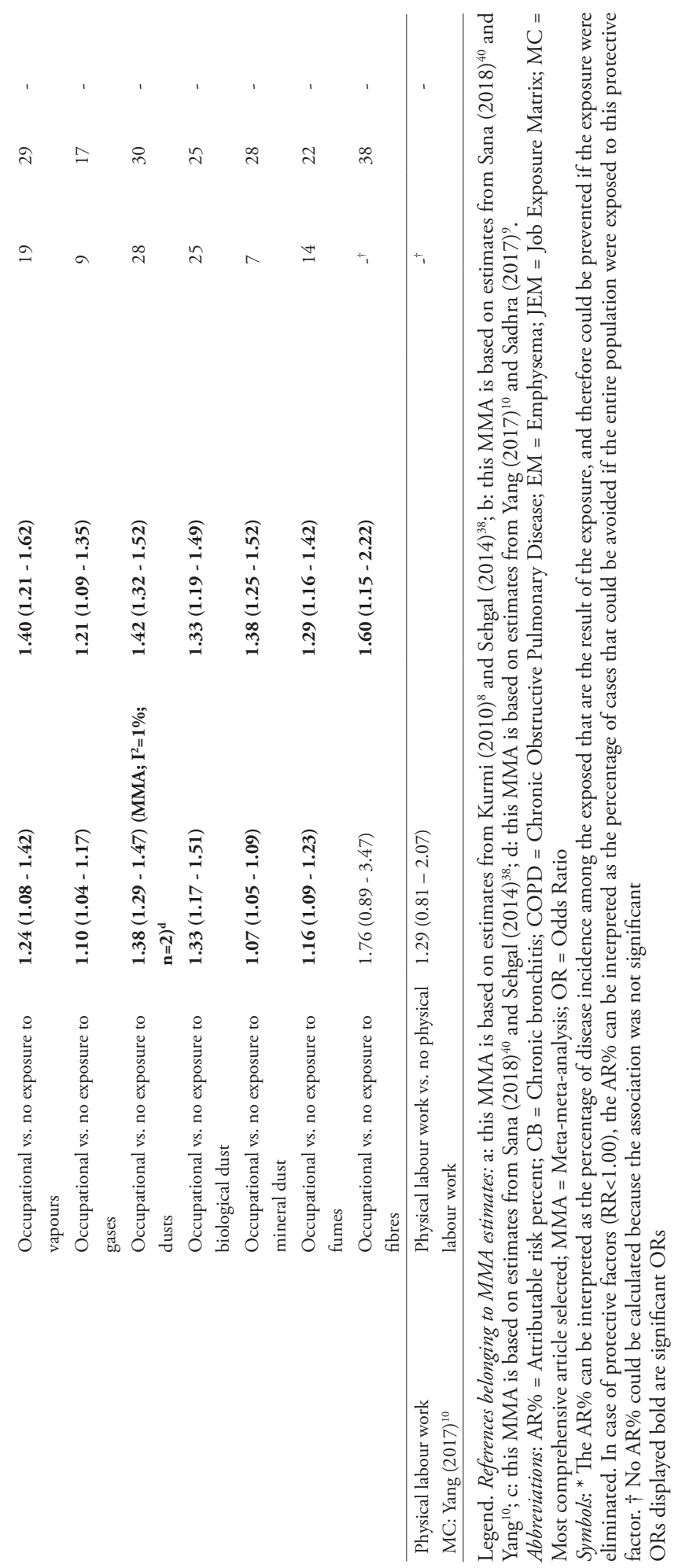




\section{Air quality}

Two publications ${ }^{10,30}$ studied different parameters of air quality. Living in a polluted area and poor housing ventilation increased COPD risk $\left(\mathrm{OR}_{\mathrm{COPD}}=1.63\right),\left(\mathrm{OR}_{\mathrm{COPD}}=3.99\right)$, as studied in Chinese populations. ${ }^{10}$

\section{Active smoking}

In total, five publications ${ }^{6,10,31-33}$ examined COPD risks associated with active smoking. Only one of those ${ }^{6}$ studied the risk of CB and EM. For several COPD comparisons, meta-meta-analyses could be performed. Compared to never or non-smoking, risks for all outcomes were significantly increased for ever smoking $\left(\mathrm{mOR}_{\mathrm{COPD}}=2.61, \mathrm{OR}_{\mathrm{CB}}=2.69\right.$ and $\left.\mathrm{OR}_{\mathrm{EM}}=4.51\right)$, current smoking $\left(\mathrm{mOR}_{\mathrm{COPD}}=3.51, \mathrm{OR}_{\mathrm{CB}}=3.41\right.$ and $\left.\mathrm{OR}_{\mathrm{EM}}=4.87\right)$, and former smoking $\left(\mathrm{mOR}_{\mathrm{COPD}},=2.44, \mathrm{OR}_{\mathrm{CB}}=1.63\right.$, and $\left.\mathrm{OR}_{\mathrm{EM}}=3.52\right)$.

Risks differed by study design, particularly for COPD. The risk for ever smoking was greatest in cohort studies, whereas for current and former smoking the risk was greatest in case-control studies. Similarly, the risks for current smoking for EM were highest in cross-sectional studies. Stratification by sex showed that for all comparisons and outcomes risks were higher for males than females. Finally, stratification by country showed that, with some exceptions, risks were greatest in North-American studies.

Forey et al. (2011) ${ }^{6}$ also studied other smoking-related parameters, such as intensity, duration, age at start smoking, and duration of quitting. In general, risks increased with increased intensity of smoking (all outcomes) and younger age at start smoking (COPD data only). Risks reduced with increased duration of quitting (data on COPD and CB only), although after 12 years of quitting the risk was still about a twofold increase compared to never smokers.

\section{Passive smoking}

The COPD risk for passive smoking was increased in females only $\left(\mathrm{OR}_{\mathrm{COPD}}=2.17\right)^{34}$, whereas the combined estimate was non-significant ${ }^{10,34}$.

\section{Dietary patterns}

High intake of an unhealthy or western diet (rich in red and processed meats, refined grains, sweets, desserts, and French fries) was associated with an increased risk $\left(\mathrm{OR}_{\mathrm{COPD}}=2.12\right)$ whereas high intake of a healthy or prudent diet (rich in vegetables, fruit, fish, and wholegrain) decreased the risk $\left(\mathrm{OR}_{\mathrm{COPD}}=0.55\right)^{35}$.

\section{Endotoxins}

Endotoxin exposure significantly increased $\mathrm{CB}$ risk at high exposures $\left(\mathrm{OR}_{\mathrm{CB}}=4.11\right)$, but 
not at low-mediate and high-mediate exposures ${ }^{36}$.

\section{Solid fuel smoke}

In total, six publications $s^{8,10,37-40}$ examined solid fuel smoke exposure and the COPD or CB. For COPD, Sana $(2018)^{40}$ was most comprehensive for biomass exposure and could be combined with Yang $(2017)^{10}$ in MMA for the most comprehensive estimate for both males and females combined. In addition, Kurmi $(2010)^{8}$ provided estimates for different solid fuels combined and wood burning exposure. All types of solid fuel smoke combined increased the risk of COPD with an $\mathrm{OR}=2.80$. Sub-estimates by type of fuel showed that particularly wood smoke increased the risk $(\mathrm{OR}=4.29)$ and no significant association for biomass smoke exposure.

For CB Kurmi $(2010)^{8}$ was most comprehensive, offering estimates for biomass and wood smoke exposure in both genders combined. In addition, Sana $(2018)^{40}$ and Sehgal $(2014)^{38}$ could be combined in MMA to obtain female-specific estimates. Overall, the risk of $\mathrm{CB}$ increased by over two-fold as a result of solid fuel smoke exposure $(\mathrm{OR}=$ 2.33), with similar estimates for biomass and wood-smoke exposure.

\section{Occupational exposure to vapors, gases, dusts or fumes}

In total, four publications $s^{9,10,41,42}$ reported on exposure to vapors, gases, dusts or fumes in relation to COPD or CB. Sadhra et al. $(2017)^{9}$ was most comprehensive for both outcomes. This publication was combined with Yang et al. (2017) ${ }^{10}$ for exposure to dusts specifically for COPD risk. Both COPD and $\mathrm{CB}$ risk were increased in the overall estimates $\left(\mathrm{OR}_{\mathrm{COPD}}=1.22\right.$ and $\left.\mathrm{OR}_{\mathrm{CB}}=1.33\right)$. The $\mathrm{COPD}$ risk was higher among females than males $\left(\mathrm{OR}_{\mathrm{COPD}}=1.78\right.$ and $\mathrm{OR}_{\mathrm{COPD}}=1.32$ respectively), whereas this was inversed for $\mathrm{CB}\left(\mathrm{OR}_{\mathrm{CB}}=1.22\right.$ and $\mathrm{OR}_{\mathrm{CB}}=1.31$ respectively). Stratified by study design, the risk was lower for cohort studies $\left(\mathrm{OR}_{\mathrm{COPD}}=1.11, \mathrm{OR}_{\mathrm{CB}}=1.20\right)$ than for case-control $\left(\mathrm{OR}_{\mathrm{COPD}}=1.75, \mathrm{OR}_{\mathrm{CB}}=\mathrm{N} / \mathrm{A}\right.$ cross-sectional studies $\left(\mathrm{OR}_{\mathrm{COPD}}=1.21, \mathrm{OR}_{\mathrm{CB}}=1.36\right)$. Furthermore, when stratified by level of exposure, the risks were only significant for high exposures $\left(\mathrm{OR}_{\mathrm{COPD}}=1.36, \mathrm{OR}_{\mathrm{CB}}=1.48\right)$, but not at low or medium level exposures. Finally, stratified by type of exposure, risks were significantly increased for both COPD and $\mathrm{CB}$ for vapors $\left(\mathrm{OR}_{\mathrm{COPD}}=1.24, \mathrm{OR}_{\mathrm{CB}}=1.40\right)$, gases $\left(\mathrm{OR}_{\mathrm{COPD}}=1.10, \mathrm{OR}_{\mathrm{CB}}=1.21\right)$, dusts $\left(\mathrm{mOR}_{\mathrm{COPD}}=1.38, \mathrm{OR}_{\mathrm{CB}}=1.42\right)$, biological dusts $\left(\mathrm{OR}_{\mathrm{COPD}}=1.33, \mathrm{OR}_{\mathrm{CB}}=1.33\right)$, mineral dusts $\left(\mathrm{OR}_{\mathrm{COPD}}=1.07, \mathrm{OR}_{\mathrm{CB}}=1.38\right)$, and fumes $\left(\mathrm{OR}_{\mathrm{COPD}}=1.16, \mathrm{OR}_{\mathrm{CB}}=1.29\right)$. For exposure to fibers, the risk was only significantly increased for $C B\left(O R_{C B}=1.60\right)$.

\section{Waterpipe tobacco smoking}

Waterpipe tobacco smoking increased the risk of both $\mathrm{COPD}$ and $\mathrm{CB}\left(\mathrm{OR}_{\mathrm{COPD}}=3.18\right.$ and $\mathrm{OR}_{\mathrm{CB}}=2.37 .{ }^{43}$ 


\section{Attributable risk percent}

The AR\% was calculated for each lifestyle and environmental/occupational factor (Tables 4 and 5). For COPD the AR\%/ ranged from $-55 \%$ (longest duration of quitting vs. current smoking) to $89 \%$ (smoking about 45 cigarettes per day vs. never smoking). For $\mathrm{CB}$ the $\mathrm{AR} \%$ ranged from $-35 \%$ (12 years quit smoking vs. current smoking) to $86 \%$ (smoking about 45 cigarettes per day vs. never smoking and smoking about 45 packyears vs. never smoking). Finally, for EM the AR\% ranged from $12 \%$ (earliest vs. latest age at start smoking) to $89 \%$ (current vs. never smoking in North American studies). Combining the modifiable factors of active smoking and diet, $87 \%$ of cases among current smokers who have poor dietary habits could be avoided with the adoption of an optimal lifestyle.

\section{Discussion}

To understand the origins of COPD, we consider it relevant to recognize that multiple determinants interact in development of COPD, each with varying degrees of importance. ${ }^{44}$ To our knowledge, this is the first systematic review and meta-metaanalysis of genetic, lifestyle, and environmental factors associated with COPD risk. It provides a comprehensive, reliable, and up-to-date summary of current knowledge at the highest level of evidence.

Exposure to toxic substances, such as those found in tobacco smoke, are well-known risk factors of COPD. In fact, smoking is one of the riskiest behaviors with regard to COPD. ${ }^{6,31-33}$ However, several environmental factors such as the use of solid fuels for cooking, also contribute to the risk of COPD. ${ }^{8,9,38}$ Polycyclic aromatic hydrocarbons (PAHs), amongst others, are present in cigarette smoke and (indoor) air pollution. ${ }^{45}$ They have been found to be related to the development of $\mathrm{COPD}^{46,47}$, although not consistently ${ }^{30}$. However, this may be due to methodological difficulties regarding measuring the extent of air pollution exposure. Highlighting the relevance of these exposures are the variants in genes involved in detoxification of PAHs, such as EPHX1. The extremely slow variants of the by EPHX1 encoded enzyme epoxide hydrolase 1, increased the COPD risk by $77 \%{ }^{17}$, which supports the idea that not only do higher exposures to these chemicals increase the risk of COPD, but also genetically determined metabolic limitations. Interestingly, and unexpectedly considering the importance of active smoking as a risk factor for COPD, our MMA results showed that passive smoking was not associated with COPD. In addition, we noted a lack of MAs on prenatal and childhood exposure to passive tobacco smoking in relation to COPD risk. It should be noted that a link is not unlikely given the results of previous research, 
which report poorer lung function and increased risk of chronic respiratory disease in offspring exposed to tobacco smoke prenatally or during childhood. ${ }^{48-50}$ The absence of high-level evidence on this topic is possibly due to methodological and ethical difficulty in collecting such data.

Variants in genes involved in addictive behaviors are also likely candidates to increase disease risk, as they may increase exposure to toxic substances. A great number of SNPs have been identified in the CHRNA3 and CHRNA5 genes ${ }^{51-53}$, encoding a nicotinic cholinergic receptor ${ }^{10}$. Variants in these genes may lead to an exaggerated pleasure in response to smoking and thereby increasing the chances of repeated exposure. ${ }^{54}$ Similarly, the MMP genes mediate a variety of biological functions including a role in the body's reward system, aiding in potentiating the rewarding effects of drugs. ${ }^{55,56}$ Our results show a significant association for MMP9 rs17576 and rs3918242, but not for any variants in MMP1, MMP3 or MMP12.

Chronic inflammation plays a critical role in the pathogenesis and progression of COPD. ${ }^{57}$ Many genes dysregulated by exposure to cigarette smoke are those related to oxidative stress and central components of inflammatory signaling pathways. ${ }^{58}$ One wellknown COPD-causing gene is the SERPINA1 gene, encoding $\alpha 1$-antitrypsin (AAT). AAT protects the alveoli from neutrophil elastase and other proteases. ${ }^{59,60}$ However, if this gene is mutated it may cause AAT-deficiency, which leads to emphysema and earlyonset or more severe forms of COPD, particularly in the presence of cigarette smoking or infection. ${ }^{61}$ Approximately one in 2500 people worldwide suffer from AAT-deficiency, occurring most frequently in Caucasians. ${ }^{62}$ The deficient $S$ and $\mathrm{Z}$ allele (slow and very slow enzyme activity respectively) led to an increased COPD risk of $\mathrm{OR}=3.26$ and $\mathrm{OR}=2.31$ respectively compared to the normal $\mathrm{M}$ allele. ${ }^{25,26}$ However, this association disappeared when limiting to smokers only. This may be the result of upregulation of other genes involved in the same pathway as a result of the frequent presence of cigarette smoke. ${ }^{63}$ Such an interaction was also seen in other gene variants, like TNF, and thus calls for particular attention towards smoking cessation interventions.

Dietary intake has also been associated with COPD risk as consumption of an unhealthy/western style diet is associated with an increased OR of 2.12 compared to a healthy/prudent dietary pattern. ${ }^{64}$ Literature suggests that antioxidants including certain omega-3 fatty acids, vitamins and minerals found in fruits and vegetables may mitigate the inflammatory effects of smoking. A recent review showed evidence for the positive influence of omega 3 fatty acids and vitamin E, which is at least partly attributable to their anti-inflammatory actions. ${ }^{65}$ However, this may be confounded by the association with other health behaviors. ${ }^{64}$ 
Several associations differed by ethnicity or sex, for example, the rs2280090 (T2) of the ADAM33 gene showed an increased risk among Europeans but decreased risk for Asians. Furthermore, several variants in the FOXO1 gene showed a decreased risk among female smokers only. ${ }^{51}$ An explanation for these ethnicity and sex-related differences may be due to different allele frequencies or exposure levels between populations or groups, (yet unknown) gene-environment interactions, linkage disequilibrium or remaining confounding factors. ${ }^{15}$

Unfortunately or fortunately, depending on one's perspective, is that a great number of cases could be prevented if prevalent behaviors such as smoking or poor dietary habits were eliminated. An unhealthy or Western-style diet is attributable for $53 \%$ of the cases. Even more substantial, $72 \%$ of cases among smokers could have been prevented by not smoking (tables 2 and 3). Furthermore gene-environment interactions may further help explain COPD etiology more precisely. Unfortunately, evidence on gene-environment interactions was often limited to stratifications by smoking status. COPD is a disease of circumstance, not every smoker will develop the disease and not every non-smoker will escape it. The likely cause is an interaction between different lifestyle, environmental and genetic factors. Although gene-environment interaction studies are becoming more prevalent, they are currently likely still limited due to methodological problems such as limited sample sizes, variant prevalence, and reproducibility of their findings. ${ }^{66}$ Nevertheless, they should remain a focus of future investigations.

\section{Limitations}

Our results are currently the most comprehensive available, yet they should be interpreted with caution. We urge the reader to critically appraise the results of each meta-analysis, when applying our results to their own purpose. Firstly, the quality of our results is dependent on the quality of the included meta-analyses. We judged their quality using the AMSTAR checklist, including all studies regardless of their score. These ratings showed that quality can be improved. For example, many studies did not do a comprehensive literature search, limiting to only one database and not searching grey literature, which may have introduced publication bias. ${ }^{67}$ Also, only a few studies provided a published 'a priori' design. Secondly, it is essential that the Meta-analyses sufficiently deal with the quality of their included studies, by both evaluating it and taking the result into consideration when formulating conclusions. Thirdly, not all studies reported risks by smoking status and primary studies had different baseline smoking exposures. As smoking is such a major risk factor for COPD, this information is critical when generalizing these results to the general population, for example in the use for gene-environment interaction analyses, pathway analyses, or the development of preventive strategies and genetic risk scores. Even more so, baseline smoking exposure 
may be particularly relevant when the gene is involved in smoking-related metabolism. Fourthly, we have not checked potential linkage disequilibrium between genetic variants found in different meta-analyses. If linkage disequilibrium is present, two significantly associated SNPs may actually be the effect of one single variant. Fifthly, as a result of the increased risk of a type II error in GWA studies, correction for multiple testing and therefore a very high statistical significance needs to be achieved for variants to be recognized as risk factors in GWA studies. However, as a result, some actual predictive variants may have been missed and it is possible that different variants may be identified in future studies. ${ }^{44}$ Finally, few meta-analyses could be combined in meta-meta-analysis due to great amount of overlap in primary studies between them, which would result in false precision and homogeneity if combined.$^{68}$ Even so, taken together, our results still represent an overview of the most comprehensive estimates currently available.

\section{Conclusion}

A large number of genetic, lifestyle, and environmental factors have been identified that each contribute to the development of COPD, either independently or through mutual interactions. Strongest genetic associations were found forADAM33 and the PI SZ genotype of SERPINA. Among the lifestyle factors, adopting a healthy diet, and avoiding active, passive and waterpipe smoking can result in great risk improvements. Based on our findings we suggest that future research should focus on gene-environment and gene-gene interactions.

The data collected in this overview lends itself to pathway analysis, which integrates interactions of genes and metabolites in specific biological processes. ${ }^{69}$ Using our data in such context will provide new insight into the pathway of COPD development, the relationships with other chronic diseases and improve disease outcome prediction models. $^{70}$

\section{Declaration of interests}

The authors declare no conflicting interests.

\section{Contributors}

All authors designed and approved of the research protocol. SM and KS did literature search, assessed eligibility of studies for inclusion, and extracted data. AW was consulted in case of disagreement. KS did the statistical analysis in consultation with SM and 
AW. SM and KS wrote the first draft of the article. All authors critically revised the manuscript for intellectual content, finally approved of the version to be published, and agree to be accountable for all aspects of the work in ensuring that questions related to the accuracy or integrity of any part of the work are appropriately investigated and resolved. 
Chapter 3 | Genetic, lifestyle, and environmental risk factors of COPD development

\section{References}

1. Guirguis-Blake JM, Senger CA, Webber EM, Mularski RA, Whitlock EP. Screening for chronic obstructive pulmonary disease: evidence report and systematic review for the US preventive services task force. Jama. 2016;315(13):1378-93.

2. Adeloye D, Chua S, Lee C, Basquill C, Papana A, Theodoratou E, et al. Global and regional estimates of COPD prevalence: Systematic review and meta-analysis. Journal of global health. 2015;5(2).

3. Alwan A. Global status report on noncommunicable diseases 2010: World Health Organization; 2011.

4. Raherison C, Girodet P. Epidemiology of COPD. European Respiratory Review. 2009;18(114):21321.

5. Postma DS, Bush A, van den Berge M. Risk factors and early origins of chronic obstructive pulmonary disease. The Lancet. 2015;385(9971):899-909.

6. Forey BA, Thornton AJ, Lee PN. Systematic review with meta-analysis of the epidemiological evidence relating smoking to COPD, chronic bronchitis and emphysema. BMC pulmonary medicine. 2011;11. PubMed PMID: WOS:000208592800036.

7. Bosse Y. Updates on the COPD gene list. International journal of chronic obstructive pulmonary disease. 2012;7:607-31. PubMed PMID: 23055711. Pubmed Central PMCID: PMC3459654. Epub 2012/10/12. eng.

8. Kurmi OP, Semple S, Simkhada P, Smith WCS, Ayres JG. COPD and chronic bronchitis risk of indoor air pollution from solid fuel: a systematic review and meta-analysis. Thorax. 2010 Mar;65(3):221-8. PubMed PMID: WOS:000276158700010.

9. Sadhra S, Kurmi OP, Sadhra SS, Lam KBH, Ayres JG. Occupational COPD and job exposure matrices: a systematic review and meta-analysis. International journal of chronic obstructive pulmonary disease. 2017;12:725-34. PubMed PMID: WOS:000394526600001.

10. Yang Y, Mao J, Ye Z, Li J, Zhao H, Liu Y. Risk factors of chronic obstructive pulmonary disease among adults in Chinese mainland: A systematic review and meta-analysis. Respiratory medicine. 2017;131:158-65.

11. Shea BJ, Grimshaw JM, Wells GA, Boers M, Andersson N, Hamel C, et al. Development of AMSTAR: a measurement tool to assess the methodological quality of systematic reviews. BMC medical research methodology. 2007;7(1):1.

12. Higgins JP, Thompson SG, Deeks JJ, Altman DG. Measuring inconsistency in meta-analyses. BMJ: British Medical Journal. 2003;327(7414):557.

13. Bouter LM, Zielhuis GA, Zeegers MP. Textbook of Epidemiology. 1 ed: Bohn Stafleu van Loghum; 2018. 288 p.

14. Kang SW, Kim SK, Chung JH, Jung HJ, Kim KI, Kim J, et al. Genetic Polymorphism of Angiotensin-Converting Enzyme and Chronic Obstructive Pulmonary Disease Risk: An Updated Meta-Analysis. BioMed research international. 2016;2016 (no pagination)(7636123). PubMed PMID: 613100538.

15. Zhou DC, Zhou CF, Toloo S, Shen T, Tong SL, Zhu QX. Association of a disintegrin and metalloprotease 33 (ADAM33) gene polymorphisms with the risk of COPD: an updated metaanalysis of 2,644 cases and 4,804 controls. Molecular biology reports. 2015 Feb;42(2):409-22. PubMed PMID: 25280544. Epub 2014/10/05. eng.

16. Wang CD, Chen N, Huang L, Wang JR, Chen ZY, Jiang YM, et al. Impact of CYP1A1 Polymorphisms on Susceptibility to Chronic Obstructive Pulmonary Disease: A Meta-Analysis. BioMed research international. 2015;2015 (no pagination)(942958). PubMed PMID: 605998816.

17. Li H, Fu WP, Hong ZH. Microsomal epoxide hydrolase gene polymorphisms and risk of chronic obstructive pulmonary disease: A comprehensive meta-analysis. Oncology letters. 2013 Mar;5(3):1022-30. PubMed PMID: 23426996. Pubmed Central PMCID: PMC3576314. Epub 2013/02/22. eng. 
18. Ding Z, Wang K, Li J, Tan Q, Tan W, Guo G. Association between Glutathione S-transferase Gene M1 and T1 Polymorphisms and Chronic Obstructive Pulmonary Disease Risk: A Meta-analysis. Clinical genetics. 2018.

19. Xue H, Su J, Sun K, Xie W, Wang H. Glutathione S-transferase M1 and T1 gene polymorphism and COPD risk in smokers: an updated analysis. Molecular biology reports. 2012 Apr;39(4):503342. PubMed PMID: 22160572. Epub 2011/12/14. eng.

20. Zhou H, Ying X, Liu Y, Ye S, Yan J, Li Y. Genetic polymorphism of heme oxygenase 1 promoter in the occurrence and severity of chronic obstructive pulmonary disease: a meta-analysis. Journal of cellular and molecular medicine. 2017;21(5):894-903.

21. Ji G-Y, Tang X-Y, Wu S-Q, Wang Y, Liu Q-Q, Wu J-C, et al. Association between the IL-6 rs1800796 (-572C/G) polymorphism and chronic obstructive pulmonary disease risk: a metaanalysis. International journal of clinical and experimental medicine. 2017;10(7):10375-81.

22. Chen L, Shen Y, Liu L, Li X, Wang T, Wen F. Interleukin-13 -1112 C/T promoter polymorphism confers risk for COPD: a meta-analysis. PloS one. 2013;8(7):e68222. PubMed PMID: 23874547. Pubmed Central PMCID: PMC3706578. Epub 2013/07/23. eng.

23. Duan L, Liang R, Wang Z, Lei L, Jin L, Shen J, et al. Lack of association between the G+2044A polymorphism of interleukin-13 gene and chronic obstructive pulmonary disease: a metaanalysis. Molecular biology reports. 2014 Sep;41(9):6297-303. PubMed PMID: 24990692. Epub 2014/07/06. eng.

24. Xie ZK, Huang QP, Huang J, Xie ZF. Association between the IL1B, IL1RN polymorphisms and COPD risk: a meta-analysis. Scientific reports. 2014;4:6202. PubMed PMID: 606447192.

25. Hersh C, Dahl M, Ly N, Berkey C, Nordestgaard B, Silverman E. Chronic obstructive pulmonary disease in $\alpha 1$-antitrypsin PI MZ heterozygotes: a meta-analysis. Thorax. 2004;59(10):843-9.

26. Dahl M, Hersh C, Ly N, Berkey C, Silverman E, Nordestgaard B. The protease inhibitor PI* S allele and COPD: a meta-analysis. European Respiratory Journal. 2005;26(1):67-76.

27. Ma T, Liu X, Liu Z. Functional polymorphisms in surfactant protein genes and chronic obstructive pulmonary disease risk: a meta-analysis. 2. 2013 Dec;17(12):910-7. PubMed PMID: 24093802. Epub 2013/10/08. eng.

28. Zhang L, Gu H, Gu Y, Zeng X. Association between TNF-alpha -308 G/A polymorphism and COPD susceptibility: a meta-analysis update. International journal of chronic obstructive pulmonary disease. 2016;11:1367-79. PubMed PMID: 27382273. Pubmed Central PMCID: PMC4922767. Epub 2016/07/07. eng.

29. Cui K, Ge XY, Ma HL. Association of -238G/A and -863C/A polymorphisms in the TNF-alpha gene with chronic obstructive pulmonary disease based on a meta-analysis. Genetics and Molecular Research. 201324 Oct;12(4):4981-9. PubMed PMID: 370219873.

30. Cai YT, Schikowski T, Adam M, Buschka A, Carsin AE, Jacquemin B, et al. Cross-sectional associations between air pollution and chronic bronchitis: an ESCAPE meta-analysis across five cohorts. Thorax. 2014 Nov;69(11):1005-14. PubMed PMID: WOS:000344101700009.

31. Jayes L, Haslam PL, Gratziou CG, Powell P, Britton J, Vardavas C, et al. SmokeHaz Systematic Reviews and Meta-analyses of the Effects of Smoking on Respiratory Health. Chest. 2016 Jul;150(1):164-79. PubMed PMID: WOS:000379220000029.

32. Kamal R, Srivastava AK, Kesavachandran CN. Meta-analysis approach to study the prevalence of chronic obstructive pulmonary disease among current, former and non-smokers. 2015.

33. Wang BY, Xiao D, Wang C. Smoking and chronic obstructive pulmonary disease in Chinese population: a meta-analysis. Clinical Respiratory Journal. 2015 Apr;9(2):165-75. PubMed PMID: WOS:000352561500005.

34. Fischer F, Kraemer A. Meta-analysis of the association between second-hand smoke exposure and ischaemic heart diseases, COPD and stroke. BMC public health. 2015 Dec;15. PubMed PMID: WOS:000365477400003.

35. Zheng PF, Shu L, Si CJ, Zhang XY, Yu XL, Gao W. Dietary Patterns and Chronic Obstructive Pulmonary Disease: A Meta-analysis. Copd-Journal of Chronic Obstructive Pulmonary Disease. 
Chapter 3 | Genetic, lifestyle, and environmental risk factors of COPD development

2016;13(4):515-22. PubMed PMID: WOS:000381019000015.

36. Basinas I, Schlunssen V, Heederik D, Sigsgaard T, Smit LAM, Samadi S, et al. Sensitisation to common allergens and respiratory symptoms in endotoxin exposed workers: a pooled analysis. Occupational and environmental medicine. 2012 Feb;69(2):99-106. PubMed PMID: WOS:000299307700004.

37. Po JYT, FitzGerald JM, Carlsten C. Respiratory disease associated with solid biomass fuel exposure in rural women and children: systematic review and meta-analysis. Thorax. 2011 Mar;66(3):232-9. PubMed PMID: WOS:000287478700013.

38. Sehgal M, Rizwan SA, Krishnan A. Disease burden due to biomass cooking-fuel-related household air pollution among women in India. Global Health Action. 2014;7. PubMed PMID: WOS:000345021900001.

39. Hu G, Zhou Y, Tian J, Yao W, Li J, Li B, et al. Risk of COPD from exposure to biomass smoke: a metaanalysis. Chest Journal. 2010;138(1):20-31.

40. Sana A, Somda SM, Meda N, Bouland C. Chronic obstructive pulmonary disease associated with biomass fuel use in women: a systematic review and meta-analysis. BMJ open respiratory research. 2018;5(1):e000246.

41. Alif SM, Dharmage SC, Bowatte G, Karahalios A, Benke G, Dennekamp M, et al. Occupational exposure and risk of chronic obstructive pulmonary disease: a systematic review and metaanalysis. Expert review of respiratory medicine. 2016 Aug;10(8):861-72. PubMed PMID: WOS:000381048000004.

42. Ryu JY, Sunwoo YE, Lee SY, Lee CK, Kim JH, Lee JT, et al. Chronic Obstructive Pulmonary Disease (COPD) and Vapors, Gases, Dusts, or Fumes (VGDF): A Meta-analysis. CopdJournal of Chronic Obstructive Pulmonary Disease. 2015 Aug;12(4):374-80. PubMed PMID: WOS:000359849000004.

43. Waziry R, Jawad M, Ballout RA, Al Akel M, Akl EA. The effects of waterpipe tobacco smoking on health outcomes: an updated systematic review and meta-analysis. International journal of epidemiology. 2017;46(1):32-43.

44. Ortega VE. Picking the Right Fruit: Intersecting COPD GWAS Discoveries with Epigenetics. American journal of respiratory and critical care medicine. 2018 (ja).

45. Ewa B, Danuta M-Š. Polycyclic aromatic hydrocarbons and PAH-related DNA adducts. Journal of applied genetics. 2017;58(3):321-30.

46. Vibhuti A, Arif E, Mishra A, Deepak D, Singh B, Rahman I, et al. CYP1A1, CYP1A2 and CYBA gene polymorphisms associated with oxidative stress in COPD. Clinica chimica acta; international journal of clinical chemistry. 2010 Apr 02;411(7-8):474-80. PubMed PMID: 20080081. Epub 2010/01/19. eng.

47. Srogi K. Monitoring of environmental exposure to polycyclic aromatic hydrocarbons: a review. Environmental Chemistry Letters. 2007;5(4):169-95.

48. Svanes C, Omenaas E, Jarvis D, Chinn S, Gulsvik A, Burney P. Parental smoking in childhood and adult obstructive lung disease: results from the European Community Respiratory Health Survey. Thorax. 2004;59(4):295-302.

49. Drummond D, Baravalle-Einaudi M, Lezmi G, Vibhushan S, Franco-Montoya M-L, Hadchouel A, et al. Combined effects of in utero and adolescent tobacco smoke exposure on lung function in C57Bl/6J mice. Environmental health perspectives. 2017;125(3):392.

50. Perret JL, Walters H, Johns D, Gurrin L, Burgess J, Lowe A, et al. Mother's smoking and complex lung function of offspring in middle age: A cohort study from childhood. Respirology (Carlton, Vic). 2016;21(5):911-9.

51. Hardin M, Cho MH, Sharma SS, Glass K, Castaldi PJ, McDonald ML, et al. Sex-based Genetic Association Study Identifies CELSR1 as a Possible COPD Risk Locus Among Women. American journal of respiratory cell and molecular biology. 2016 Nov 17. PubMed PMID: 27854507. Epub 2016/11/18. eng.

52. Cui K, Ge X, Ma H. Four SNPs in the CHRNA3/5 alpha-neuronal nicotinic acetylcholine receptor 
subunit locus are associated with COPD risk based on meta-analyses. PloS one. 2014;9(7):e102324. PubMed PMID: 25051068. Pubmed Central PMCID: PMC4106784. Epub 2014/07/23. eng.

53. Cho MH, McDonald ML, Zhou X, Mattheisen M, Castaldi PJ, Hersh CP, et al. Risk loci for chronic obstructive pulmonary disease: a genome-wide association study and meta-analysis. The Lancet Respiratory medicine. 2014 Mar;2(3):214-25. PubMed PMID: 24621683. Pubmed Central PMCID: PMC4176924. Epub 2014/03/14. eng.

54. Sherva R, Wilhelmsen K, Pomerleau CS, Chasse SA, Rice JP, Snedecor SM, et al. Association of a single nucleotide polymorphism in neuronal acetylcholine receptor subunit alpha 5 (CHRNA5) with smoking status and with 'pleasurable buzzduring early experimentation with smoking. Addiction. 2008;103(9):1544-52.

55. Mizoguchi H, Yamada K, Mouri A, Niwa M, Mizuno T, Noda Y, et al. Role of matrix metalloproteinase and tissue inhibitor of MMP in methamphetamine-induced behavioral sensitization and reward: implications for dopamine receptor down-regulation and dopamine release. Journal of neurochemistry. 2007;102(5):1548-60.

56. Yamada K. Pro-addictive and anti-addictive factors for drug dependence. 2008.

57. Yao H, Rahman I. Current concepts on the role of inflammation in COPD and lung cancer. Current opinion in pharmacology. 2009;9(4):375-83.

58. Shaykhiev R, Crystal RG. Early events in the pathogenesis of chronic obstructive pulmonary disease. Smoking-induced reprogramming of airway epithelial basal progenitor cells. Annals of the American Thoracic Society. 2014;11(Supplement 5):S252-S8.

59. Little J, Higgins JP, Ioannidis JP, Moher D, Gagnon F, Von Elm E, et al. STrengthening the REporting of Genetic Association Studies (STREGA): an extension of the STROBE statement. Human genetics. 2009;125(2):131-51.

60. Gettins PG. Serpin structure, mechanism, and function. Chemical reviews. 2002;102(12):4751804.

61. Stoller JK, Aboussouan LS. $\alpha 1$-antitrypsin deficiency. The Lancet. 2005;365(9478):2225-36.

62. Hazari YM, Bashir A, Habib M, Bashir S, Habib H, Qasim MA, et al. Alpha-1-antitrypsin deficiency: Genetic variations, clinical manifestations and therapeutic interventions. Mutation Research/Reviews in Mutation Research. 2017;773:14-25.

63. Gunes A, Ozbey G, Vural EH, Uluoglu C, Scordo MG, Zengil H, et al. Influence of genetic polymorphisms, smoking, gender and age on CYP1A2 activity in a Turkish population. 2009.

64. Varraso R, Chiuve S, Fung T, Barr G, Hu F, Willett W, et al. Prospective cohort study of the alternate healthy eating index-2010 and risk of chronic obstructive pulmonary disease in US women and men.

65. de Boer A, van de Worp WR, Hageman GJ, Bast A. The effect of dietary components on inflammatory lung diseases-a literature review. International Journal of Food Sciences and Nutrition. 2017:1-17.

66. Hüls A, Ickstadt K, Schikowski T, Krämer U. Detection of gene-environment interactions in the presence of linkage disequilibrium and noise by using genetic risk scores with internal weights from elastic net regression. BMC genetics. 2017;18(1):55.

67. Hopewell S, McDonald S, Clarke MJ, Egger M. Grey literature in meta-analyses of randomized trials of health care interventions. The Cochrane Library. 2007.

68. Tramèr MR, Reynolds DJM, Moore RA, McQuay HJ. Impact of covert duplicate publication on meta-analysis: a case study. BMJ (Clinical research ed). 1997;315(7109):635-40.

69. Kutmon M, Lotia S, Evelo CT, Pico AR. WikiPathways App for Cytoscape: Making biological pathways amenable to network analysis and visualization. F1000Research. 2014;3.

70. Kao PY, Leung KH, Chan LW, Yip SP, Yap MK. Pathway analysis of complex diseases for GWAS, extending to consider rare variants, multi-omics and interactions. Biochimica et Biophysica Acta (BBA)-General Subjects. 2016.

71. Aierken H, Wang J, Wushouer Q, Shayhidin E, Hu X, Syed I, et al. Polymorphisms of the ADAM33 gene and chronic obstructive pulmonary disease risk: A meta-analysis. Clinical Respiratory Journal. 2014 January;8(1):108-15. PubMed PMID: 52863038. 
Chapter 3 | Genetic, lifestyle, and environmental risk factors of COPD development

72. An L, Xia H, Zhou P, Hua L. Exploration of association between EPHX1 and chronic obstructive pulmonary disease on the basis of combined data mining. Genetics and Molecular Research. 2016 26 Apr;15 (2) (no pagination)(15028639). PubMed PMID: 610079535.

73. Brogger J, Steen VM, Eiken HG, Gulsvik A, Bakke P. Genetic association between COPD and polymorphisms in TNF, ADRB2 and EPHX1. European Respiratory Journal. 2006 April;27(4):682-8. PubMed PMID: 44914189.

74. Castaldi PJ, Cho MH, Cohn M, Langerman F, Moran S, Tarragona N, et al. The COPD genetic association compendium: a comprehensive online database of COPD genetic associations. Human molecular genetics. 2010 Feb 01;19(3):526-34. PubMed PMID: 19933216. Pubmed Central PMCID: PMC2798725. Epub 2009/11/26. eng.

75. Chen H, Zhang L, He Z, Zhong X, Zhang J, Li M, et al. Vitamin D binding protein gene polymorphisms and chronic obstructive pulmonary disease: A meta-analysis. Journal of thoracic disease. 2015;7(8):1423-40. PubMed PMID: 605857205.

76. Chen L, Wang T, Liu L, Shen Y, Wan C, Wen F. Matrix Metalloproteinase-9 -1562C/T Promoter Polymorphism Confers Risk for COPD: A Meta-Analysis. PloS one. 201328 Mar;8 (3) (no pagination)(e60523). PubMed PMID: 368612235.

77. Cho MH, Boutaoui N, Klanderman BJ, Sylvia JS, Ziniti JP, Hersh CP, et al. Variants in FAM13A are associated with chronic obstructive pulmonary disease. Nature genetics. 2010;42(3):200.

78. Cho MH, Castaldi PJ, Wan ES, Siedlinski M, Hersh CP, Demeo DL, et al. A genome-wide association study of COPD identifies a susceptibility locus on chromosome 19q13. Human molecular genetics. 2012 February;21(4):947-57. PubMed PMID: 364163854.

79. Cui K, Ge XY, Ma HL. Association of the TNF-alpha +489 G/A polymorphism with chronic obstructive pulmonary disease risk in Asians: Meta-analysis. Genetics and Molecular Research. 201518 May;14(2):5210-20. PubMed PMID: 604425700.

80. Du Y, Xue Y, Xiao W. Association of IREB2 Gene rs2568494 Polymorphism with Risk of Chronic Obstructive Pulmonary Disease: A Meta-Analysis. Medical science monitor : international medical journal of experimental and clinical research. 2016 Jan 18;22:177-82. PubMed PMID: 26775557. Pubmed Central PMCID: PMC4723059. Epub 2016/01/19. eng.

81. Van Durme YMTA, Eijgelsheim M, Joos GF, Hofman A, Uitterlinden AG, Brusselle GG, et al. Hedgehog-interacting protein is a COPD susceptibility gene: The Rotterdam study. European Respiratory Journal. 2010 July;36(1):89-95. PubMed PMID: 359181015.

82. Gong Y, Fan L, Wan H, Shi Y, Shi G, Feng Y, et al. Lack of association between the TGF-beta(1) gene and development of COPD in Asians: a case-control study and meta-analysis. Lung. 2011 Jun;189(3):213-23. PubMed PMID: 21556788. Epub 2011/05/11. eng.

83. Hu G, Shi Z, Hu J, Zou G, Peng G, Ran P. Association between polymorphisms of microsomal epoxide hydrolase and COPD: results from meta-analyses. Respirology (Carlton, Vic). 2008 Nov;13(6):837-50. PubMed PMID: 18811882. Epub 2008/09/25. eng.

84. Hu G, Yao W, Zhou Y, Hu J, Shi Z, Li B, et al. Meta- and pooled analyses of the effect of glutathione S-transferase M1 and T1 deficiency on chronic obstructive pulmonary disease. The international journal of tuberculosis and lung disease : the official journal of the International Union against Tuberculosis and Lung Disease. 2008 Dec;12(12):1474-81. PubMed PMID: 19017460. Epub 2008/11/20. eng.

85. Hobbs BD, Parker MM, Chen H, Lao T, Hardin M, Qiao D, et al. Exome array analysis identifies a common variant in IL27 associated with chronic obstructive pulmonary disease. American journal of respiratory and critical care medicine. 2016;194(1):48-57.

86. Hobbs BD, de Jong K, Lamontagne M, Bossé Y, Shrine N, Artigas MS, et al. Genetic loci associated with chronic obstructive pulmonary disease overlap with loci for lung function and pulmonary fibrosis. Nature genetics. 2017;49(3):426-32.

87. Jiang S, Yang ZH, Chen YY, He Z, Zhou Y, Gao Y, et al. MMP-9 genetic polymorphism may confer susceptibility to COPD. Genetics and molecular research : GMR. 2016 Apr 25;15(2). PubMed PMID: 27173221. Epub 2016/05/14. eng. 
88. Lambrechts D, Buysschaert I, Zanen P, Coolen J, Lays N, Cuppens H, et al. The 15q24/25 susceptibility variant for lung cancer and chronic obstructive pulmonary disease is associated with emphysema. American journal of respiratory and critical care medicine. 2010 Mar 01;181(5):48693. PubMed PMID: 20007924. Epub 2009/12/17. eng.

89. Lee J, Nordestgaard BG, Dahl M. EPHX1 polymorphisms, COPD and asthma in 47,000 individuals and in meta-analysis. The European respiratory journal. 2011 Jan;37(1):18-25. PubMed PMID: 20516053. Epub 2010/06/03. eng.

90. Li DD, Guo SJ, Jia LQ, Wen FQ. Association of a disintegrin and metalloproteinase 33 (ADAM33) gene polymorphisms with chronic obstructive pulmonary disease in the Chinese population: A meta-analysis. Genetics and Molecular Research. 201425 Aug;13(3):6391-7. PubMed PMID: 373853877.

91. Li W, Lan F, Yan F, Shen H. Angiotensin-converting enzyme I/D polymorphism is associated with COPD risk in Asian population: evidence from a meta-analysis. Copd. 2013 Feb;10(1):35-9. PubMed PMID: 23272666. Epub 2013/01/01. eng.

92. Li X, Wei N, Wu Z, Qi Z, Zhang X, Chen Q, et al. The D/I polymorphism in the angiotensinconverting enzyme gene and chronic obstructive pulmonary disease risk: a meta-analysis. Copd. 2012 Aug;9(5):485-91. PubMed PMID: 22876865. Epub 2012/08/11. eng.

93. Liao N, Zhao H, Chen M-L, Z-F X. Association between the TGF- $\beta 1$ polymorphisms and chronic obstructive pulmonary disease: a meta-analysis. Biosci Rep. 2017;37(4).

94. Nedeljkovic I, Terzikhan N, Vonk JM, van der Plaat DA, Lahousse L, van Diemen CC, et al. A genome-wide linkage study for chronic obstructive pulmonary disease in a Dutch genetic isolate identifies novel rare candidate variants. Frontiers in genetics. 2018;9:133.

95. Nielsen AO, Jensen CS, Arredouani MS, Dahl R, Dahl M. Variants of the ADRB2 Gene in COPD: Systematic Review and Meta-Analyses of Disease Risk and Treatment Response. COPD: Journal of Chronic Obstructive Pulmonary Disease. 2017:1-10.

96. Niu LM, Liang Y, Xu M, Zhang YY, Zhang Y, He B. Effect of polymorphisms in the beta2adrenergic receptor on the susceptibility and pulmonary function of patients with chronic obstructive pulmonary disease: a meta analysis. Chinese medical journal. 2012 Jun;125(12):22138. PubMed PMID: 22884155. Epub 2012/08/14. eng.

97. Smolonska J, Wijmenga C, Postma DS, Boezen HM. Erratum: Meta-analyses on suspected chronic obstructive pulmonary disease genes: a summary of 20 years' research. American journal of respiratory and critical care medicine. 2010 Apr 01;181(7):765. PubMed PMID: 20355267. Epub 2010/04/01. eng.

98. Smolonska J, Wijmenga C, Postma DS, Boezen HM. Meta-analyses on suspected chronic obstructive pulmonary disease genes: A summary of 20 years' research. American journal of respiratory and critical care medicine. 200901 Oct;180(7):618-31. PubMed PMID: 355337572.

99. Tian DB, Cai SX, Pan GC, Zhang YF, Xiao JH. Association between the rs3795879 G/A polymorphism of the SERPINE2 gene and chronic obstructive pulmonary disease: A meta-analysis. Genetics and Molecular Research. 201514 Jul;14(3):7920-8. PubMed PMID: 605193568.

100. Wain LV, Shrine N, Artigas MS, Erzurumluoglu AM, Noyvert B, Bossini-Castillo L, et al. Genomewide association analyses for lung function and chronic obstructive pulmonary disease identify new loci and potential druggable targets. Nature genetics. 2017;49(3):416.

101. Wang YS, Liu L, Xu XY, Wang JL, Zhou H, Li TL, et al. Association of interleukin-1beta -511C/ $\mathrm{T}$ promoter polymorphism with COPD risk: a meta-analysis. Genetics and molecular research : GMR. 2015 May 04;14(2):4477-84. PubMed PMID: 25966220. Epub 2015/05/13. eng.

102. Wang YL, Kong H, Xie WP, Wang H. Association of vitamin D-binding protein variants with chronic obstructive pulmonary disease: a meta-analysis. Genetics and molecular research : GMR. 2015 Sep 09;14(3):10774-85. PubMed PMID: 26400306. Epub 2015/09/25. eng.

103. Wang W, Li P, Chen Y, Yang J. Association between beta2-Adrenergic Receptor-16Arg/Gly Gene Polymorphism and Chronic Obstructive Pulmonary Disease Risk:Systematic Review and MetaAnalysis. Iranian journal of public health. 2014 Jul;43(7):877-88. PubMed PMID: 25909056. 
Chapter 3 | Genetic, lifestyle, and environmental risk factors of COPD development

Pubmed Central PMCID: PMC4401053. Epub 2015/04/25. eng.

104. Xiao M, Guo L, Wang T, Zhu T, Jia L, Chen L, et al. Interleukin-1B-31T/C promoter polymorphism and chronic obstructive pulmonary disease risk: a meta-analysis. Archives of medical science: AMS. 2014 Jun 29;10(3):434-8. PubMed PMID: 25097571. Pubmed Central PMCID: PMC4107249. Epub 2014/08/07. eng.

105. Xiao M, Wang T, Zhu T, Wen F. Dual role of vitamin D-binding protein $1 \mathrm{~F}$ allele in chronic obstructive pulmonary disease susceptibility: a meta-analysis. Genetics and molecular research : GMR. 2015 Apr 17;14(2):3534-40. PubMed PMID: 25966120. Epub 2015/05/13. eng.

106. Xie X, Zhang Y, Ke R, Wang G, Wang S, Hussain T, et al. Vitamin D-binding protein gene polymorphisms and chronic obstructive pulmonary disease susceptibility: A meta-analysis. Biomedical reports. 2015 Mar;3(2):183-8. PubMed PMID: 25798246. Pubmed Central PMCID: PMC4360878. Epub 2015/03/24. eng.

107. Xie XM, Ke R, Zhang YH, Wang GZ, Zhu YT, Hussian T, et al. Interleukin-6 gene $-174 \mathrm{G}>\mathrm{C}$ polymorphism and chronic obstructive pulmonary disease risk: a meta-analysis. Genetics and molecular research : GMR. 2015 Jul 28;14(3):8516-25. PubMed PMID: 26345781. Epub 2015/09/09. eng.

108. Yan F, Chen C, Jing J, Li W, Shen H, Wang X. Association between polymorphism of glutathione S-transferase P1 and chronic obstructive pulmonary disease: a meta-analysis. Respiratory medicine. 2010 Apr;104(4):473-80. PubMed PMID: 20117922. Epub 2010/02/02. eng.

109. Yang L, Li X, Tong X, Fan H. Association between glutathione S-transferase P1 Ile (105) Val gene polymorphism and chronic obstructive pulmonary disease: A meta-analysis based on seventeen case-control studies. Meta gene. 2015 December 01;6:59-64. PubMed PMID: 606078302.

110. Zhang J, Summah H, Zhu YG, Qu JM. Nicotinic acetylcholine receptor variants associated with susceptibility to chronic obstructive pulmonary disease: a meta-analysis. Respiratory research. 2011 Dec 17;12:158. PubMed PMID: 22176972. Pubmed Central PMCID: PMC3283485. Epub 2011/12/20. eng.

111. Zhang L, Chang WW, Ding H, Su H, Wang HY. Transforming growth factor-beta1 polymorphisms and chronic obstructive pulmonary disease: A meta-analysis. International Journal of Tuberculosis and Lung Disease. 2011 October;15(10):1301-7. PubMed PMID: 362631041.

112. Zhang R, Li H, Zhao H, Chen W, Cheng D. Polymorphisms in a disintegrin and metalloprotease 33 gene and the risk of chronic obstructive pulmonary disease: A meta-analysis. Respirology (Carlton, Vic). 2014 April;19(3):312-20. PubMed PMID: 52966008.

113. Zhou H, Wu Y, Jin Y, Zhou J, Zhang C, Che L, et al. Genetic polymorphism of matrix metalloproteinase family and chronic obstructive pulmonary disease susceptibility: a meta-analysis. Scientific reports. 2013;3:2818. PubMed PMID: 563072343. 


\section{Supplementary files}

\section{Supplementary text 1 - Full search strategy}

Genetic factors

Pubmed: (pulmonary disease, chronic obstructive[MeSH Terms] OR ("pulmonary" [All Fields] AND "disease"[All Fields] AND "chronic"[All Fields] AND "obstructive"[All Fields]) OR "chronic obstructive pulmonary disease"[All Fields] OR "copd"[All Fields] OR pulmonary emphysema[MeSH Terms] OR "emphysema" [All Fields] OR "pulmonary emphysema" [All Fields] OR "emphysema”[MeSH Terms] OR bronchitis, chronic[MeSH Terms] OR "bronchitis"[All Fields] OR "chronic bronchitis" [All Fields] OR lung diseases, obstructive[MeSH Terms] OR ("lung" [All Fields] AND "diseases"[All Fields] AND "obstructive"[All Fields]) OR "obstructive lung diseases"[All Fields] OR ("obstructive"[All Fields] AND "pulmonary"[All Fields] AND "disease"[All Fields]) OR "obstructive pulmonary disease"[All Fields]) AND (genome-wide association study[MeSH Terms] OR (("genome-wide”[All Fields] OR ("genome”AND “wide”)) AND "association" [All Fields] AND "study"[All Fields]) OR "gwas"[All Fields] OR candidate gene[All Fields] OR candidate genes[All Fields] OR polymorphism[All Fields] OR polymorphisms[All Fields])

Web of Science: ((pulmonary disease, chronic obstructive) OR (pulmonary AND disease AND chronic AND obstructive) OR chronic obstructive pulmonary disease OR copd OR pulmonary emphysema OR emphysema OR pulmonary emphysema OR emphysema OR bronchitis, chronic OR bronchitis OR chronic bronchitis OR lung diseases, obstructive OR (lung AND diseases AND obstructive OR obstructive lung diseases OR (obstructive AND pulmonary AND disease) OR obstructive pulmonary disease)) AND ((genome-wide association study) OR ((genome-wide OR (genome AND wide) AND association AND study) OR gwas OR (candidate gene OR candidate genes) OR (polymorphism OR polymorphisms)))

Embase: ((((((pulmonary disease, chronic obstructive OR ("pulmonary" AND "disease" AND "chronic" AND "obstructive") OR "chronic obstructive pulmonary disease" OR "copd" OR pulmonary emphysema OR "emphysema" OR "pulmonary emphysema” OR "emphysema” OR bronchitis, chronic OR "bronchitis" OR "chronic bronchitis" OR lung diseases, obstructive OR ("lung" AND "diseases" AND "obstructive" OR "obstructive lung diseases" OR ("obstructive" AND "pulmonary" AND "disease”) OR "obstructive pulmonary disease”)) ))) AND ((genome-wide association study OR ((("genomewide" OR ("genome"AND "wide")) AND "association" AND "study") OR "gwas" OR candidate gene OR candidate genes) OR (polymorphism OR polymorphisms)))) \{No Related Terms\} 
Chapter 3 | Genetic, lifestyle, and environmental risk factors of COPD development

\section{Lifestyle and environmental factors}

PubMed: ("Pulmonary Disease, Chronic Obstructive"[Mesh] OR "Pulmonary Emphysema"[Mesh] OR "Bronchitis, Chronic"[Mesh]) AND ("Diet"[Mesh] OR "Exercise"[Mesh] OR "Smoking”[Mesh] OR "Environment"[Mesh] OR "Occupations"[Mesh] OR "Ethanol”[Mesh]) AND ("Meta-Analysis"[Publication Type] OR "pooled analysis")

Web of Science: ("Chronic Obstructive Pulmonary Disease" OR "COPD" OR "emphysema" OR "chronic bronchitis") AND (diet OR dietary OR nutrition* OR exercise OR "physical activity" OR smoking OR tobacco OR environment* OR occupation* OR alcohol OR alcoholic) AND ("Meta-Analysis" OR "pooled analysis")

Embase: (chronic obstructive lung disease/ OR emphysema/ OR chronic bronchitis/) AND (diet/ OR dietary intake/ OR nutrition/ OR exercise/ OR physical activity/ OR smoking/ OR tobacco/ OR tobacco consumption/ OR environment/ OR occupation/ OR alcohol/ OR alcohol consumption/) AND (meta analysis/ OR "pooled analysis") 


\section{Supplementary figure 1}

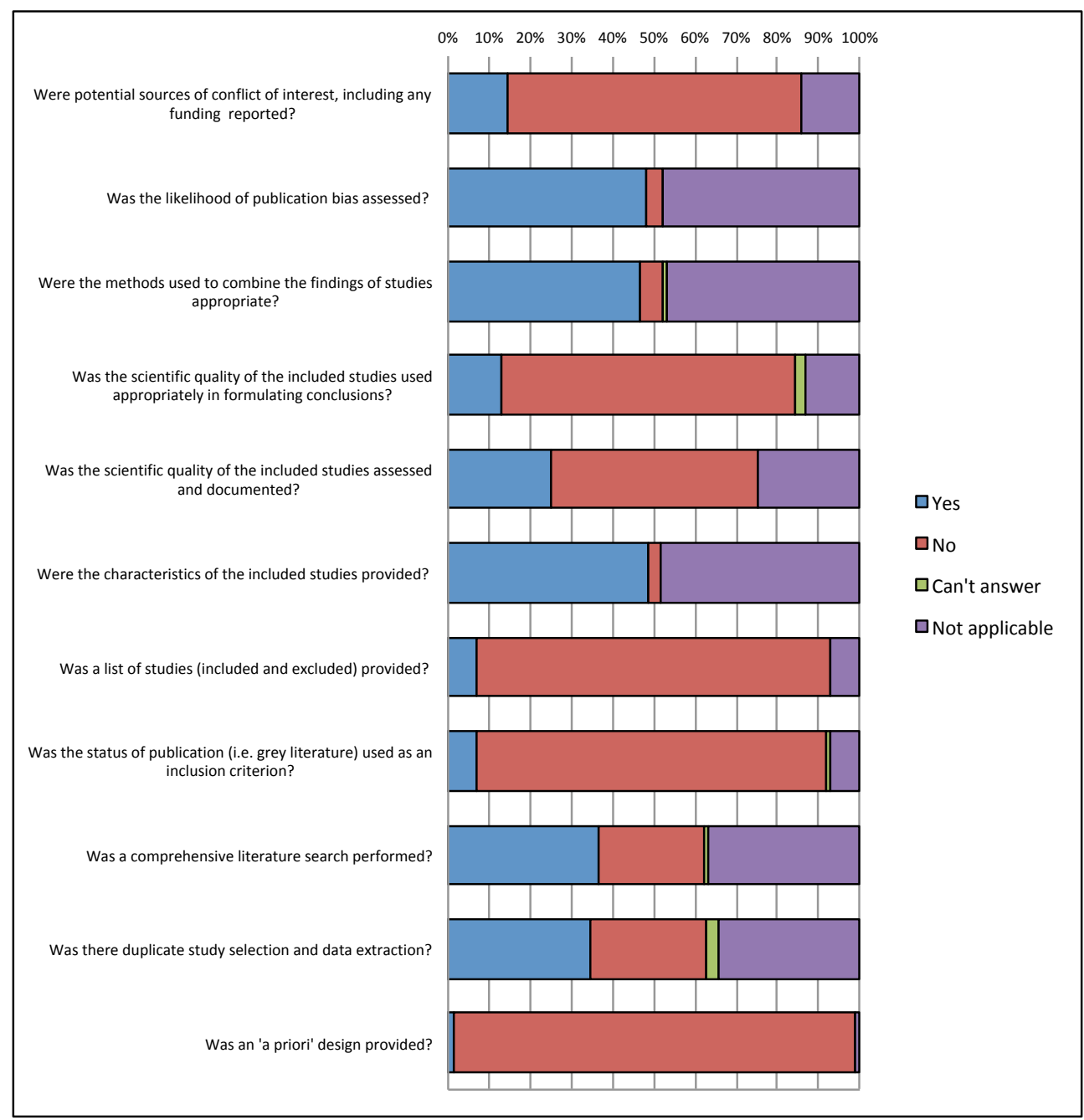

Supplementary figure 1 Summary of AMSTAR ratings per item for genetic and lifestyle/ environmental factors combined 
Chapter 3 | Genetic, lifestyle, and environmental risk factors of COPD development

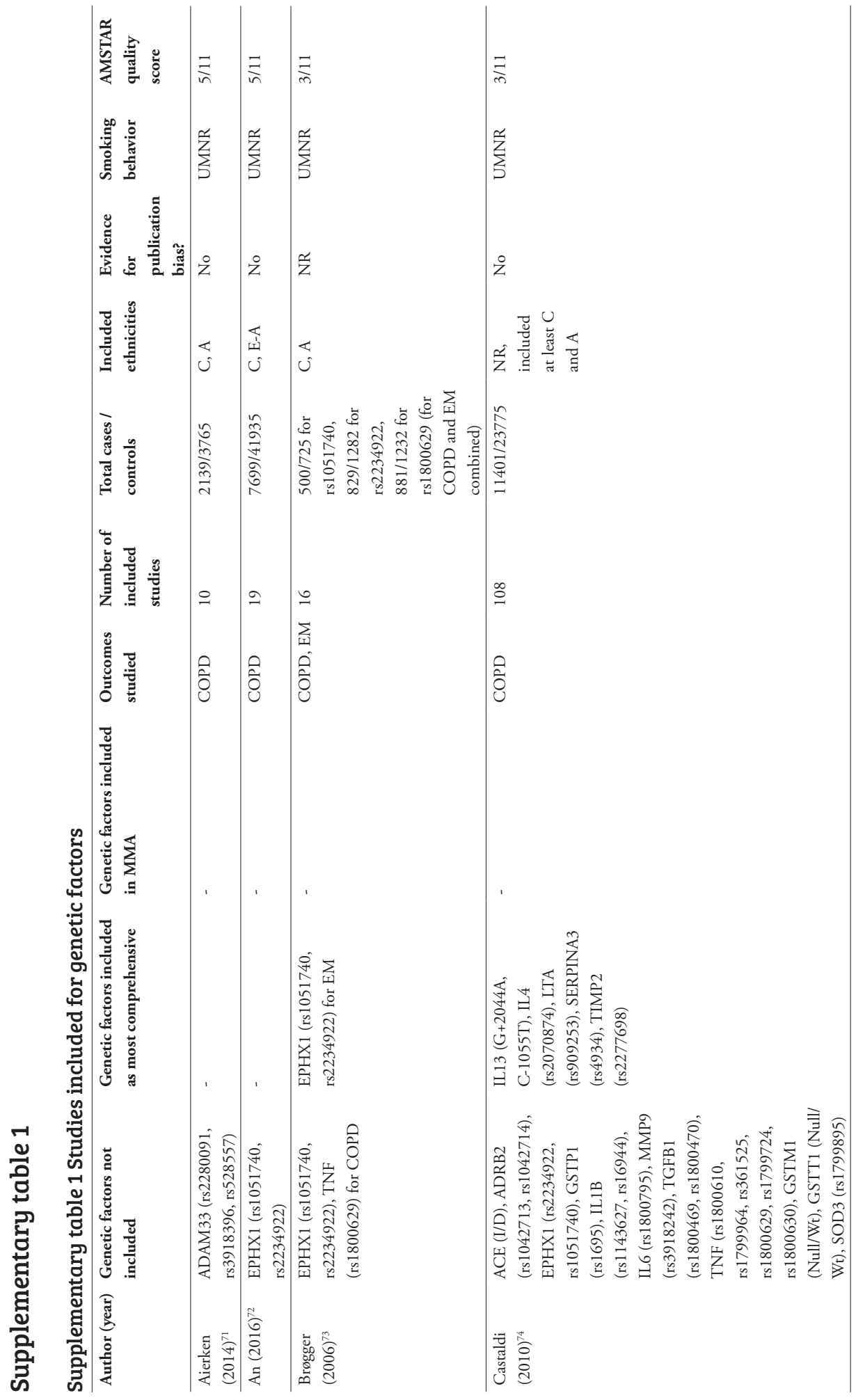


Chapter 3 | Genetic, lifestyle, and environmental risk factors of COPD development

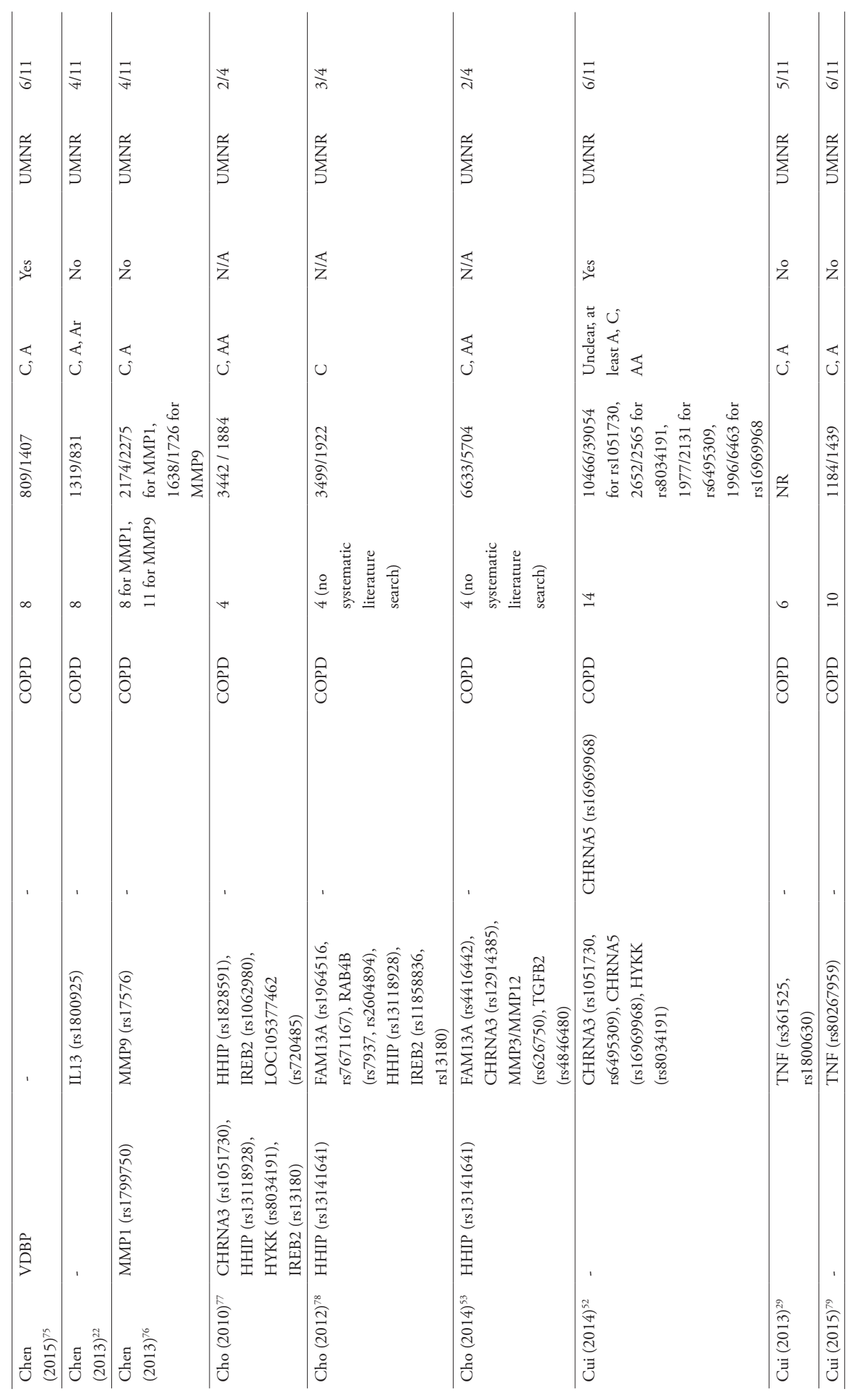


Chapter 3 | Genetic, lifestyle, and environmental risk factors of COPD development

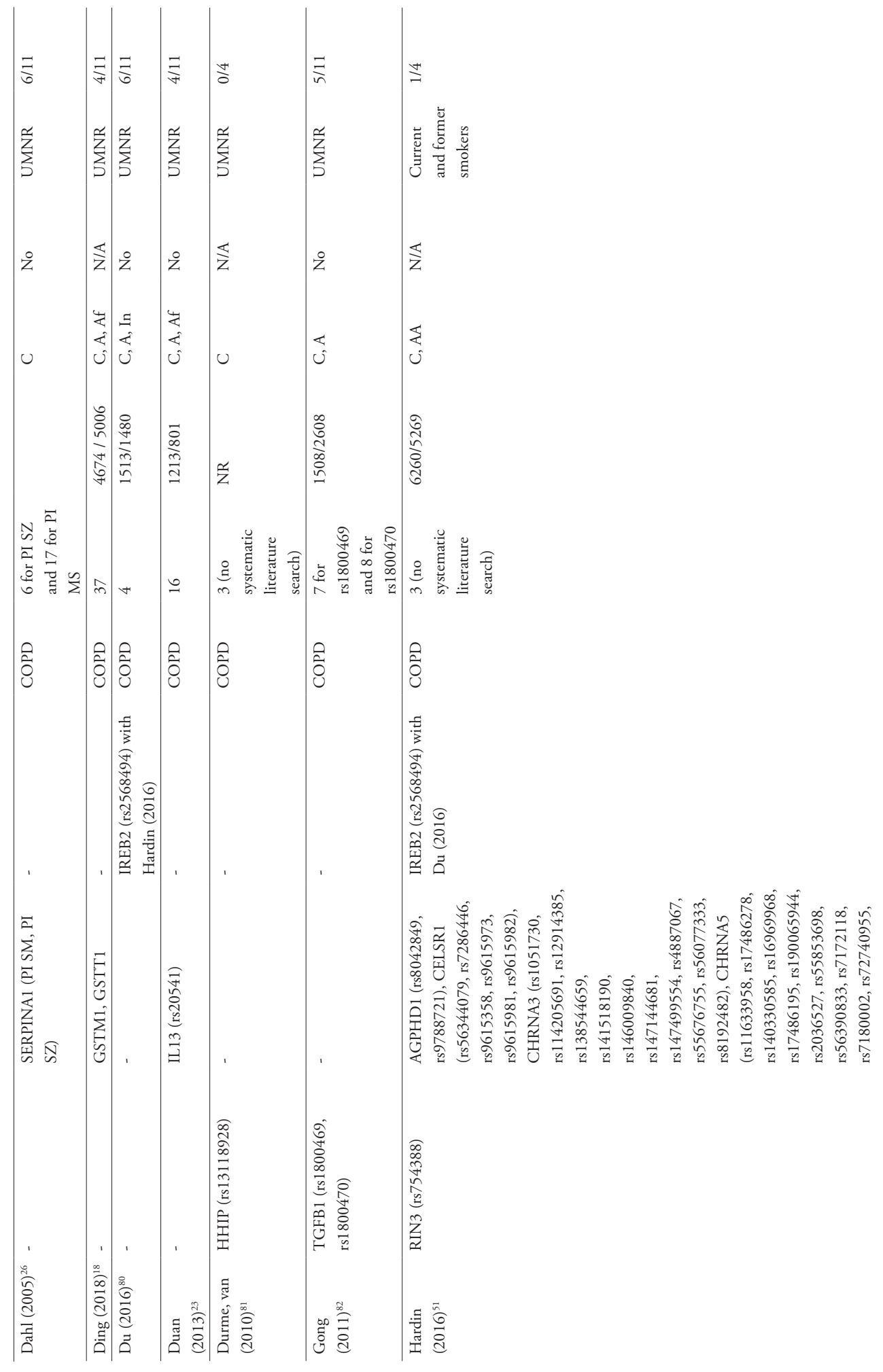


Chapter 3 | Genetic, lifestyle, and environmental risk factors of COPD development

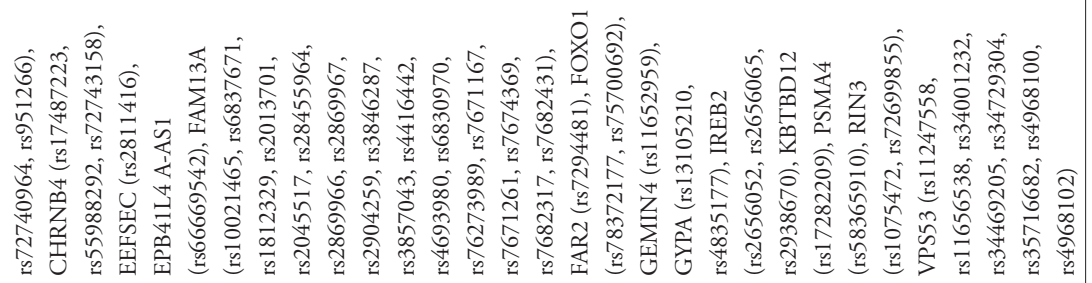


Chapter 3 | Genetic, lifestyle, and environmental risk factors of COPD development

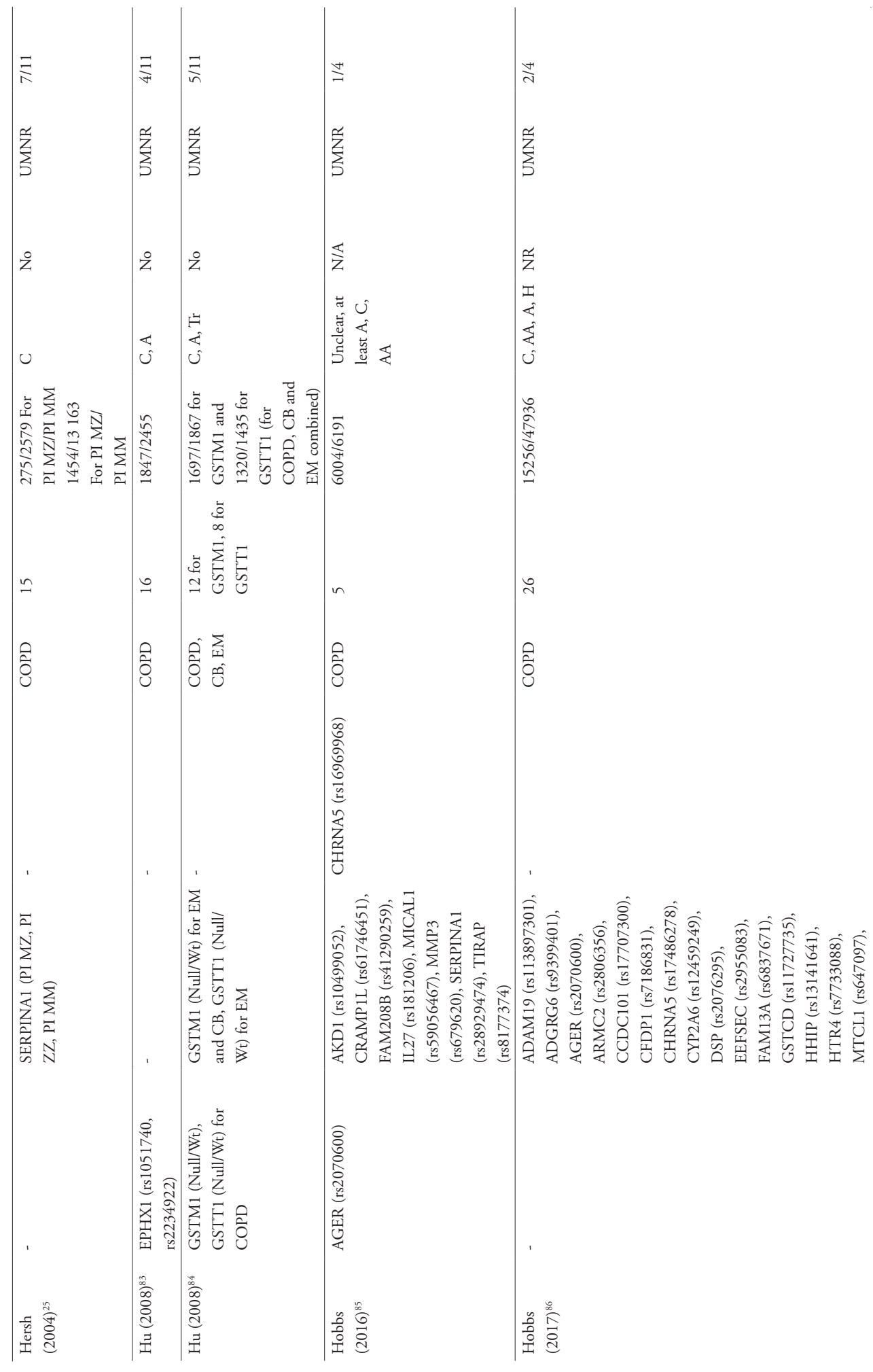


Chapter 3 | Genetic, lifestyle, and environmental risk factors of COPD development

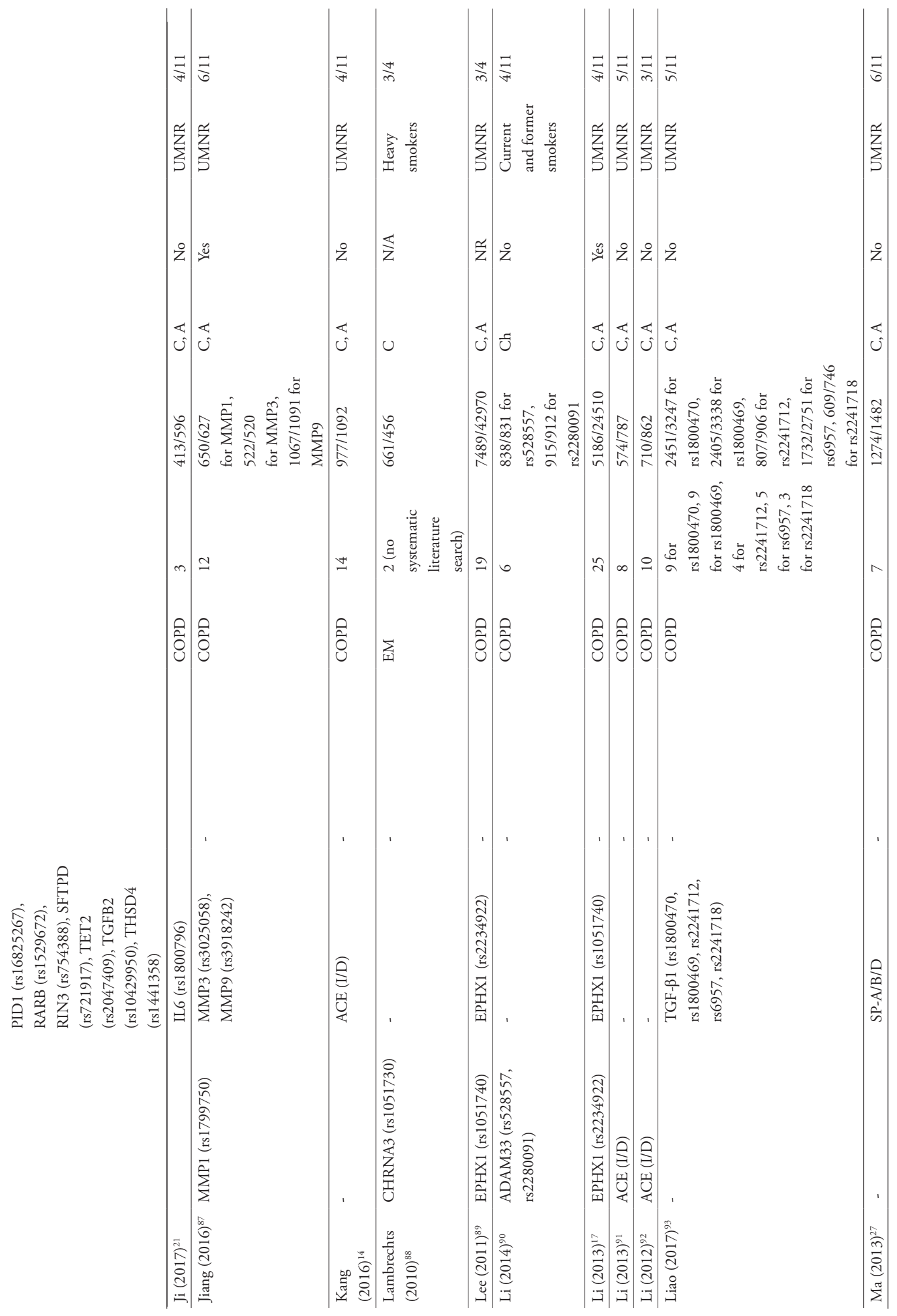


Chapter 3 | Genetic, lifestyle, and environmental risk factors of COPD development

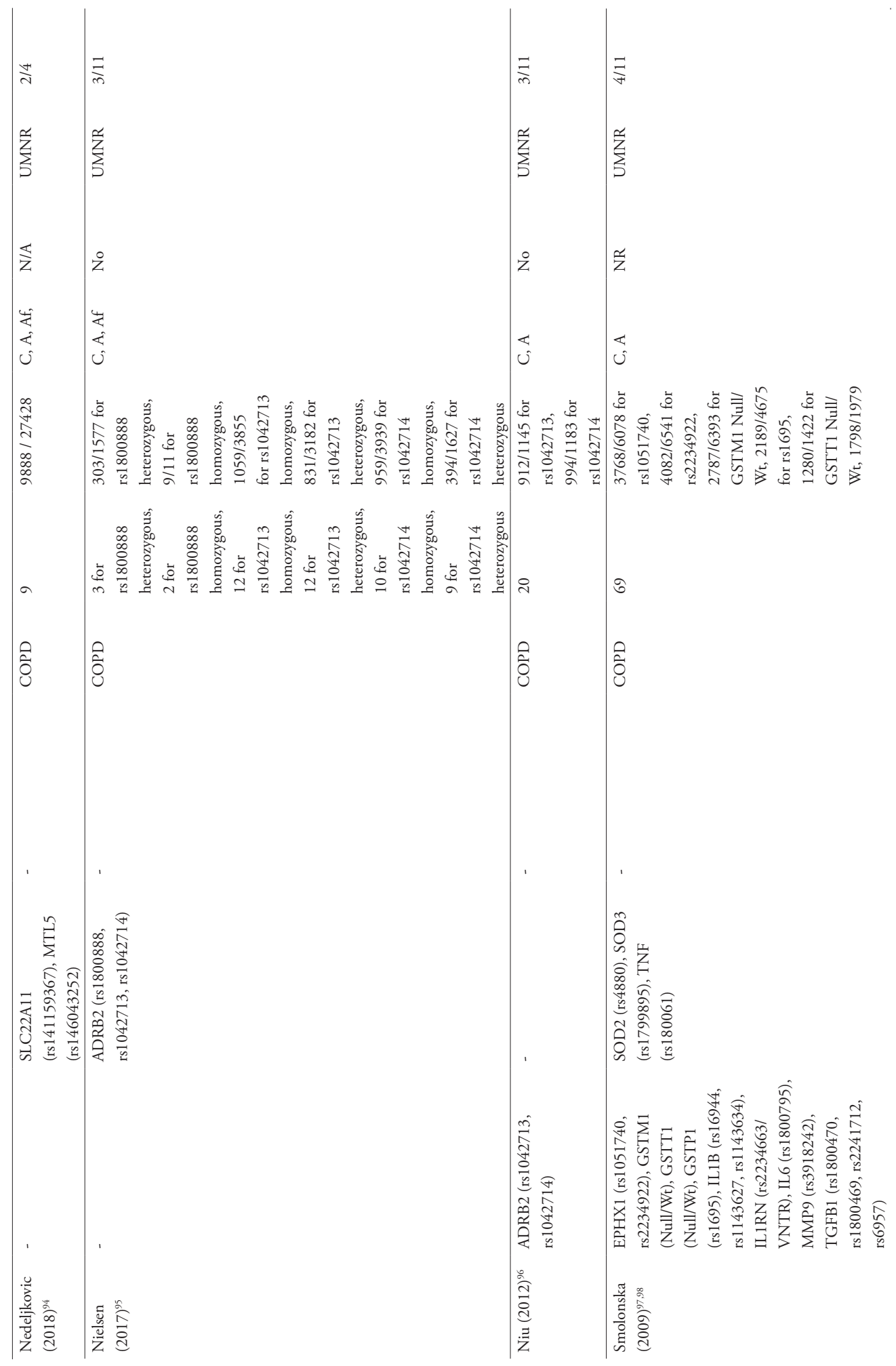


Chapter 3 | Genetic, lifestyle, and environmental risk factors of COPD development

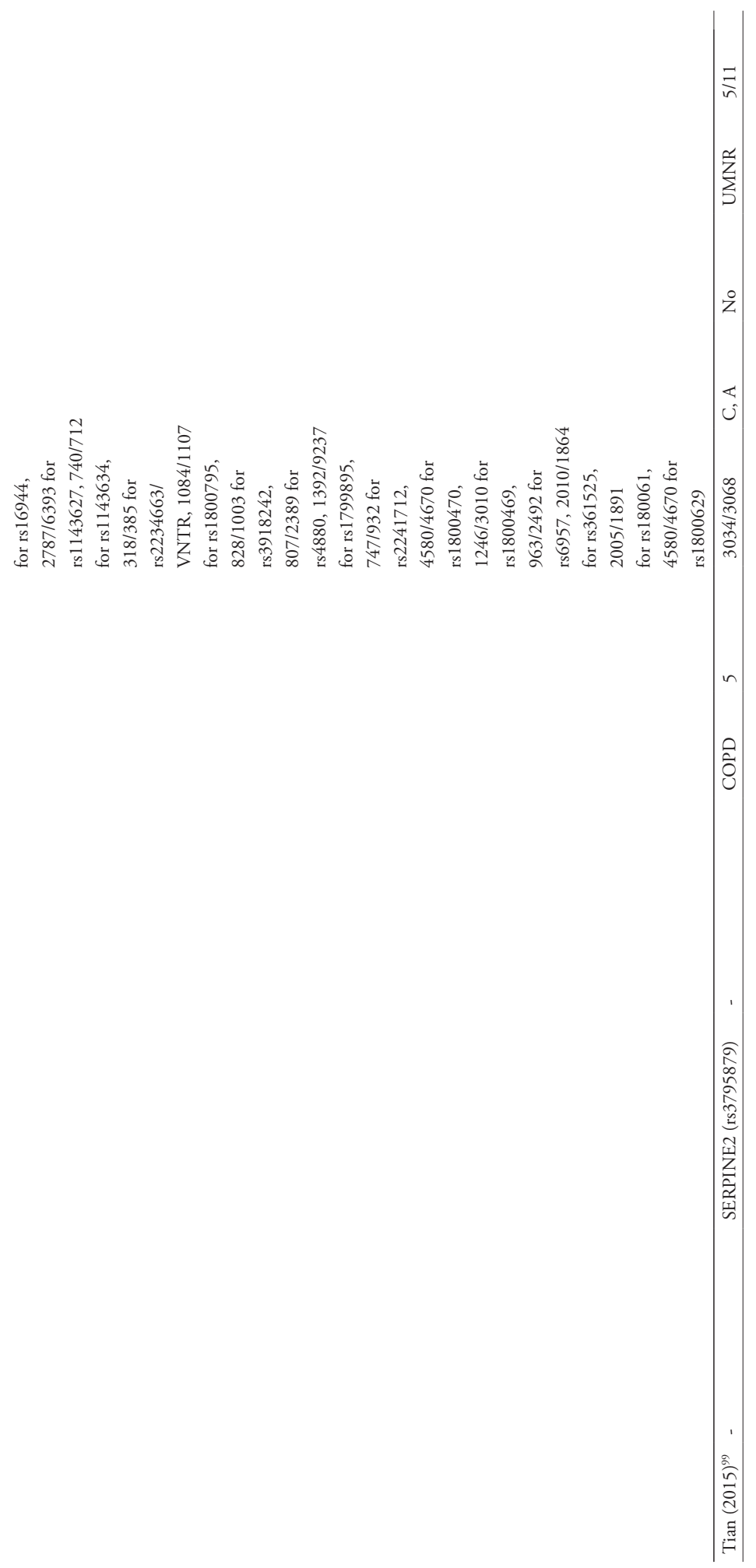


Chapter 3 | Genetic, lifestyle, and environmental risk factors of COPD development

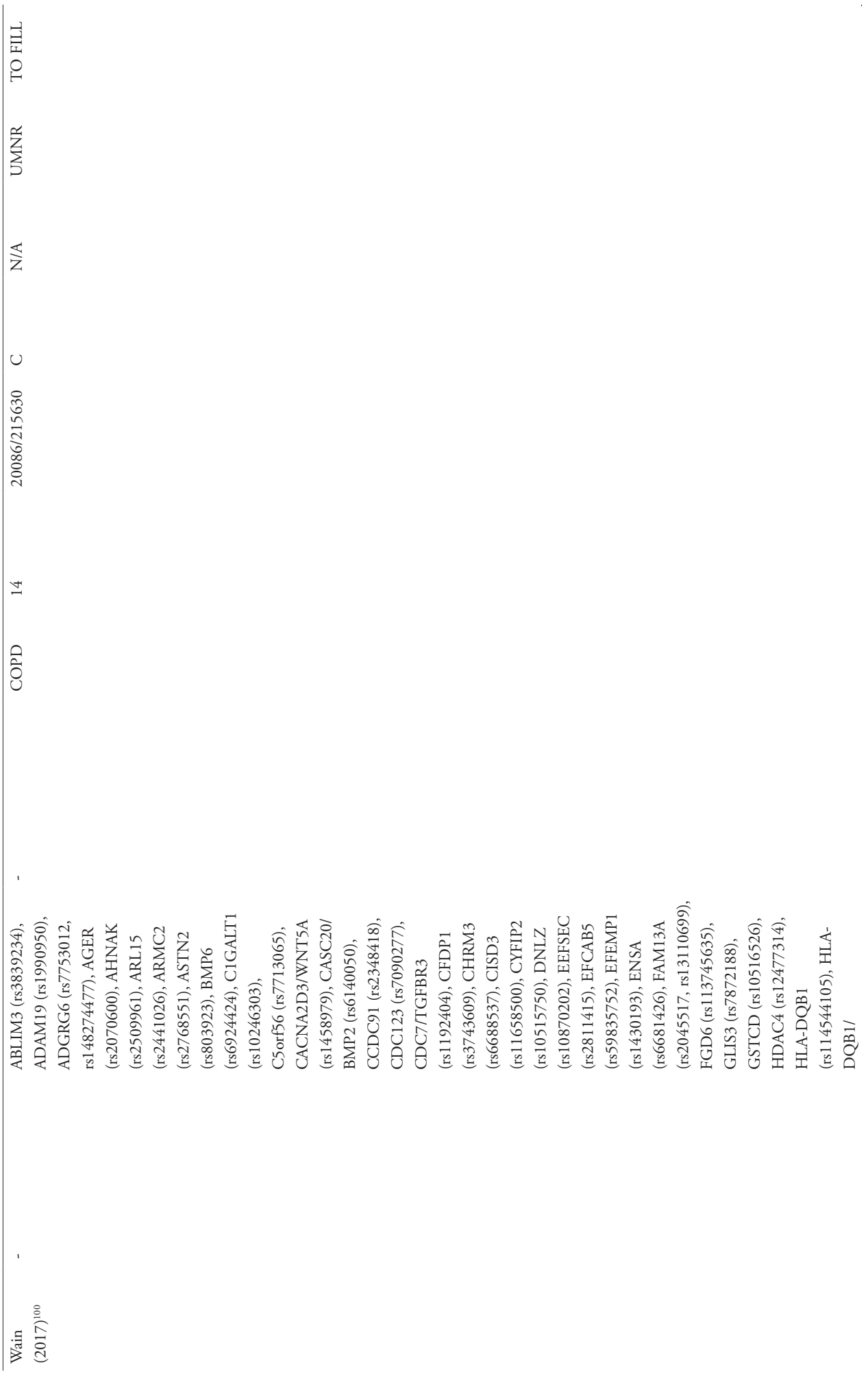


Chapter 3 | Genetic, lifestyle, and environmental risk factors of COPD development

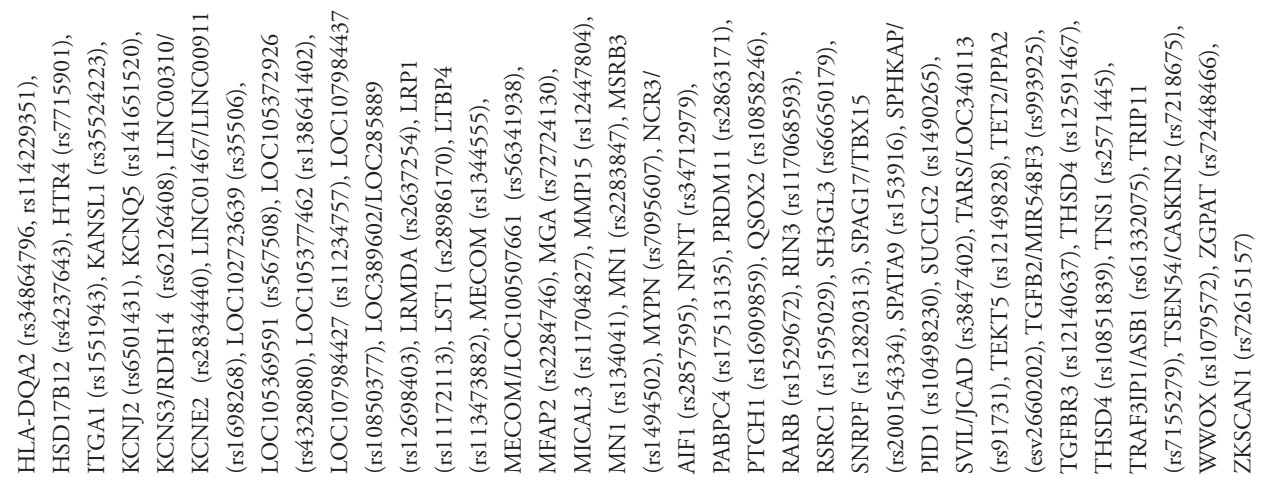


Chapter 3 | Genetic, lifestyle, and environmental risk factors of COPD development

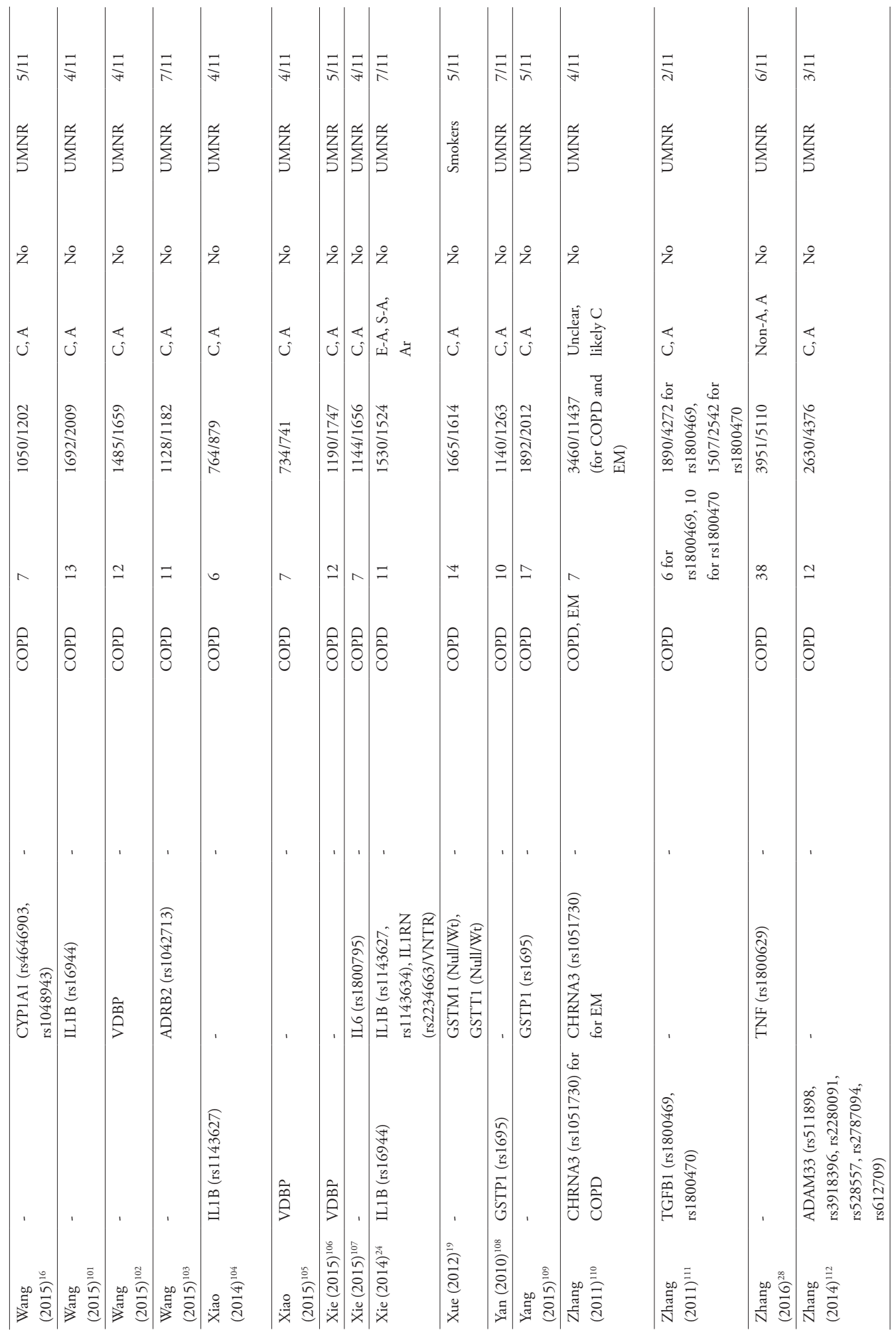


Chapter 3 | Genetic, lifestyle, and environmental risk factors of COPD development

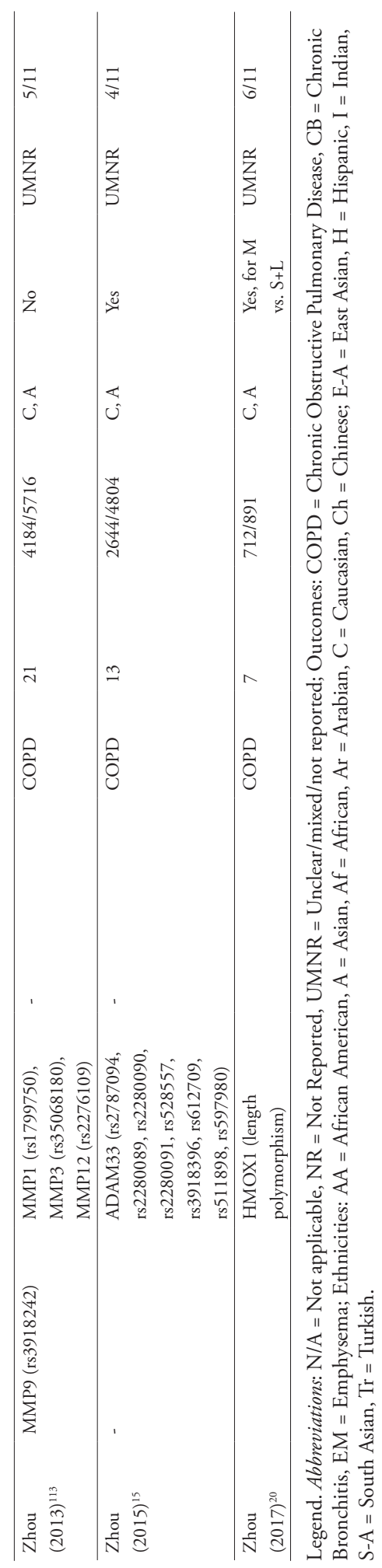




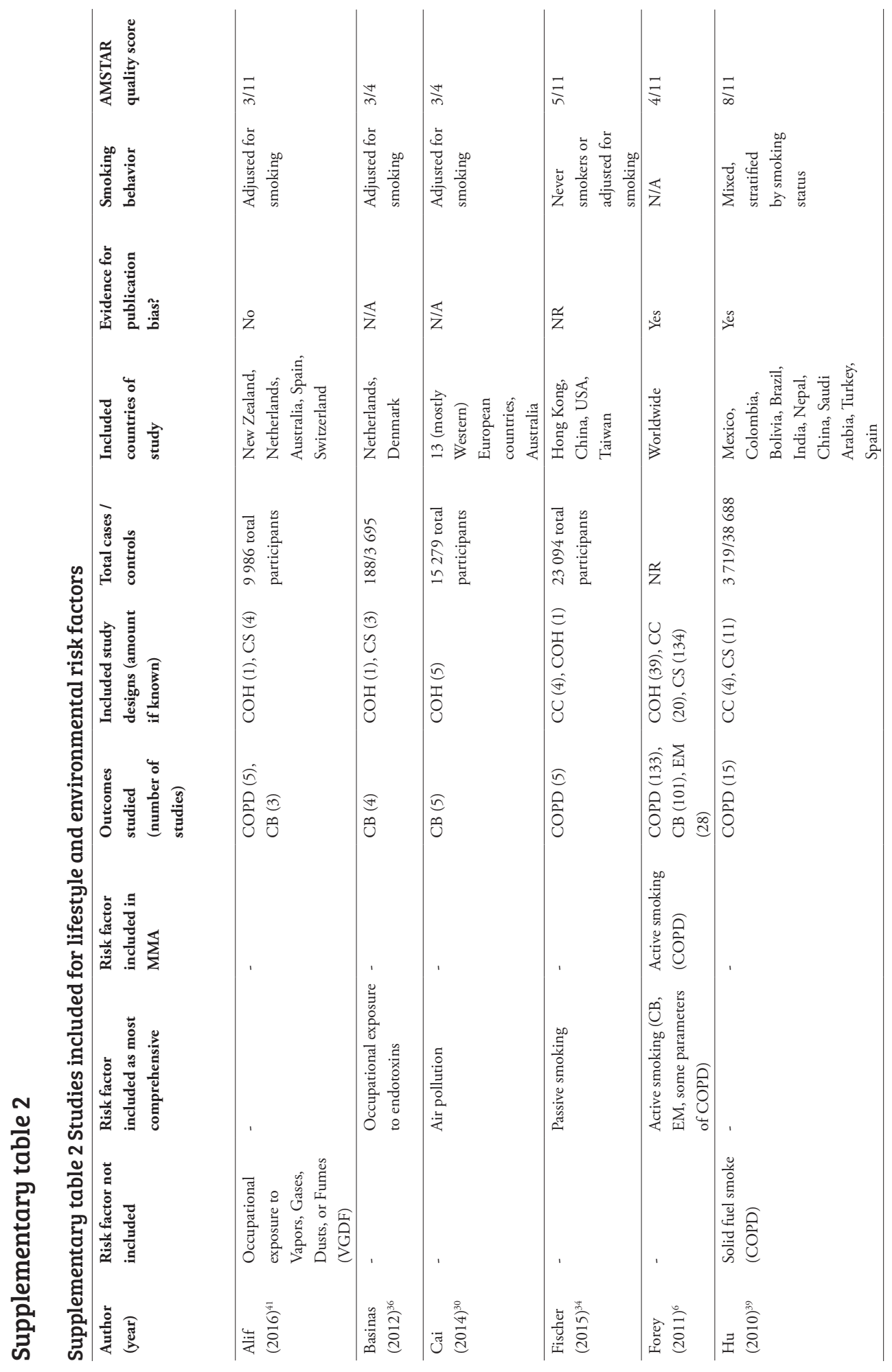


Chapter 3 | Genetic, lifestyle, and environmental risk factors of COPD development

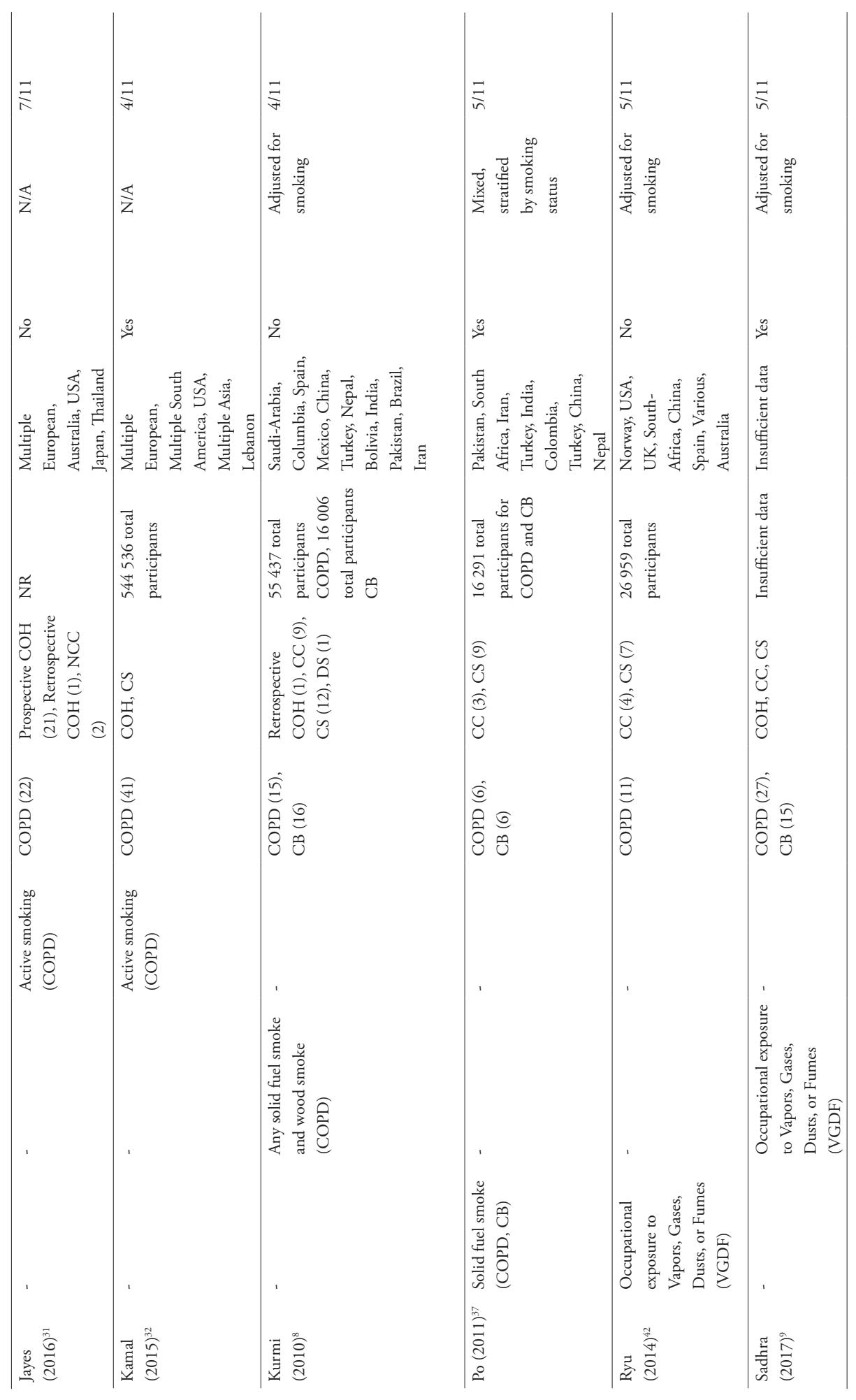


Chapter 3 | Genetic, lifestyle, and environmental risk factors of COPD development

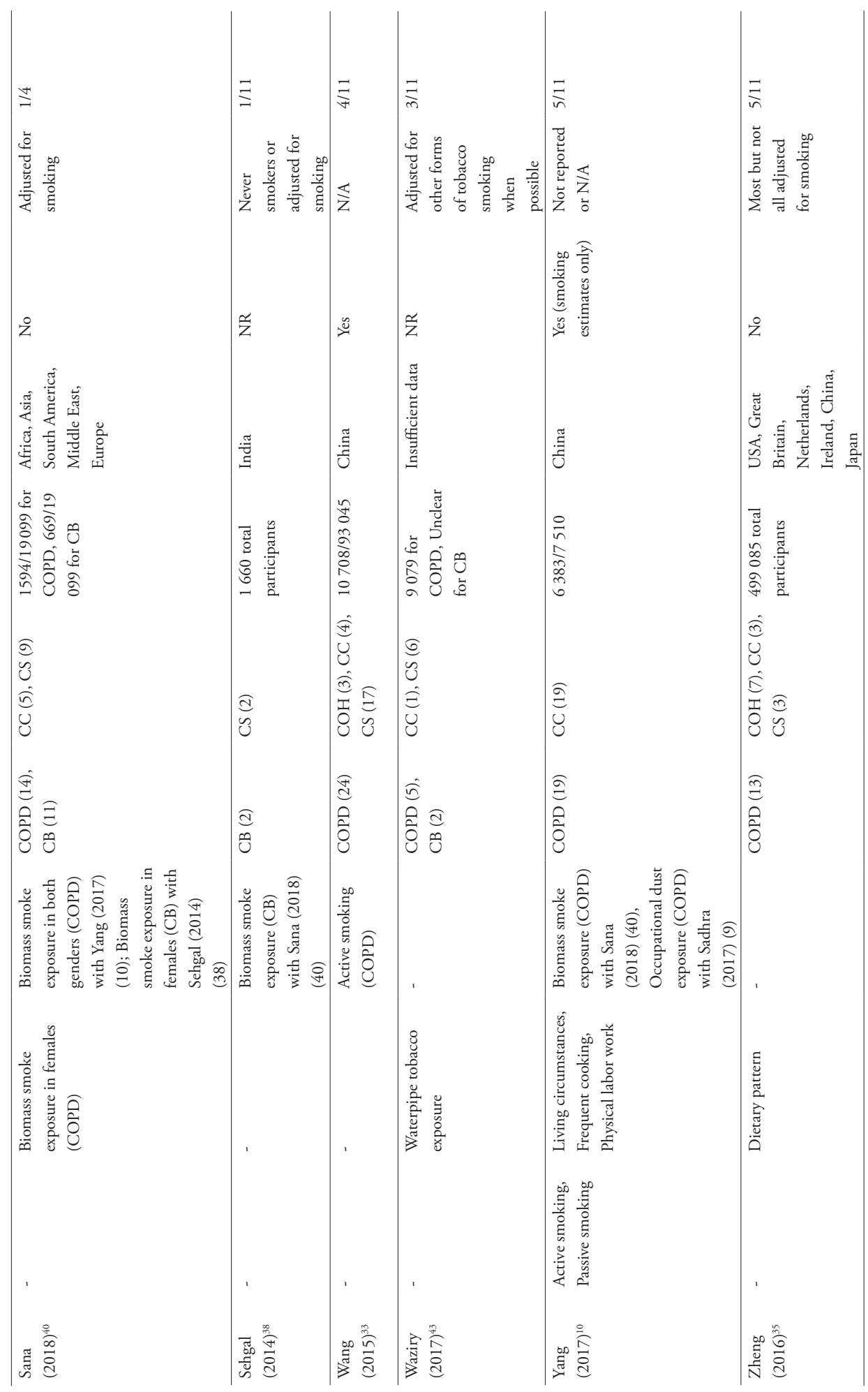


Chapter 3 | Genetic, lifestyle, and environmental risk factors of COPD development

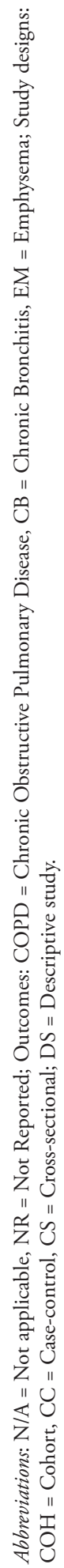


Chapter 3 | Genetic, lifestyle, and environmental risk factors of COPD development

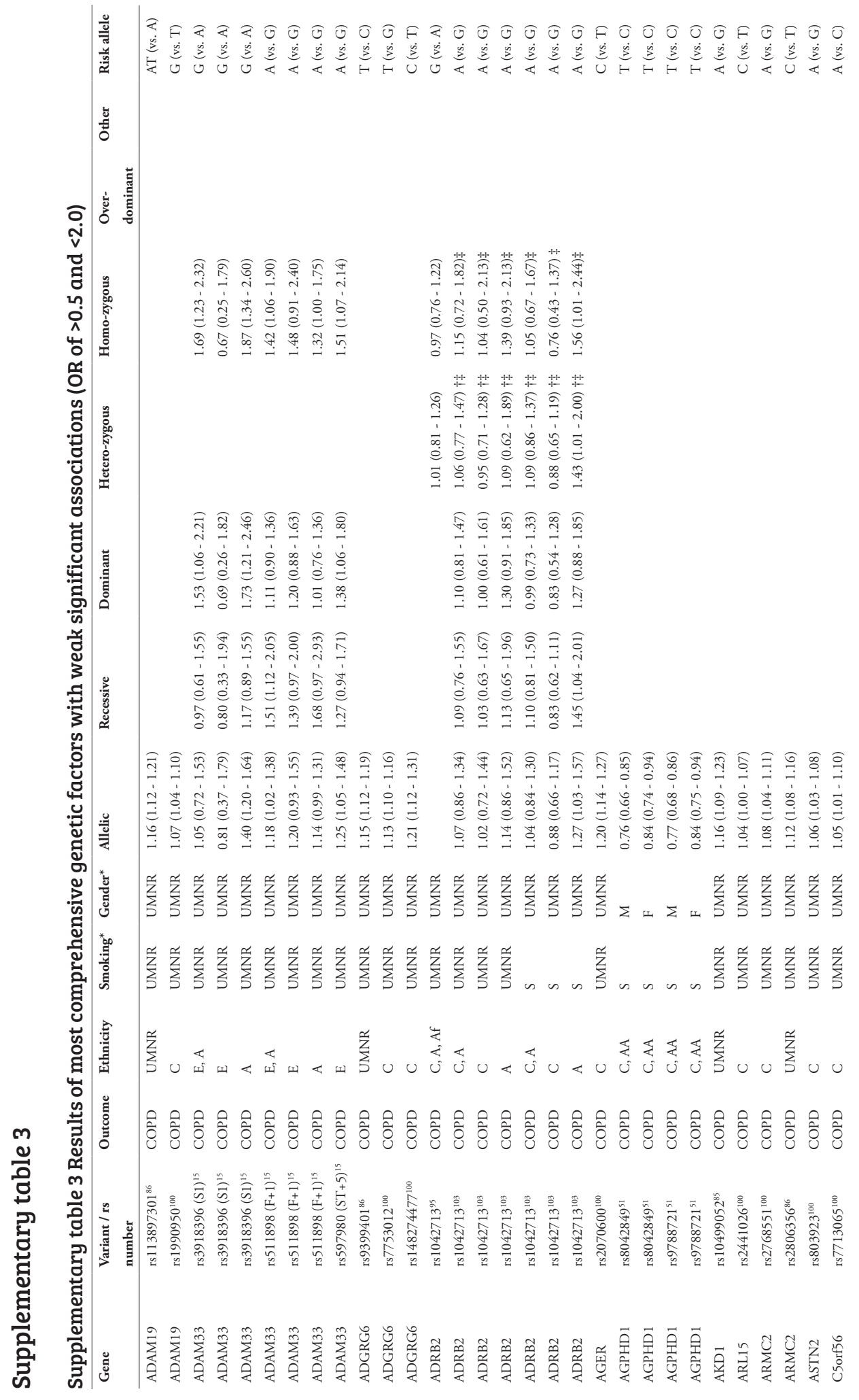


Chapter 3 | Genetic, lifestyle, and environmental risk factors of COPD development

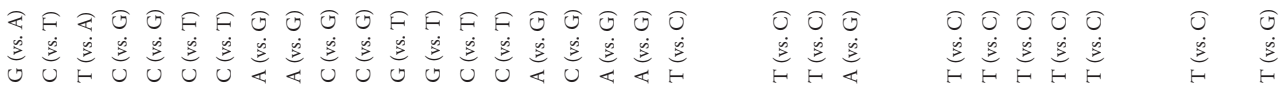

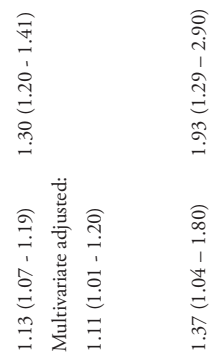

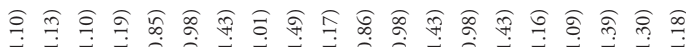

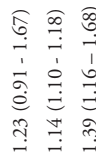

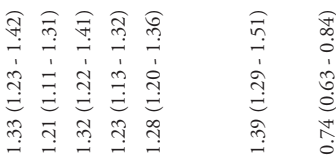

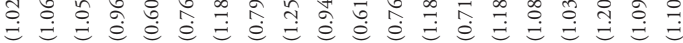

㽞

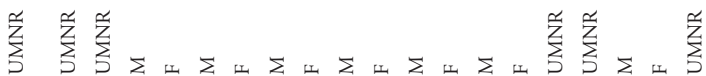

$\sum_{S}^{n} \sum_{S}^{n} \sum_{S}^{n}$

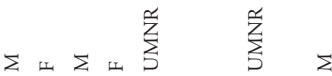

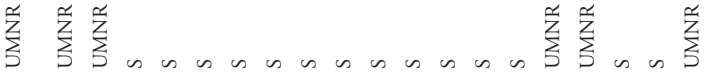

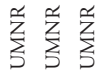

is n s is $\sum_{5}^{\frac{a}{\zeta}}$ is

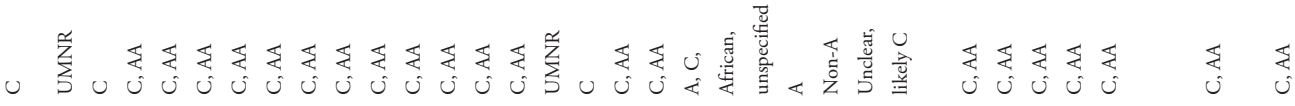

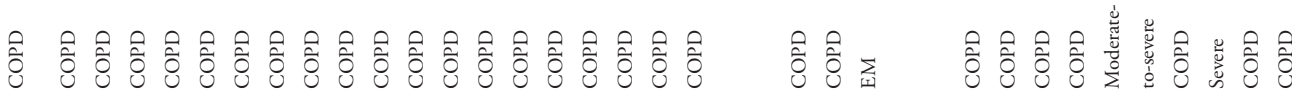

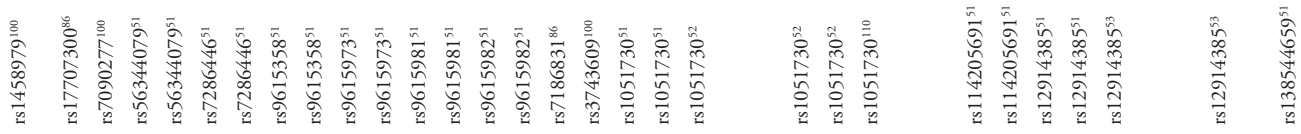

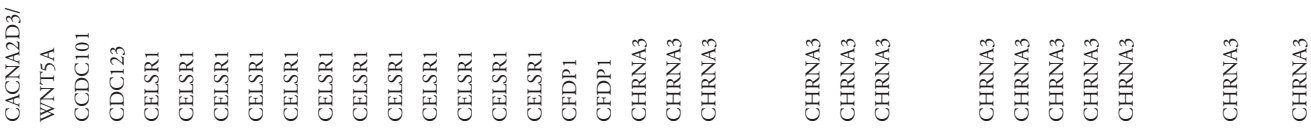


Chapter 3 | Genetic, lifestyle, and environmental risk factors of COPD development

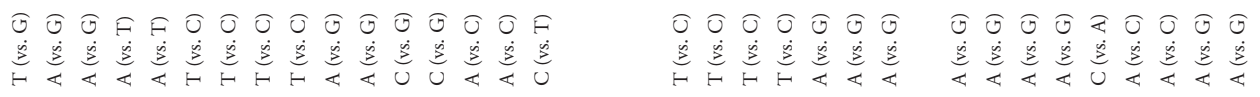

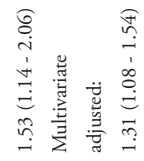

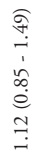

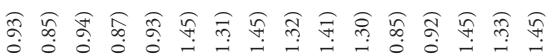

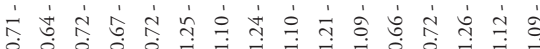

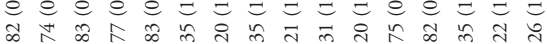

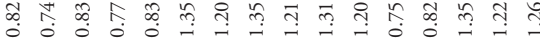
ш

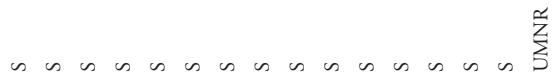

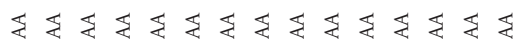

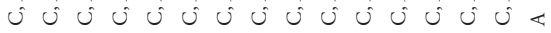

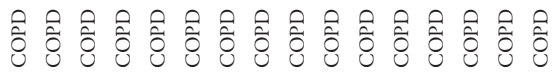

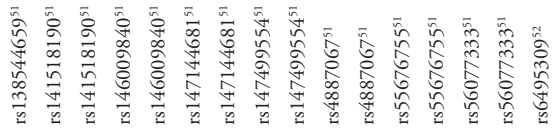

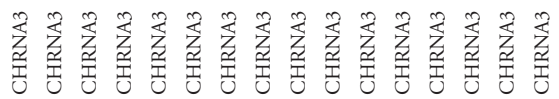

ঔ

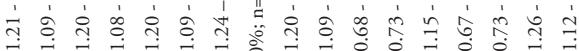
ज.

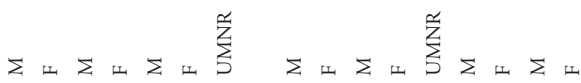

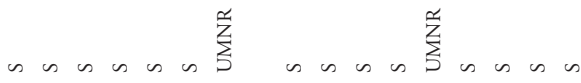

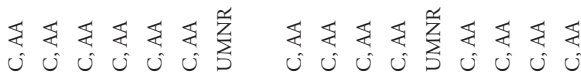

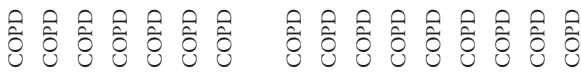

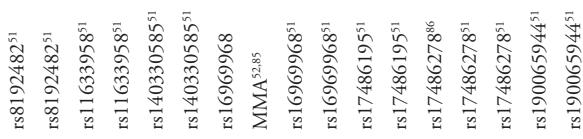

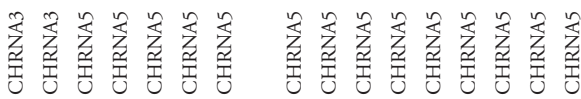




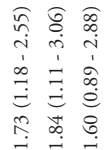

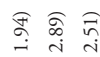

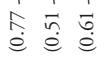

$$
\begin{aligned}
& \text { กิ กิ ปู } \\
& \text { ิㅡㅇㅝ }
\end{aligned}
$$

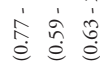

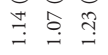

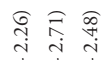

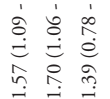

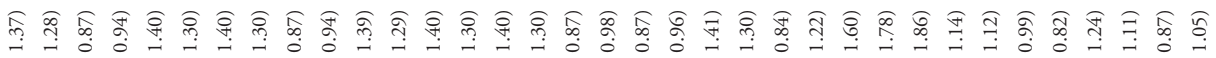

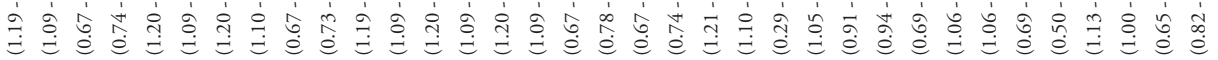
ঙำ

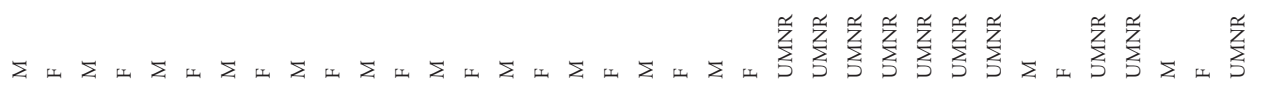

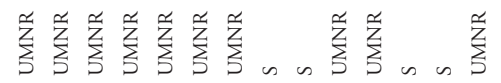

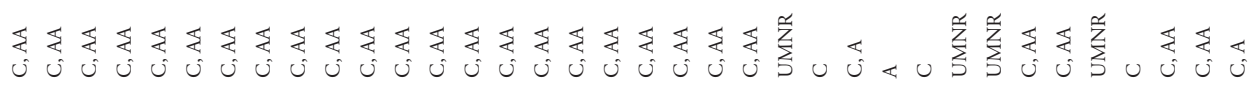

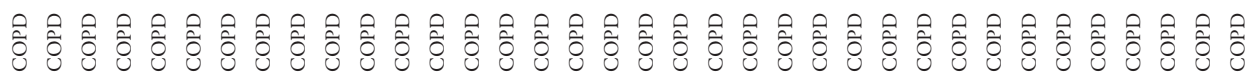

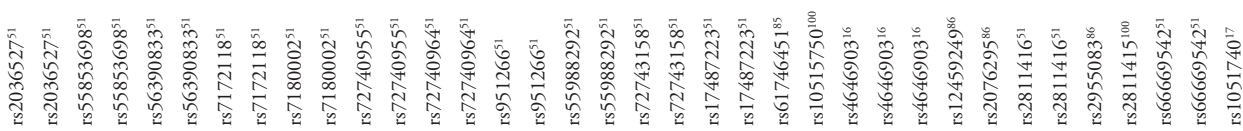

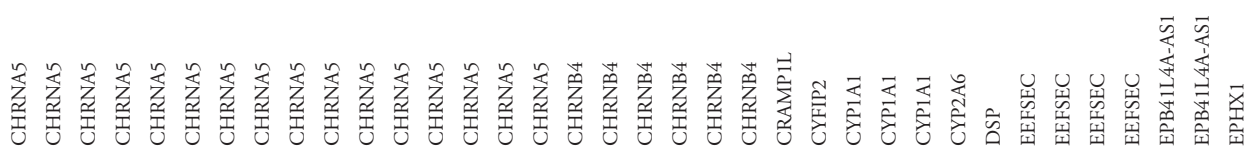


Chapter 3 | Genetic, lifestyle, and environmental risk factors of COPD development

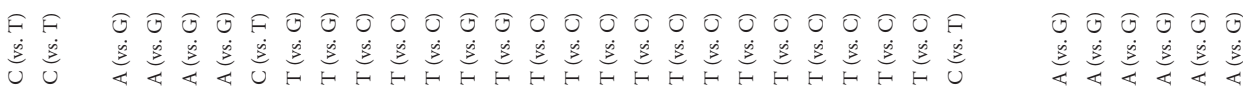

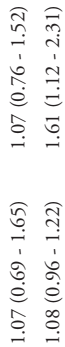

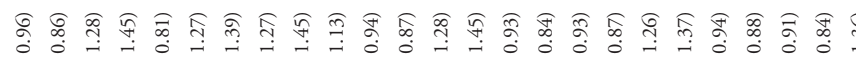

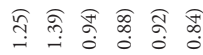



\& ते

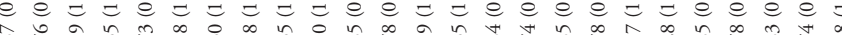

$\Xi \doteq \dot{e} \doteq$

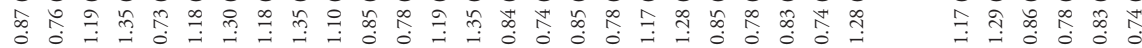

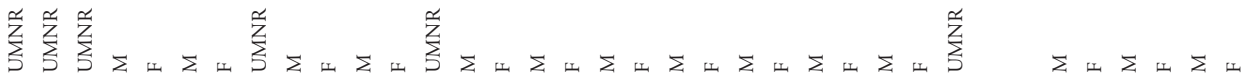

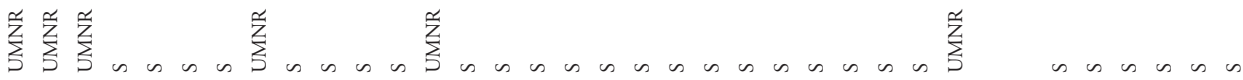

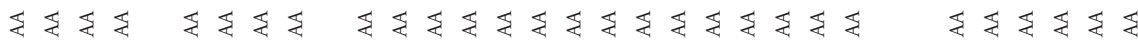

« «

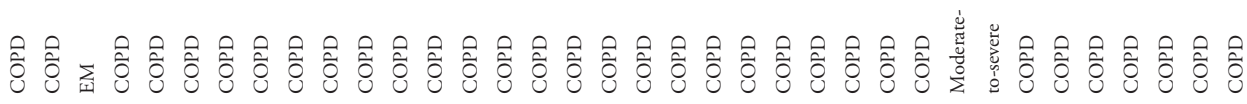

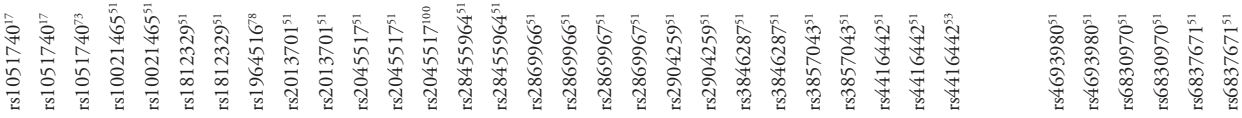

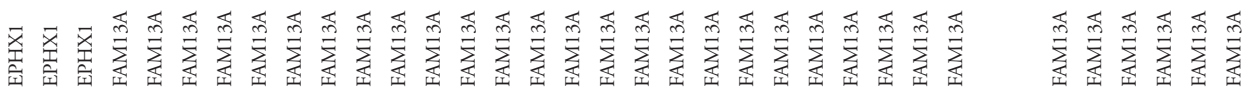


Chapter 3 | Genetic, lifestyle, and environmental risk factors of COPD development

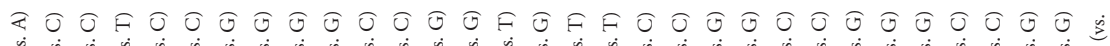

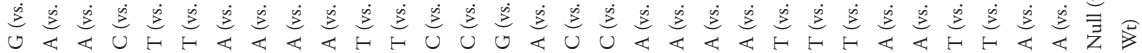

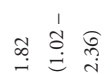

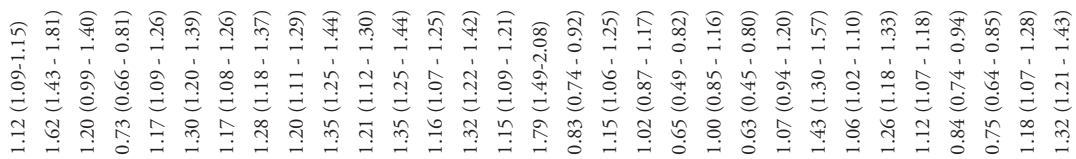

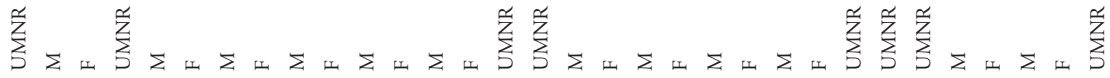

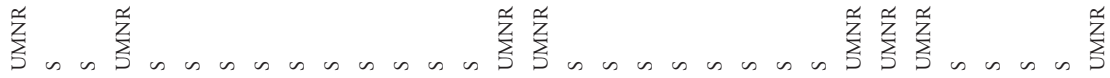

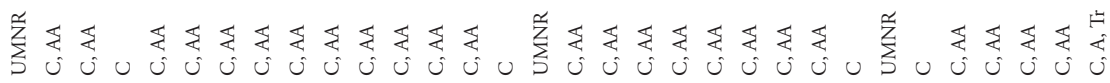

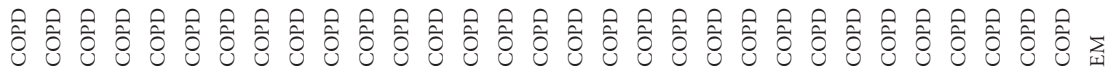

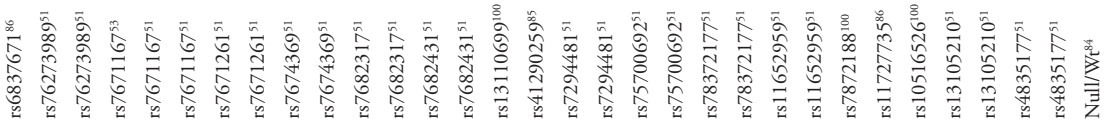

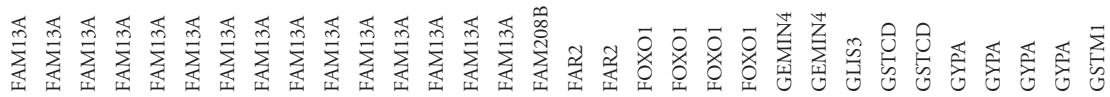


Chapter 3 | Genetic, lifestyle, and environmental risk factors of COPD development

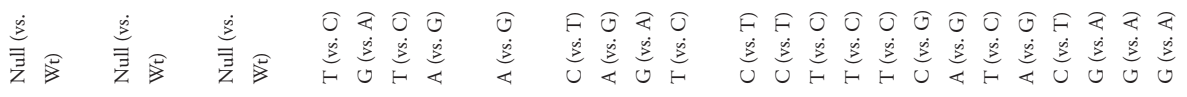

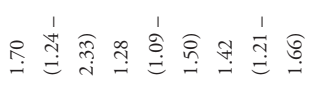

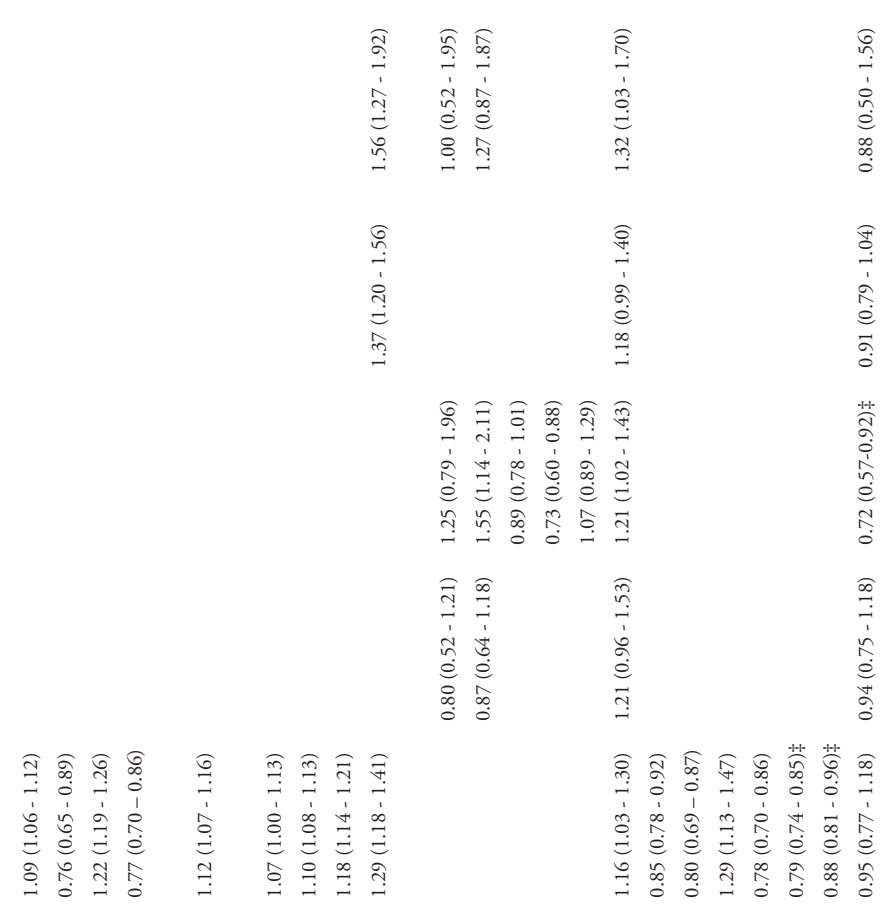

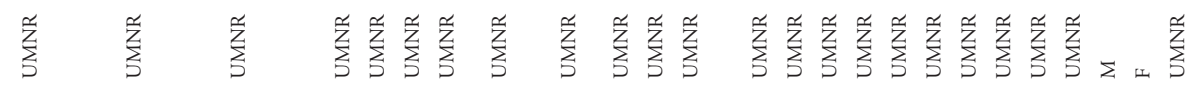

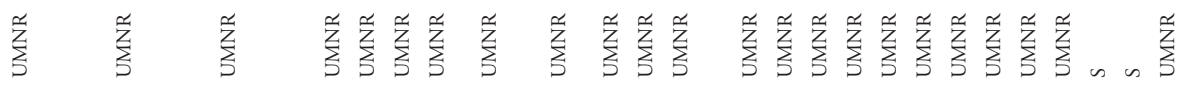

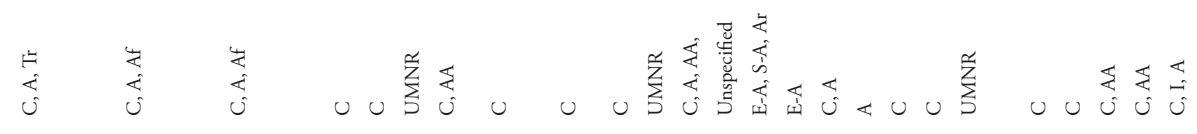

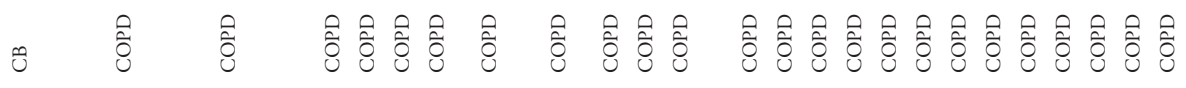

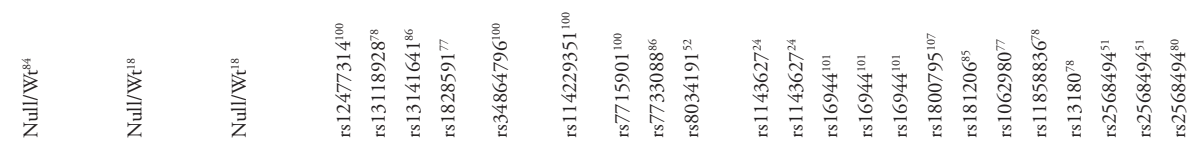

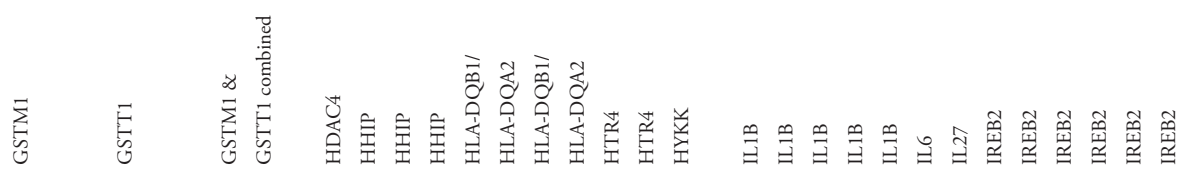


Chapter 3 | Genetic, lifestyle, and environmental risk factors of COPD development

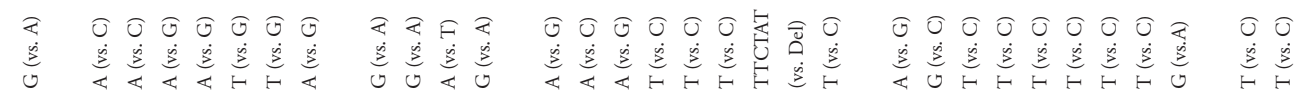

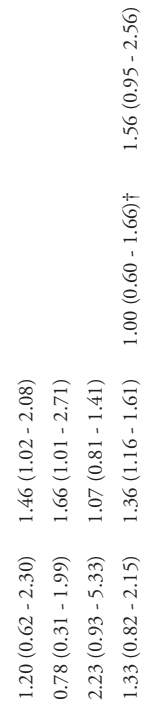

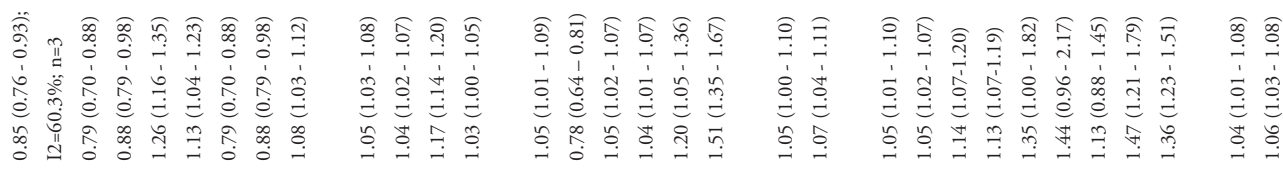

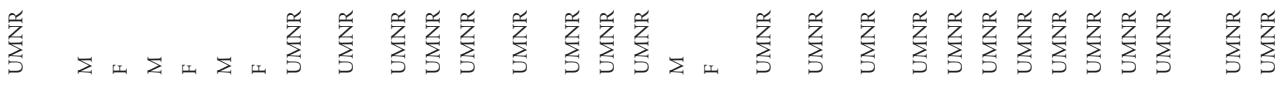

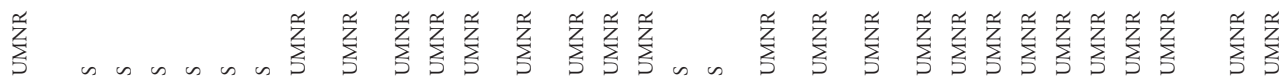

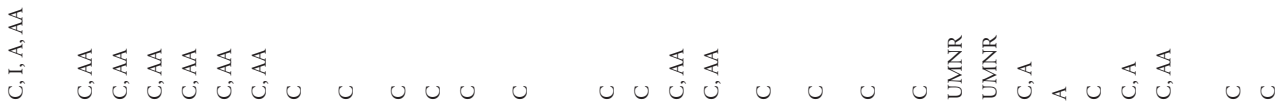

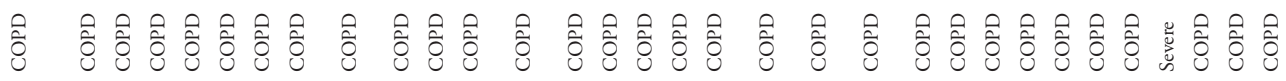

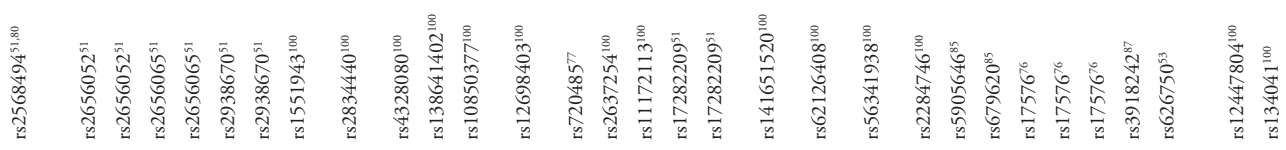

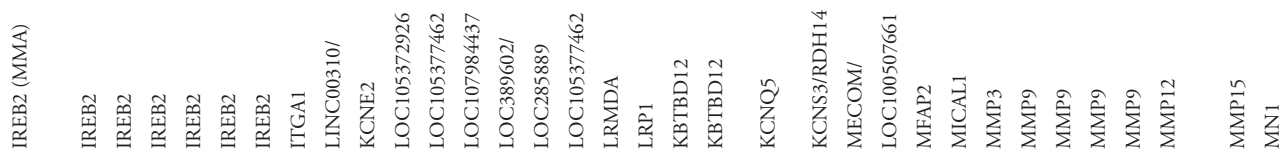


Chapter 3 | Genetic, lifestyle, and environmental risk factors of COPD development

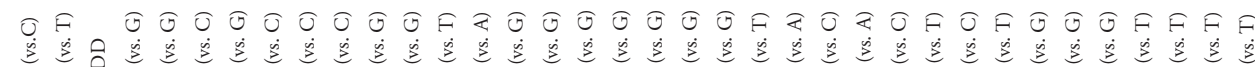

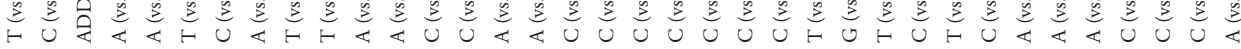
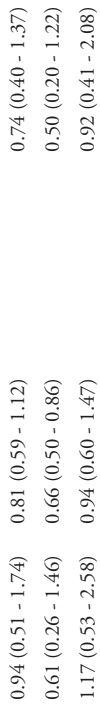

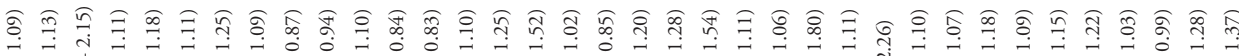

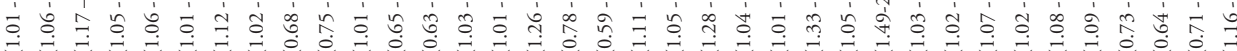

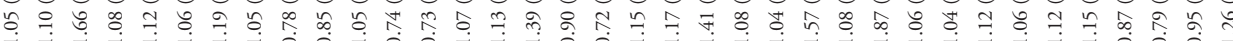

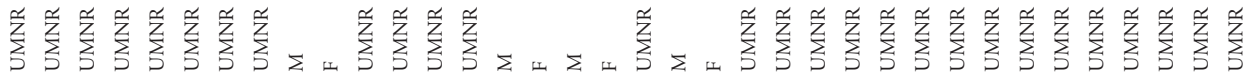

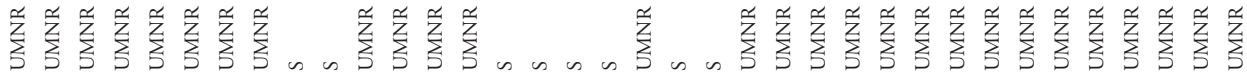

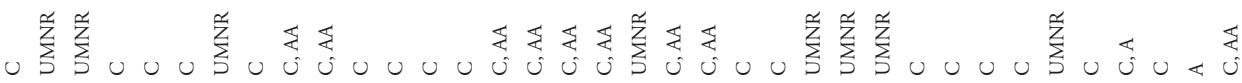

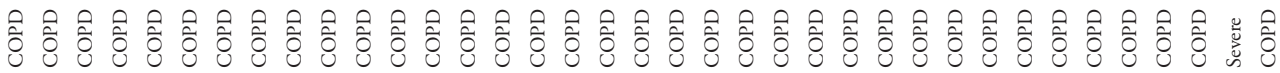

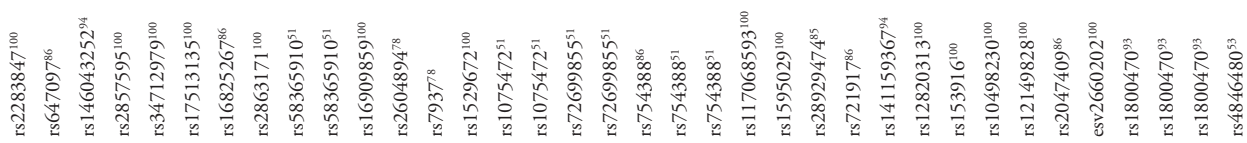

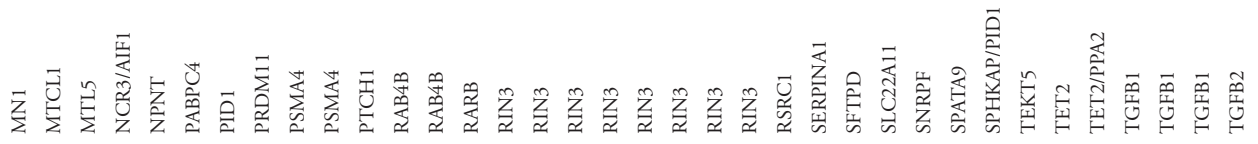


Chapter 3 | Genetic, lifestyle, and environmental risk factors of COPD development

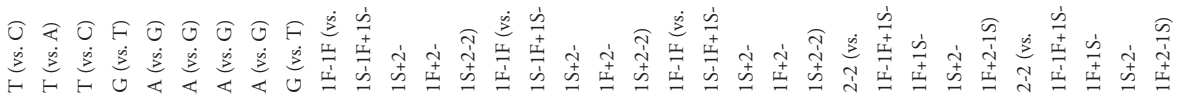

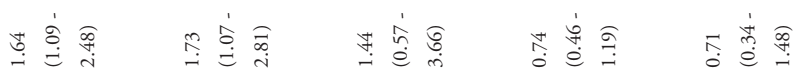

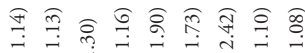

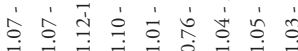

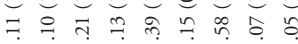

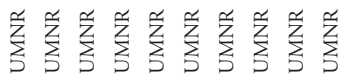

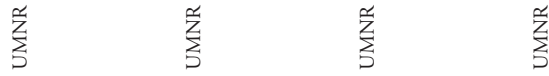

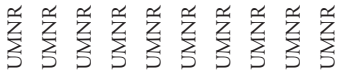

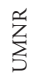

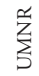

$\sum_{j}^{\sim} \quad \sum_{5}^{\infty}$

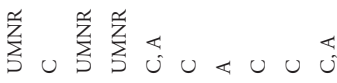

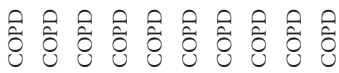

วิ

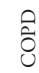

玄

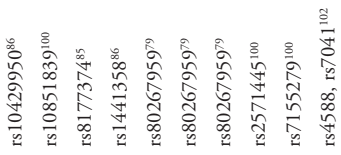

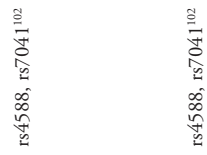

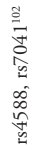

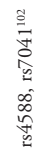

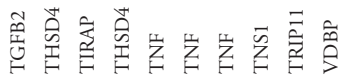

$\stackrel{\tilde{m}}{\stackrel{\rho}{s}}$

$\stackrel{\hat{\omega}}{\rightrightarrows}$

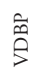

商 
Chapter 3 | Genetic, lifestyle, and environmental risk factors of COPD development

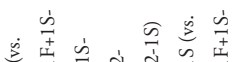

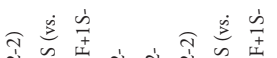

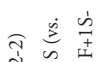

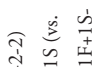

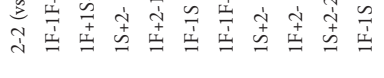

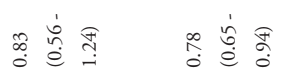

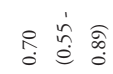

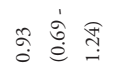

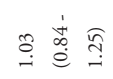
o

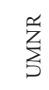

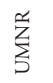

$\sum_{5}^{a}$

$\sum_{5}^{n}$

$\sum_{5}^{a}$

$\sum_{5}^{\infty}$

莣

艺

$\sum_{5}^{a}$

荒

苂

0
0
0
0
0
0
0
0
0
0
0
0
0
0
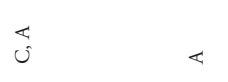

ิิ

ิิ

ิิ

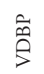

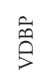

$\stackrel{\hat{ิ}}{s}$

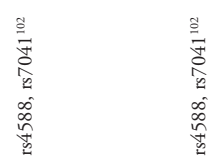


Chapter 3 | Genetic, lifestyle, and environmental risk factors of COPD development

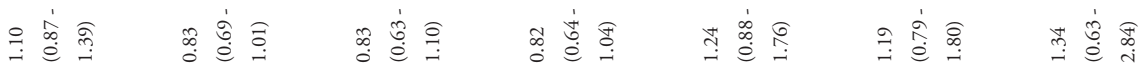

\begin{tabular}{|c|c|c|c|c|c|}
\hline 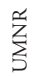 & $\sum_{\vdots}^{a}$ & $\sum_{S}^{\mathfrak{z}}$ & $\sum_{\grave{\zeta}}$ & 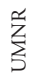 & 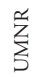 \\
\hline$\sum_{S}^{a}$ & $\sum_{S}^{\infty}$ & $\sum_{S}^{a}$ & 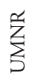 & $\sum_{S}^{n}$ & $\sum_{S}^{a}$ \\
\hline$u$ & $\begin{array}{l}\varangle \\
u\end{array}$ & $\varangle$ & $u$ & $\begin{array}{l}\varangle \\
ن\end{array}$ & $\varangle$ \\
\hline ڤิ & ڤิ & Оิ & $\stackrel{0}{0}$ & )ิ & $\stackrel{0}{\hat{0}}$ \\
\hline 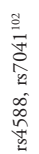 & 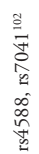 & 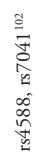 & 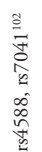 & 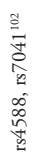 & 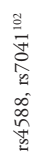 \\
\hline
\end{tabular}

\begin{tabular}{|c|c|}
\hline$\stackrel{\tilde{\hat{m}}}{\hat{\rho}}$ & $\stackrel{\overrightarrow{\tilde{\rho}}}{\rho}$ \\
\hline
\end{tabular}


Chapter 3 | Genetic, lifestyle, and environmental risk factors of COPD development

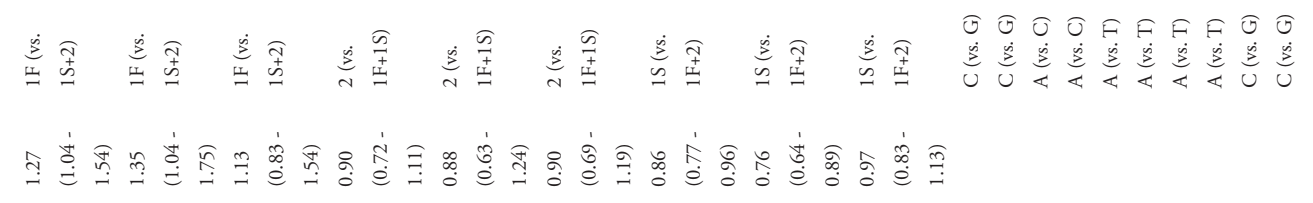

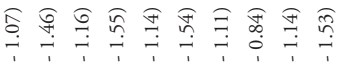
它

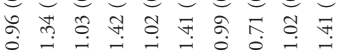

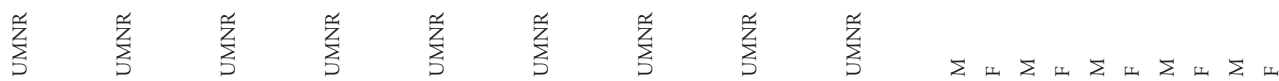

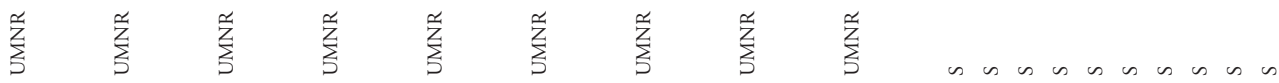

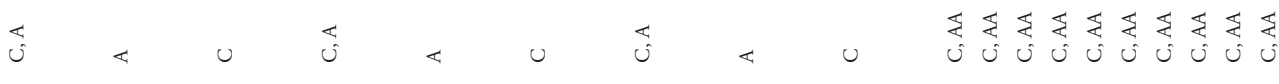

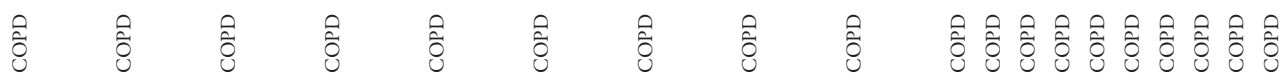

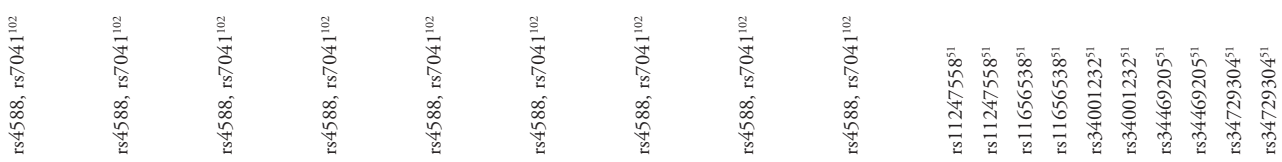

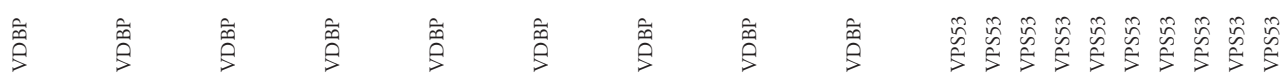


Chapter 3 | Genetic, lifestyle, and environmental risk factors of COPD development

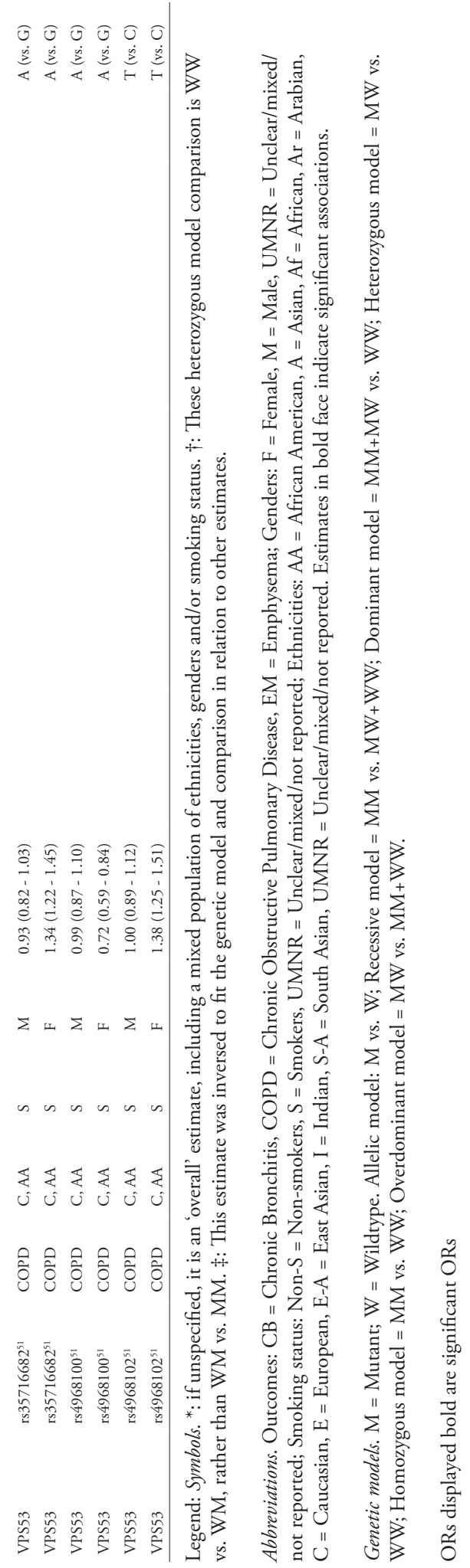


Chapter 3 | Genetic, lifestyle, and environmental risk factors of COPD development

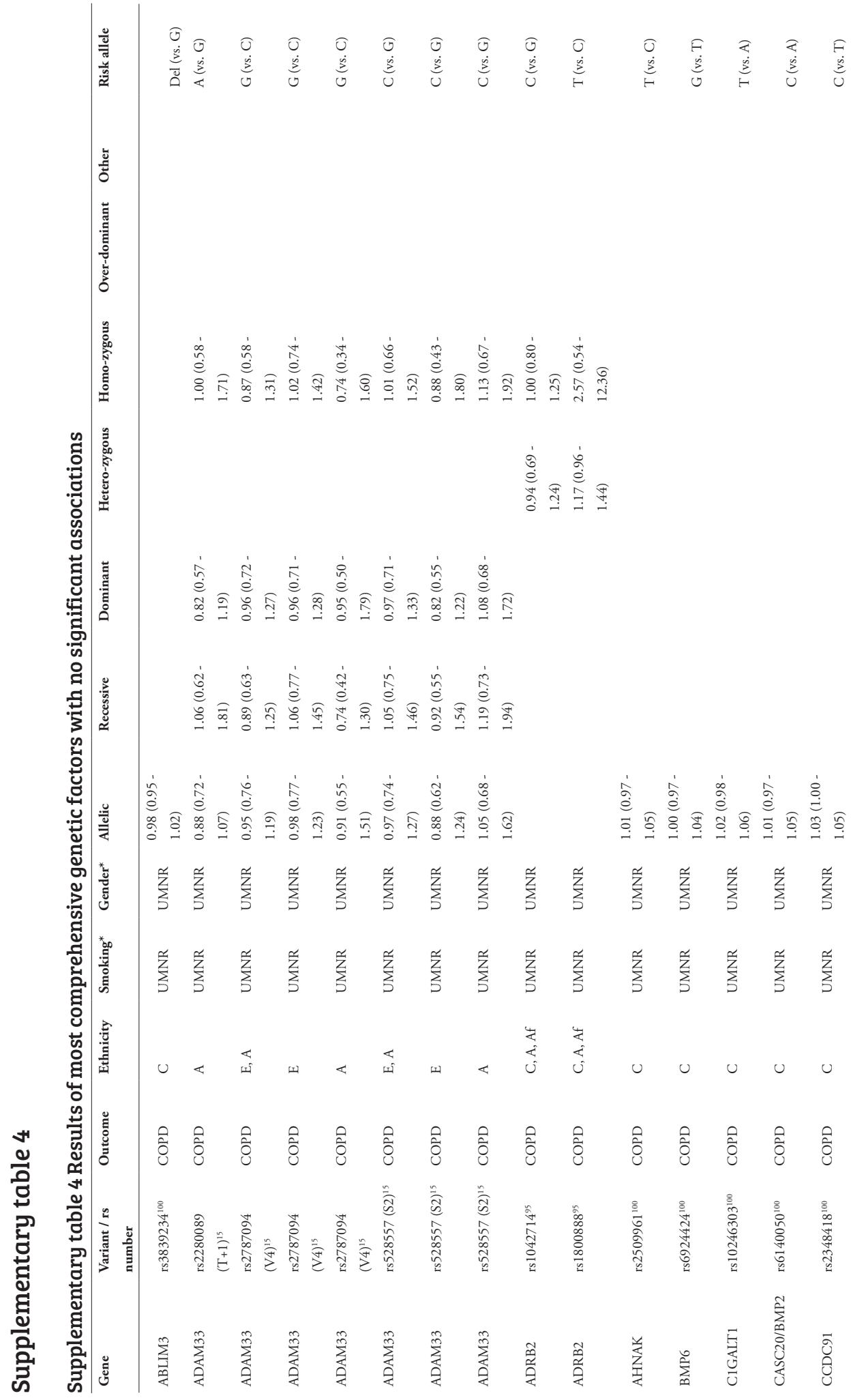


Chapter 3 | Genetic, lifestyle, and environmental risk factors of COPD development

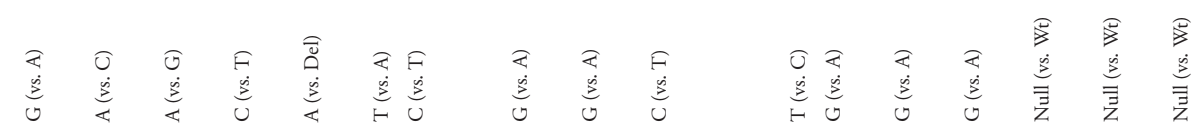

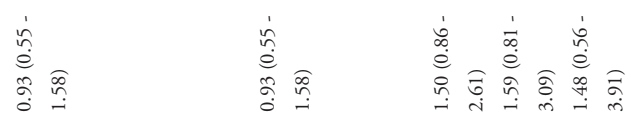

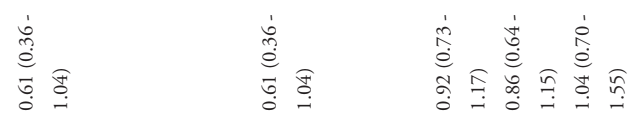

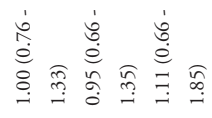

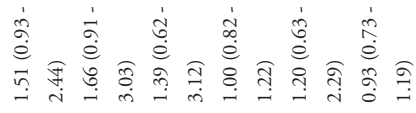

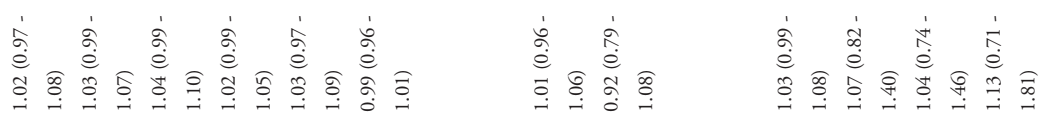

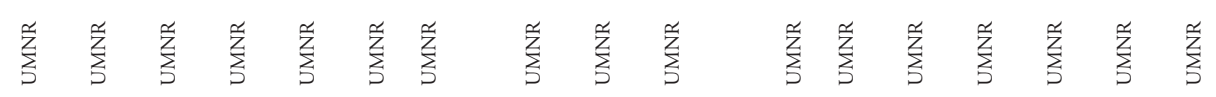

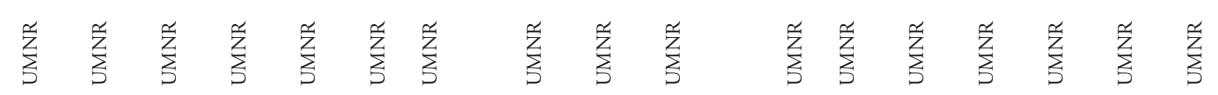

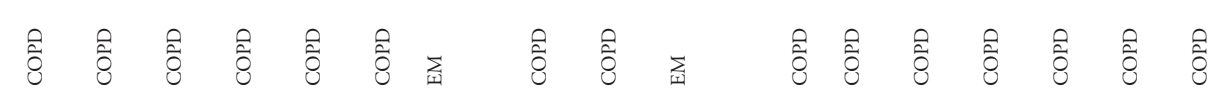

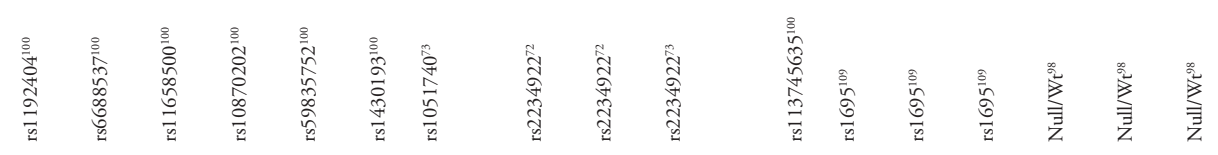

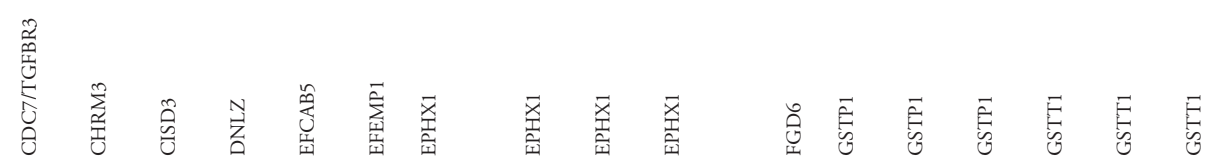


Chapter 3 | Genetic, lifestyle, and environmental risk factors of COPD development

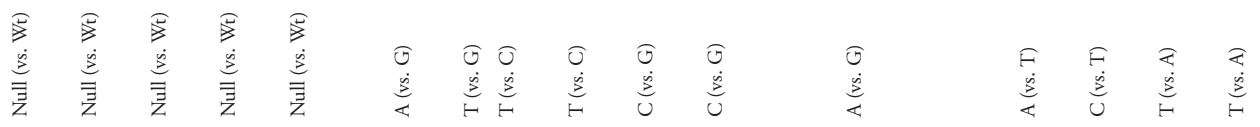

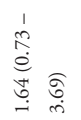

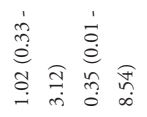

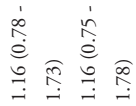

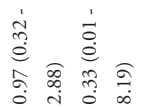

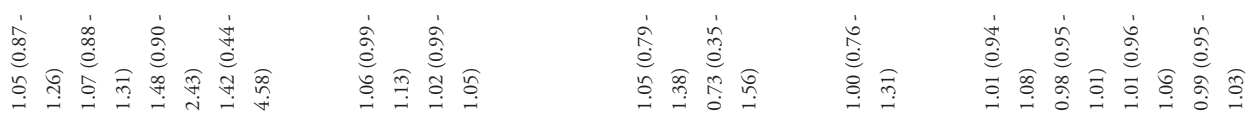

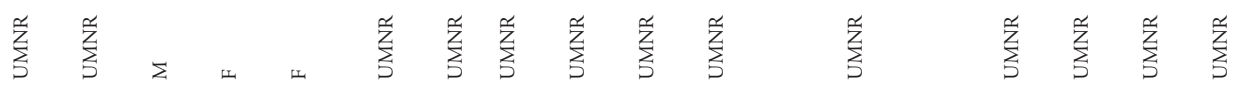

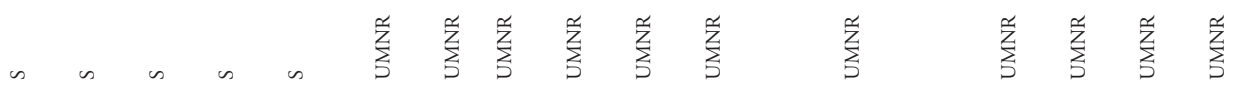

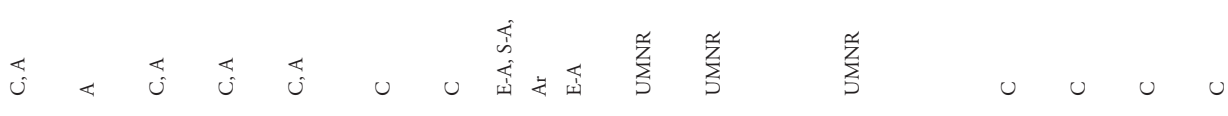

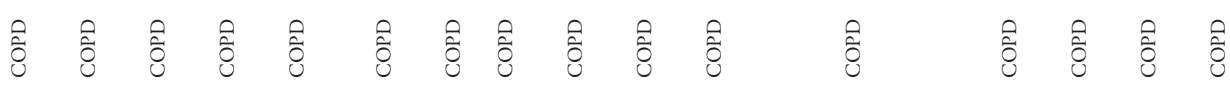

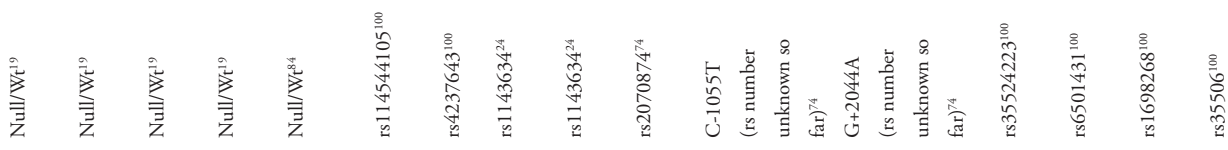

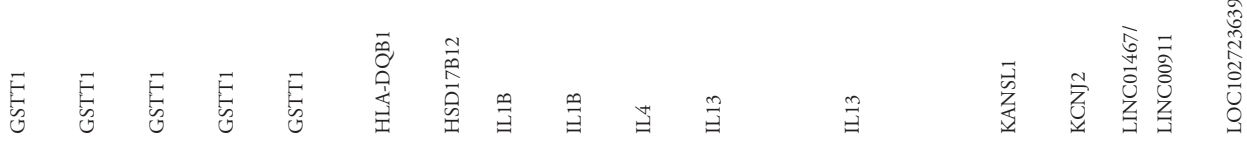


Chapter 3 | Genetic, lifestyle, and environmental risk factors of COPD development

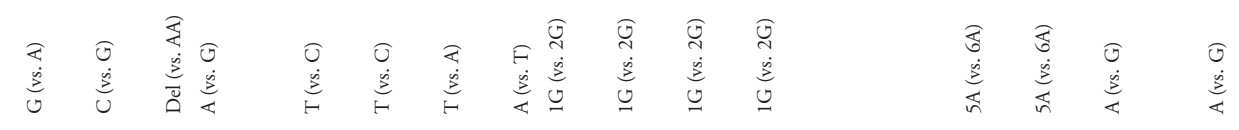
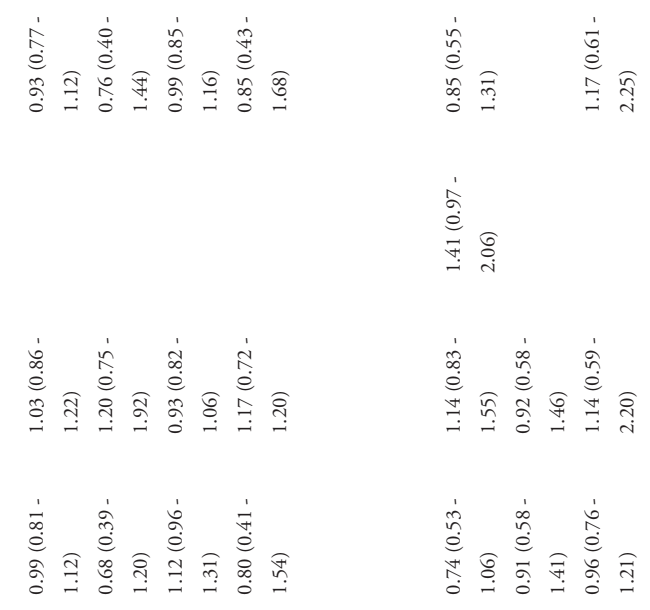

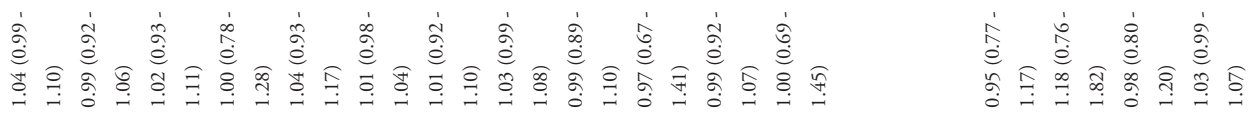

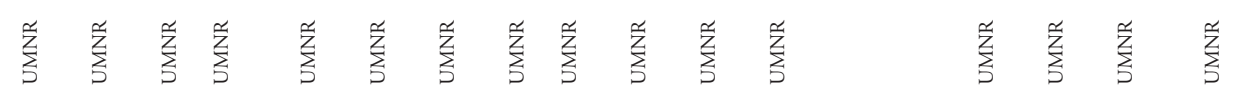

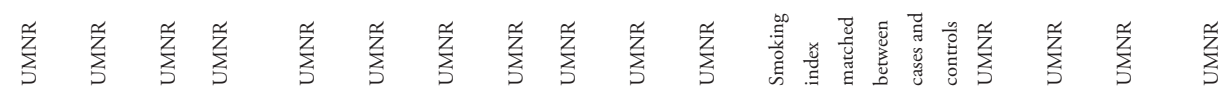
․․ำ ว

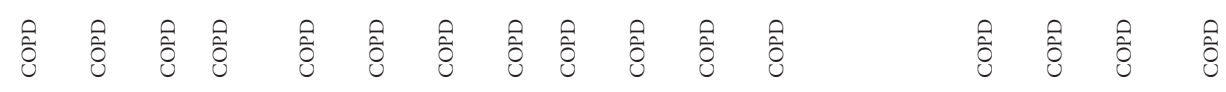

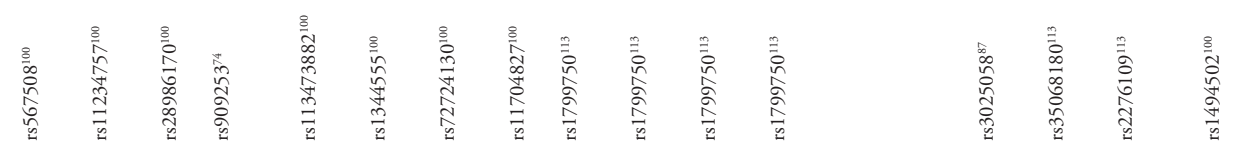

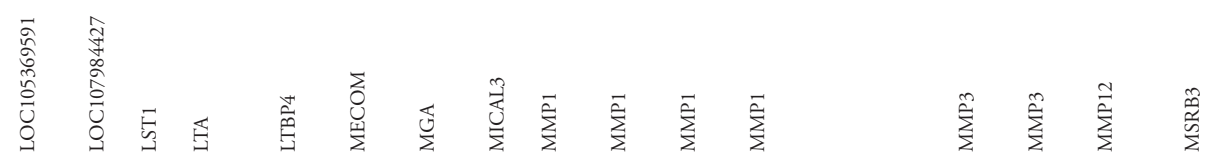


Chapter 3 | Genetic, lifestyle, and environmental risk factors of COPD development

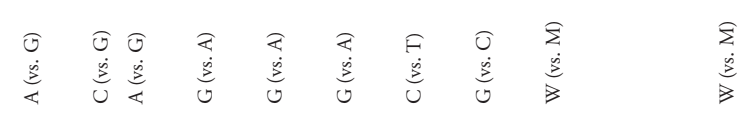

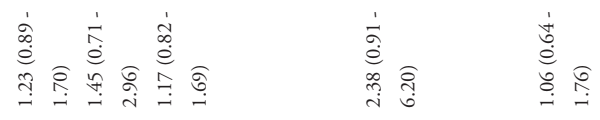

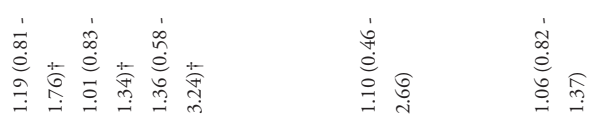

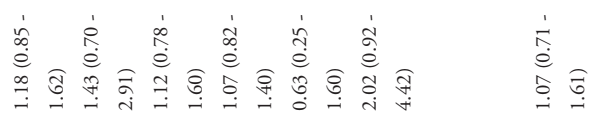

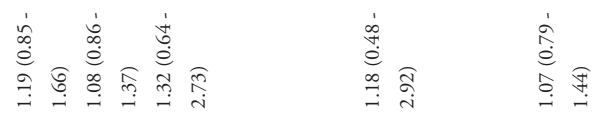

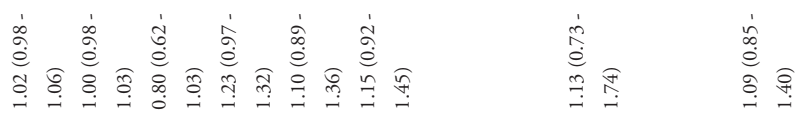

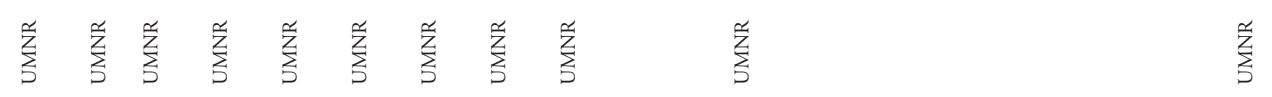

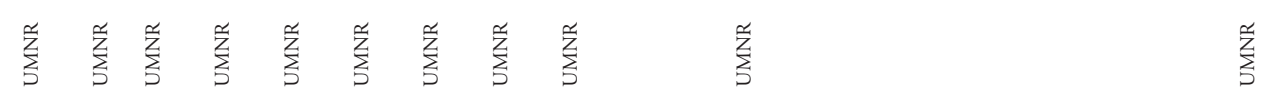

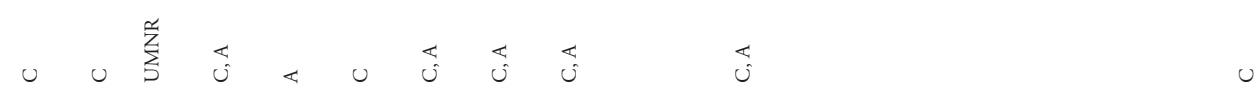

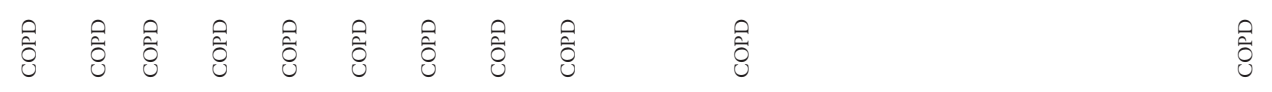

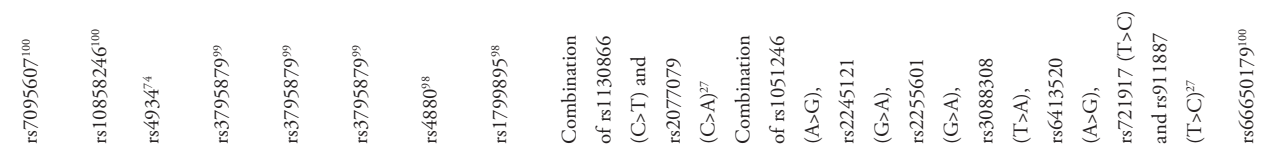

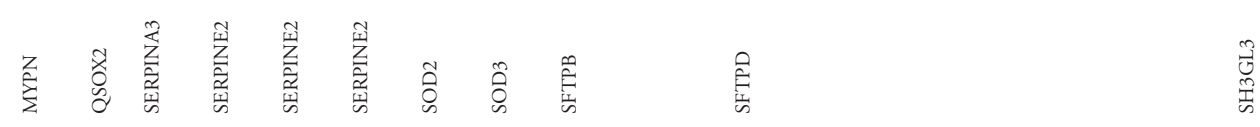


Chapter 3 | Genetic, lifestyle, and environmental risk factors of COPD development

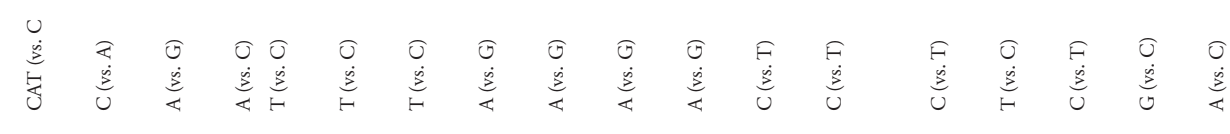

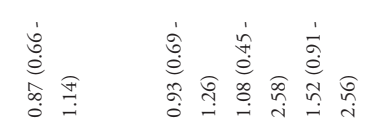

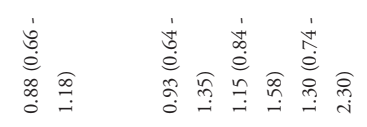

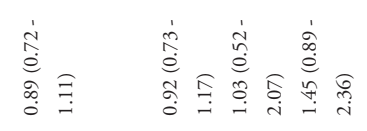

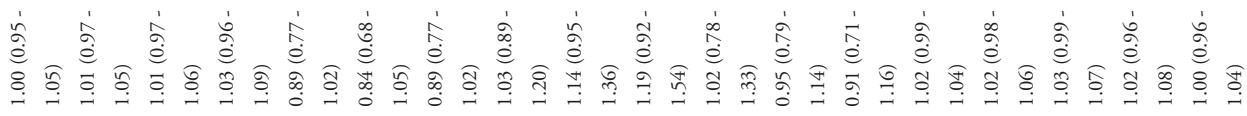

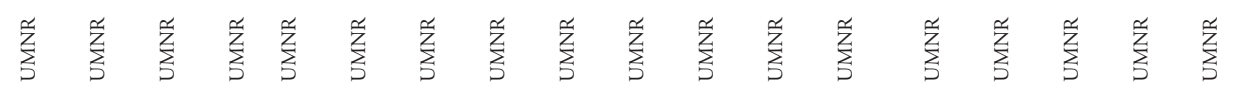

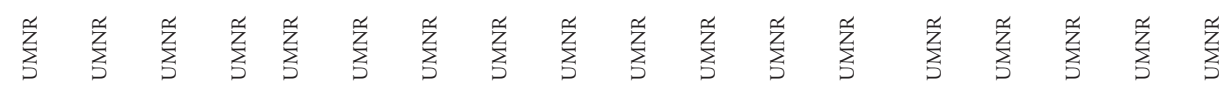

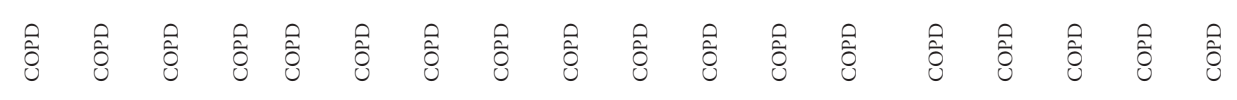

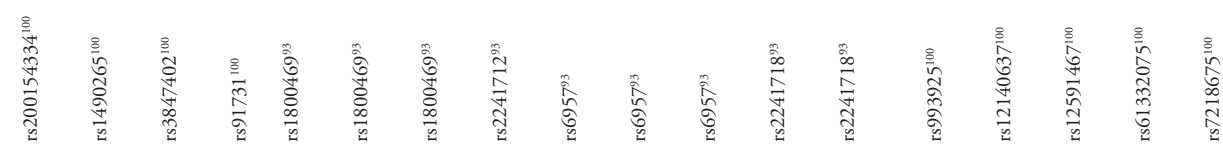

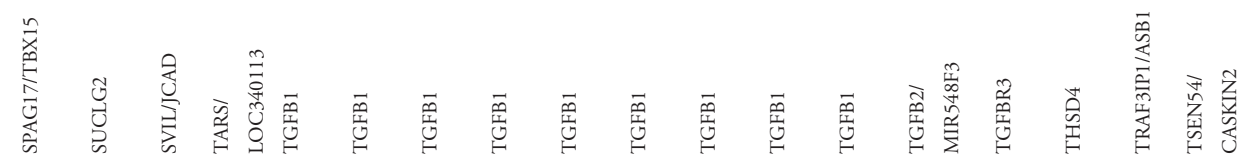


Chapter 3 | Genetic, lifestyle, and environmental risk factors of COPD development

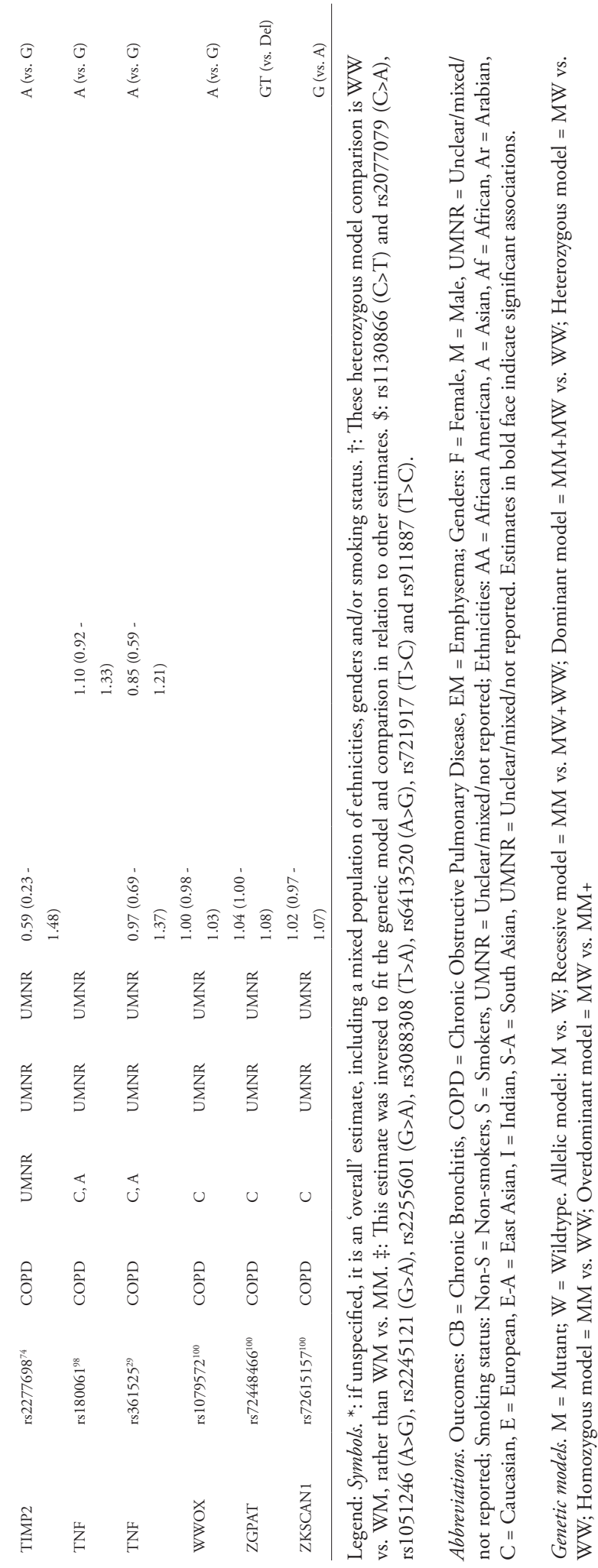


Chapter 3 | Genetic, lifestyle, and environmental risk factors of COPD development 


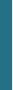




\section{Chapter 4}

Behavioural changes, sharing behaviour and psychological responses after receiving direct-to-consumer genetic test results: a systematic review and meta-analysis

Kelly F. J. Stewart, Anke Wesselius, Maartje A. C. Schreurs, Annemie M. W. J. Schols, Maurice P. Zeegers 


\section{Abstract}

Background. It has been hypothesised that direct-to-consumer genetic tests (DTCGT) could stimulate health behaviour change. However, genetic testing may also lead to anxiety and distress, or unnecessarily burden the health care system. The aim is to review and meta-analyse the effects of DTC-GT on (1) behaviour change, (2) psychological response and (3) medical consumption.

Methods. A systematic literature search was performed in three databases, using "direct-to-consumer genetic testing" as key search term. Random effects metaanalyses were performed when at least two comparable outcomes were available.

Results. After selection, 19 articles were included involving 11 unique studies. Seven studies involved actual consumers who paid the retail price, whereas four included participants who received free genetic testing as part of a research trial (nonactual consumers). In meta-analysis, $24 \%$ had a positive lifestyle change. More specifically, improved dietary and exercise practices were both reported by $16 \%$ and $12 \%$ respectively, whereas $19 \%$ quit smoking. $7 \%$ of participants had subsequent preventive checks. $33 \%$ shared their results with any health care professional and $50 \%$ with family and/or friends. Sub-analyses show that behaviour change was more prevalent among non-actual consumers, whereas sharing was more prevalent among actual consumers. Results on psychological responses showed that anxiety, distress and worry were low or absent and that the effect faded with time.

Discussion. DTC-GT has potential to be effective as a health intervention, but the right audience needs to be addressed with tailored follow-up. Research is needed to identify consumers who do and do not change behaviour or experience adverse psychological responses.

PROSPERO registration number: CRD42016037927 


\section{Introduction}

The momentum for direct-to-consumer genetic testing (DTC-GT) for common disease risks is still increasing among scientists, policy makers, media, and the lay public. This is reflected in on going debates about what should be offered, to and by whom, and if and how it should be regulated; all with the purpose of finding the best balance between protection of customers and supporting their autonomy ${ }^{1,2}$.

A key argument in this debate is one of preventive value or clinical utility. It has been hypothesised that DTC-GT tests can serve as tools to stimulate health behaviour change ${ }^{3}$. Primarily, the assumption is that knowledge of an increased disease risk could lead to risk reducing behaviour among individual customers, as found with penetrant gene testing ${ }^{4}$. In addition, personal disease risk information can be used to further tailor lifestyle interventions and target the right populations for both interventions and health monitoring, likely resulting in increased effectiveness and cost-reduction. However, to date studies have shown that behaviour change as a result of genetic testing is not always found on the population-level ${ }^{5-7}$ or that results remain modest ${ }^{8,9}$, with some sub-groups even showing an unhealthy behavioural response to low disease risks ${ }^{8,10}$. In addition, it has been argued that genetic testing could lead to increased anxiety and distress among customers $^{11}$, but this has largely been refuted ${ }^{12}$. Moreover, concerns have been raised about the unnecessarily burdening of the already strained health care system ${ }^{13}$. Because genetic information and risk information are difficult to understand ${ }^{14,15}$, customers may require additional help in interpreting their results correctly if the information provided by testing companies is insufficient. Consumers may then seek help from their health care professional (HCP) to make sense of their results and to follow up with additional testing.

In order to facilitate evidence-based decision-making with regard to implementation of DTC-GT services and the use thereof for clinical purposes, a quantitative summary of the available data is required. To date, this has not yet been performed. Therefore, the aim of the current study is to review and, where possible, meta-analyse the effects of DTC-GT among a general population on (1) health-related behaviour change, (2) psychological responses and (3) medical consumption, as studied in interventional, cohort, case-control or cross-sectional studies.

\section{Methods}

This study was registered in PROSPERO under registration number CRD42016037927. 


\section{Search strategy}

A systematic literature search was performed until January 2017 in three databases: Web of Science, PubMed, and Embase, using "Direct-to-consumer genetic testing" and "Personal genetic testing" as key search terms. In addition, reference lists of and citations to key publications were investigated for eligible studies. No restriction on language or publication date was applied.

\section{Study selection}

First selection was based on title and abstract, after which full texts were reviewed. Studies were included when they researched health-behaviour change, psychological responses or medical consumption as a result of genetic disease risk testing delivered direct-to-consumers. Original studies, including trials, longitudinal and cross-sectional studies, were included while case studies or commentaries were excluded. Studies were restricted to those studying a general population of adults. Because of the focus on multiplex genetic testing, nutrigenetic, pharmacogenetic or sport-related genetic tests were excluded, as well as genetic testing for highly penetrant genes and prenatal or neonatal genetic tests. Both actual consumers, meaning people having purchased the test by themselves, and non-actual consumers, meaning people having obtained the test through a scientific study, were included. Hypothetical consumers, meaning people receiving mock test results, were excluded.

\section{Data extraction}

All data were extracted by KS and checked for consistency by MS. Disagreement was solved through discussion until consensus was reached. Extracted data included: author, year, title, study design, country of authors, country of study participants, study name (if applicable), actual or non-actual consumer, cost of test, follow-up duration, total participants, and per outcome: the outcome itself (e.g. healthier diet), and size and unit of the outcome (e.g. 13\% changed). Not one specific outcome measure was preferred; acceptable outcome measures included percentages and scores on a validated measurement scale. All data were extracted and cross-checked for comparability.

\section{Selection of data for meta-analysis}

Extracted data were cross-checked for outcomes that were suitable to be combined in meta-analysis, i.e. when outcomes reported the same or similar behaviours with the same unit of measurement. In case of possible overlap between estimates, only the most comprehensive outcome was included for that particular meta-analysis. For example, percentage of people sharing results with 'any health care professional (HCP)' was selected rather than both estimates on 'genetic specialist' and 'general practitioner' separately in the overall analysis on sharing behaviour. If one study reported more than 
one outcome, each estimate was included separately in the relevant meta-analysis.

\section{Data analysis}

Due to the relatively young field of research, it was decided that meta-analysis was acceptable when a minimum of two estimates were available for a comparable outcome parameter. A random-effects meta-analysis was performed using the metaprop command of STATA V14.0 software ${ }^{16,17}$, and confidence intervals were truncated at $0 \%$ or $100 \%$ respectively. Heterogeneity was assessed using the $\mathrm{I}^{2}$-statistic ${ }^{18}$ and explored by doing sub-analyses on whether the test was offered to the participant free or paid and on whether it involved actual consumers or not. Publication bias was evaluated with Begg's adjusted rank correlation test ${ }^{19}$ and Egger's regression asymmetry test ${ }^{20}$.

\section{Results}

\section{Literature search}

A total of 1,315 publications were identified from the database search and through cross-referencing. After removing 592 duplicates, 723 publications remained for first selection based on title and abstract. Of these, 671 publications were removed based on title and abstract, leaving 52 publications. Of these, 19 publications were included based on full text. Reasons for exclusion were: not being an original study, not reporting on desired outcomes, anticipated effect or hypothetical scenarios, no results reported for consumers only, or being an abstract only. The literature search is depicted in the flow chart in Figure 1.

\section{Study characteristics}

The characteristics of the 19 articles, involving 11 unique studies (see Table 1, column 9 for details), included in the systematic review can be found in Table 1. Six studies had a cross-sectional design $n^{9,10,21-24}$, three were longitudinal observational studies ${ }^{5,25-33}$ and two were intervention studies ${ }^{34,35}$. The number of participants ranged from 60 to 2,037 , totalling 6,672 unique participants. Six unique study populations $s^{9,10,22-24,27,30}$ involved actual consumers who paid the full or a reduced retail price for the product. The remaining five studies $5,21,25,26,28,29,31,32,34,35$ included participants who were offered genetic testing for free as part of a research trial (henceforth referred to as non-actual consumers). In general, the studies involved mostly 
Chapter 4 | Behavioural changes, sharing behaviour and psychological responses after DTC-GT

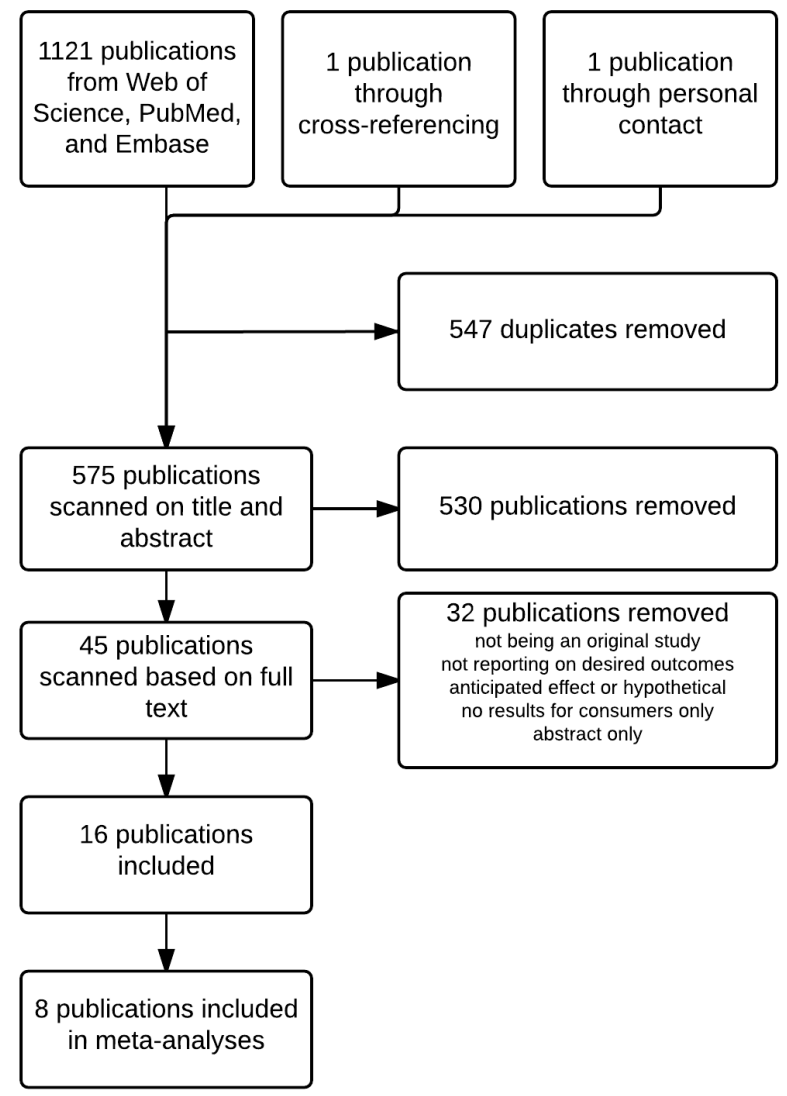

Figure 1 Flow chart of literature search 


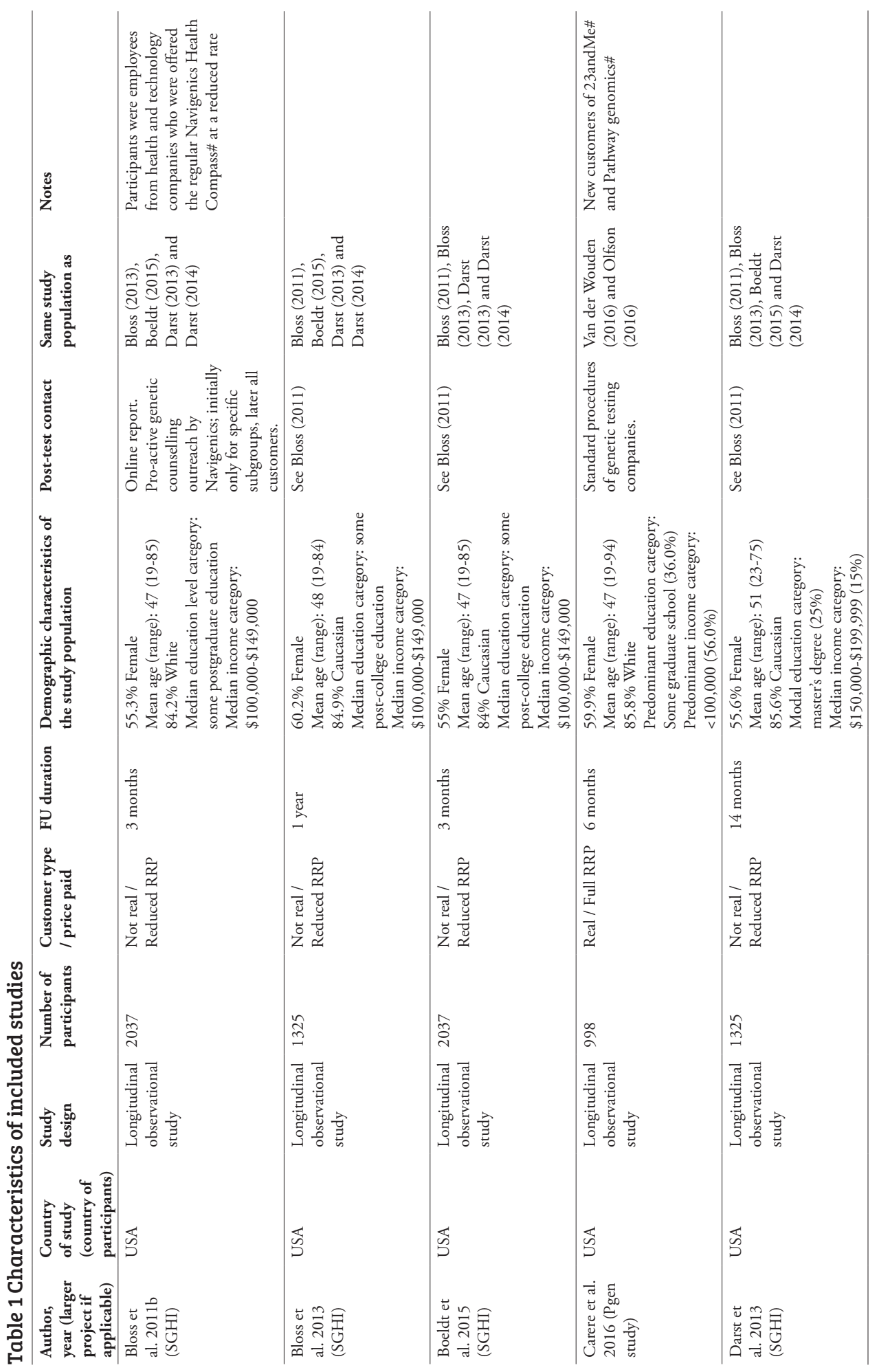




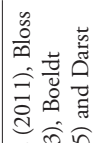

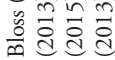

ق.

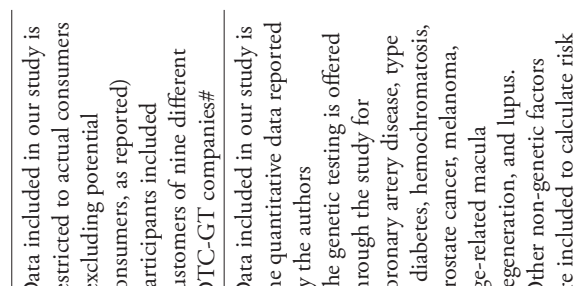

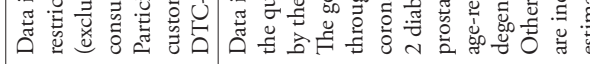

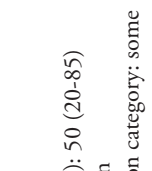

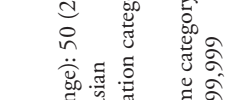

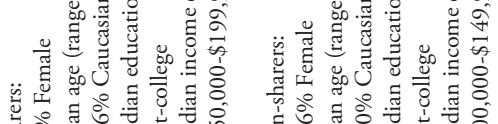

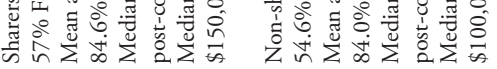

.

量

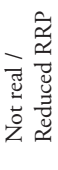

ปั่

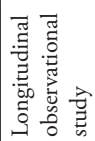

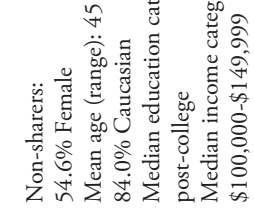

西

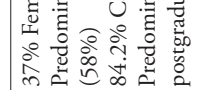

品

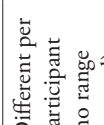

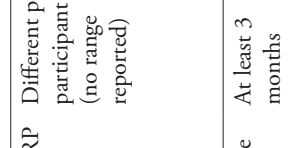

空

$\overleftrightarrow{5}$

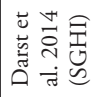

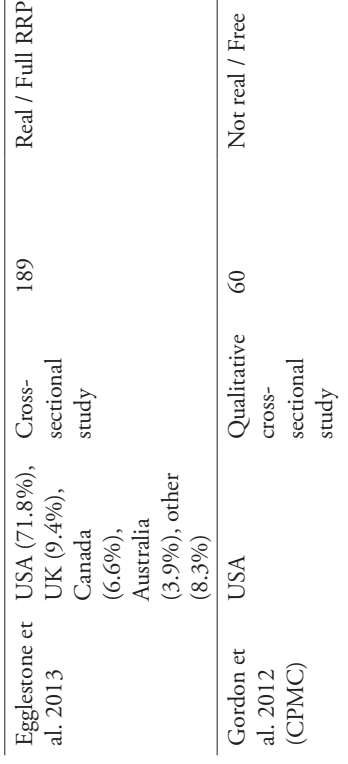


Chapter 4 | Behavioural changes, sharing behaviour and psychological responses after DTC-GT

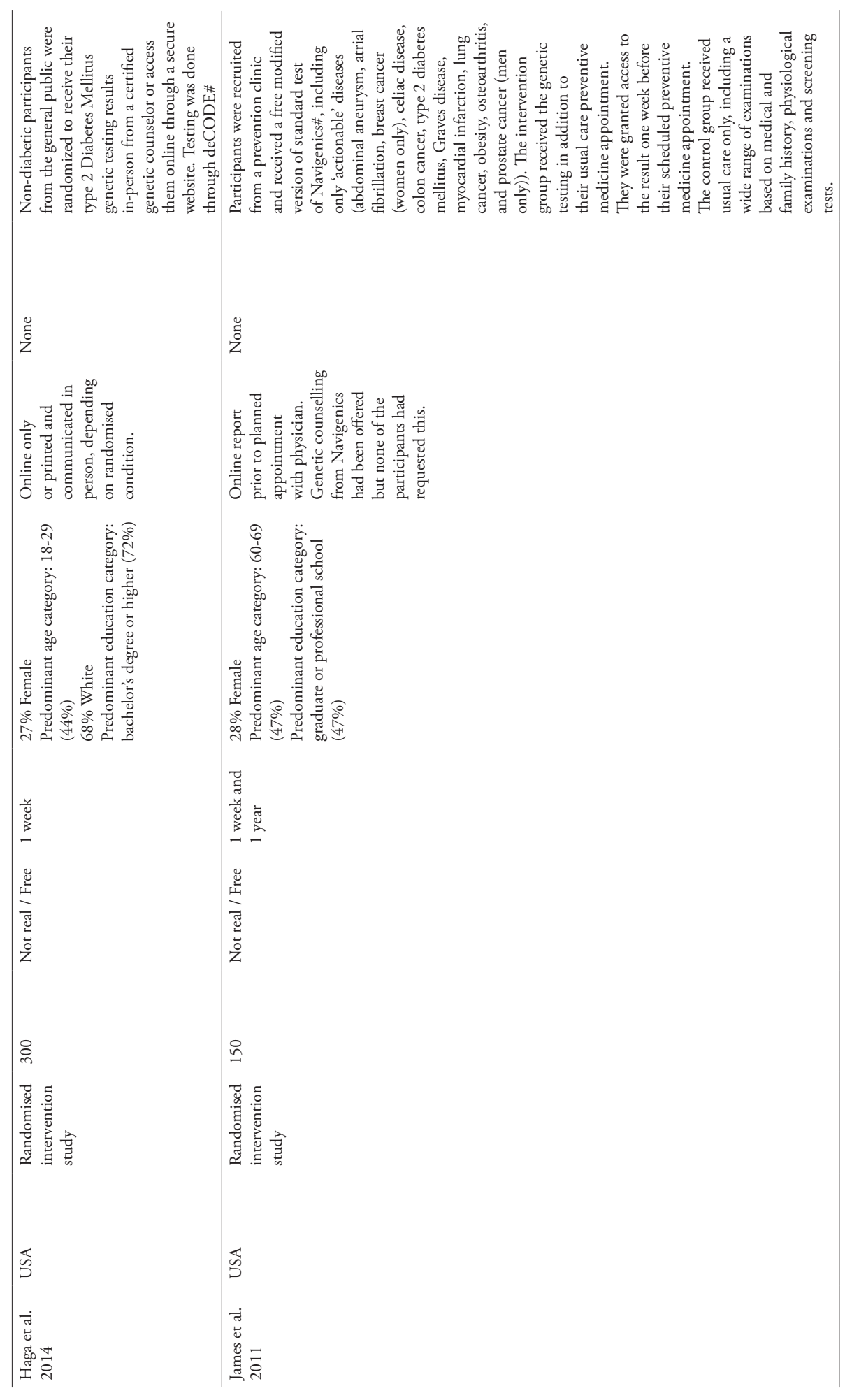


Chapter 4 | Behavioural changes, sharing behaviour and psychological responses after DTC-GT

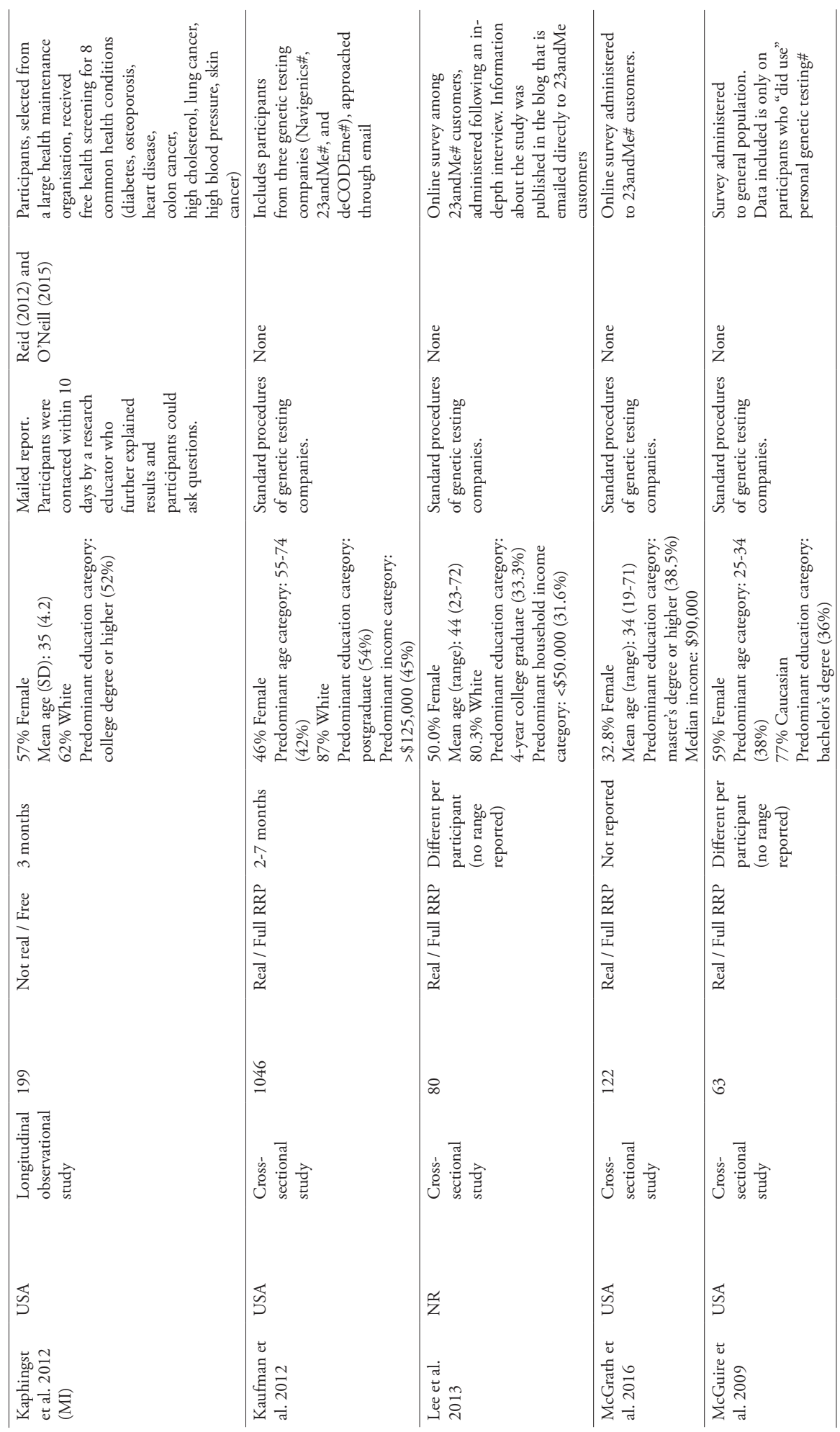


Chapter 4 | Behavioural changes, sharing behaviour and psychological responses after DTC-GT

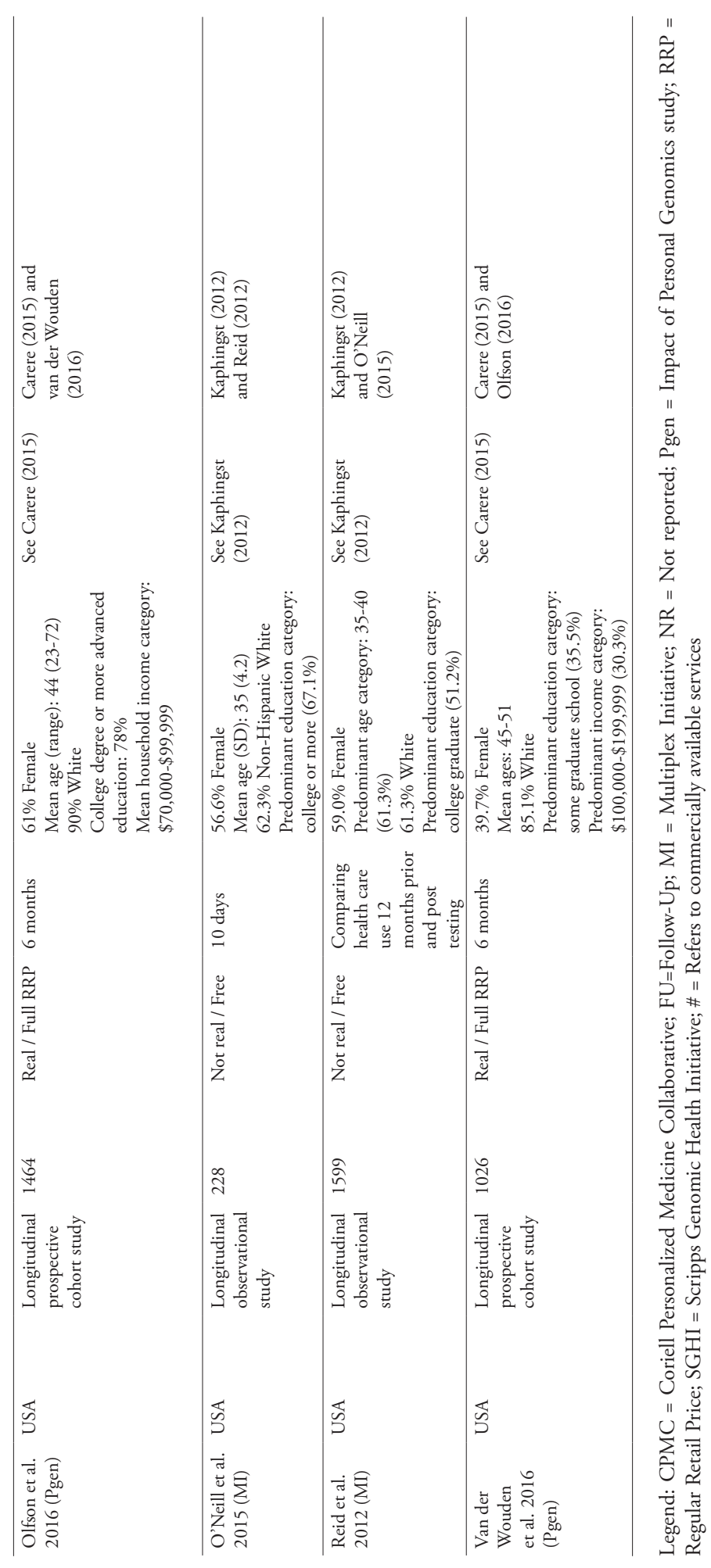


Caucasian individuals with relatively higher education and income levels. Six studies did not alter the standard procedures of the genetic testing companies and offered no additional counselling as part of the study, 10, 22-24, 27, 30, 36. Specific details of what the standard procedures entailed were not clearly provided, but were likely to have included an online report with online educational materials. All studies had online reports, except for one study arm of Haga et al. in which the online report was printed and communicated in person by a genetic counsellor ${ }^{34}$. Four studies included counselling from a HCP (genetic counsellor or physician), either to explain the results at delivery ${ }^{34}$ or after the participant had viewed their results privately, 25, 26, 28, 29, 31-33, 35. One study specifically mentioned to have offered additional online education ${ }^{21}$. Follow-up durations ranged from one week to one year, and four studies, $22-24$ interviewed all participants at one moment, resulting in different follow-up durations per participant.

Seven articles $5,9,10,21,25,26,31$ reported on behaviour changes, eight articles $5,9,25-27,31,34,35$ on psychological effects and fifteen articles, $5,10,21-32$ on sharing results with others and medical follow-up. Estimates from 11 articles 5 , 9, 10, 21-25, 27, 30, 31 were included in at least one meta-analysis.

\section{Health-related behaviour change}

Table 2 shows the results with regard to behaviour change. A wide range of lifestyle behaviours have been studied, including general dietary practices, fat intake, caffeine intake, vitamin and supplement use, weight loss, alcohol use, smoking, and exercise behaviour. All studies reported results as the percentage of people with a certain changed behaviour ${ }^{9}, 10,21,25,36$, and one study ${ }^{5,25}$ also measured actual behaviour through questionnaires. All of these were self-reported. Any positive lifestyle change ranged from $14 \%$ for exercise behaviour ${ }^{10}$ to $33 \%$ for dietary behaviour ${ }^{10}$ or any lifestyle change $^{21}$. Two studies ${ }^{25,36}$ found that between $18 \%$ and $22 \%$ of pre-test smokers had quit smoking. Between $3.2 \%$ and $20.5 \%$ of people undergoing genetic testing made a change in their vitamin supplementation ${ }^{9}, 10,25$. Information-seeking behaviour varied widely, which may also depend on the type of information sought: $1.6 \%$ looked up information on their high-risk items ${ }^{9}$, whereas $65 \%$ had looked up information about how health habits influenced their risk of disease ${ }^{31}$. The results from the exercise and dietary fat intake questionnaires showed no change for either outcome compared to pre-testing ${ }^{5,25}$. Behaviour change was not mediated by perceived control of the disease or the actual genetic risk that was received ${ }^{26}$. 


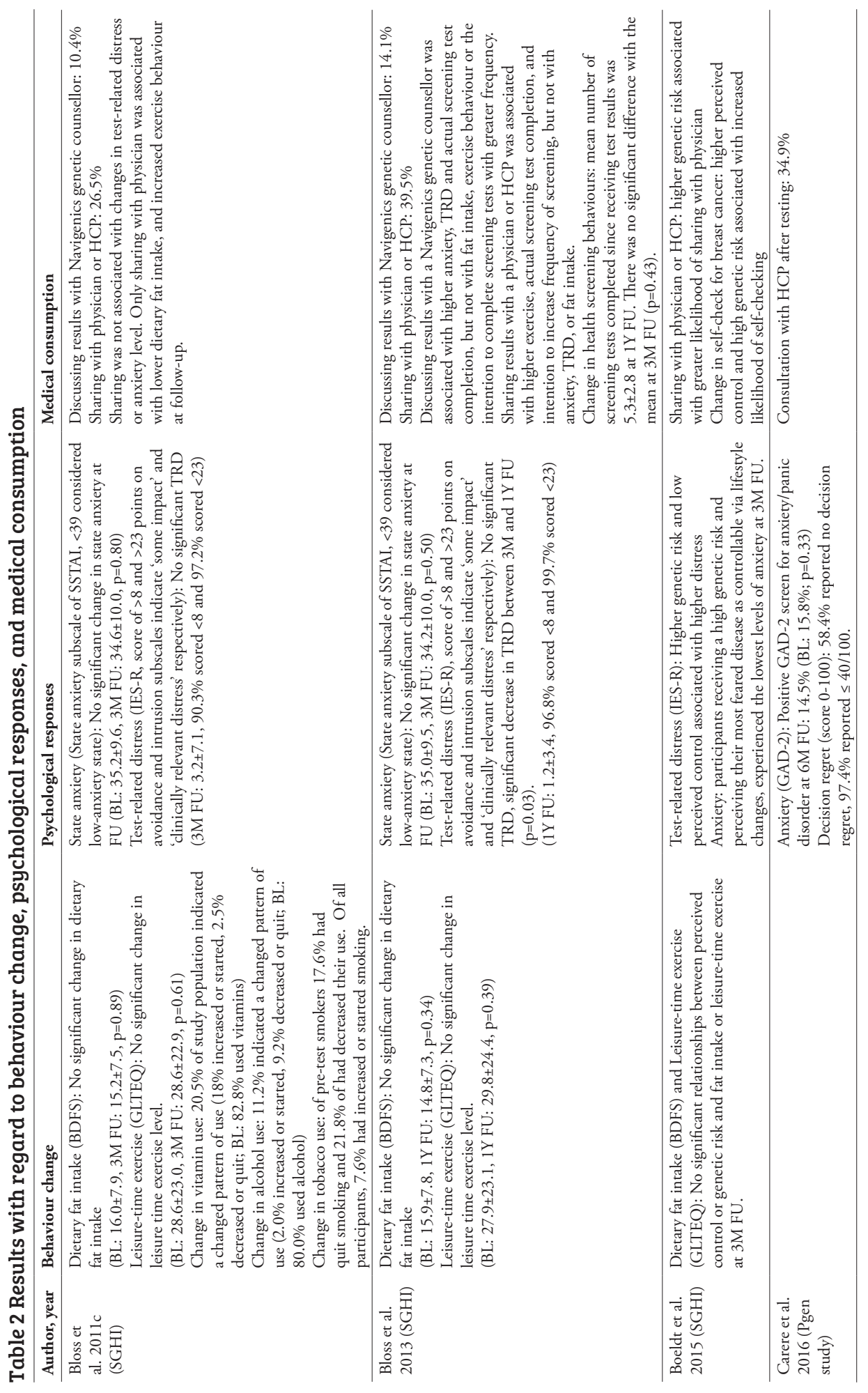


Chapter 4 | Behavioural changes, sharing behaviour and psychological responses after DTC-GT

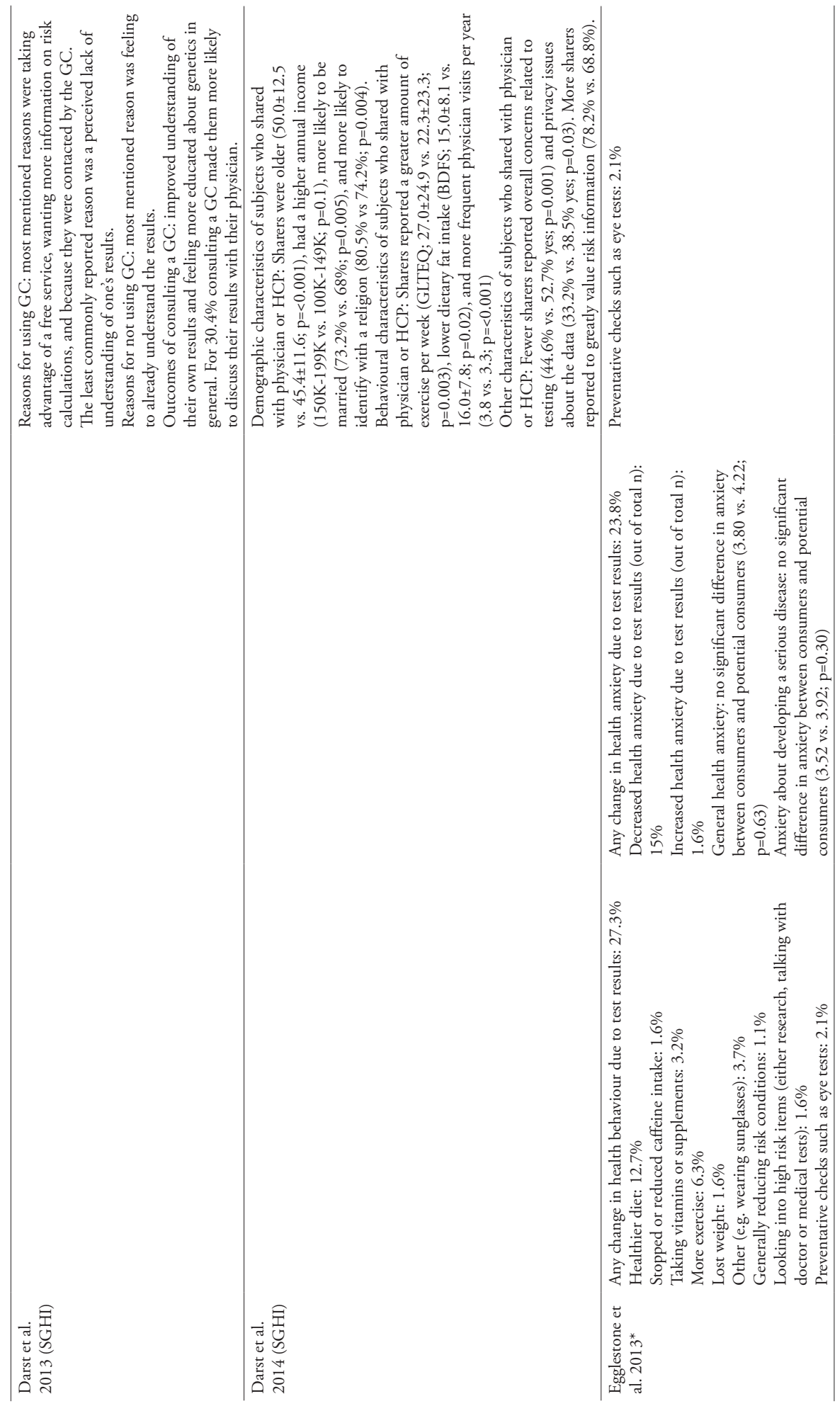


Chapter 4 | Behavioural changes, sharing behaviour and psychological responses after DTC-GT

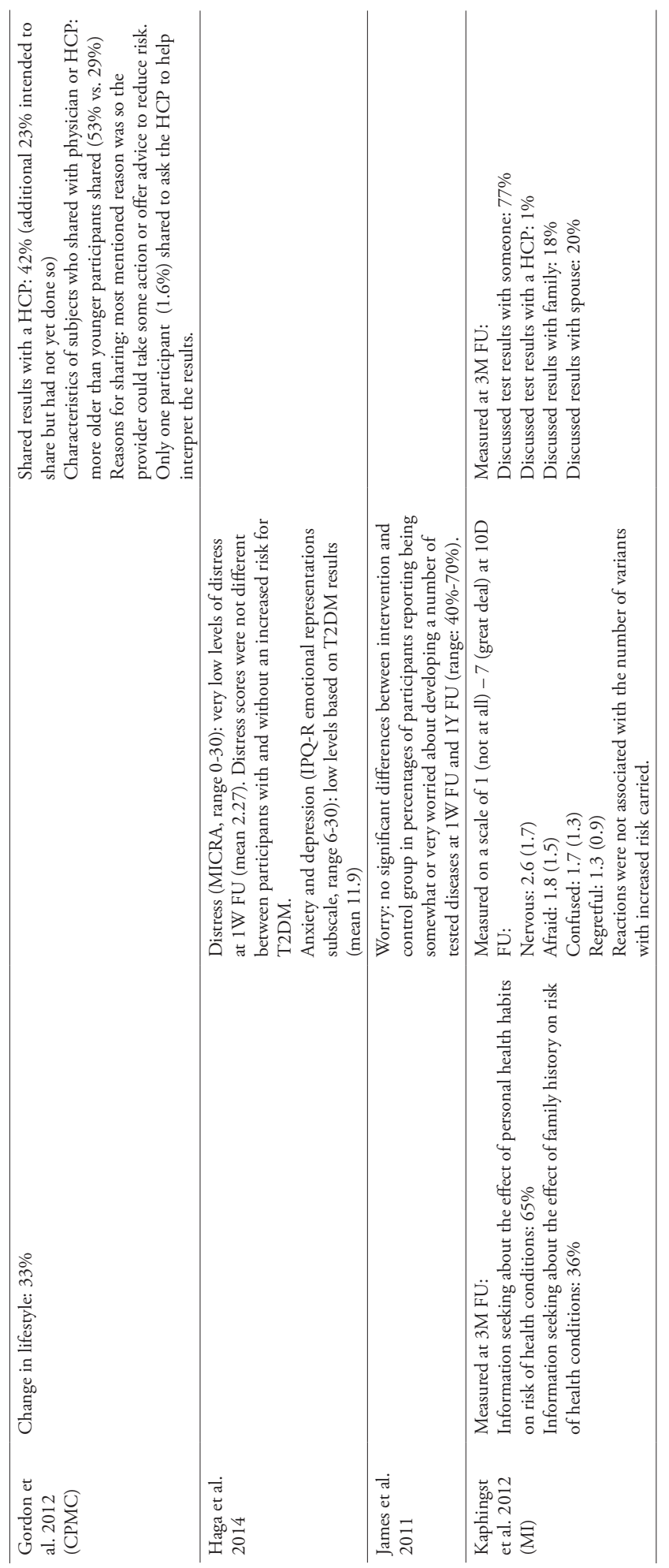




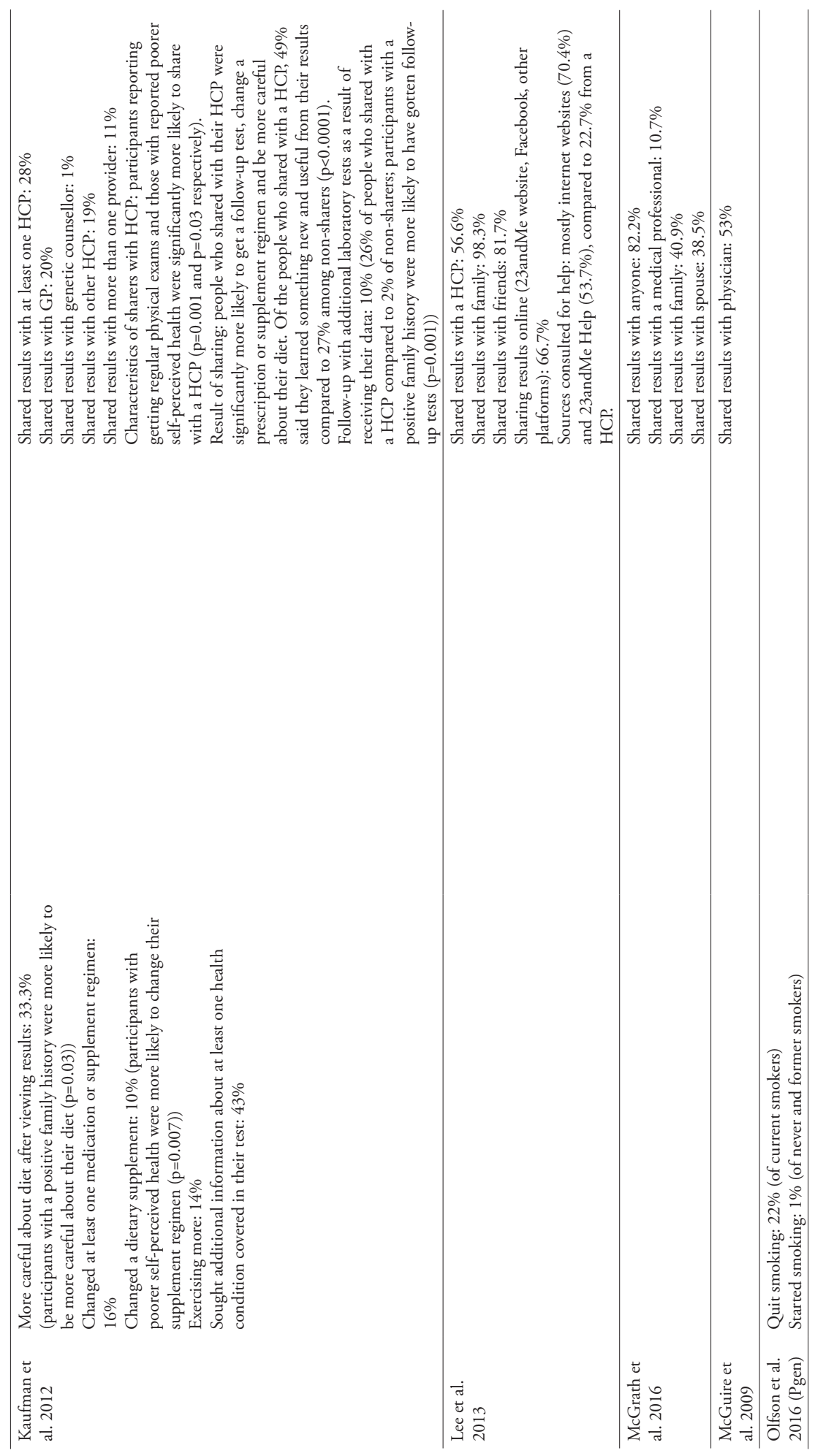


Chapter 4 | Behavioural changes, sharing behaviour and psychological responses after DTC-GT

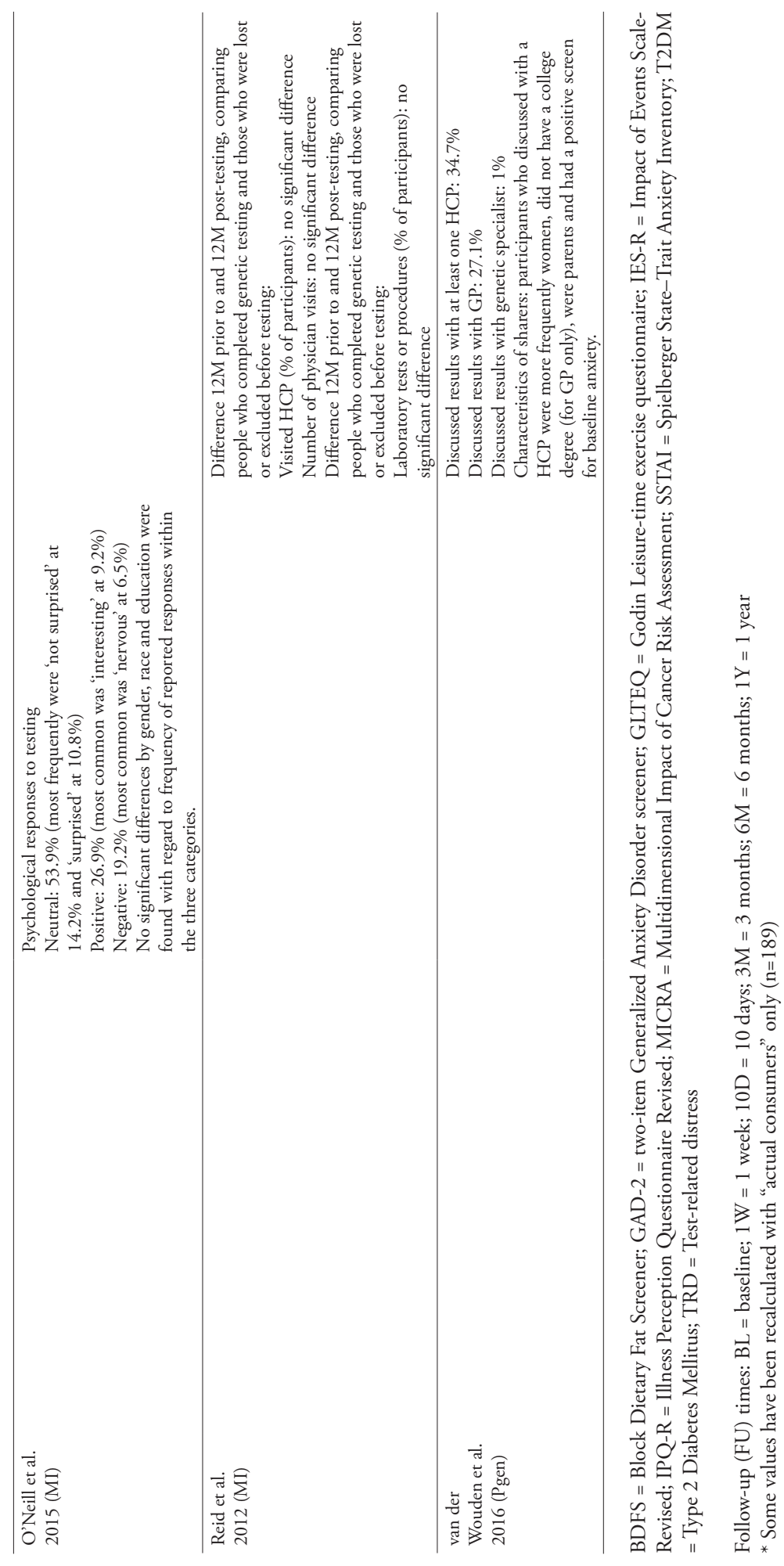




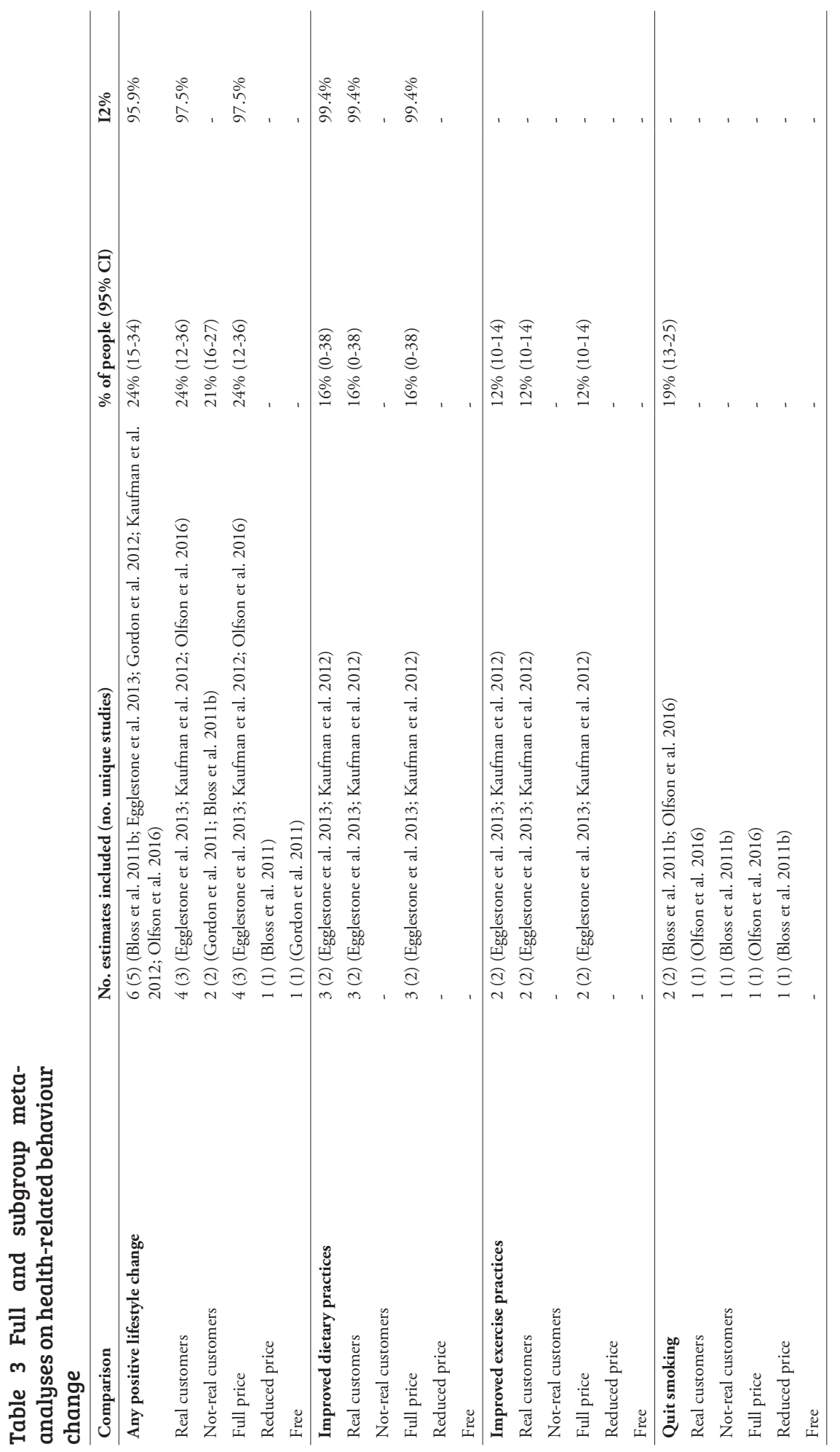


Chapter 4 | Behavioural changes, sharing behaviour and psychological responses after DTC-GT

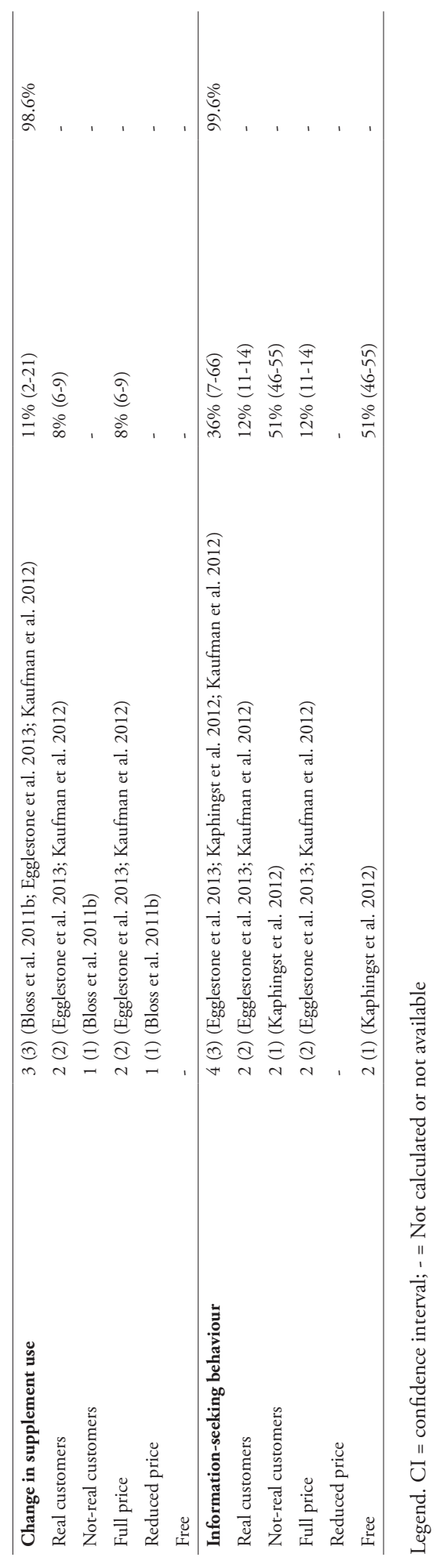




\section{Meta-analyses for health-related behaviour change}

Outcome parameters that were considered suitable for meta-analysis were the percentages of people with (1) any positive lifestyle change, (2) improved dietary practices, (3) improved exercise practices, (4) quitting smoking, (5) changed supplementation use, and (6) information-seeking behaviours. The results are displayed in Table 3 and forest plots can be found on Online resource 1. The overall proportion of people showing any positive lifestyle change after DTC-GT was 24\% (95\%CI: 15-34). For this estimate, no publication bias was detected with either Begg's $(\mathrm{P}=0.573)$ or Egger's test $(\mathrm{p}=0.571)$. More specifically, improved dietary practices was reported by 16\% (95\%CI: 0-38), improved exercise practices also by $12 \%$ (95\%CI: 10-14), quitting smoking by $19 \%$ of pre-test smokers (95\%CI: $13-25)$ and change in supplementation use by $11 \%(95 \% \mathrm{CI}$ : 2-21). More than a third of participants $(36 \%, 95 \%$ CI: 7-66) had sought information, including information-seeking for the disease itself, healthier lifestyle and heritability.

Results of the subgroup analyses show similar levels of any positive lifestyle change among actual consumers and participants recruited through trials (24\% and 21\% respectively). Information seeking behaviour was more prevalent among participants recruited in trials receiving a free test $(51 \%$, compared to $12 \%$ of actual consumers paying the full price). Subgroup analyses on the other outcomes were not possible. It should be noted that only one study of non-actual consumers reported on positive lifestyle changes. Psychological responses

Table 2 shows the results of all findings with regard to psychological responses. In general, the effect of testing on a number of psychological responses, including anxiety, distress, and worry, was low or absent $5,9,25,27,31,33,34$ and this effect faded with time ${ }^{5}$. Increased perceived control led to lower levels of anxiety and distress in one study ${ }^{26}$. One study showed higher levels of distress with increased genetic risk ${ }^{26}$, whereas another study found no difference ${ }^{34}$. Two studies that reported on regret of testing ${ }^{27,31}$ found low levels of regret. Gender, race and education were not associated with frequency of reported positive, neutral or negative responses ${ }^{33}$.

The outcome parameters between the studies were considered too heterogeneous for meta-analysis, so no meta-analysis was performed.

\section{Medical consumption}

Table 2 shows the results of all findings with regard to any form of sharing behaviour and additional medical follow-up. Most people shared their results with someone, which could be family and friends, online or a HCP. In general, more people shared with their HCP (general practitioner or other) than with a genetic specialist. The percentage of 
participants discussing the results with family or friends ranged widely between the included studies, from around $20 \%$ in one ${ }^{31}$ to $98 \%$ another ${ }^{22}$.

The effect of DTC-GT on preventive screening behaviour was little to none, ${ }^{5,}$, although greater likelihood of self-checking was found with higher perceived control of the disease (through lifestyle changes or medical attention) and higher genetic risk received ${ }^{26}$. However, sharing with a HCP, led to additional follow-up tests ${ }^{10}$ and increased screening behaviour ${ }^{5}$. One study ${ }^{32}$ specifically researched the effect of DTCGT on medical follow-up comparing to a control group, and found no difference in the percentage of people who visited their HCP, the number of visits, and the percentage of people with lab tests or procedures.

\section{Result of sharing}

People who had shared their results with a HCP had lower dietary fat intake and higher exercise at follow-up ${ }^{5,25}$, were more careful about their diet and more often changed a prescription or supplemental regimen ${ }^{10}$. Sharing with a HCP did not lead to lower levels of anxiety or distress in one study at three months post-testing ${ }^{25}$, while sharing with a genetic specialist actually increased anxiety and distress at one year post-testing in the same study5. More HCP sharers than non-sharers indicated that they had learned something new and useful from their genetic tests ${ }^{10}$. Sharing with a genetic specialist improved participant's understanding of own results and made them feel more educated about genetics ${ }^{28}$. About a third of the participants in the same study reported that sharing with a genetic specialist made them more likely to discuss their results with their physician $^{28}$.

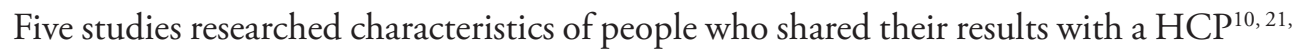
$26,29,30$. In summary, people who shared their results with a HCP were more likely to be women ${ }^{30}$, older $^{21,29}$, married $^{29}$, or parents ${ }^{30}$. They also appeared to have a higher annual income ${ }^{29,30}$, and identified with a religion ${ }^{29}$. They exercised more and had a lower fat intake $^{29}$, more frequently visited their physician ${ }^{10,29}$, had poorer self-perceived health ${ }^{10}$ and more often had a positive screen for anxiety prior to testing ${ }^{30}$. Finally, they also had fewer concerns related to testing and privacy issues regarding the data ${ }^{29}$, greatly value the risk information ${ }^{29}$, and had greater genetic risks ${ }^{26}$.

\section{Meta-analyses for medical consumption}

Outcome parameters that were considered suitable for meta-analysis were the percentages of people who shared their results with (1) any HCP, (2) general practitioners, (3) genetic specialists, and (4) family and/or friends, and the (5) percentage of people undergoing preventative checks. Table 4 shows the results of these meta-analyses. One 


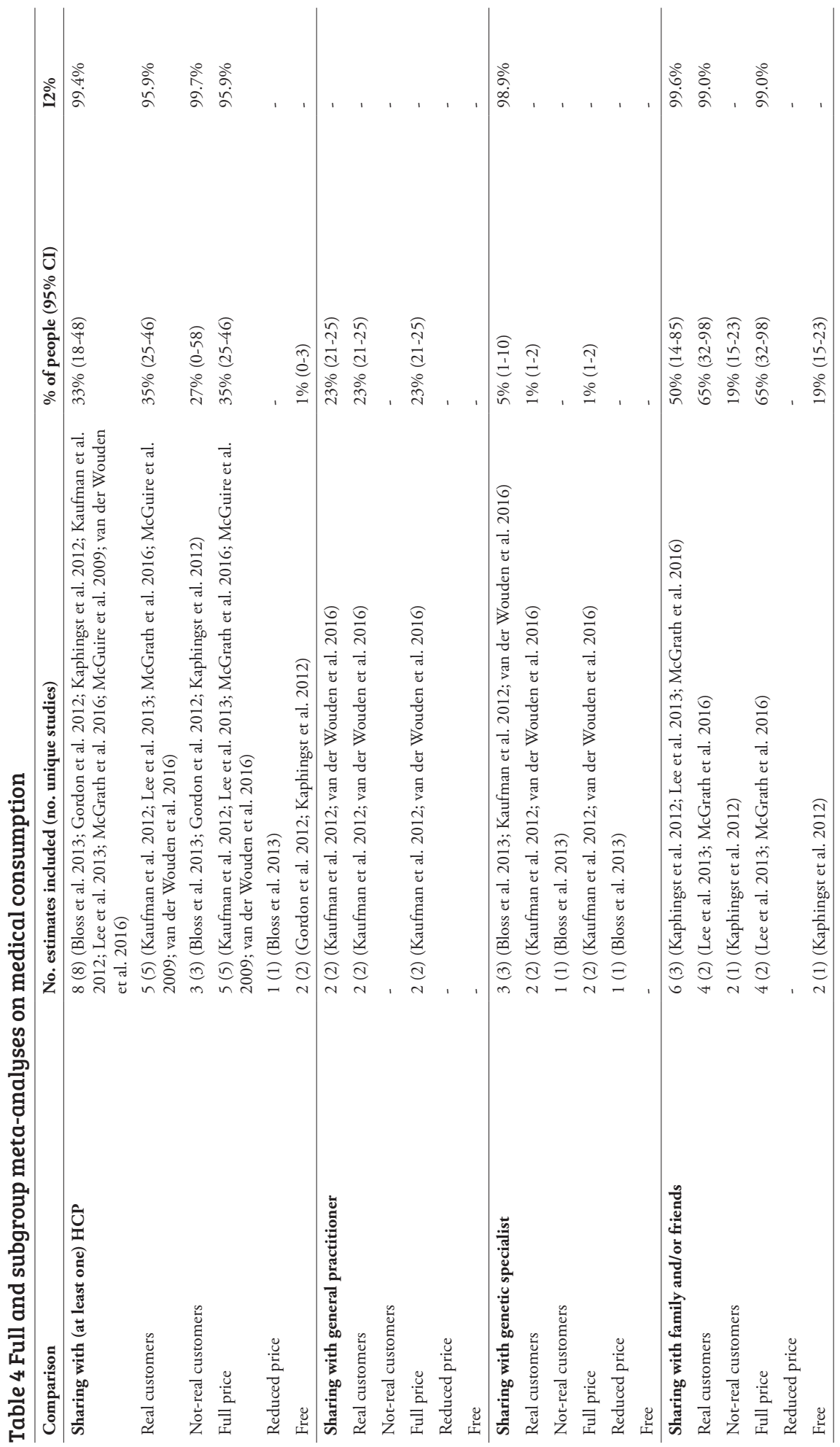


Chapter 4 | Behavioural changes, sharing behaviour and psychological responses after DTC-GT

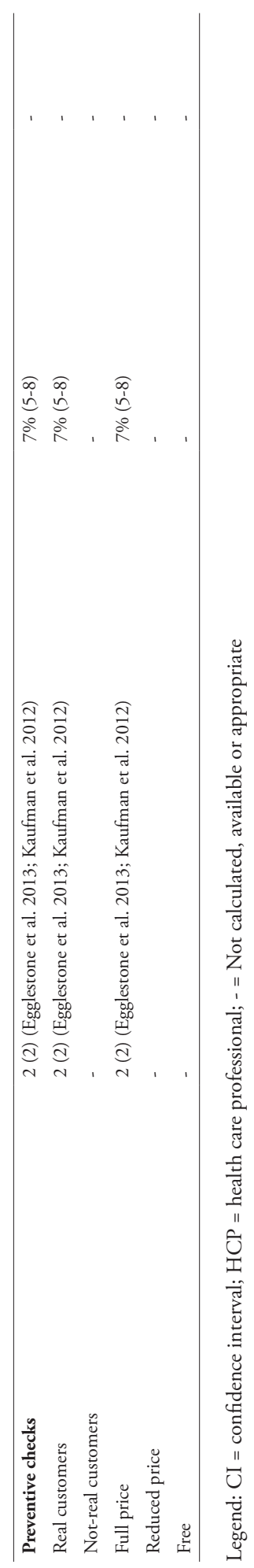


third of participants (33\%, 95\%CI: 18-48) reported to have shared their results with any HCP, among which general practitioners, genetic specialist or other specialists. For this estimate, no publication bias was detected with either Begg's $(\mathrm{P}=0.458)$ or Egger's test $(\mathrm{p}=0.120)$. Of these, $23 \%$ (95\%CI: 21-25) had shared with their general practitioners whereas 5\% (95\%CI: 1-10) had shared with a genetic specialist. Half of participants (50\%, 95\%CI: 14-85) reported to have shared their results with family and/or friends. The percentage of people having preventive checks, including screening practices and laboratory tests, was 7\% (95\%CI: 5-8).

Results of the subgroup analyses show higher percentages of participants who shared their results with (at least one) HCP among customers paying the full price versus those receiving it for free ( $35 \%$ vs. $1 \%)$, and among actual consumers versus nonactual consumers (35\% vs. $27 \%$ ). Participants had shared more often with family and/ or friends among the studies of actual consumers paying full price $(65 \%)$ compared to studies involving non-actual consumers receiving the test for free (19\%). Subgroup analyses for these subgroups on the other outcomes were not possible. It should be noted that both estimates of sharing with family and/or friends come from one study.

\section{Discussion}

\section{Changes in response to testing}

\section{Health-behaviour change}

The results of our meta-analyses show that, when genetic testing is offered direct-toconsumers without additional lifestyle counselling, the effects on behaviour change are modest. Whereas on average, just under a quarter of people reported any health behaviour-related change, little is known about the size of the effect and whether change is maintained at long term. For example, studies asking participants 'whether they changed' found both positive and negative effects, but with more objective measures of behaviour the finding was not replicated ${ }^{5,25,26}$. Therefore, it is paramount that future studies measure behaviour change pre- and post-testing, with validated and more objective measures. Furthermore, although we found that $19 \%$ of smokers quit smoking after undergoing DTC-GT, it is unclear if cessation was maintained at followup. A meta-analysis on genetic testing-based smoking cessation interventions found a significant improvement in cessation rates at short-term follow-up but not at long-term $(\geq 6 \text { months })^{7}$. Finally, sub-analyses show no great difference in 'any positive lifestyle change' between actual consumers paying the full price and non-actual consumers paying a reduced price or receiving the test for free, and therefore large differences in effect for this outcome in normal practice of DTC-GT services need not be expected. 
A possible distortion in the found effects may be due to it being likely that not all participants required change in a specific behaviour, thereby underestimating the relevant impact. In contrast, estimates for quitting smoking were reported for pre-test smokers only, showing how many participants who required changing, had changed the behaviour. Because it is unclear how many participants required a specific behaviour change, the effects may be greater when focussing only on those requiring change.

\section{Undesired consequences of testing}

Typical concerns of opponents of DTC-GT are potential adverse psychological responses, such as anxiety or test-related distress, and inappropriate responses to testing, such as foregoing screening when receiving reduced risks or taking unnecessary or inappropriate preventive measures, including the change of prescription medication. The results of our study show that there is currently little to no evidence for serious adverse psychological responses among consumers. In addition, only a small percentage of people show potentially inappropriate responses, such as changing prescription and over-the-counter medication ${ }^{10}$. However, the genetic testing results in the study in which this was found also included some pharmacogenomic testing, which may explain and possibly justify the medication change by consumers, provided that the pharmacogenomic test results were understood correctly. Similarly, it is not necessarily true that starting a supplementation regimen is a positive lifestyle change ${ }^{37}$. Although the percentages of people with adverse responses or reactions are low, the consequences when it does happen may still be significant. These are delicate issues, which should receive attention from both genetic testing companies and HCPs.

\section{Sharing behaviour}

We found that a third of participants shared their results with at least one HCP. This often led to additional follow-up tests or health screening ${ }^{10,25}$. Whereas in some cases this will result in early detection and prevention (e.g. participant \#11, in ${ }^{21}$ ), in other cases additional tests may be done merely to reassure the individual, unnecessarily burdening the health care system. It has been estimated that the highest downstream costs may range from $\$ 40$ to $\$ 20.604^{38}$.

A significant role remains for genetic testing companies to put great effort in communication of results and aid their customers in correct interpretation of their findings without help of any health care professionals. The study that reported only $1 \%$ of people contacting a HCP, compared to the $28-57 \%$ reported by other studies, had also contacted all participants by telephone within 10 days of receiving mailed results ${ }^{31}$. During this call results were explained further and participants had the chance to ask questions. This approach may have prevented the need to contact a HCP, and may 
offer a suggestion for post-test communication. Other examples of how disease risk information may be communicated effectively is given by Lautenbach et al. ${ }^{39}$.

Many consumers shared with family and/or friends. Particularly sharing with family, due to shared genetics, may also lead to improved lifestyle among individuals who did not undergo testing. However, as effects on the lifestyle of test participants currently prove to be modest, the effect on the non-tested individual is likely to be minimal.

\section{Different values of DTC-GT}

Although it is tempting to base regulatory decisions regarding DTC-GT on its potential to improve health (i.e. preventive value or clinical utility), health should not be the only criterion to judge by. A different but related value of DTC-GT is one of personal value and autonomy ${ }^{2,40}$. Several studies on reasons of purchasing DTC-GT revealed that the main reason is satisfying curiosity of the consumer ${ }^{41-43}$, indicating that the entertainment value should receive considerable attention in justifying these test $\mathrm{s}^{40}$. In addition, it may serve as a source of information for informed health-related decision-making, such as long-term life decisions. The value of genetic testing for individuals who purchase it with the purpose of learning of disease risks to make informed health decisions, may be of a very different nature from the value for those purchasing out of mere interest and entertainment. Therefore, self-selection of undergoing testing for health-related reasons may be an important determinant of finding an effect, as a recent meta-analysis ${ }^{6}$ found no effect of receiving genetic information on behaviour change in randomised trials. To use DTC-GT as an effective health behaviour intervention, it may need to be combined with continued lifestyle intervention and/or counselling, which will need tailoring to the intentions of the individual. Doing so, will principally attract more consumers with the intention of improving health, but will also stimulate those with and without prior intention of improving health in the process of behaviour change. The effects on health behaviour may then be much larger compared to the effects found from genetic testing like most DTC-GT companies currently offer, which may more often be purchased out of mere entertainment.

\section{Demographics of the study populations}

A common criticism is the assumption that most DTC-GT users involve predominantly white individuals with higher income and education level. Kaufman et $\mathrm{al}^{10}$ explicitly compared their study population with the general US population and found that white men with higher incomes and education were indeed overrepresented in their study. Although other studies did no direct comparisons to the general population, most studies support this finding. If DTC-GT services indeed stimulate healthier lifestyles, this finding could further contribute to socio-economic health differences. 


\section{Limitations}

The current study carries several limitations, which is partly due to limitations in the primary studies. Firstly, the reported estimates are mostly not compared to a control group and may reflect natural behaviour changes. However, as several studies specifically included "as a result of receiving your report" in their questions, the effect might be mitigated. Secondly, self-reporting of behaviour change and social desirability might have lead to reporting bias and an overestimation of the effect. Therefore, future studies should include more objective forms of behaviour measurements. Thirdly, estimates of the percentage of people with any positive lifestyle change as well as those who shared with any HCP may have been underestimated. This is due to studies reporting percentages for each behaviour or HCP separately, which could not simply be combined into one summary estimate as participants may changed multiple behaviours or shared with multiple HCPs resulting in participants being counted twice. To reduce the effect as much as possible, only the most overall estimate was included. Fourthly, the five publications of the Scripps Genomic Health Initiative ${ }^{5,25, ~ 26, ~ 28, ~} 29$ were considered nonactual consumers due to the recruitment through the research project. However, it should be noted that the participants were exposed to the same Navigenics advertising, imagery, and information as regular Navigenics customers, in addition to the studyrelated information. Fifthly, some meta-analyses are based on a small number of studies, and these estimates should therefore be interpreted with caution. Finally, high levels of heterogeneity were found in the meta-analyses, remaining after sub-analyses by actual consumers vs. non-actual consumers. Possible explanations may include the specific behaviour changes that were studied and combined (e.g. "Healthier diet" vs. "More careful about diet"), differences in post-test contact and follow-up duration of the included studies, and different study designs used. Unfortunately, no subgroup analyses were possible on duration of follow-up due to the wide range of follow-up durations within and between studies. Longer duration of follow-up is likely to reduce the percentage of people who have maintained a changed lifestyle and increase the percentage of people who have shared their results ${ }^{5,25}$.

\section{Conclusion}

Although DTC-GT has the potential to be cost-effective as a health intervention, both the genetic testing and subsequent actions (such as post-test counselling or additional lifestyle interventions) will have to be offered in the right way to the right target audience with tailored follow-up. In order to identify and target this population, research is needed on the characteristics of consumers who do and do not change behaviour or experience adverse psychological responses, and who need or desire additional medical attention. Only then can DTC-GT be used, other than for its entertainment value, for the population at whole with maximum benefits at minimum costs. 


\section{Funding}

This project was funded by the Maastricht University Interfaculty Program 'Eatwell'. The funder had no influence in the design or execution of the research.

\section{Conflict of interest}

Kelly Stewart, Anke Wesselius, Maartje Schreurs and Annemie Schols declare they have no conflict of interest. A spin off genetic prevention service that aims to provide information to health professionals may be launched in the future, which will then be led by Maurice Zeegers.

\section{Ethical approval}

This article does not contain any studies with human participants or animals performed by any of the authors. 
Chapter 4 | Behavioural changes, sharing behaviour and psychological responses after DTC-GT

\section{References}

1. Rafiq M, Ianuale C, Ricciardi W and Boccia S. Direct-to-consumer genetic testing: A systematic review of european guidelines, recommendations, and position statements. Genetic testing and molecular biomarkers. 2015; 19: 535-47.

2. Vayena E. Direct-to-consumer genomics on the scales of autonomy. Journal of medical ethics. 2015; 41: 310-4.

3. Bloss CS, Madlensky L, Schork NJ and Topol EJ. Genomic information as a behavioral health intervention: Can it work? Personalized Medicine. 2011; 8: 659-67.

4. Vernarelli JA, Roberts JS, Hiraki S, Chen CA, Cupples LA and Green RC. Effect of Alzheimer disease genetic risk disclosure on dietary supplement use. The American journal of clinical nutrition. 2010; 91: 1402-7.

5. Bloss CS, Wineinger NE, Darst BF, Schork NJ and Topol EJ. Impact of direct-to-consumer genomic testing at long term follow-up. Journal of medical genetics. 2013; 50: 393-400.

6. Hollands GJ, French DP, Griffin SJ, et al. The impact of communicating genetic risks of disease on risk-reducing health behaviour: systematic review with meta-analysis. BMJ (Clinical research ed). 2016; 352: i1102.

7. Smerecnik C, Grispen JE and Quaak M. Effectiveness of testing for genetic susceptibility to smoking-related diseases on smoking cessation outcomes: a systematic review and meta-analysis. Tobacco control. 2012; 21: 347-54.

8. Covolo L, Rubinelli S, Ceretti E and Gelatti U. Internet-Based Direct-to-Consumer Genetic Testing: A Systematic Review. Journal of medical Internet research. 2015; 17.

9. Egglestone C, Morris A and O'Brien A. Effect of direct-to-consumer genetic tests on health behaviour and anxiety: a survey of consumers and potential consumers. Journal of genetic counseling. 2013; 22: 565-75.

10. Kaufman DJ, Bollinger JM, Dvoskin RL and Scott JA. Risky business: risk perception and the use of medical services among customers of DTC personal genetic testing. Journal of genetic counseling. 2012; 21: 413-22.

11. Lippi G, Favaloro E and Plebani M. Direct-to-consumer testing: more risks than opportunities. International journal of clinical practice. 2011; 65: 1221-9.

12. Nordgren A. Neither as harmful as feared by critics nor as empowering as promised by providers: risk information offered direct to consumer by personal genomics companies. Journal of community genetics. 2014; 5: 59-68.

13. McGuire AL and Burke W. An unwelcome side effect of direct-to-consumer personal genome testing: raiding the medical commons. Jama. 2008; 300: 2669-71.

14. Fausset CB. Comprehension of health risk probabilities: the roles of age, numeracy, format, and mental representation. 2012.

15. McBride CM, Wade $\mathrm{CH}$ and Kaphingst KA. Consumers' views of direct-to-consumer genetic information. Annual review of genomics and human genetics. 2010; 11: 427-46.

16. Freeman MF and Tukey JW. Transformations related to the angular and the square root. The Annals of Mathematical Statistics. 1950: 607-11.

17. Nyaga VN, Arbyn M and Aerts M. Metaprop: a Stata command to perform meta-analysis of binomial data. Archives of public health = Archives belges de sante publique. 2014; 72: 39 .

18. Higgins J, Thompson SG, Deeks JJ and Altman DG. Measuring inconsistency in meta-analyses [journal article as teaching resource, deposited by John Flynn]. British medical journal. 2003; 327: 557-60.

19. Begg CB and Mazumdar M. Operating characteristics of a rank correlation test for publication bias. Biometrics. 1994: 1088-101.

20. Egger M, Smith GD, Schneider M and Minder C. Bias in meta-analysis detected by a simple, graphical test. BMJ (Clinical research ed). 1997; 315: 629-34.

21. Gordon ES, Griffin G, Wawak L, Pang H, Gollust SE and Bernhardt BA. "It's Not Like Judgment 
Chapter 4 | Behavioural changes, sharing behaviour and psychological responses after DTC-GT

Day": Public Understanding of and Reactions to Personalized Genomic Risk Information. Journal of genetic counseling. 2012; 21: 423-32.

22. Lee SSJ, Vernez SL, Ormond KE and Granovetter M. Attitudes towards social networking and sharing behaviors among consumers of direct-to-consumer personal genomics. Journal of Personalized Medicine. 2013; 3: 275-87.

23. McGrath SP, Coleman J, Najjar L, Fruhling A and Bastola DR. Comprehension and Data-Sharing Behavior of Direct-To-Consumer Genetic Test Customers. Public health genomics. 2016; 19: 11624.

24. McGuire AL, Diaz CM, Wang T and Hilsenbeck SG. Social networkers' attitudes toward direct-toconsumer personal genome testing. The American journal of bioethics : AJOB. 2009; 9: 3-10.

25. Bloss CS, Schork NJ and Topol EJ. Effect of direct-to-consumer genomewide profiling to assess disease risk. The New England journal of medicine. 2011; 364: 524-34.

26. Boeldt DL, Schork NJ, Topol EJ and Bloss CS. Influence of individual differences in disease perception on consumer response to direct-to-consumer genomic testing. Clinical genetics. 2015; 87: 225-32.

27. Carere DA, Kraft P, Kaphingst KA, Roberts JS and Green RC. Consumers report lower confidence in their genetics knowledge following direct-to-consumer personal genomic testing. Genetics in medicine: official journal of the American College of Medical Genetics. 2016; 18: 65.

28. Darst BF, Madlensky L, Schork NJ, Topol EJ and Bloss CS. Perceptions of genetic counseling services in direct-to-consumer personal genomic testing. Clinical genetics. 2013; 84: 335-9.

29. Darst BF, Madlensky L, Schork NJ, Topol EJ and Bloss CS. Characteristics of genomic test consumers who spontaneously share results with their health care provider. Health communication. 2014; 29: 105-8.

30. van der Wouden CH, Carere DA, Maitland-van der Zee AH, Ruffin MT, Roberts JS and Green RC. Consumer perceptions of interactions with primary care providers after direct-to-consumer personal genomic testing. Annals of internal medicine. 2016.

31. Kaphingst KA, McBride CM, Wade C, et al. Patients' understanding of and responses to multiplex genetic susceptibility test results. Genetics in medicine : official journal of the American College of Medical Genetics. 2012; 14: 681-7.

32. Reid RJ, McBride CM, Alford SH, et al. Association between health-service use and multiplex genetic testing. Genetics in medicine : official journal of the American College of Medical Genetics. 2012; 14: 852-9.

33. O’Neill SC, Tercyak KP, Baytop C, Hensley Alford S and McBride CM. A New Approach to Assessing Affect and the Emotional Implications of Personal Genomic Testing for Common Disease Risk. Public health genomics. 2015; 18: 104-12.

34. Haga SB, Barry WT, Mills R, et al. Impact of Delivery Models on Understanding Genomic Risk for Type 2 Diabetes. Public Health Genomics. 2014; 17: 95-104.

35. James KM, Cowl CT, Tilburt JC, et al. Impact of direct-to-consumer predictive genomic testing on risk perception and worry among patients receiving routine care in a preventive health clinic. Mayo Clinic proceedings. 2011; 86: 933-40.

36. Olfson E, Hartz S, Carere DA, et al. Implications of personal genomic testing for health behaviors: the case of smoking. Nicotine \& Tobacco Research. 2016: ntw168.

37. Myung S-K, Kim Y, Ju W, Choi H and Bae W. Effects of antioxidant supplements on cancer prevention: meta-analysis of randomized controlled trials. Annals of Oncology. 2010; 21: 166-79.

38. Giovanni MA, Fickie MR, Lehmann LS, et al. Health-care referrals from direct-to-consumer genetic testing. Genetic Testing and Molecular Biomarkers. 2010; 14: 817-9.

39. Lautenbach DM, Christensen KD, Sparks JA and Green RC. Communicating genetic risk information for common disorders in the era of genomic medicine. Annual review of genomics and human genetics. 2013; 14.

40. Chung MWH and Ng JCF. Personal utility is inherent to direct-to-consumer genomic testing. Journal of medical ethics. 2016: medethics-2015-103057. 
Chapter 4 | Behavioural changes, sharing behaviour and psychological responses after DTC-GT

41. Gollust SE, Gordon ES, Zayac C, et al. Motivations and perceptions of early adopters of personalized genomics: perspectives from research participants. Public health genomics. 2012; 15: 22-30.

42. Ormond KE, Hudgins L, Ladd JM, Magnus DM, Greely HT and Cho MK. Medical and graduate students' attitudes toward personal genomics. Genetics in Medicine. 2011; 13: 400-8.

43. Su Y, Howard HC and Borry P. Users' motivations to purchase direct-to-consumer genome-wide testing: an exploratory study of personal stories. Journal of community genetics. 2011; 2: 135-46. 
Online resource 1: Forest plots of all meta-analyses

\section{Any positive lifestyle change}

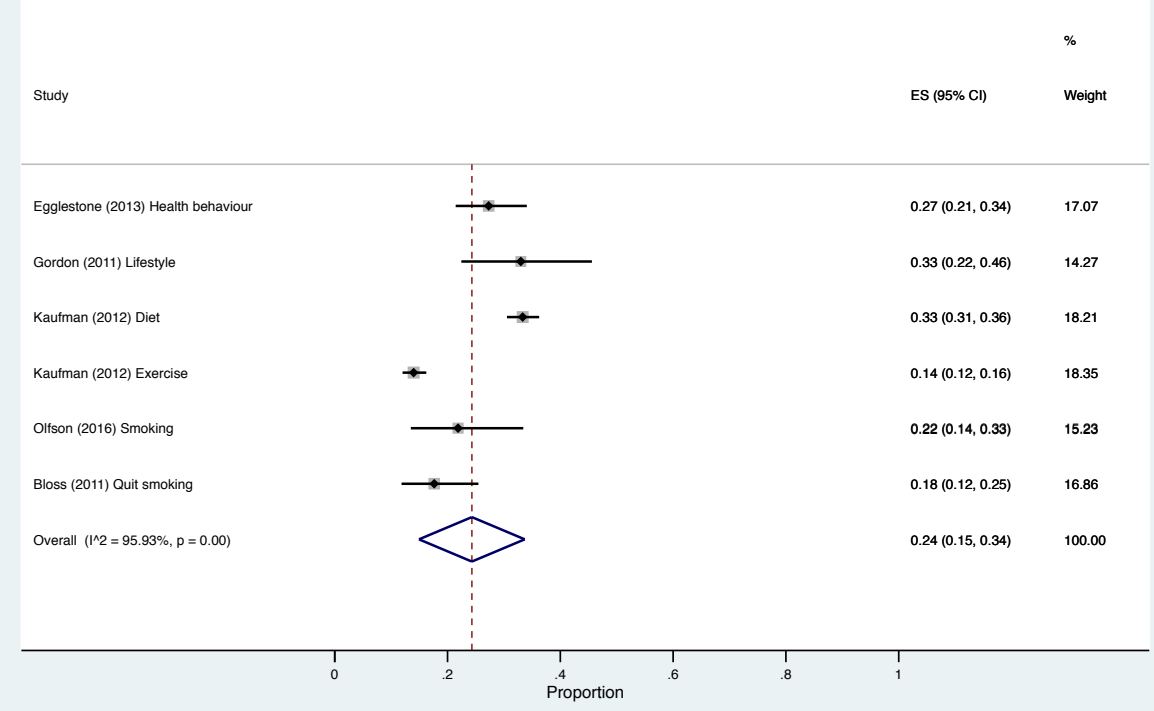

Figure 1 Overall meta-analysis of any positive lifestyle change, expressed in proportion of participants changed 


\section{Any positive lifestyle change \\ by customer type}

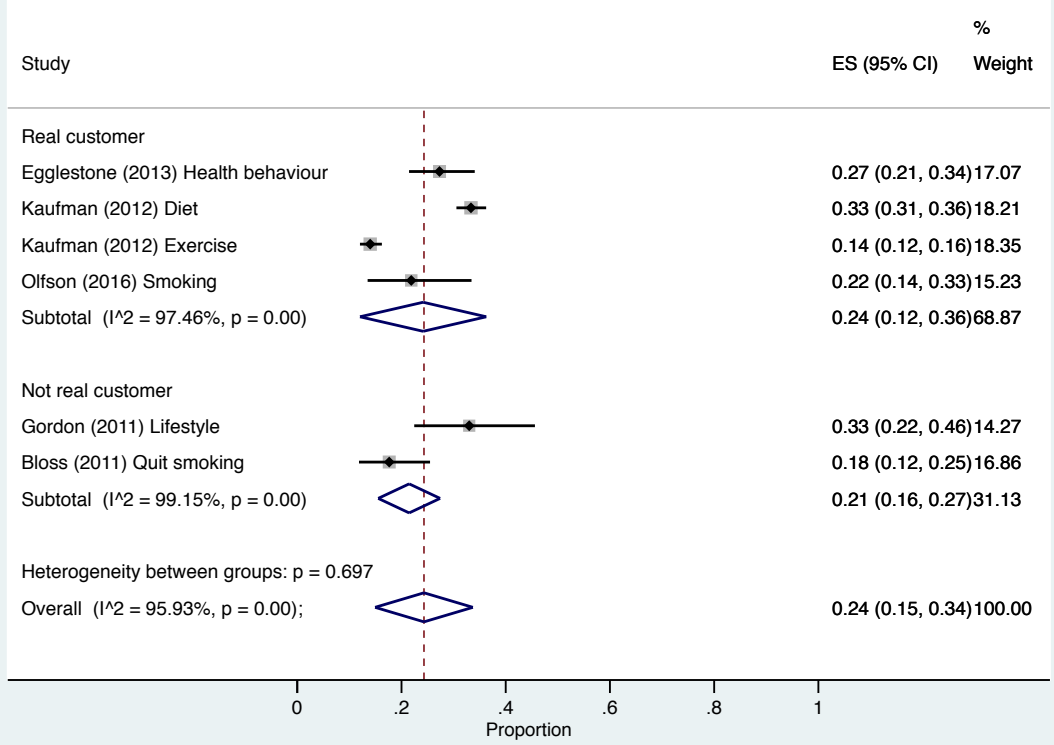

Figure 2 Meta-analysis of any positive lifestyle change by consumer type, expressed in proportion of participants changed

\section{Any positive lifestyle change \\ by price paid}

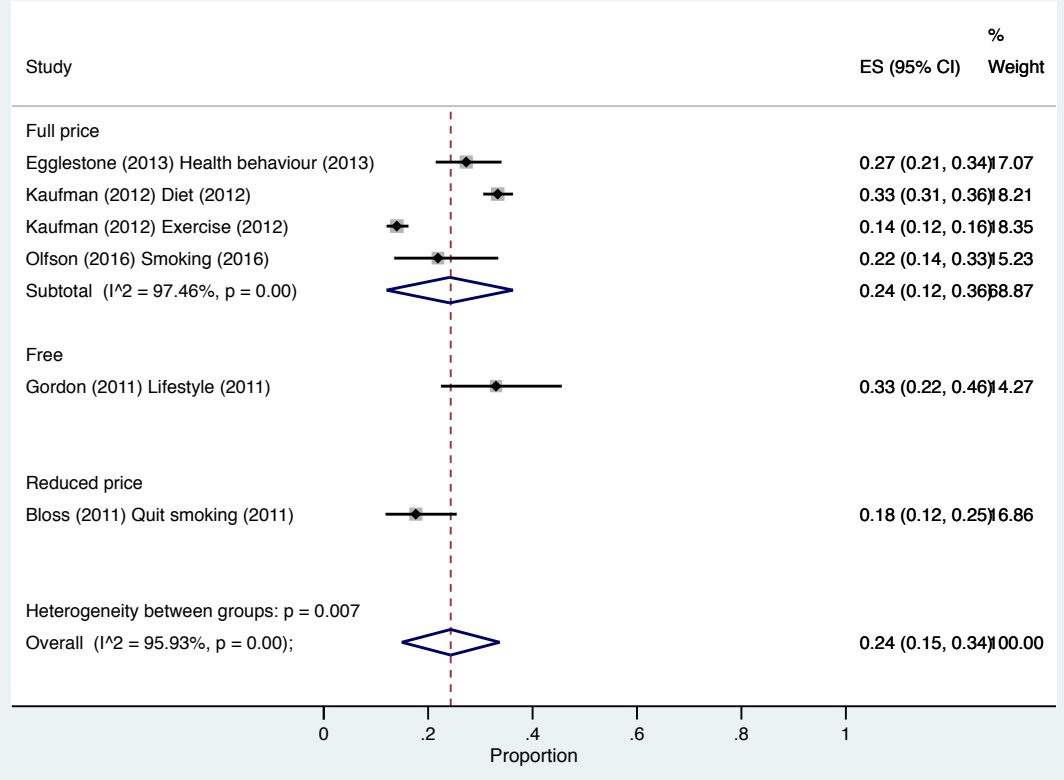

Figure 3 Meta-analysis of any positive lifestyle change by price paid, expressed in proportion of participants changed 


\section{Improved dietary practices}

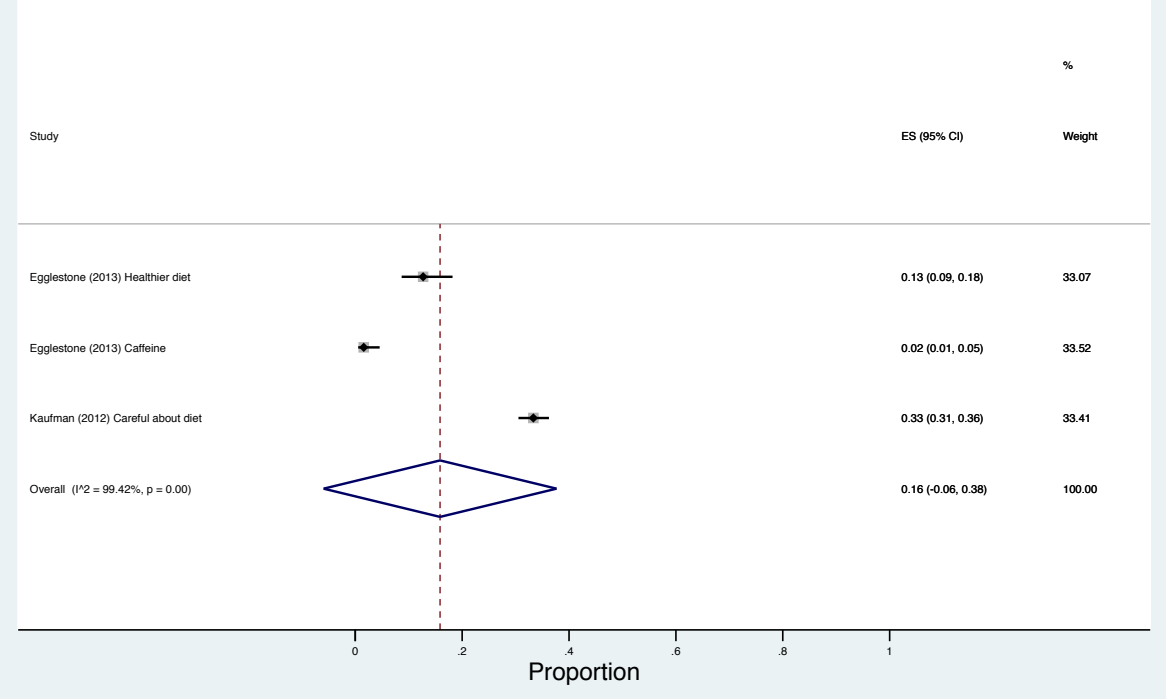

Figure 4 Overall meta-analysis of improved dietary practices, expressed in proportion of participants changed

\section{Improved exercise practices}

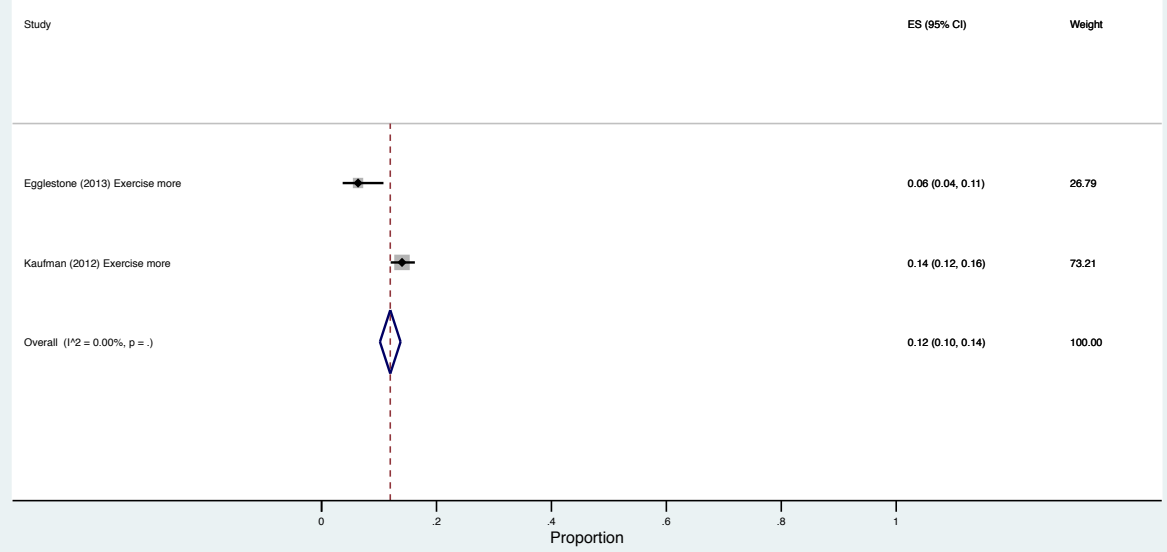

Figure 5 Overall meta-analysis of improved exercise practices, expressed in proportion of participants changed 


\section{Quit smoking}

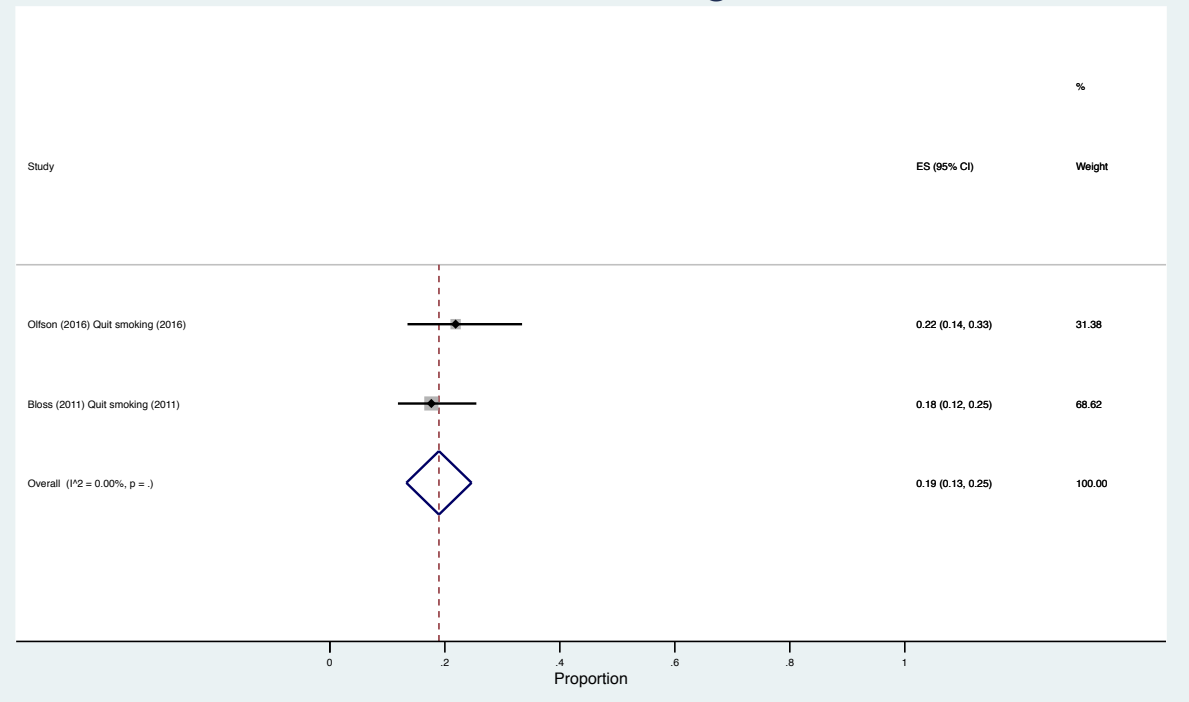

Figure 6 Overall meta-analysis of quitting smoking, expressed in proportion of pre-test smokers who had quit

\section{Change in supplement use}

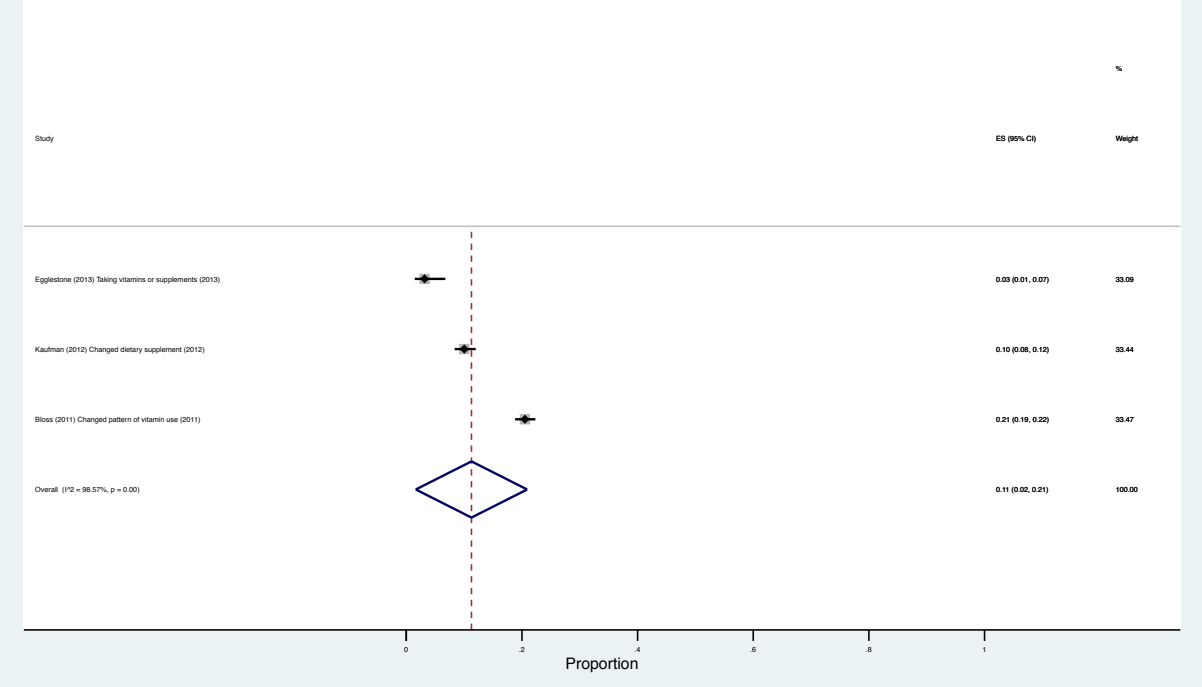

Figure 7 Overall meta-analysis of change in supplement use, expressed in proportion of participants changed 


\section{Change in supplement use \\ by customer type}

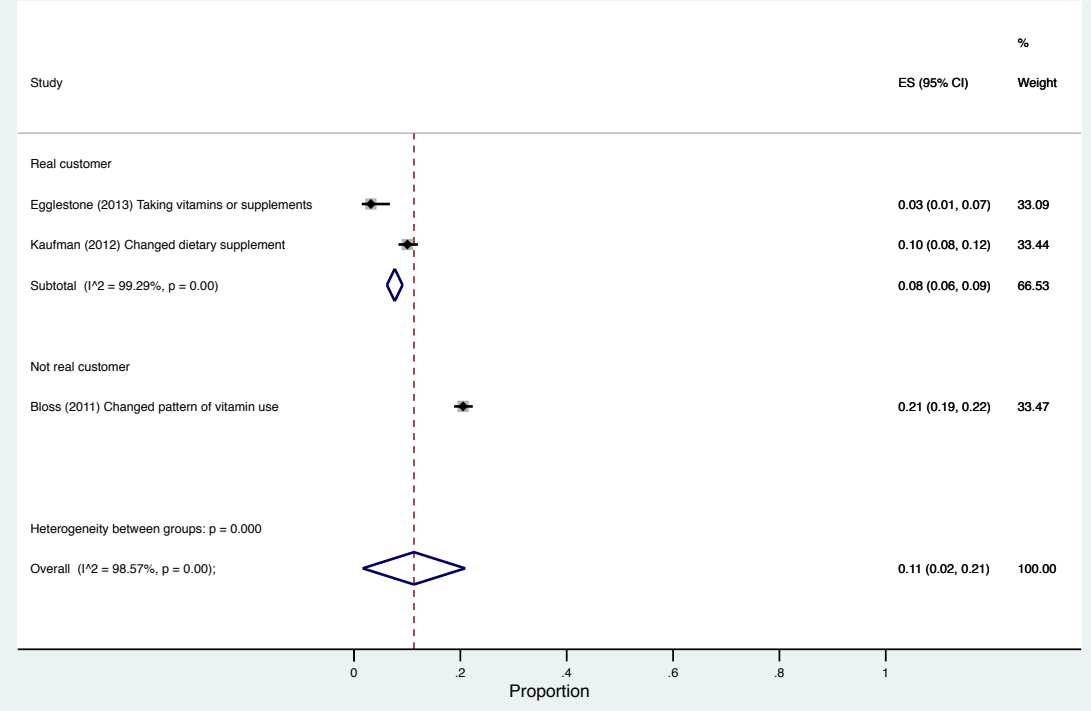

Figure 8 Meta-analysis of change in supplement use by consumer type, expressed in proportion of participants changed

\section{Change in supplement use \\ by price paid}

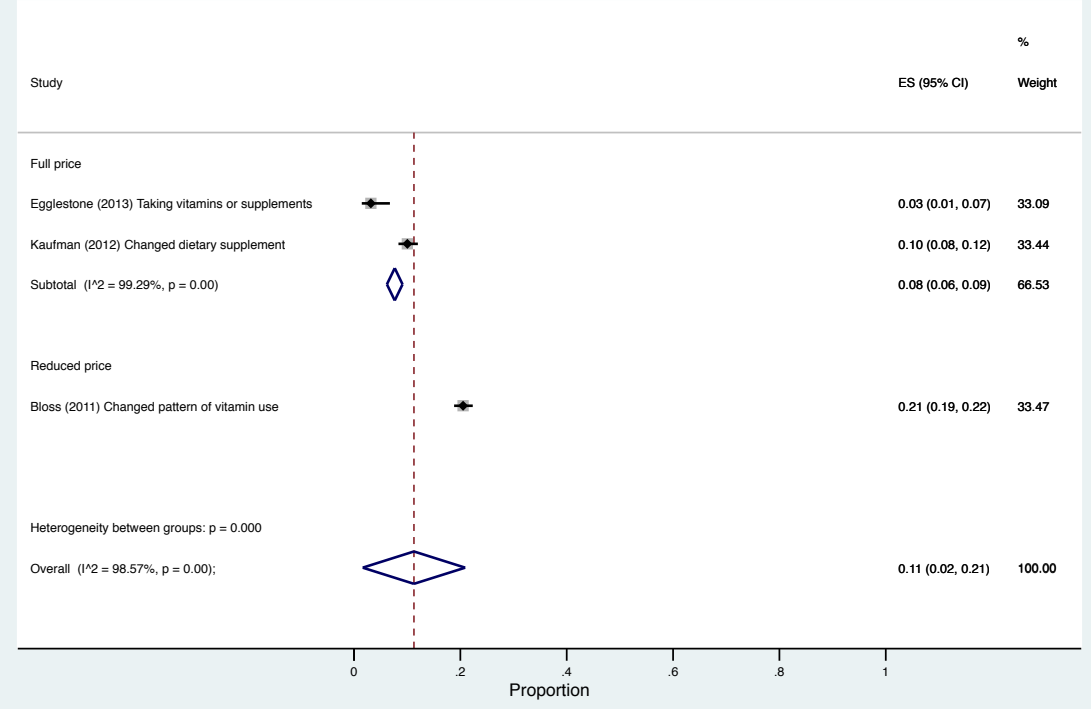

Figure 9 Meta-analysis of change in supplement use by price paid, expressed in proportion of participants changed 


\section{Information-seeking behaviour}

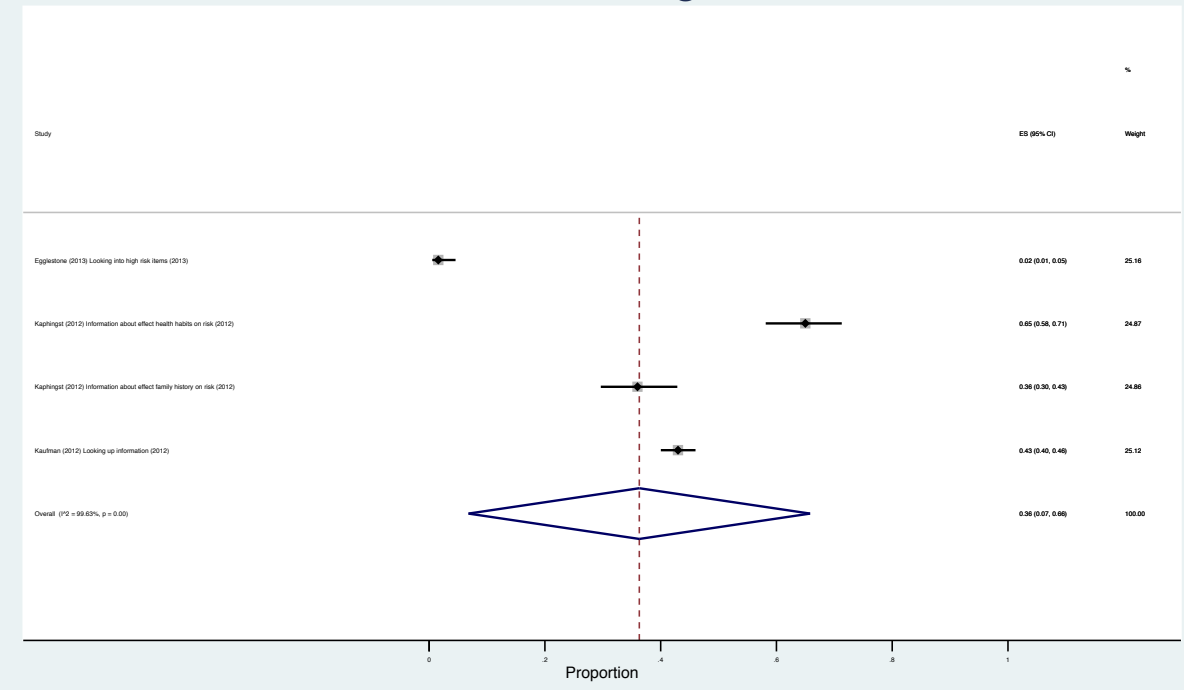

Figure 10 Overall meta-analysis of information-seeking behaviour, expressed in proportion of participants who sought information

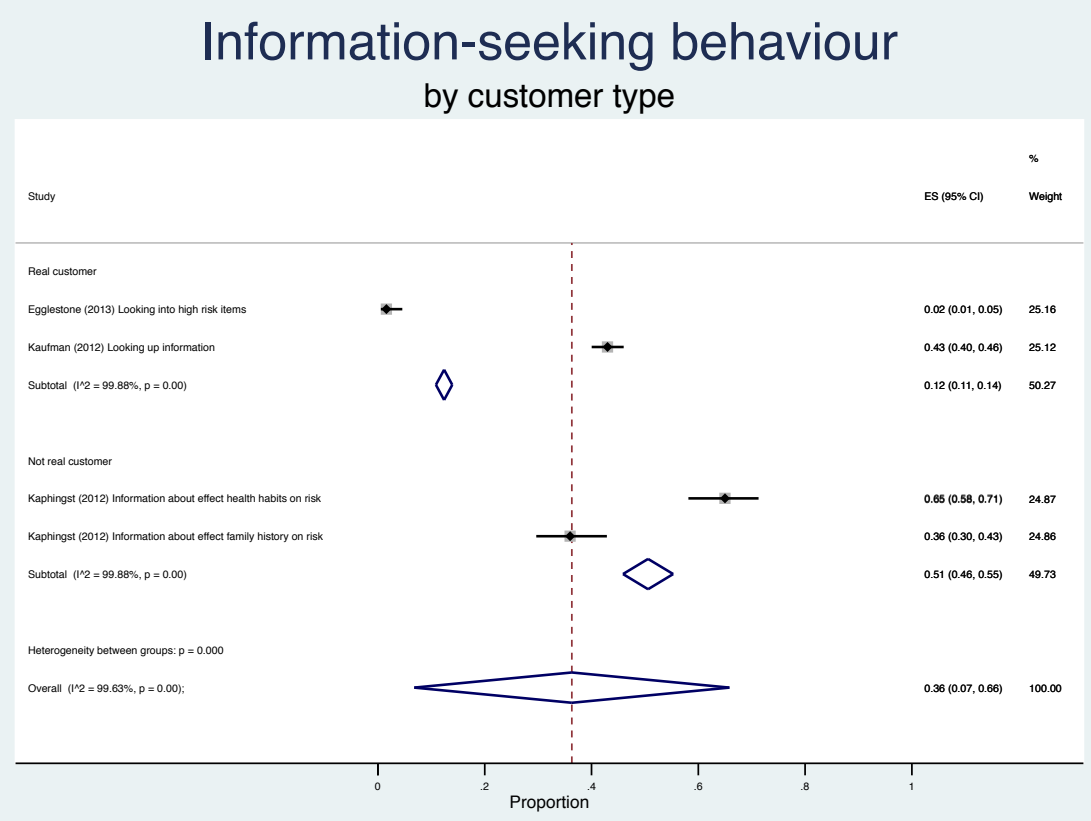

Figure 11 Meta-analysis of information-seeking behaviour by customer type, expressed in proportion of participants who sought information 


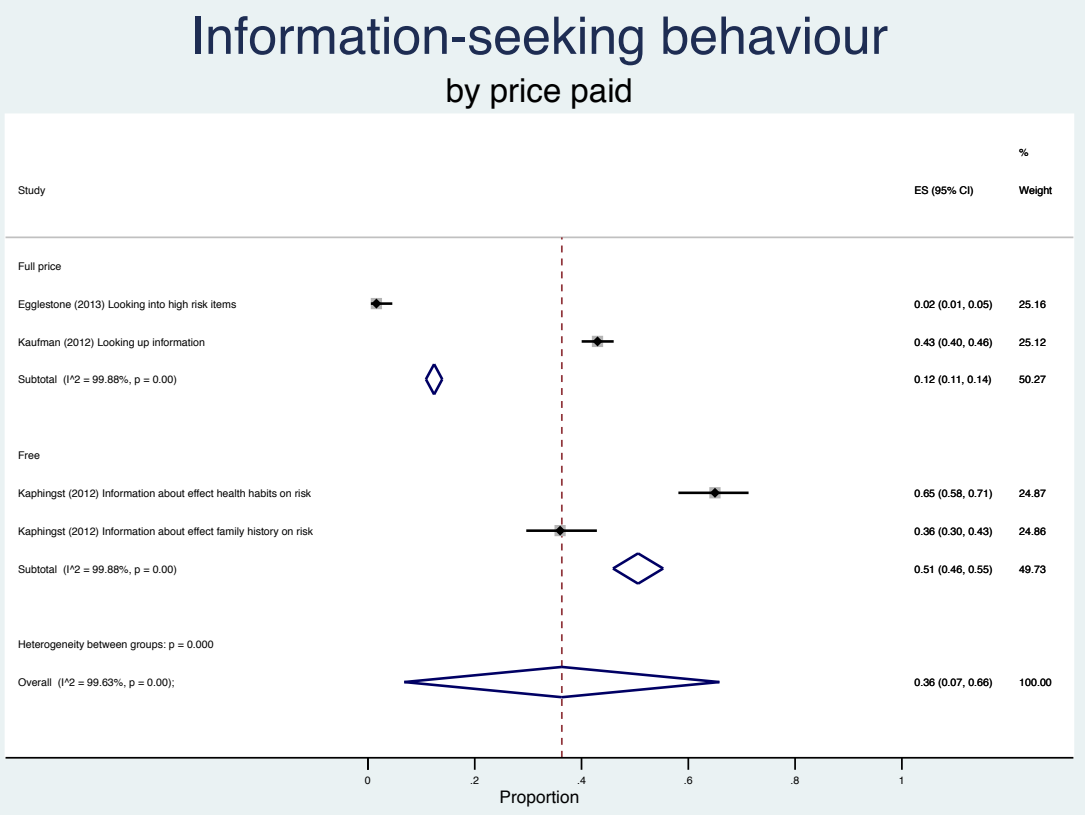

Figure 12 Meta-analysis of information-seeking behaviour by price paid, expressed in proportion of participants who sought information

Sharing with (at least one) health care professional

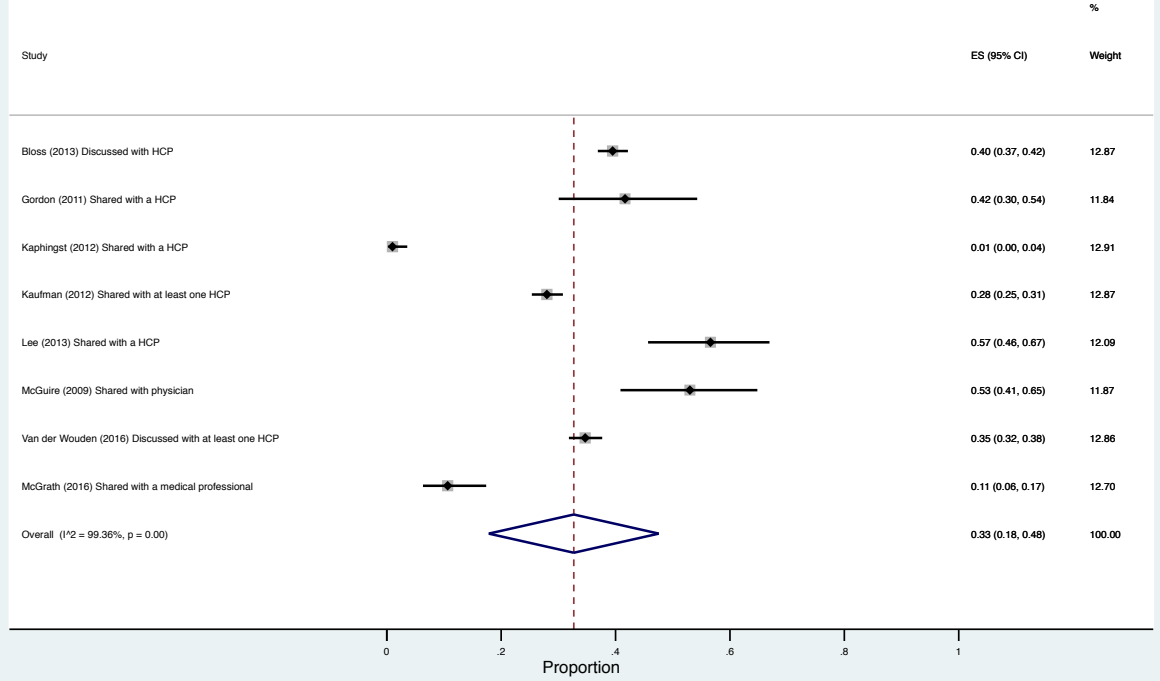

Figure 13 Overall meta-analysis of sharing with (at least one) health care professional, expressed in proportion of participants who shared 


\section{Sharing with (at least one) health care professional} by customer type

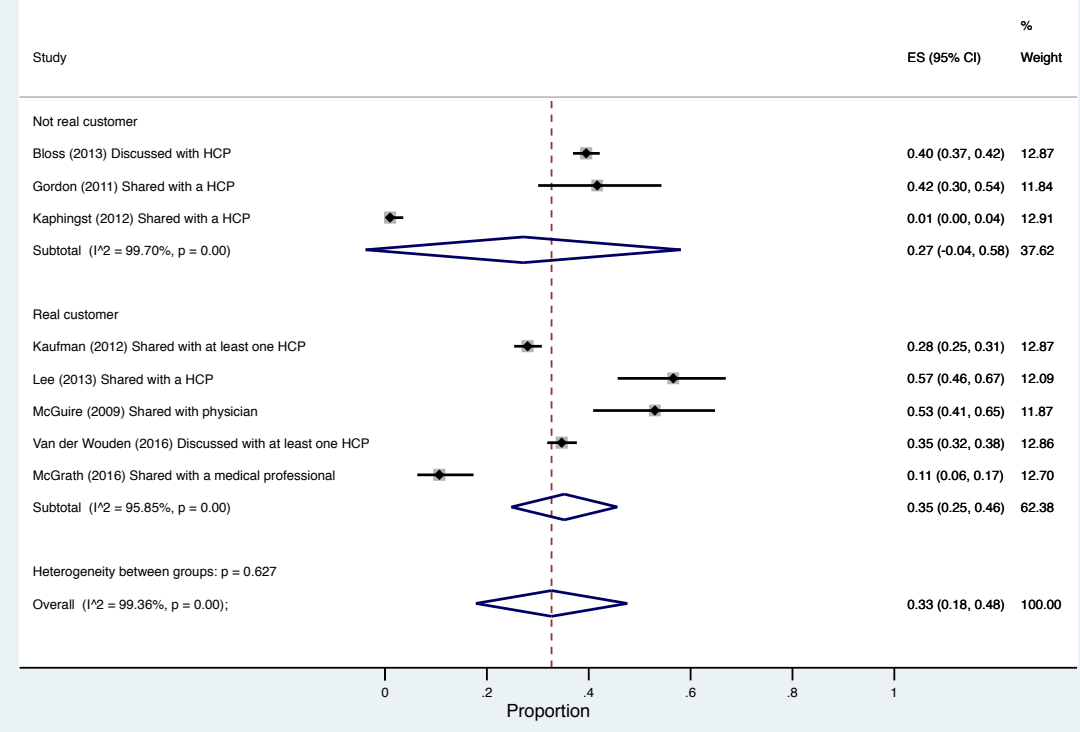

Figure 14 Meta-analysis of sharing with (at least one) health care professional by consumer type, expressed in proportion of participants who shared

\section{Sharing with (at least one) health care professional}

\section{by price paid}

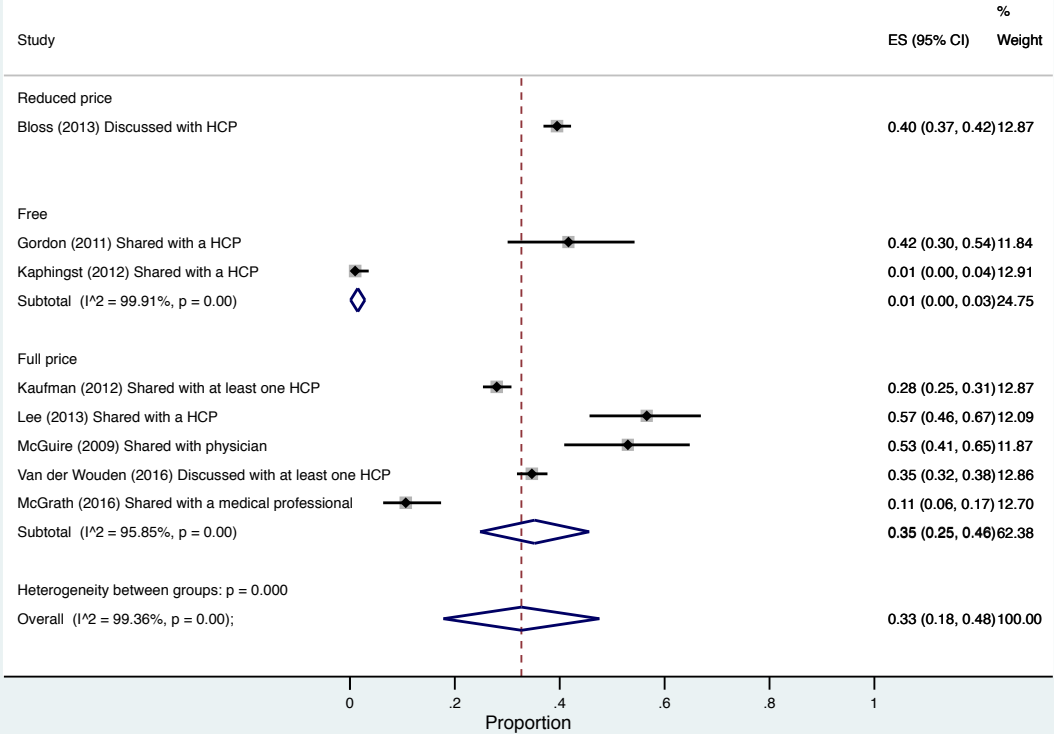

Figure 15 Meta-analysis of sharing with (at least one) health care professional by price paid, expressed in proportion of participants who shared 


\section{Sharing with general practitioner}

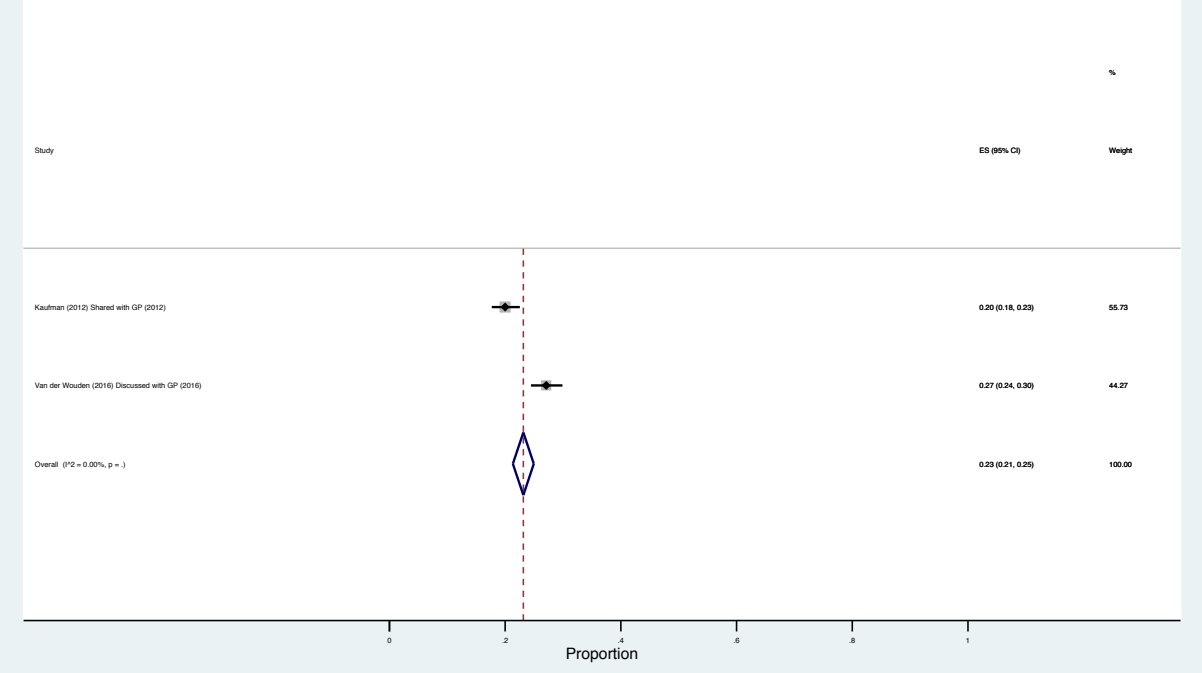

Figure 16 Overall meta-analysis of sharing with a general practitioner, expressed in proportion of participants who shared

\section{Sharing with genetic specialist}

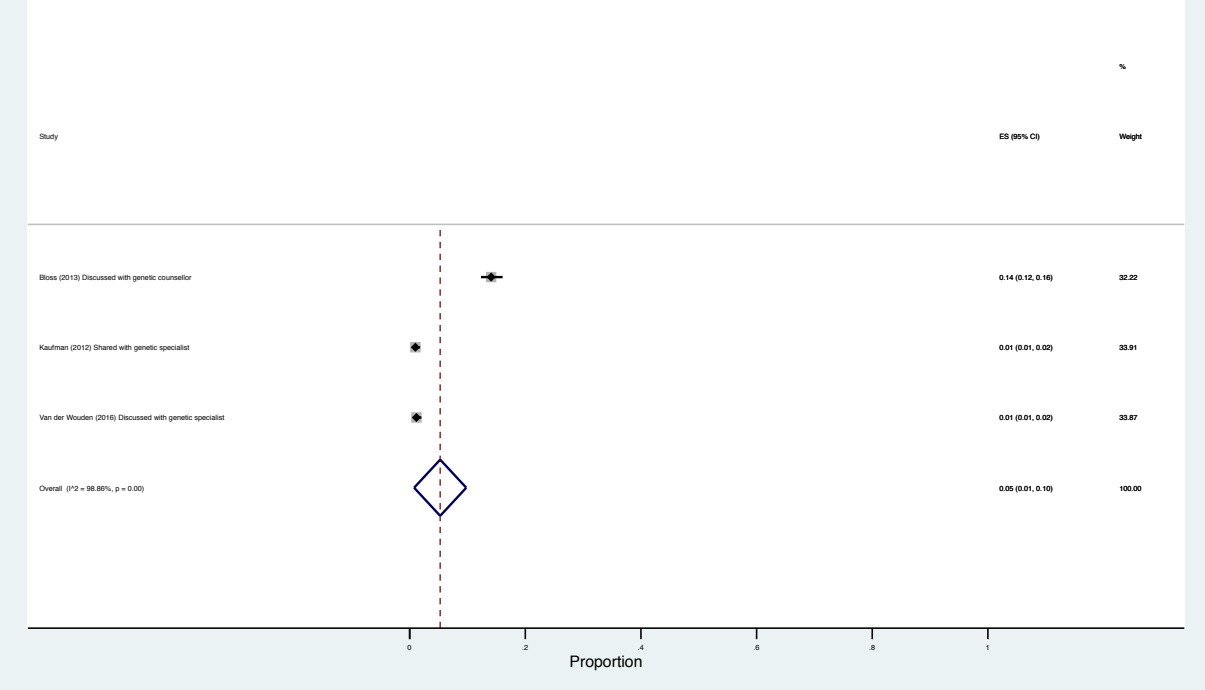

Figure 17 Overall meta-analysis of sharing with a genetic specialist, expressed in proportion of participants who shared 


\section{Sharing with genetic specialist \\ by customer type}

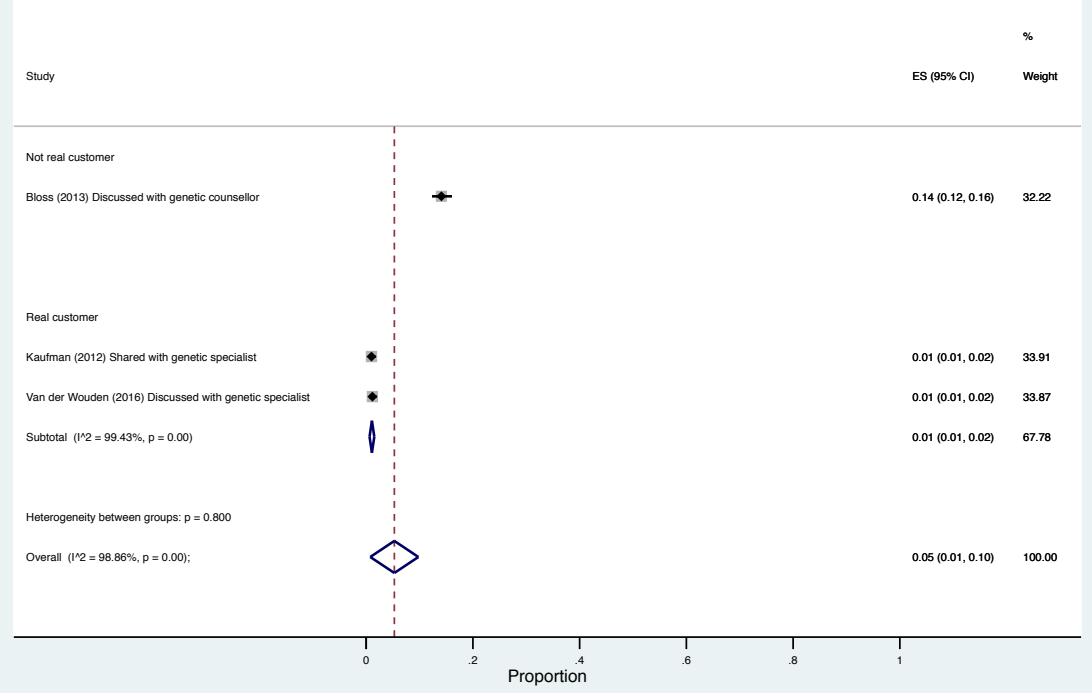

Figure 18 Meta-analysis of sharing with a genetic specialist by consumer type, expressed in proportion of participants who shared

\section{Sharing with genetic specialist \\ by price paid}

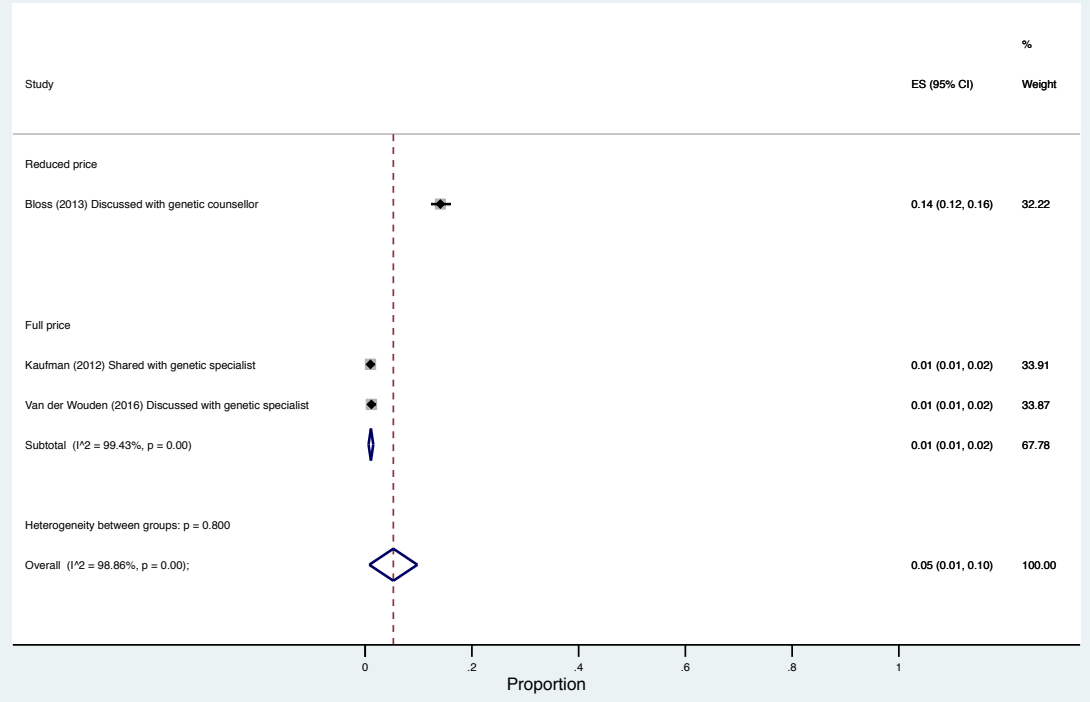

Figure 19 Meta-analysis of sharing with a genetic specialist by price paid, expressed in proportion of participants who shared 


\section{Sharing with family and/or friends}

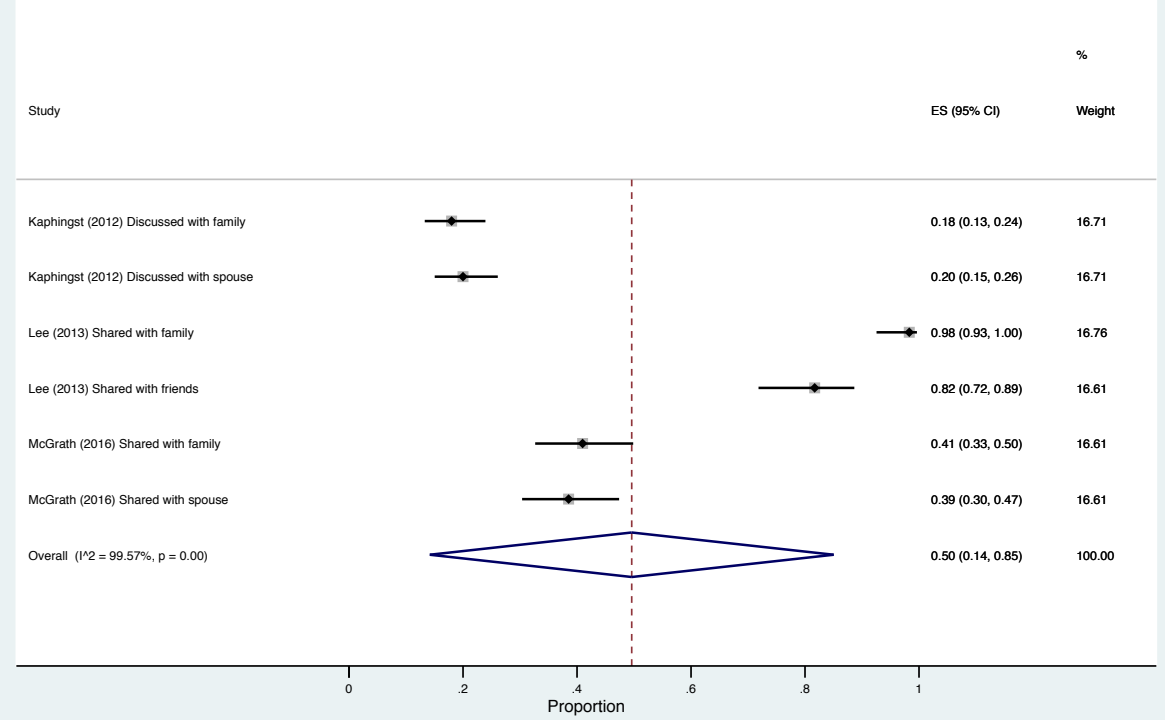

Figure 20 Overall meta-analysis of sharing with family and/or friends, expressed in proportion of participants who shared

\section{Sharing with family and/or friends \\ by customer type}

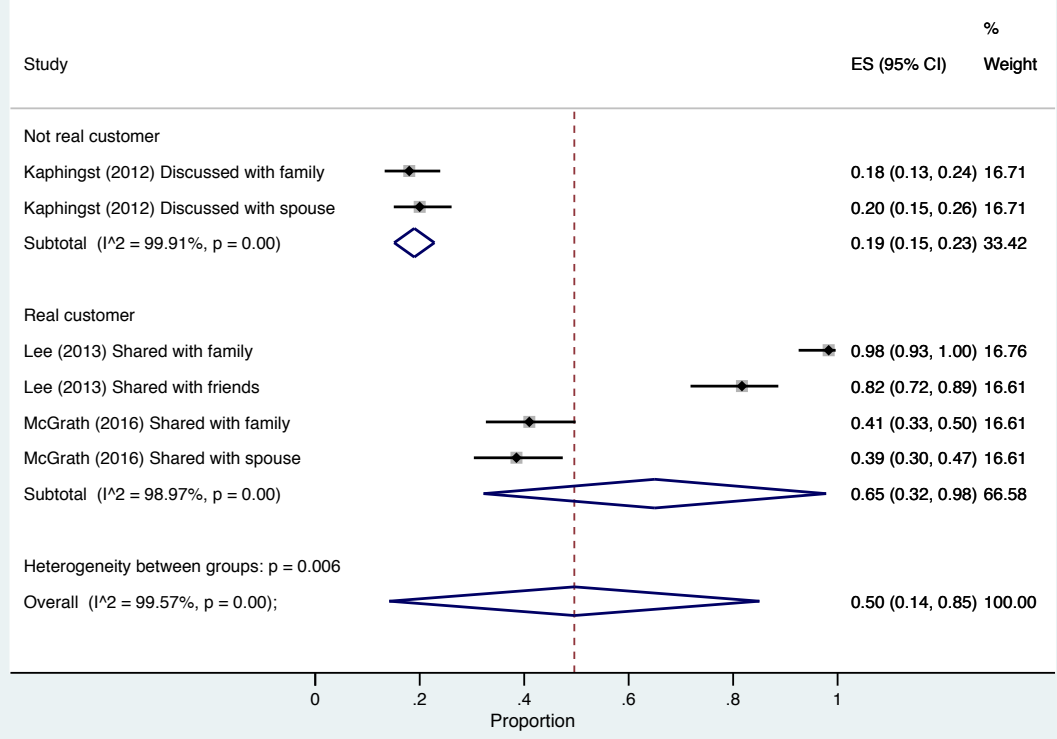

Figure 21 Meta-analysis of sharing with family and/or friends by customer type, expressed in proportion of participants who shared 


\section{Sharing with family and/or friends}

by price paid

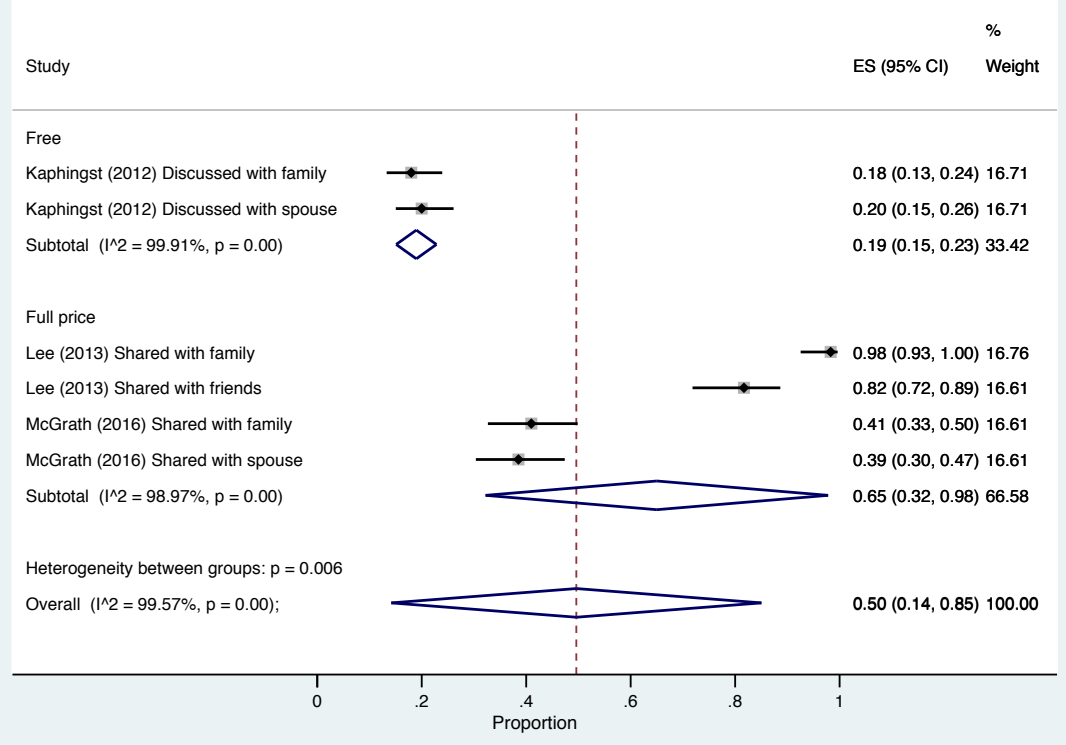

Figure 22 Meta-analysis of sharing with family and/or friends by price paid, expressed in proportion of participants who shared

\section{Preventive checks}

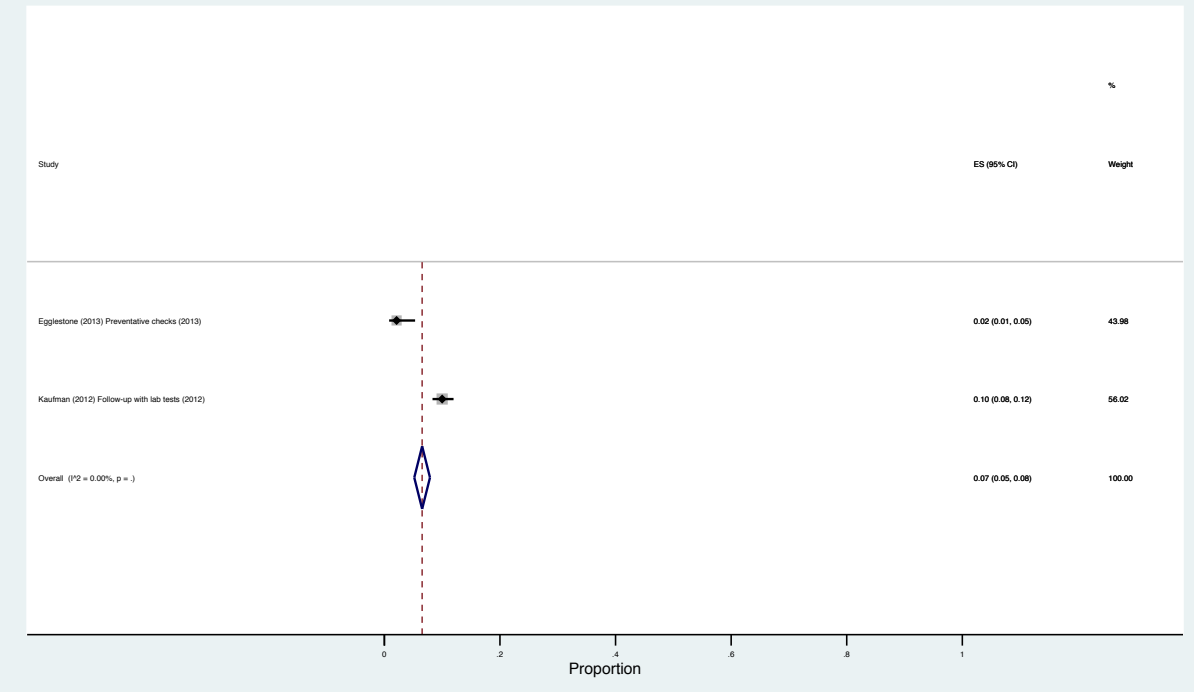

Figure 23 Overall meta-analysis of attending preventive checks, expressed in proportion of participants who had preventive checks 


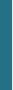




\section{Abstract}

Each year, 430000 people are diagnosed with bladder cancer. Due to the high recurrence rate of the disease, primary prevention is paramount. Therefore, we reviewed all meta-analyses on modifiable risk factors of primary bladder cancer. PubMed, Embase and Cochrane database were systematically searched for metaanalyses on modifiable risk factors published between 1995 and 2015. When appropriate, meta-analyses (MA) were combined in meta-meta-analysis (MMA). If not, the most comprehensive MA was selected based on the number of primary studies included. Probability of causation was calculated for individual factors and a subset of lifestyle factors combined. Of 1,496 articles identified, 5 were combined in MMA and 21 were most comprehensive on a single risk factor. Statistically significant associations were found for current (RR 3.14) or former (RR 1.83) cigarette smoking, pipe (RR 1.9) or cigar (RR 2.3) smoking, antioxidant supplementation (RR 1.52), obesity (RR 1.10), higher physical activity levels (RR 0.86), higher body levels of selenium (RR 0.61) and vitamin D (RR 0.75), and higher intakes of: processed meat (RR 1.22), vitamin A (RR 0.82), vitamin $E$ ( $R R$ 0.82 ), folate (RR 0.84), fruit (RR 0.77), vegetables ( $R R$ 0.83), citrus fruit (RR 0.85 ), and cruciferous vegetables (RR 0.84). Finally, three occupations with the highest risk were tobacco workers (RR 1.72), dye workers (RR 1.58), and chimney sweeps (RR 1.53). The probability of causation for individual factors ranged from $4 \%-68 \%$. The combined probability of causation was $81.8 \%$. Modification of lifestyle and occupational exposures can considerably reduce the bladder cancer burden. While smoking remains one of the key risk factors, also several diet-related and occupational factors are very relevant.

Review registration: PROSPERO CRD42015023411

Keywords: Bladder cancer, Prevention, Risk factors, Lifestyle, Obesity, Occupation, Smoking 


\section{Introduction}

The International Agency for Research on Cancer (IARC) estimated that worldwide 430,000 people were diagnosed with bladder cancer in 2012, with an age-standardised rate (ASR) of 5.3 per 100,000 people. Incidence of bladder cancer is particularly high in males with $77 \%$ of the cases, ranking it the 7 th highest on incidence and 9 th highest on mortality. For females it is the 19th highest on incidence and 17th highest on mortality. The incidence of bladder cancer is almost 3 times higher in more developed countries compared to less developed countries (ASR of 9.5 and 3.3 per 100,000 respectively). ${ }^{1}$

Several genetic polymorphisms have been proposed to be associated with the development of bladder cancer. However, genetic effects can explain 'only' $7 \%$ of the bladder cancer incidence in western populations, ${ }^{2}$ and therefore, risk factors related to lifestyle, environmental, and occupational exposures could potentially play an important role in the development of this disease. Over the years, multiple external risk factors have been proposed. Well-established risk factors are tobacco consumption, occupational exposure, such as to aromatic amines and Polycyclic Aromatic Hydrocarbons (PAHs), and infection with Schistosoma hematobium. ${ }^{3}$ Whereas smoking is the most important risk factor in developed countries, S. hematobium infection accounts for the largest burden in African countries. ${ }^{4,5}$ Additional suggested risk factors are, amongst others, alcohol consumption, coffee intake, low fruit, and vegetable consumption, low selenium and vitamin $\mathrm{E}$ intake, pollution of drinking water, and several medical treatments.

Exposure to some of the key risk factors of bladder cancer has been successfully reduced in the population. For example, the reduction in exposure to aromatic amines seemed to be directly related to a reduction in bladder cancer incidence. ${ }^{7}$ Men are generally more exposed to occupational risk factors of bladder cancer and it is estimated that $7.1 \%$ of the bladder cancer cases in men can be attributed to occupational factors ${ }^{8}$. Also, the prevalence rates of smoking have been declining significantly over the past decades, although the absolute number of smokers has been increasing due to the growing world population. ${ }^{9}$ Because men smoke more than women, the percentage of cases attributable to smoking is higher in males than in females: $42.8 \%$ (males) versus $25.7 \%$ (females) in Europe, and $34.3 \%$ (males) versus $30.1 \%$ (females) in the United States. ${ }^{10}$ This higher prevalence of smoking among men also explains part of the discrepancy in incidence between men and women. ${ }^{11}$ Despite the reduction in exposure to some risk factors, significant exposure, and thus disease, remains. This means that the burden on the health care system will remain substantial especially when considering that at least $50 \%$ of bladder cancers recur ${ }^{12}$ and long-term monitoring of patients is required, resulting in the highest per patient lifetime costs amongst all cancers. ${ }^{13}$ For example, in the US, 
the annual cost of care of bladder cancer was estimated to be \$US4 billion. Therefore, primary prevention is paramount, particularly because many of the risk factors are modifiable and therefore preventable.

Prioritising control measures should be evidence-based. To this end, an accessible and comprehensive overview is needed of the modifiable risk factors in the development of bladder cancer, including estimates of public health impact. To our knowledge, no quantitative overview has been published addressing all modifiable risk factors of bladder cancer. Therefore, our aim was to give an overview of the current state of knowledge, at the highest level of evidence, with regard to modifiable lifestyle, environmental, and occupational risk factors for development of bladder cancer by performing a systematic review of meta-analyses (MAs), in combination with a series of meta-meta-analyses (MMAs).

\section{Methods}

This systematic review was registered with PROSPERO (identification number CRD42015023411). ${ }^{14}$ Where relevant, the publication is written in accordance to the PRISMA guidelines. ${ }^{15}$

\section{Search strategy}

A systematic literature search was performed up to September 2015 in PubMed, Embase, and the Cochrane database to identify published articles of MAs and pooled analyses describing the association between lifestyle, environmental, and occupational risk factors and the development of bladder cancer. In addition, PROSPERO was searched and authors of registered but not published reviews were contacted. Finally forward and backward referencing of relevant publications was checked for additional publications.

The database was searched using the following search terms: "urinary bladder neoplasms"[MeSH Terms], "urinary bladder neoplasms"[All Fields], "bladder cancer", combined with systematic[sb], meta-analysis[Title/Abstract], meta-analysis[Publication Type], systematic review[Text Word], pooled analysis[Text Word].

\section{Selection criteria for publications}

A first screening was done based on the title and abstract. Articles were considered for inclusion if they were MAs or pooled analyses describing the association between modifiable risk factors and development of bladder cancer, written in English. Inclusion was limited to articles published from 1995, as during the 1990s systematic reviews and 
meta-analyses started to replace narrative reviews, and methods and terminology were well established by that time. ${ }^{16}$ When the references could not be rejected with certainty, the full texts were obtained and a second screening was done. One article may report multiple MAs on different exposures.

The exposure of interest was any modifiable risk factor. 'Modifiable' was defined as a risk factor that is in any way reasonably modifiable, meaning that genetic and medical conditions were excluded as these are generally not modifiable. The outcome of interest was defined as incidence of bladder cancer as a binary outcome. Studies that included estimates based on prevalence or mortality were used as a proxy for incidence when estimates on incidence only were not available. Studies presenting results on genitourinary cancers without giving estimates for bladder cancer alone and studies reporting on urothelial cancer alone were excluded. Additionally, pooled risk estimates had to be presented with the accompanying 95\% confidence interval, accepted as odds ratio (OR), risk ratio (RR), hazard ratio (HR), standardised incidence ratio (SIR), or effect size (ES) if the latter consisted of a combination of the previously mentioned ratios. For the purpose of this paper, these estimates are all referred to as relative risks (RR).

\section{Data extraction}

Data was extracted by AA and all entries were double-checked completely for errors by KS. Any disagreement was discussed between the review authors until consensus was reached. When needed, authors of publications were contacted for additional information.

For each article, the following data was extracted when available: first author, year of publication, journal, risk factor(s) studied, number of events, types of studies included, number of studies included, type of risk estimate (OR, RR, SIR), outcome variable (incidence, prevalence, mortality, or combination), and per MA (statistical procedure): the exposures that were compared, the risk estimate and the $95 \%$ confidence interval, a measure of heterogeneity, the number of primary studies included, and a measure of publication bias.

\section{Selection criteria for estimates}

The estimates to be included had to be based on a minimum of two primary studies. They were selected based on the following list of decisions, and in this order: (1) bladder cancer only over urothelial cancers, (2) overall estimates over sub-group estimates, (3) more adjusted estimates over less adjusted estimates, (4) smoking adjusted over other adjustments, (5) incidence over prevalence or mortality, (6) random effects model over 
fixed effects model, and (7) pooling of cohort studies over pooling of case-control or cross-sectional studies.

\section{Synthesis of results}

If only one article was available on a specific risk factor, these estimates were included directly. If multiple articles were available, we selected the most comprehensive MA. This could occur in one of two ways. (1) The preferred method was to combine two MAs in a MMA to obtain an overall estimate based on the largest number of studies. However, we considered this only appropriate if the level of overlap between the primary studies included in each publication was no more than $50 \%$, measured as the percentage of studies of the smaller publication that overlapped with those of the larger publication. In addition, the exposure categories had to be comparable enough to be combined. (2) If, by following this rule, it was not possible to combine publications, the most comprehensive publication was included, based on the largest number of included primary studies. Basing this on the largest number of studies, rather than the largest sample size, was done because it was often not possible to determine the publication with the largest sample size due to insufficient data in the publication.

MMA was performed using a random-effects model. Heterogeneity was assessed between the included MAs using the $\mathrm{I}^{2}$ statistic. ${ }^{17}$ All statistical analyses were carried out in STATA V13.0 software and P values of 0.05 or below, or a confidence level of $95 \%$, were considered statistically significant.

In order to provide a measure of public health impact, a probability of causation ${ }^{18}$ (POC) was calculated for those risk estimates that were found to be significantly associated with the risk of bladder cancer. In addition, a combined $\mathrm{POC}^{18}$ was calculated for the lifestyle factors that one can be exposed to at one time. Careful attention was paid to avoid overlapping exposures, such as vitamin $\mathrm{C}$ intake, and fruit and vegetable consumption. In that case the most all-inclusive factor was included. No such combined estimate was calculated for all occupational factors combined, as one will unlikely be exposed to multiple occupations at one time.

POC can be interpreted as the percentage of exposed cases that are attributable to the exposure (e.g. the number of cases among smokers that are attributable to smoking). In case of a protective factor this should be read as the proportion of non-exposed cases that are attributable to the fact that they are non-exposed (e.g. the number of cases among people with low fruit and vegetable intake that are attributable to the low fruit and vegetable intake). 


\section{Role of the funding source}

The funding body was not involved in the study design; in the collection, analysis, or interpretation of data; in the writing of the report; or in the decision to submit the paper for publication.

\section{Results}

Figure 1 shows the literature search and selection process. A total of 1,496 articles were identified, from which 126 articles were selected based on the title and abstract for further evaluation. Of these, 32 articles were excluded (Appendix 1) for the following reasons: not being a MA, bladder cancer estimates were not reported separately from other urinary cancers, no risk estimate reported, or only mortality estimates were reported. Furthermore, 12 articles were excluded because all included primary studies were also part of a larger MA or because an update was available. This resulted in a total of 82 eligible articles (Appendix 2). Of these 82 articles, 21 articles were selected as the most comprehensive MA for a single risk factor, and five articles were combined in two MMAs, together reporting results for 36 risk factors. The statistically significant results from all most comprehensive MAs, totalling 19 lifestyle factors and 48 occupational factors, are summarised in table 1 and illustrated in figures 2 and 3, for the increased and decreased risks respectively.

\section{Lifestyle factors}

Four general topics emerged from the literature search with regard to lifestyle: dietary factors, consumption of tobacco, alcohol consumption, and physical activity.

\section{Dietary factors}

Fruit and vegetable

The MA by Yao et al.(2014) ${ }^{19}$ was selected as the most comprehensive publication for overall fruit and vegetable intake and reported significant protective effect on bladder cancer (RR 0.81; 95\%CI, 0.67-0.99; I2=58.4\%; $\mathrm{n}=10$ ).

\section{Fruit only}

MA by Yao et al.(2014) ${ }^{19}$ was selected as most comprehensive for consumption of overall fruit and the MA by Liang et al.(2014) ${ }^{20}$ for intake of citrus fruit (e.g. oranges, lemons, limes, and grapefruits). Overall fruit was associated with a reduced risk of bladder cancer (RR 0.77; 95\%CI, 0.69-0.87; I2=54.9\%; $\mathrm{n}=27$ ) as were citrus fruit (highest vs. lowest 


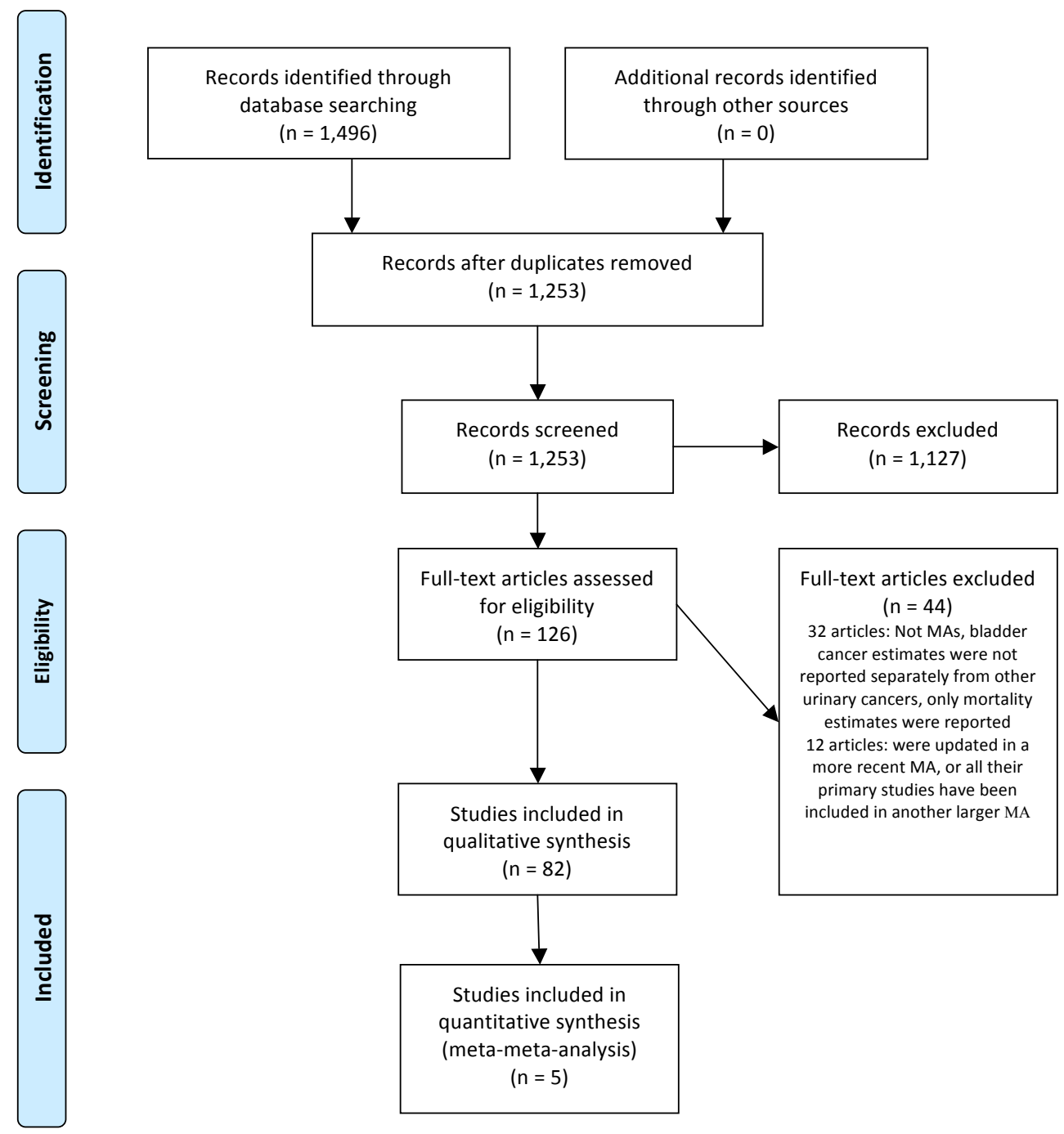

Fig. 1 Flow diagram of literature search and selection process

intake: RR 0.85; 95\% CI, 0.76-0.94; I2=72.1\%; n=14). Yao et al.(2014) ${ }^{19}$ reported significant publication bias for overall and citrus fruit intake.

\section{Vegetables only}

By pooling results from Yao et al.(2014) $)^{19}$ and Steinmaus et al.(2000) $)^{21}$ in a MMA, the most comprehensive estimate could be obtained for overall vegetable consumption ( $n=31$ unique and $n=3$ duplicate primary studies). The MA by Yao et al.(2014) ${ }^{19}$ was selected as the most comprehensive publication for cruciferous vegetables. Consumption 
of overall vegetables (RR $0.83 ; 95 \% \mathrm{CI}, 0.75-0.92 ; \mathrm{I} 2=0.0 \% ; \mathrm{n}=21+8)$ and cruciferous vegetables specifically (RR 0.84; 95\%CI, 0.77-0.91; $\mathrm{n}=11$ ), resulted in a reduced risk of bladder cancer.

\section{Total fluid}

Bai et al.(2014) ${ }^{22}$ was the most comprehensive estimate and found no statistically significant association between total fluid intake and bladder cancer (RR 1.12; 95\% CI, $0.94-1.33 ; \mathrm{I} 2=82.8 \% ; \mathrm{n}=14)$.

\section{Tea}

The MA by Qin et al.(2012) ${ }^{23}$ was selected as most comprehensive and they found a significant protective effect for black tea consumption (RR 0.79; 95\%CI, 0.59-0.99; $\mathrm{I} 2=33.1 \% ; \mathrm{n}=7$ ) but not for overall and green tea consumption.

\section{Coffee}

Six $\mathrm{MAs}^{24-28}$ reported on the association between coffee consumption and bladder cancer risk, of which Villanueva et al.(2006) ${ }^{27}$, Yu et al.(2011) $)^{26}$, and Wu et al.(2015) ${ }^{29}$ could be combined into a single estimate to provide the most comprehensive estimate ( $\mathrm{n}=$ 38 unique and $\mathrm{n}=3$ duplicate primary studies). No statistically significant association was observed, with substantial heterogeneity (RR 1.12; 95\%CI, 0.80-1.44; I2=94.2\%; $\mathrm{n}=18+9+6)$.

\section{Sweetened carbonated beverages}

Only one MA by Boyle et al.(2014) ${ }^{30}$ was identified, reporting on the association between sweetened carbonated beverage consumption and risk of bladder cancer. No statistically significant association was found (RR 1.13; 95\%CI, 0.89-1.45; $\mathrm{n}=5$ ).

\section{Milk and dairy products}

As reported by Mao et al.(2011) $)^{31}$, which was selected as most comprehensive, overall milk, skim milk, and fermented milk were associated with a significantly reduced incidence of bladder cancer (milk: RR 0.84; 95\%CI, 0.72-0.97; I2=70.1\%; $\mathrm{n}=16$; skim milk: RR 0.47; 95\% CI, 0.18-0.79; I2=0; $\mathrm{n}=2$; fermented milk: RR 0.69; 95\% CI, $0.47-0.91 ; \mathrm{I} 2=62.5 ; \mathrm{n}=5)$. In contrast, consumption of whole milk was associated with a significantly increased risk of bladder cancer (RR 2.23; 95\% CI, 1.45-3.00; I2=0; $\mathrm{n}=2$ ).

\section{Fish}

The MA by Li et al.(2011) ${ }^{32}$ was the only MA that studied the association between fish consumption and bladder cancer and reported no statistically significant association (RR 0.86; 95\%CI, 0.61-1.12). 
Chapter 5 I Modifiable risk factors for the prevention of bladder cancer

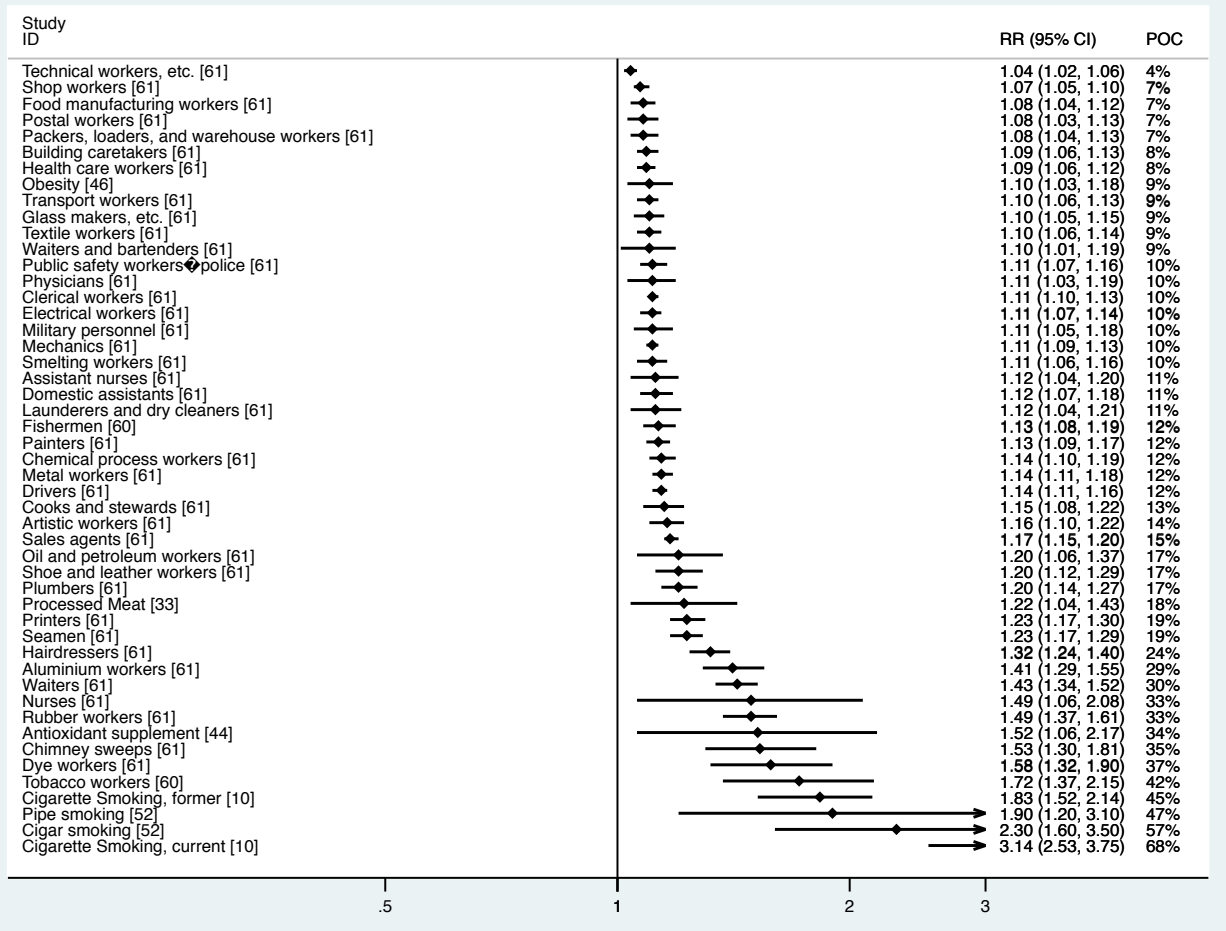

Fig. 2 Forest plot of significantly increased risks 
Study

ID

$\mathrm{RR}(95 \% \mathrm{Cl}) \quad \mathrm{POC}$

Selenium [42]

Vitamin A supplement [36]

Farmers [61]

Vitamin D [39]

Fruit consumption [19]

Gardeners [61]

Fruit and vegetable consumption [19]

Vitamin A [36]

Vitamin E [38]

Vegetable consumption [19,21]

Cruciferous vegetable [19]

Folate [41]

Citrus fruit [20]

Teachers [61]

Physical activity [56]

Forestry workers [61]

Religious and legal workers, etc. [61]

Economically inactive [61]

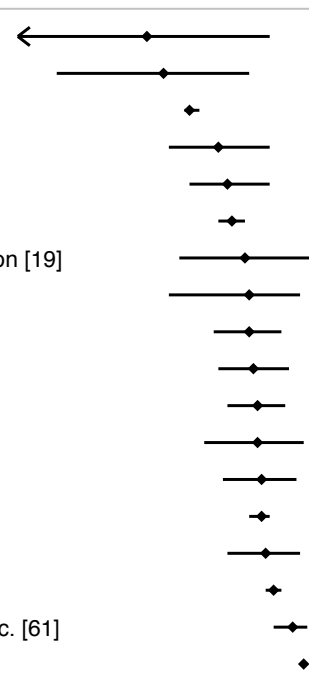

$0.61(0.42,0.87) 39 \%$

$0.64(0.47,0.82) 36 \%$

$0.69(0.68,0.71) 31 \%$

$0.75(0.65,0.87) 25 \%$

$0.77(0.69,0.87) 23 \%$

$0.78(0.75,0.81) 22 \%$

$0.81(0.67,0.99) 19 \%$

$0.82(0.65,0.95) 18 \%$

$0.82(0.74,0.90) 18 \%$

$0.83(0.75,0.92) 17 \%$

$0.84(0.77,0.91) 16 \%$

$0.84(0.72,0.96) 16 \%$

$0.85(0.76,0.94) 15 \%$

$0.85(0.82,0.87) 15 \%$

$0.86(0.77,0.95) 14 \%$

$0.88(0.86,0.90) 12 \%$

$0.93(0.88,0.97) 7 \%$

$0.96(0.95,0.97) 4 \%$

Fig. 3 Forest plot of significantly decreased risks 

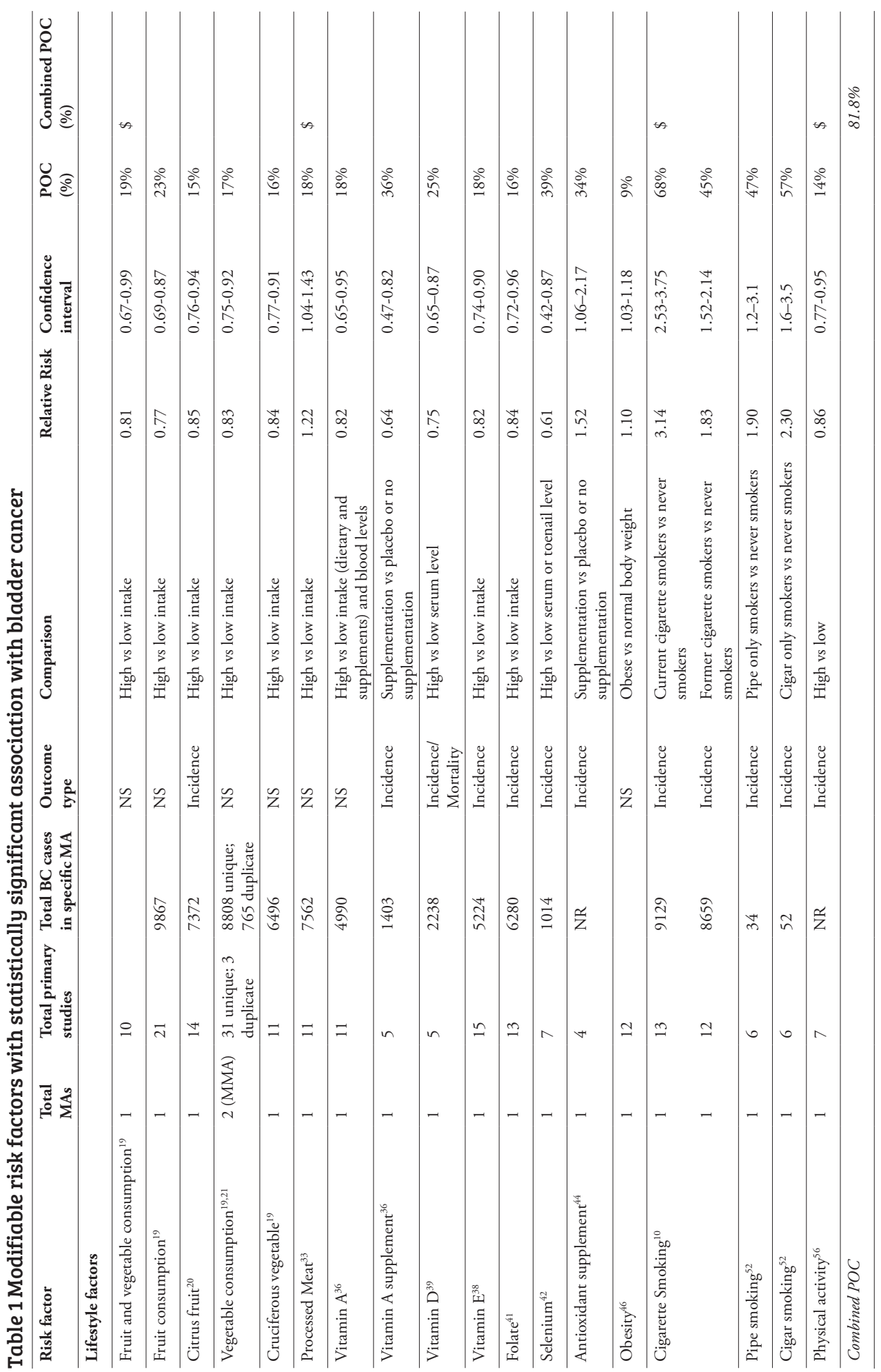


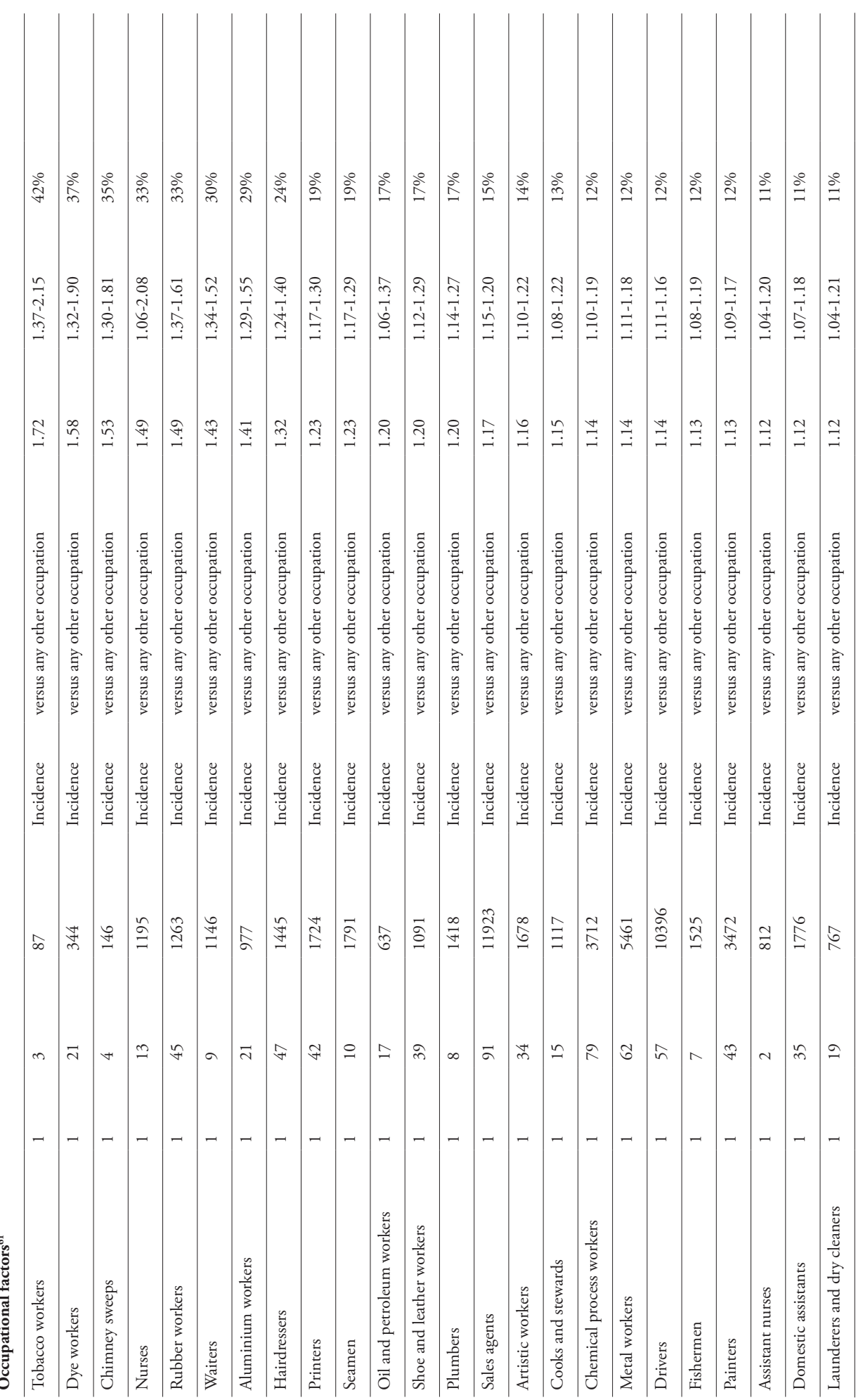




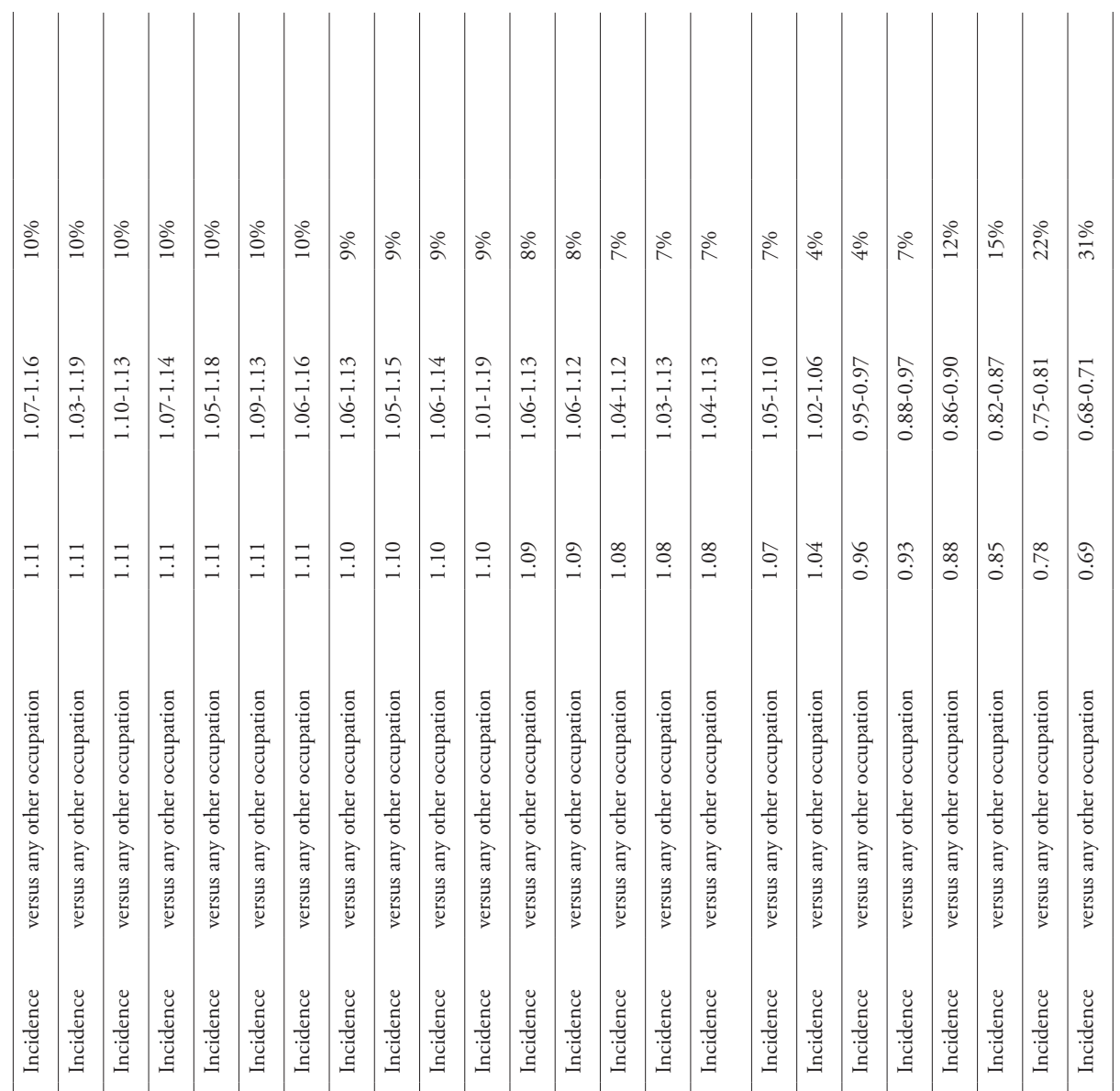

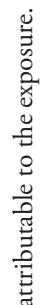

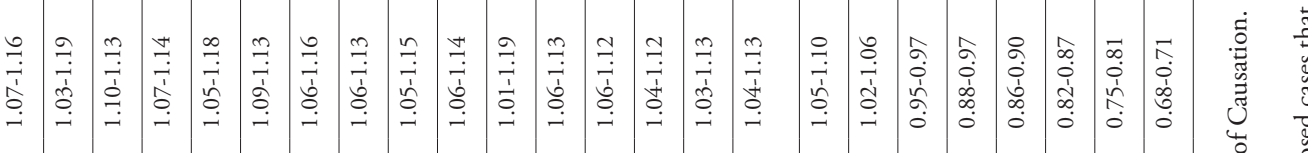

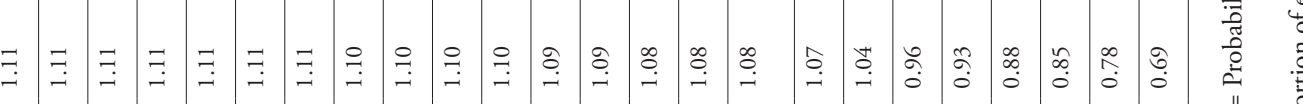

ప

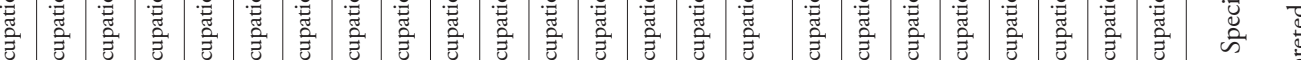

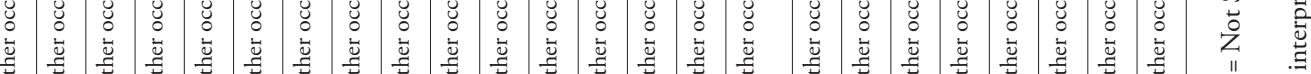

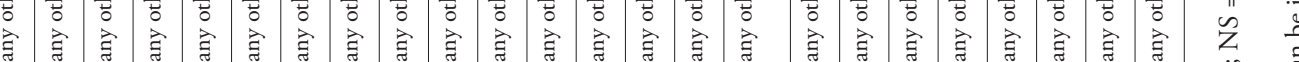

y

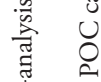

Е

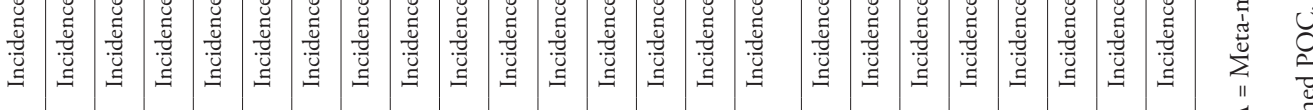

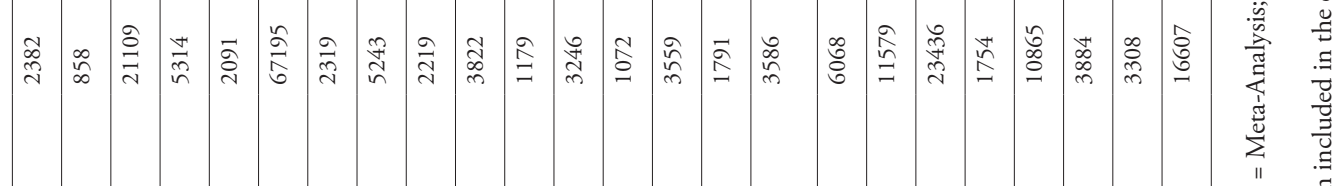

$\sum$ है:

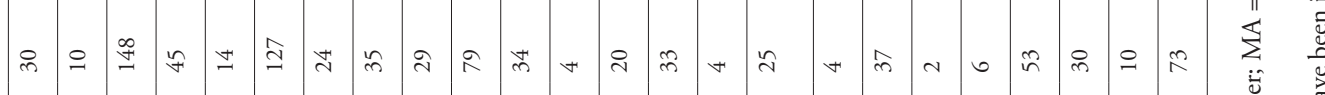

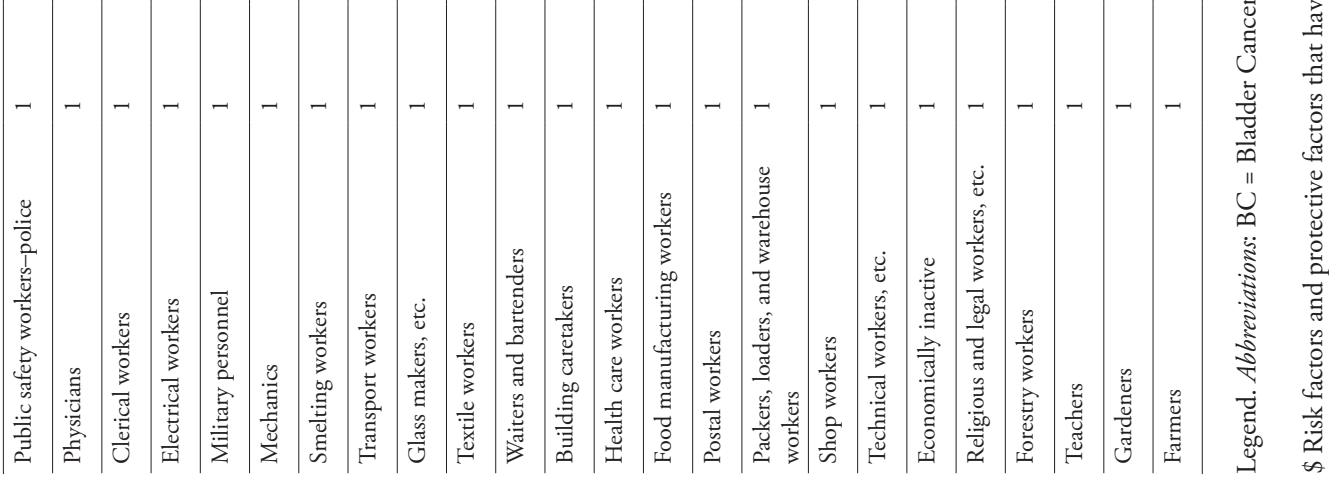




\section{Meat}

The MA by Li et al.(2014) ${ }^{33}$ was selected as most comprehensive and they found an increased risk of bladder cancer associated with processed meat (RR 1.22; 95\% CI, 1.041.43 ; $n=11$ ) but not with red meat (RR 1.15; 95\%CI, 0.97-1.36; $\mathrm{n}=14$ ).

\section{Alcohol}

The MA by Pelucchi et al.(2012) ${ }^{34}$ was considered most comprehensive and they found no statistically significant association for heavy drinkers ( $\geq 3$ drinks $(\geq 37.5 \mathrm{~g}) /$ day; RR 0.97; 95\%CI, 0.72-1.31; $\mathrm{n}=7$ ) or moderate drinkers ( $<3$ drinks/d; RR 0.98; 95\%CI, $0.89-1.07 ; \mathrm{n}=15)$.

\section{Egg}

The MA by Li et al.(2013) ${ }^{35}$ was the only MA identified in which no significant association was detected for overall egg consumption (RR 1.11; 95\%CI, 0.73-1.69; $\mathrm{n}=6$ ). However, when looking at cooking methods, an increased risk of bladder cancer was found for fried egg (RR 2.04; 95\%CI, 1.41-2.95; n=2) but not for boiled egg (RR 1.25 ; $95 \% \mathrm{CI}, 0.82-1.91 ; \mathrm{n}=2$ ).

\section{Vitamin A: body levels, intake, and supplementation}

The MA by Tang et al.(2014) $)^{36}$ was selected as most comprehensive MA for both overall intake and body levels of vitamin A, and vitamin A supplementation. They found a significantly lower risk of bladder cancer among individuals with higher dietary intake or higher blood levels of vitamin A (RR 0.82; 95\%CI, 0.65-0.95; I2=46.3\%; $\mathrm{n}=11$ ). When looking only at intake of vitamin $A$, the association was significantly protective for vitamin A supplementation, consisting of beta-carotene, and/or retinol supplementation (highest vs. lowest: RR 0.64; 95\%CI, 0.47-0.82; $\mathrm{I} 2=0 \%$ ) but not for dietary intake (highest vs. lowest: RR 0.90; 95\%CI, 0.80-1.01; I2=0\%).

Jeon et al.(2011) ${ }^{37}$ investigated the effect of only beta-carotene supplementation (so not retinol), compared to no supplementation, and found an increased risk of bladder cancer (RR 1.52; 95\%CI 1.03-2.24; I2=0.0\%; $\mathrm{n}=2$ ).

\section{Vitamin $C$}

The MA by Wang et al.(2014) $)^{38}$ was the only MA studying the association between vitamin $\mathrm{C}$ intake and bladder cancer. Combining vitamin $\mathrm{C}$ intake from diet and supplementation, they found a marginally significantly decreased risk of bladder cancer (RR 0.90; 95\%CI, 0.79-1.00; I2=43.7\%; $\mathrm{n}=20$ ). 


\section{Vitamin D}

The MA by Liao et al. ${ }^{39}$ investigated only the role of vitamin D serum level. They found that the risk of bladder cancer was decreased among individuals with the highest levels of serum vitamin D (RR 0.75; 95\%CI, 0.65-0.87; $n=5$ ). The MA by Chen et al. ${ }^{40}$ reported on the role of vitamin $\mathrm{D}$ intake from diet and supplements. Combining vitamin D intake from diet and supplementation, they found no significantly altered risk of bladder cancer (RR 0.92; 95\%CI, 0.66-1.28; $\mathrm{n}=3$ ).

\section{Vitamin E}

The MA by Wang et al. ${ }^{38}$ was selected as the most comprehensive MA and reported significantly reduced risk of bladder cancer for vitamin E intake (RR 0.82; 95\%CI, $0.74-0.90 ; \mathrm{I} 2=0 \% ; \mathrm{n}=15)$.

\section{Folate}

The association between folate intake and risk of bladder cancer was studied only in a MA by He et al.(2014) ${ }^{41}$. They found a significantly reduced risk of bladder cancer (RR 0.84; 95\%CI, 0.72-0.96; I2=28.9\%; $\mathrm{n}=13$ ). The association remained significantly reduced when pooling studies on dietary folate only (RR 0.82; 95\%CI, 0.65-0.99; $\mathrm{I} 2=57.9 \%$; $=9$ ), but not on supplemental folate only (RR 0.91; 95\%CI, 0.58-1.25; $\mathrm{I} 2=62.6 \% ; \mathrm{n}=3)$.

\section{Selenium: body levels and supplementation}

Two $\mathrm{MAs}^{42,43}$ were identified studying the association between selenium levels in the body, measured in serum or toenail, and bladder cancer. Amaral et al.(2011) ${ }^{42}$ was selected as the most comprehensive MA. They found that the risk of bladder cancer was significantly lower among individuals with the highest selenium levels in the body (RR $0.61 ; 95 \% \mathrm{CI}, 0.42-0.87 ; \mathrm{n}=7$ ).

Selenium supplementation was studied in one MA by Vinceti et al.(2014) ${ }^{43}$. They metaanalysed two RCTs and found no statistically significant association (RR 1.14; 95\% CI, $0.81-1.61 ; \mathrm{n}=2)$.

\section{Antioxidant supplementation}

One MA by Myung et al.(2010) ${ }^{44}$ reported on multiple types of antioxidant supplementation together, studied in RCTs. They found that antioxidant supplementation significantly increased the risk of bladder cancer (RR 1.52; 95\%CI 1.06-2.17; I2 $=0.0 \%$; $n=4)$. 


\section{Dietary acrylamide}

The association between dietary acrylamide intake and bladder cancer was studied in one MA, authored by Pelucchi et al.(2014) ${ }^{45}$ They reported no statistically significant association (RR 0.93; 95\%CI, 0.78-1.11; n=3).

\section{Obesity}

Two MAs ${ }^{46,47}$ reported on the association between body weight and bladder cancer. The MA by Sun et al. $(2015)^{46}$ was selected as most comprehensive. They found an increased risk of bladder cancer among obese individuals (BMI $\geq 30$; RR 1.10; 95\%CI, 1.03-1.18; $\mathrm{I} 2=8.8 \% ; \mathrm{n}=12$ ) but not among pre-obese individuals (BMI 25.00-29.99; RR 1.07; $95 \% \mathrm{CI}, 0.99-1.16 ; \mathrm{I} 2=46.1 \% ; \mathrm{n}=13)$.

\section{Consumption of tobacco}

Active smoking

Eight MAs ${ }^{10,11,48-53}$ were identified on the effect of active smoking of tobacco on the risk of bladder cancer. With regard to cigarette smoking, Cumberbatch et al.(2015) ${ }^{53}$ and Van Osch et al. $(2016)^{10}$ both included almost the same number of primary studies: $\mathrm{n}=90$ vs $\mathrm{n}=89$ respectively. Although theoretically this meant that the paper by Cumberbatch et al. would be most comprehensive, after discussion between the authors, the paper by Van Osch et al. was selected as most comprehensive as they reported dose-response analyses on duration, intensity, and time since cessation of smoking. Because smoking was already known to be the most important modifiable risk factor of bladder cancer, the dose-response variables were considered valuable additional information. It must be noted that calculated risks do not differ much between both MAs (Appendix 2).

Van Osch et al.(2016) ${ }^{10}$ reported a significantly increased risk of more than 3 times in current smokers (RR 3.14; 95\%CI, 2.53-3.75) and almost 2 times in former smokers (RR 1.83; 95\% CI, 1.52-2.14). Their dose-response associations showed a positive trend with increasing intensity of smoking and number of pack years, reaching a plateau from about 15 cigarettes/day and 50 pack-years. Increasing duration since cessation of smoking resulted in a reducing risk, although former smokers remained at a 50\% increased risk even after more than 20 years of cessation.

Hemelt et al.(2009) ${ }^{11}$ also reported on the risk among smokers of any type of tobacco. They found an increased incidence of bladder cancer among current smokers (RR 3.35; 95\%CI, 2.90-3.88; $\mathrm{n}=11$ ) as well as among ever-smokers (RR 2.25; 95\%CI, 1.962.59; $\mathrm{n}=15)$.

Two MAs ${ }^{52,53}$ additionally reported on the effect of pure cigar and pure pipe smoking. 
Pitard et al.(2001) $)^{52}$ was considered most comprehensive and they reported a significantly increased risk of bladder cancer among both pipe smokers (RR 1.9; 95\%CI, 1.2-3.1; $\mathrm{n}=6$ ) and cigar smokers (RR 2.3; 95\%CI, 1.6-3.5; n=6).

\section{Passive smoking}

Van Hemelrijck et al.(2009) ${ }^{54}$ reported on the association between passive smoking and bladder cancer. They found no statistically significant association in individuals exposed to passive smoking (RR 0.99; 95\%CI, 0.86-1.14; I2=35.6\%; $\mathrm{n}=8$ ) compared to neversmoking individuals with no exposure to passive smoking.

\section{Smokeless Tobacco}

Use of smokeless tobacco, including chewing tobacco, oral snuff, and unspecified smokeless tobacco, was studied in two MAs..$^{53,55}$ Lee et al.(2009) ${ }^{55}$ was considered most comprehensive. No statistically significant association was found with development of bladder cancer (RR 0.95; 95\%CI, 0.71-1.29; n=9).

\section{Physical activity}

Keimling et al.(2014) ${ }^{56}$ wrote the only MA on the association between physical activity and bladder cancer. They found a statistically significant protective effect on the bladder cancer risk among individuals with the highest levels of physical activity (RR 0.86; 95\%CI, 0.77-0.95; n=7).

\section{Personal hair dye use}

Turati et al. $(2014)^{57}$ updated previous MAs $s^{58-60}$ to investigate the association between personal use of hair dye and bladder cancer. They found no statistically significant increased risk among users of personal hair dye (smoking adjusted RR 0.94; 95\%CI, $0.82-1.08 ; \mathrm{n}=12)$.

\section{Occupational factors}

Multiple MAs were identified on the association between occupational factors and bladder cancer. Most MAs reported on a single occupation or occupational group, or a single specific occupational exposure (Appendix 2). Moreover, four MAs ${ }^{61-64}$ reported on a large number of occupational categories. Cumberbatch et al.(2015) ${ }^{61}$ and Reulen et al.(2008) $)^{62}$ authored two large MAs on different occupations based on 217 and 130 different studies respectively, whereas Kogevinas et al.(2003) ${ }^{63}$ and 't Mannetje et al.(1999) ${ }^{64}$ both did a pooled analysis of eleven case-control studies conducted in European countries reporting results for men and women, respectively. The paper by Cumberbatch et al. was considered the most comprehensive for all identified occupations based on the number of studies included. They found the following occupations to 
have a statistically significant increased risk of more than $20 \%$ : tobacco workers, dye workers, chimney sweeps, nurses, rubber workers, waiters, aluminium workers, hairdressers, printers, seamen, oil and petroleum workers, shoe and leather workers, and plumbers. Protective effects were found for farmers, gardeners, teachers, forestry workers, religious and legal workers, and economically inactive workers. Statistically significant associations reported by Cumberbatch et al. are listed in Table 1. All other associations are summarized in appendix 3 along with findings by Reulen et al.(2008) ${ }^{62}$, Kogevinas et al.(2003) ${ }^{63}$, and 't Mannetje et al.(1999). ${ }^{64}$

\section{Probability of Causation}

The estimates for probability of causation (POC), displayed in percentages, are presented in table 1 . The percentages ranged from $9 \%$ to $68 \%$ among the lifestyle factors, and between $4 \%$ and $42 \%$ for the occupational exposures. The four highest POCs are found among the smoking estimates: $68 \%$ of the bladder cancer incidence among cigarette smokers, $57 \%$ among cigar smokers, $47 \%$ among pipe smokers and $45 \%$ among former cigarette smokers can be attributed to this habit.

A POC was calculated for four factors combined, which were considered sufficiently independent: total fruit and vegetable consumption, processed meat consumption, smoking, and physical activity. To avoid overlap, the only estimate included for smoking was for cigarette smoking as most smokers are (also) cigarette smokers (78.7\%) and only $4.5 \%$ was found to be pure pipe and/or cigar smoker. ${ }^{52}$ The combined POC showed that up to $81.8 \%$ of the bladder cancer cases, among those with non-optimal lifestyle behaviours, could be prevented through lifestyle modifications.

\section{Discussion}

In total, 12 lifestyle factors and 48 occupational factors emerged that significantly increased or decreased the risk of bladder cancer. Such numerous modifiable risk factors present an opportunity and great potential for prevention. Below, the significantly associated risk factors will be discussed.

\section{Smoking}

The most important risk factor proved to be smoking, particularly the risk was highest for current smokers of cigarettes (RR 3.14), ${ }^{10}$ but also for smokers of cigars only (RR 2.3) and pipes only (RR 1.9). ${ }^{52}$ An interesting finding was that a plateau was reached at about 15 cigarettes/day, meaning that heavier smoking does not carry a much higher risk than less heavy smoking. ${ }^{10}$ Although the risk slowly decreased with longer cessation 
of cigarette smoking, the RR was still 50\% increased 20 years of cessation.

Characteristics of the tobacco smoked may influence this association. In previous studies, individuals smoking black tobacco had a higher risk compared to smokers of blond tobacco, due to the higher concentrations of carcinogens in black tobacco. ${ }^{65-71}$ Also, inhalation of the tobacco smoke into the lungs and throat resulted in a higher risk compared to inhalation only into the mouth, ${ }^{49,65,71-76}$ although not all evidence supports this. $^{.77-80}$

Tobacco smoke contains numerous carcinogens that contribute to the initiation and promotion of tumour development. These chemicals are renally excreted, making it directly toxic to the human urinary bladder. During metabolisation of these compounds, DNA-adducts are formed, leading to permanent genetic mutations. If this occurs in an oncogene or tumour suppressor gene, it may result in uncontrolled growth and eventually cancer.

Genetic mutations, such as NAT2 slow acetylation and GSTM null genotypes, have previously been shown to be associated with increased susceptibility to cancer. ${ }^{3}$ The enzymes for which the genes code play a role in the detoxification pathways of i.a. aromatic amines and PAHs. These mutations do not intrinsically cause bladder cancer but do increase susceptibility when exposed to tobacco smoke or other sources of exposure. ${ }^{81,82}$

\section{Dietary factors}

Because the metabolites of many food groups are excreted by the urinary tract, dietary factors have often been suggested and researched as risk factors of bladder cancer. ${ }^{83}$ Several different nutritional factors have been identified, showing both increased and decreased risks of bladder cancer.

\section{Fruit, vegetables, and antioxidants}

One key group of foods that was associated with a lower risk of bladder cancer were fruits and vegetables. Besides these groups as a whole, intake of both citrus fruit and cruciferous vegetables were researched. The effect is suggested to be due to the vitamins, minerals, and phytochemicals that fruit and vegetables contain, which have antioxidant properties. ${ }^{84,85}$ Antioxidants may protect against cancer through inhibition of oxidation of DNA, controlling of cell proliferation and apoptosis ${ }^{85}$, and facilitation of metabolisation of carcinogenic compounds into less toxic substances, although the association may be dose-dependent. ${ }^{86}$ 
It should be noted that significant publication bias was found in the MA on overall and citrus fruit consumption. ${ }^{19}$ Because small studies with negative results tend not to be published, the effect size found in the MAs on these risk factors may actually be attenuated.

The MAs included in our review that studied vitamins separately, came to supporting conclusions. For all but vitamin $\mathrm{C}$, higher intakes were associated with a significantly reduced risk of bladder cancer, with effects on $-39 \%$ for selenium, $-18 \%$ for vitamin A and $\mathrm{E}$, and $-16 \%$ for folate.

Vitamin A intake was found to be negatively associated with bladder cancer when data on dietary intake, supplementation, and blood levels were combined. However, when studied by intake source separately, the protective effect was only found for vitamin A supplementation but not for dietary intake. ${ }^{36}$ Furthermore results on supplementation were not consistent as Jeon et al.(2011) ${ }^{37}$, found a significantly increased risk of bladder cancer of $52 \%$ among participants taking supplementation in experimental studies. These conflicting results may be the result of a dose-dependent relationship, showing intake to be protective at low doses and harmful at high doses. Another explanation may be the different study design, in which the reduced risk found in observational studies may actually be the effect of an overall healthier lifestyle, as those individuals who choose to use nutritional supplementation tend to have a healthier lifestyle overall. ${ }^{87}$ Vitamin A plays an important role in cell proliferation and differentiation ${ }^{88}$, and carotenoids can directly affect carcinogenesis through their antioxidant properties, or indirectly after transformation of provitamin A carotenoids into vitamin A. ${ }^{89,90}$

Vitamin E consists of a group of lipid soluble molecules, including four tocopherols and four tocotrienols. Specifically $\gamma$-tocopherol, $\delta$-tocopherol, and tocotrienols inhibit several inflammatory pathways ${ }^{91}$, which is important because chronic inflammation or chronic infection influences development and progression of cancer ${ }^{92}$, In addition, vitamin $\mathrm{E}$ is a potent lipid-soluble antioxidant, inhibiting lipid peroxidation and thereby protecting cell membranes from peroxidative damage. ${ }^{93}$

Although higher levels of selenium protected against bladder cancer, causation could not be confirmed through supplementation intervention. ${ }^{42}$ The specific mechanism of action of selenium in carcinogenesis is not yet well understood. Several theories have been suggested, including selenoproteins serving as antioxidants and metabolites of selenium involved in redox cycling, modification of protein thiols, and methionine mimicry. ${ }^{94}$ 
However, despite these suggested protective properties of antioxidants, analysis of multiple types of antioxidant supplementation together, showed a $52 \%$ increased risk of bladder cancer. ${ }^{44}$ An explanation for this discrepancy may be that it is not one antioxidant alone that has cancer-preventative properties, but rather the interaction between multiple antioxidants and other phytochemicals, such as it occurs with consumption of a diet rich in fruit and vegetables.

\section{Other nutrients and food groups}

Because consumption of processed meat but not red meat was associated with bladder cancer, the effect may result from different forms of mechanical, chemical, and enzymatic treatment that processed meat has been exposed to. One hypothesis suggests that nitrite, which is used as a colour and flavour preservative in processed meat, combines with secondary amines from proteins to form nitrosamines that are found to be carcinogenic to, amongst other organs, the bladder. ${ }^{95}$ Although red and processed meat are often studied in relation to disease development, particularly cancer, the exact mechanism of action is still unclear and is likely to be a combination of factors present in or associated with meat consumption. ${ }^{96,97}$

The protective effect of folate, a vitamin predominantly found in vegetables, is suggested to be through its role in DNA synthesis, repair, and methylation. ${ }^{98}$ In previous research, low levels of folate were associated with hypomethylation of DNA resulting in dysregulation of proto-oncogenes and tumour suppressor genes, and therefore an increased risk of cancer. ${ }^{99}$ However, the association is not as straightforward as may be expected. Research suggests that timing and dose of folate plays an important role in its effect on cancer development. ${ }^{100}$ Because folate plays a role in de-novo synthesis of nucleotides, it will support healthy tissue and prevent tumour development. However, once pre-neoplastic lesions have been established, it will also support these rapidly proliferating tissues resulting in rapid tumour progression.

In line with previous findings for colorectal cancer ${ }^{101}$ and breast cancer, ${ }^{102}$ higher serum vitamin $\mathrm{D}$ was found to be protective against bladder cancer. Calcitriol, the potent hormone produced in the body from vitamin $\mathrm{D}$, plays a role in multiple stages of cancer development, through both genomic and non-genomic pathways. ${ }^{103}$ No association was found for dietary and supplemental intake of vitamin $\mathrm{D}$, which may result from dietary intakes not adequately reflecting calcitriol available to the body as it is also endogenously formed in response to UV-light.

Previous reviews have also mentioned other dietary factors increasing the risk of bladder cancer, among which higher intake of soy, fat, barbecued meats, and artificial 
sweeteners. ${ }^{84,104}$ However, no MAs were identified on these factors so these factors have not been included in our overview.

\section{Fluid intake and water contaminants}

Whereas increased intake of fluids dilutes urine and increases micturition, leading to a reduced exposure of the bladder to carcinogens, it may also increase exposure when the fluid itself contains carcinogens. ${ }^{3}$ For example, increased risk of bladder cancer was found with water chlorination ${ }^{105,106}$ and arsenic contamination. ${ }^{107,108}$ However, no significant association was found for either all fluid intake or water specifically. ${ }^{22}$ Together, this is in line with the suggested dual effect of increased fluid intake on bladder cancer risk.

\section{Physical activity}

Physical activity was found to have a small but significant protective effect against bladder cancer. The effect found was not modified by BMI or smoking, as the authors explain in their MA. ${ }^{56}$ Although the exact mechanism through which physical activity protects against cancer is not yet known, several mechanisms have been suggested acting on both tumour initiation and progression. These potential mechanisms include modification of carcinogen activity, and enhancement of antioxidant and DNA repair processes. ${ }^{109}$

\section{Occupation and occupational exposure to chemicals}

Occupational factors are considered the second most important risk factor for bladder cancer after smoking. ${ }^{3,110}$ Over the years, many carcinogens have been identified by the IARC as definitely (group 1) or probably carcinogenic (group 2a). Occupational exposure to these carcinogens has been largely controlled and workers are now exposed to weaker carcinogens. The results of the MA by Cumberbatch et al.(2015) ${ }^{53}$ show multiple occupations or occupational exposures that are significantly associated with the risk of bladder cancer.

Particularly workers exposed to aromatic amines, PAH, tobacco and tobacco smoke, combustion products, and heavy metals are at an increased risk. However, these findings will not be further discussed and repeated here as the MA by Cumberbatch et al.(2015) ${ }^{61}$ offers an interesting and elaborate discussion on the matter. They also attempted to study a change in occupational risk over time but were limited by heterogeneity and small sample sizes.

Interestingly, farmers, gardeners, teachers, and forestry workers were found to be at a significantly lower risk. Farmers have been studied numerous times with regard to disease risk and have been suggested to be at both an increased and decreased risk of bladder cancer. It is thought of as a risk factor due to the exposure to chemicals such 
as pesticides, viruses, and other exposures. Although an increased risk was found in association with exposure to some pesticides, the evidence is not conclusive. ${ }^{111}$ Both the European Food Safety Authority (EFSA) ${ }^{112}$ and the IARC ${ }^{113}$ did not find sufficient evidence to support carcinogenicity of pesticides. On the other hand, farmers, and possibly also gardeners and forestry workers, often have higher physical activity levels and fruit and vegetable consumption, as well as a lower prevalence of smoking. ${ }^{111}$

Finally, in addition to exposure to carcinogens on an occupational basis, an increased risk of bladder cancer in certain occupational groups may also be the result of fewer possibilities for micturition during working hours. This may apply to, for example, sales workers, professional drivers, or other occupations where bathroom visits are limited to break times.

\section{Public health impact}

The calculated POCs show that by adopting the right lifestyle, while considering harmful environmental and occupational exposures, a large proportion of the burden of bladder cancer could be prevented. Particularly for smoking the POC was high, highlighting that smoking cessation and prevention of smoking initiation should remain high on the agenda. In addition, a diet rich in fruit and vegetables, particularly citrus fruit and cruciferous vegetables, and low in processed meat should be stimulated. Particularly working conditions involving exposure to aromatic amines, PAHs, tobacco and tobacco smoke, combustion products, and heavy metals should be given priority to reduce exposure to these compounds.

\section{Limitations}

The quality of a MA is strongly determined by the quality of the primary studies included. Not all studies corrected for smoking status, which is the most important risk factor for bladder cancer. The effects of this may be large, leading to an under- or overestimation of the true effect size. To minimise the effect of confounding in our study, only the most adjusted estimates from each MA were selected. Selection of the most comprehensive publication was based on the largest number of primary studies included, rather than the largest sample size. The latter would have been more desirable as the power of a study depends on it, but this data was often unavailable in the publications and number of studies included had to be used as a proxy. Results of MMA could be affected by duplicate inclusion of primary studies, leading to inflated precision and homogeneity. ${ }^{114}$ Therefore, we minimised duplicate inclusion and performed a MMA only if at least $50 \%$ of the included primary studies were 'new' in subsequent MAs.

The main limitation with regard to the combined POC is assumption of independence 
of risk factors. It is well known that lifestyle behaviours tend to cluster ${ }^{115}$, indicating that the factors are unlikely to be fully independent. Therefore, the combined POC is likely to be an overestimation and should be considered a maximum. However, the POCs of each of the individual factors are substantial, indicating that each plays an important part in the risk.

In this study, estimates of bladder cancer incidence were given priority over estimates of prevalence or mortality. Although the majority of included studies specified the outcome measure that was included or provided stratified results, not all did (Table 1). Because mortality and prevalence figures are greatly influenced by treatment success, this may have influence the estimates obtained.

Heterogeneity may always be an issue in MAs. Several of the studies included in the current review show moderate to high levels of heterogeneity. Whereas some identified study design, gender, or geographical region as possible source of heterogeneity ${ }^{19,22,31}$, others could not find an explanation. ${ }^{20}$

It should be noted that different classification criteria may be applied in studies to determine cases, including the TNM classification and stage grouping. Because any effect would be unlikely and small, this potential difference was not taken into consideration when selecting estimates.

This publication is a review of MAs. Additional risk factors for which no MA is available may prove to be of importance, but have not been included in this review. This study will help to identify gaps in knowledge with regard to MAs available.

It was our responsibility to give an objective and complete overview of the literature available. By using a protocol, including only MAs and by dealing appropriately with unexpected issues, we believe to have provided an overview of the risk factors associated with bladder cancer with high levels of objectivity and reliability.

In conclusion, the burden of bladder cancer could be significantly reduced through modification of lifestyle, environment, and occupational exposures. In fact, having numerous modifiable risk factors, among which multiple with a substantial RR, makes bladder cancer one of the most preventable diseases. Although substantial risks were found for, for example, smoking, some identified risk factors 'only' led to an increase or decrease of $10-15 \%$. This could be considered a minor effect, but reducing prevalence of a number of smaller risk factors together, could still result in significant overall risk reductions in the general population. Theoretically the included risk factors are, at least 
partially, modifiable. However, actually changing them is not always easily achieved, neither for one individual, let alone on a population level. Therefore, it is important that preventative and protective strategies pay attention to behaviour change in order to capture the greatest potential of risk reduction. When change is achieved, impact will not remain restricted to an improvement in the incidence of bladder cancer, as most of the included risk factors also play a role the risk of multiple other chronic diseases such as cancer or cardiovascular diseases, ${ }^{116-118}$ and deaths. ${ }^{119}$ Since relapse of bladder cancer is common, future research may also elucidate whether the same risk factors play a role in prevention of bladder cancer relapse as they do in incidence.

\section{Acknowledgments}

This project was funded by the Maastricht University Interfaculty Programme 'Eatwell'. The funder had no influence in the development and execution of this systematic review.

\section{Conflict of interest}

The authors declare that they have no conflict of interest. 


\section{References}

1. Cancer IA for R on. GLOBOCAN 2012: estimated cancer incidence, mortality and prevalence worldwide in 2012. World Heal. Organ. http//globocan. iarc. fr/Pages/fact_sheets_cancer. aspx. Accessed. 2014;9.

2. Czene K, Lichtenstein P, Hemminki K. Environmental and heritable causes of cancer among 9.6 million individuals in the Swedish family-cancer database. Int. J. Cancer. 2002;99:260-6.

3. Burger M, Catto JWF, Dalbagni G, Grossman HB, Herr H, Karakiewicz P, et al. Epidemiology and risk factors of urothelial bladder cancer. Eur. Urol. 2013;63:234-41.

4. Chavan S, Bray F, Lortet-Tieulent J, Goodman M, Jemal A. International variations in bladder cancer incidence and mortality. Eur. Urol. 2013;

5. Jemal A, Bray F, Center MM, Ferlay J, Ward E, Forman D. Global cancer statistics. CA. Cancer J. Clin. 2011;61:69-90.

6. Zeegers MPA, Kellen E, Buntinx F, van den Brandt PA. The association between smoking, beverage consumption, diet and bladder cancer: a systematic literature review. World J. Urol. 2004;21:392401.

7. Vineis P, Wild CP. Global cancer patterns: causes and prevention. Lancet. 2014;383:549-57.

8. Rushton L, Bagga S, Bevan R, Brown TP, Cherrie JW, Holmes P, et al. Occupation and cancer in Britain. Br. J. Cancer. 2010;102:1428-37.

9. Ng M, Freeman MK, Fleming TD, Robinson M, Dwyer-Lindgren L, Thomson B, et al. Smoking prevalence and cigarette consumption in 187 countries, 1980-2012. Jama. 2014;311:183-92.

10. van Osch FHM, Jochems SHJ, van Schooten F-J, Bryan RT, Zeegers MPA. Quantified relations between exposure to tobacco smoking and bladder cancer risk: a meta-analysis of 89 observational studies. Int. J. Epidemiol. 2016;

11. Hemelt M, Yamamoto H, Cheng KK, Zeegers MPA. The effect of smoking on the male excess of bladder cancer: a meta-analysis and geographical analyses. Int. J. Cancer. 2009;124:412-9.

12. Clark PE, Agarwal N, Biagioli MC, Eisenberger MA, Greenberg RE, Herr HW, et al. Bladder cancer. J. Natl. Compr. Canc. Netw. 2013;11:446-75.

13. Yeung C, Dinh T, Lee J. The health economics of bladder cancer: an updated review of the published literature. Pharmacoeconomics. 2014;32:1093-104.

14. Al-Zalabani A, Stewart K, Wesselius A, Zeegers M. Modifiable risk factors for the prevention of bladder cancer: a systematic review of meta-analyses. PROSPERO 2015:CRD42015023411 [Internet]. Available from: http://www.crd.york.ac.uk/PROSPERO/display_record. asp?ID=CRD42015023411

15. Moher D, Liberati A, Tetzlaff J, Altman DG. Preferred reporting items for systematic reviews and meta-analyses: the PRISMA statement. BMJ. 2009;339:b2535.

16. Borenstein M, Hedges L V., Higgins JPT, Rothstein HR. Front Matter. Introd. to Meta-Analysis. Chichester, UK: John Wiley \& Sons, Ltd; 2009.

17. Higgins JPT, Thompson SG, Deeks JJ, Altman DG. Measuring inconsistency in meta-analyses. BMJ. 2003;327:557-60.

18. Bouter LM, Dongen MCJM van, Zielhuis GA, Zeegers MP. Leerboek epidemiologie. Houten,the Netherlands: Bohn Stafleu van Loghum; 2015.

19. Yao B, Yan Y, Ye X, Fang H, Xu H, Liu Y, et al. Intake of fruit and vegetables and risk of bladder cancer: a dose-response meta-analysis of observational studies. Cancer Causes Control. 2014;

20. Liang S, Lv G, Chen W, Jiang J, Wang J. Citrus fruit intake and bladder cancer risk: a meta-analysis of observational studies. Int. J. Food Sci. Nutr. 2014;1-6.

21. Steinmaus CM, Nuñez S, Smith AH. Diet and bladder cancer: a meta-analysis of six dietary variables. Am. J. Epidemiol. 2000;151:693-702.

22. Bai Y, Yuan H, Li J, Tang Y, Pu C, Han P. Relationship between bladder cancer and total fluid intake: a meta-analysis of epidemiological evidence. World J. Surg. Oncol. England; 2014;12:223.

23. Qin J, Xie B, Mao Q, Kong D, Lin Y, Zheng X. Tea consumption and risk of bladder cancer: a 
meta-analysis. World J. Surg. Oncol. England; 2012;10:172.

24. Huang T, Guo Z, Zhang X, Zhang X, Liu H, Geng J, et al. Coffee consumption and urologic cancer risk: a meta-analysis of cohort studies. Int. Urol. Nephrol. 2014;46:1481-93.

25. Zhou Y, Tian C, Jia C. A dose-response meta-analysis of coffee consumption and bladder cancer. Prev. Med. (Baltim). 2012;55:14-22.

26. Yu X, Bao Z, Zou J, Dong J. Coffee consumption and risk of cancers: a meta-analysis of cohort studies. BMC Cancer. England; 2011;11:96.

27. Villanueva CM, Cantor KP, King WD, Jaakkola JJK, Cordier S, Lynch CF, et al. Total and specific fluid consumption as determinants of bladder cancer risk. Int. J. Cancer. 2006;118:2040-7.

28. Sala M, Cordier S, Chang-Claude J, Donato F, Escolar-Pujolar A, Fernandez F, et al. Coffee consumption and bladder cancer in nonsmokers: a pooled analysis of case-control studies in European countries. Cancer Causes Control. 2000;11:925-31.

29. Wu W, Tong Y, Zhao Q, Yu G, Wei X, Lu Q. Coffee consumption and bladder cancer: a metaanalysis of observational studies. Sci. Rep. Macmillan Publishers Limited. All rights reserved; 2015;5.

30. Boyle P, Koechlin A, Autier P. Sweetened carbonated beverage consumption and cancer risk: metaanalysis and review. Eur. J. Cancer Prev. 2014;23:481-90.

31. Mao Q-Q, Dai Y, Lin Y-W, Qin J, Xie L-P, Zheng X-Y. Milk consumption and bladder cancer risk: a meta-analysis of published epidemiological studies. Nutr. Cancer. 2011;63:1263-71.

32. Li Z, Yu J, Miao Q, Sun S, Sun L, Yang H, et al. The association of fish consumption with bladder cancer risk: a meta-analysis. World J. Surg. Oncol. 2011;9:107.

33. Li F, An S, Hou L, Chen P, Lei C, Tan W. Red and processed meat intake and risk of bladder cancer: a meta-analysis. Int. J. Clin. Exp. Med. 2014;7:2100-10.

34. Pelucchi C, Galeone C, Tramacere I, Bagnardi V, Negri E, Islami F, et al. Alcohol drinking and bladder cancer risk: a meta-analysis. Ann. Oncol. 2012;23:1586-93.

35. Li F, Zhou Y, Hu R, Hou L, Du Y-J, Zhang X, et al. Egg consumption and risk of bladder cancer: a meta-analysis. Nutr. Cancer. 2013;65:538-46.

36. Tang J-E, Wang R-J, Zhong H, Yu B, Chen Y. Vitamin A and risk of bladder cancer: a meta-analysis of epidemiological studies. World J. Surg. Oncol. 2014;12:130.

37. Jeon Y-J, Myung S-K, Lee E-H, Kim Y, Chang YJ, Ju W, et al. Effects of beta-carotene supplements on cancer prevention: meta-analysis of randomized controlled trials. Nutr. Cancer. 2011;63:1196207.

38. Wang Y-Y, Wang X-L, Yu Z-J. Vitamin C and E intake and risk of bladder cancer: a meta-analysis of observational studies. Int. J. Clin. Exp. Med. 2014;7:4154-64.

39. Liao Y, Huang J-L, Qiu M-X, Ma Z-W. Impact of serum vitamin D level on risk of bladder cancer: a systemic review and meta-analysis. Tumour Biol. 2014;

40. Chen F, Li Q, Yu Y, Yang W, Shi F, Qu Y. Association of vitamin C, vitamin D, vitamin E and risk of bladder cancer: a dose-response meta-analysis. Sci. Rep. Nature Publishing Group; 2015;5:9599.

41. He H, Shui B. Folate intake and risk of bladder cancer: a meta-analysis of epidemiological studies. Int. J. Food Sci. Nutr. 2014;65:286-92.

42. Amaral AFS, Cantor KP, Silverman DT, Malats N. Selenium and bladder cancer risk: a metaanalysis. Cancer Epidemiol. Biomarkers Prev. 2010;19:2407-15.

43. Vinceti M, Dennert G, Crespi CM, Zwahlen M, Brinkman M, Zeegers MPA, et al. Selenium for preventing cancer. Cochrane database Syst. Rev. 2014;3:CD005195.

44. Myung S-K, Kim Y, Ju W, Choi HJ, Bae WK. Effects of antioxidant supplements on cancer prevention: meta-analysis of randomized controlled trials. Ann. Oncol. 2010;21:166-79.

45. Pelucchi C, Bosetti C, Galeone C, La Vecchia C. Dietary acrylamide and cancer risk: an updated meta-analysis. Int. J. Cancer. 2015;136:2912-22.

46. Sun J-W, Zhao L-G, Yang Y, Ma X, Wang Y-Y, Xiang Y-B. Obesity and risk of bladder cancer: a dose-response meta-analysis of 15 cohort studies. PLoS One. 2015;10:e0119313.

47. Qin Q, Xu X, Wang X, Zheng X-Y. Obesity and risk of bladder cancer: a meta-analysis of cohort 
studies. Asian Pac. J. Cancer Prev. 2013;14:3117-21.

48. Puente D, Hartge P, Greiser E, Cantor KP, King WD, Gonzalez CA, et al. A pooled analysis of bladder cancer case-control studies evaluating smoking in men and women. Cancer Causes Control. Netherlands; 2006;17:71-9.

49. Freedman ND, Silverman DT, Hollenbeck AR, Schatzkin A, Abnet CC. Association between smoking and risk of bladder cancer among men and women. JAMA. United States; 2011;306:73745.

50. Brennan P, Bogillot O, Cordier S, Greiser E, Schill W, Vineis P, et al. Cigarette smoking and bladder cancer in men: a pooled analysis of 11 case-control studies. Int. J. Cancer. 2000;86:289-94.

51. Brennan P, Bogillot O, Greiser E, Chang-Claude J, Wahrendorf J, Cordier S, et al. The contribution of cigarette smoking to bladder cancer in women (pooled European data). Cancer Causes Control. 2001;12:411-7.

52. Pitard A, Brennan P, Clavel J, Greiser E, Lopez-Abente G, Chang-Claude J, et al. Cigar, pipe, and cigarette smoking and bladder cancer risk in European men. Cancer Causes Control. 2001;12:5516.

53. Cumberbatch MG, Rota M, Catto JWF, La Vecchia C. The Role of Tobacco Smoke in Bladder and Kidney Carcinogenesis: A Comparison of Exposures and Meta-analysis of Incidence and Mortality Risks. Eur. Urol. 2015;

54. Van Hemelrijck MJJ, Michaud DS, Connolly GN, Kabir Z. Secondhand smoking, 4-aminobiphenyl, and bladder cancer: two meta-analyses. Cancer Epidemiol. Biomarkers Prev. 2009;18:1312-20.

55. Lee PN, Hamling J. Systematic review of the relation between smokeless tobacco and cancer in Europe and North America. BMC Med. 2009;7:36.

56. Keimling M, Behrens G, Schmid D, Jochem C, Leitzmann MF. The association between physical activity and bladder cancer: systematic review and meta-analysis. Br. J. Cancer. 2014;110:1862-70.

57. Turati F, Pelucchi C, Galeone C, Decarli A, La Vecchia C. Personal hair dye use and bladder cancer: a meta-analysis. Ann. Epidemiol. 2014;24:151-9.

58. Kelsh MA, Alexander DD, Kalmes RM, Buffler PA. Personal use of hair dyes and risk of bladder cancer: a meta-analysis of epidemiologic data. Cancer Causes Control. 2008;19:549-58.

59. Huncharek M, Kupelnick B. Personal use of hair dyes and the risk of bladder cancer: results of a meta-analysis. Public Health Rep. United States; 2005;120:31-8.

60. Takkouche B, Etminan M, Montes-Martínez A. Personal use of hair dyes and risk of cancer: a metaanalysis. JAMA. 2005;293:2516-25.

61. Cumberbatch MGK, Cox A, Teare D, Catto JWF. Contemporary Occupational Carcinogen Exposure and Bladder Cancer. JAMA Oncol. 2015;1-9.

62. Reulen RC, Kellen E, Buntinx F, Brinkman M, Zeegers MP. A meta-analysis on the association between bladder cancer and occupation. Scand. J. Urol. Nephrol. Suppl. 2008;64-78.

63. Kogevinas M, 't Mannetje A, Cordier S, Ranft U, González CA, Vineis P, et al. Occupation and bladder cancer among men in Western Europe. Cancer Causes Control. 2003;14:907-14.

64. Mannetje A, Kogevinas M, Chang-Claude J, Cordier S, Gonzalez CA, Hours M, et al. Occupation and bladder cancer in European women. Cancer Causes Control. NETHERLANDS; 1999;10:209_ 17.

65. Clavel J, Cordier S, Boccon-Gibod L, Hemon D. Tobacco and bladder cancer in males: increased risk for inhalers and smokers of black tobacco. Int. J. Cancer. UNITED STATES; 1989;44:60510.

66. D’Avanzo B, Negri E, La Vecchia C, Gramenzi A, Bianchi C, Franceschi S, et al. Cigarette smoking and bladder cancer. Eur. J. Cancer. ENGLAND; 1990;26:714-8.

67. D’Avanzo B, La Vecchia C, Negri E, Decarli A, Benichou J. Attributable risks for bladder cancer in northern Italy. Ann. Epidemiol. UNITED STATES; 1995;5:427-31.

68. Iscovich J, Castelletto R, Esteve J, Munoz N, Colanzi R, Coronel A, et al. Tobacco smoking, occupational exposure and bladder cancer in Argentina. Int. J. Cancer. UNITED STATES; 1987;40:734-40. 
Chapter 5 I Modifiable risk factors for the prevention of bladder cancer

69. Momas I, Daures JP, Festy B, Bontoux J, Gremy F. Bladder cancer and black tobacco cigarette smoking. Some results from a French case-control study. Eur. J. Epidemiol. NETHERLANDS; 1994;10:599-604.

70. De Stefani E, Correa P, Fierro L, Fontham E, Chen V, Zavala D. Black tobacco, mate, and bladder cancer. A case-control study from Uruguay. Cancer. UNITED STATES; 1991;67:536-40.

71. Samanic C, Kogevinas M, Dosemeci M, Malats N, Real FX, Garcia-Closas M, et al. Smoking and bladder cancer in Spain: effects of tobacco type, timing, environmental tobacco smoke, and gender. Cancer Epidemiol. Biomarkers Prev. United States; 2006;15:1348-54.

72. Cole P, Monson RR, Haning H, Friedell GH. Smoking and cancer $f$ the lower urinary tract. N. Engl. J. Med. UNITED STATES; 1971;284:129-34.

73. Morrison AS, Buring JE, Verhoek WG, Aoki K, Leck I, Ohno Y, et al. An international study of smoking and bladder cancer. J. Urol. UNITED STATES; 1984;131:650-4.

74. Zeegers MPA, Goldbohm RA, van den Brandt PA. A prospective study on active and environmental tobacco smoking and bladder cancer risk (The Netherlands). Cancer Causes Control. Netherlands; 2002;13:83-90.

75. Lopez-Abente G, Gonzalez CA, Errezola M, Escolar A, Izarzugaza I, Nebot M, et al. Tobacco smoke inhalation pattern, tobacco type, and bladder cancer in Spain. Am. J. Epidemiol. UNITED STATES; 1991;134:830-9.

76. Burch JD, Rohan TE, Howe GR, Risch HA, Hill GB, Steele R, et al. Risk of bladder cancer by source and type of tobacco exposure: a case-control study. Int. J. Cancer. UNITED STATES; 1989;44:622-8.

77. Hartge P, Silverman D, Hoover R, Schairer C, Altman R, Austin D, et al. Changing cigarette habits and bladder cancer risk: a case-control study. J. Natl. Cancer Inst. UNITED STATES; 1987;78:1119-25.

78. Castelao JE, Yuan JM, Skipper PL, Tannenbaum SR, Gago-Dominguez M, Crowder JS, et al. Gender- and smoking-related bladder cancer risk. J. Natl. Cancer Inst. United States; 2001;93:53845.

79. Howe GR, Burch JD, Miller AB, Cook GM, Esteve J, Morrison B, et al. Tobacco use, occupation, coffee, various nutrients, and bladder cancer. J. Natl. Cancer Inst. UNITED STATES; 1980;64:70113.

80. Lockwood K. On the etiology of bladder tumors in Kobenhavn-Frederiksberg. An inquiry of 369 patients and 369 controls. Acta Pathol. Microbiol. Scand. Suppl. Not Available; 1961;51 Suppl $1: 1-166$.

81. Hein DW. Molecular genetics and function of NAT1 and NAT2: role in aromatic amine metabolism and carcinogenesis. Mutat. Res. Netherlands; 2002;506-507:65-77.

82. Centers for Disease Control and Prevention. How tobacco smoke causes disease: The biology and behavioral basis for smoking-attributable disease: A report of the surgeon general. Atlanta (GA); 2010.

83. Pelucchi C, Bosetti C, Negri E, Malvezzi M, La Vecchia C. Mechanisms of disease: The epidemiology of bladder cancer. Nat. Clin. Pract. Urol. 2006;3:327-40.

84. Brinkman M, Zeegers MP. Nutrition, total fluid and bladder cancer. Scand. J. Urol. Nephrol. Suppl. 2008;25-36.

85. Liu RH. Potential synergy of phytochemicals in cancer prevention: mechanism of action. J. Nutr. 2004;134:3479S - 3485S.

86. Talalay P, Fahey JW. Phytochemicals from cruciferous plants protect against cancer by modulating carcinogen metabolism. J. Nutr. 2001;131:3027S - 33S.

87. Kirk SF, Cade JE, Barrett JH, Conner M. Diet and lifestyle characteristics associated with dietary supplement use in women. Public Health Nutr. 1999;2:69-73.

88. Nutting CM, Huddart RA. Retinoids in the prevention of bladder cancer. Expert Rev. Anticancer Ther. 2001;1:541-5.

89. International Agency for Research on Cancer. IARC Handbook of Cancer Prevention Volume 2: 
Carotenoids. Lyon, France: International Agency for Research on Cancer; 1998.

90. Stahl W, Sies H. Antioxidant activity of carotenoids. Mol. Aspects Med. 2003;24:345-51.

91. Jiang Q. Natural forms of vitamin E: metabolism, antioxidant, and anti-inflammatory activities and their role in disease prevention and therapy. Free Radic. Biol. Med. 2014;72:76-90.

92. Balkwill F, Mantovani A. Inflammation and cancer: back to Virchow? Lancet (London, England). England; 2001;357:539-45.

93. Das S. Vitamin E in the genesis and prevention of cancer. A review. Acta Oncol. NORWAY; 1994;33:615-9.

94. Jackson MI, Combs GF. Selenium and anticarcinogenesis: underlying mechanisms. Curr. Opin. Clin. Nutr. Metab. Care. 2008;11:718-26.

95. Mosby TT, Cosgrove M, Sarkardei S, Platt KL, Kaina B. Nutrition in adult and childhood cancer: role of carcinogens and anti-carcinogens. Anticancer Res. 2012;32:4171-92.

96. Micha R, Wallace SK, Mozaffarian D. Red and processed meat consumption and risk of incident coronary heart disease, stroke, and diabetes mellitus: a systematic review and meta-analysis. Circulation. 2010;121:2271-83.

97. Baena Ruiz R, Salinas Hernández P. Diet and cancer: risk factors and epidemiological evidence. Maturitas. 2014;77:202-8.

98. Choi SW, Mason JB. Folate and carcinogenesis: an integrated scheme. J. Nutr. 2000;130:129-32.

99. Duthie SJ, Narayanan S, Brand GM, Pirie L, Grant G. Impact of folate deficiency on DNA stability. J. Nutr. United States; 2002;132:2444S - 2449S.

100. Ulrich CM, Potter JD. Folate and Cancer-Timing Is Everything. JAMA. 2007;297:2408.

101. Lee JE, Li H, Chan AT, Hollis BW, Lee I-M, Stampfer MJ, et al. Circulating levels of vitamin $\mathrm{D}$ and colon and rectal cancer: the Physicians' Health Study and a meta-analysis of prospective studies. Cancer Prev. Res. (Phila). United States; 2011;4:735-43.

102. Kim Y, Je Y. Vitamin D intake, blood 25(OH)D levels, and breast cancer risk or mortality: a metaanalysis. Br. J. Cancer. 2014;110:2772-84.

103. Feldman D, Krishnan A V, Swami S, Giovannucci E, Feldman BJ. The role of vitamin D in reducing cancer risk and progression. Nat Rev Cancer. 2014;14:342-57.

104. Silberstein JL, Parsons JK. Evidence-based principles of bladder cancer and diet. Urology. 2010;75:340-6.

105. Villanueva CM, Fernández F, Malats N, Grimalt JO, Kogevinas M. Meta-analysis of studies on individual consumption of chlorinated drinking water and bladder cancer. J. Epidemiol. Community Health. 2003;57:166-73.

106. Costet N, Villanueva CM, Jaakkola JJK, Kogevinas M, Cantor KP, King WD, et al. Water disinfection by-products and bladder cancer: is there a European specificity? A pooled and metaanalysis of European case-control studies. Occup. Environ. Med. 2011;68:379-85.

107. Tsuji JS, Alexander DD, Perez V, Mink PJ. Arsenic exposure and bladder cancer: quantitative assessment of studies in human populations to detect risks at low doses. Toxicology. 2014;317:1730.

108. Chu H-A, Crawford-Brown DJ. Inorganic arsenic in drinking water and bladder cancer: a metaanalysis for dose-response assessment. Int. J. Environ. Res. Public Health. 2006;3:316-22.

109. Rogers CJ, Colbert LH, Greiner JW, Perkins SN, Hursting SD. Physical activity and cancer prevention : pathways and targets for intervention. Sports Med. 2008;38:271-96.

110. Letašiová S, Medve’ová A, Šovčíková A, Dušinská M, Volkovová K, Mosoiu C, et al. Bladder cancer, a review of the environmental risk factors. Environ. Health. 2012;11 Suppl 1:S11.

111. Alavanja MCR, Bonner MR. Occupational pesticide exposures and cancer risk: a review. J. Toxicol. Environ. Health. B. Crit. Rev. 2012;15:238-63.

112. Ntzani E, Chondrogiorgi M, Ntritsos G, Evangelou E, Tzoulaki I. Literature re view on epidemiological studies linking exposure to pesticides and health effect. EFSA supporting publication 2013:EN-497. 2013.

113. IAC Monographs on the Evaluation of Carcinogenic Risks to Humans, Volume 53, Occupational 
Chapter 5 I Modifiable risk factors for the prevention of bladder cancer

Exposures in Insecticide Application, and Some Pesticides. Lyon, France; 1991.

114. Tramèr MR, Reynolds DJ, Moore RA, McQuay HJ. Impact of covert duplicate publication on meta-analysis: a case study. BMJ. 1997;315:635-40.

115. Berrigan D, Dodd K, Troiano RP, Krebs-Smith SM, Barbash RB. Patterns of health behavior in U.S. adults. Prev. Med. (Baltim). 2003;36:615-23.

116. Lim SS, Vos T, Flaxman AD, Danaei G, Shibuya K, Adair-Rohani H, et al. A comparative risk assessment of burden of disease and injury attributable to 67 risk factors and risk factor clusters in 21 regions, 1990-2010: a systematic analysis for the Global Burden of Disease Study 2010. Lancet. 2012;380:2224-60.

117. Stein CJ, Colditz GA. Modifiable risk factors for cancer. Br. J. Cancer. 2004;90:299-303.

118. Yusuf S, Hawken S, Ounpuu S, Dans T, Avezum A, Lanas F, et al. Effect of potentially modifiable risk factors associated with myocardial infarction in 52 countries (the INTERHEART study): casecontrol study. Lancet. 364:937-52.

119. Danaei G, Ding EL, Mozaffarian D, Taylor B, Rehm J, Murray CJL, et al. The preventable causes of death in the United States: comparative risk assessment of dietary, lifestyle, and metabolic risk factors. PLoS Med. 2009;6:e1000058.

120. Riboli E, Norat T. Epidemiologic evidence of the protective effect of fruit and vegetables on cancer risk. Am. J. Clin. Nutr. 2003;78:559S - 569S.

121. Liu B, Mao Q, Lin Y, Zhou F, Xie L. The association of cruciferous vegetables intake and risk of bladder cancer: a meta-analysis. World J. Urol. 2013;31:127-33.

122. Vieira AR, Vingeliene S, Chan DSM, Aune D, Abar L, Navarro Rosenblatt D, et al. Fruits, vegetables, and bladder cancer risk: a systematic review and meta-analysis. Cancer Med. 2015;4:136-46.

123. Liu H, Wang X-C, Hu G-H, Guo Z-F, Lai P, Xu L, et al. Fruit and vegetable consumption and risk of bladder cancer. Eur. J. Cancer Prev. 2015;24:508-16.

124. Xu C, Zeng X-T, Liu T-Z, Zhang C, Yang Z-H, Li S, et al. Fruits and vegetables intake and risk of bladder cancer: a PRISMA-compliant systematic review and dose-response meta-analysis of prospective cohort studies. Medicine (Baltimore). 2015;94:e759.

125. Zeegers MP, Tan FE, Goldbohm RA, van den Brandt PA. Are coffee and tea consumption associated with urinary tract cancer risk? A systematic review and meta-analysis. Int. J. Epidemiol. 2001;30:353-62.

126. Zhang Y-F, Xu Q, Lu J, Wang P, Zhang H-W, Zhou L, et al. Tea consumption and the incidence of cancer: a systematic review and meta-analysis of prospective observational studies. Eur. J. Cancer Prev. 2015;24:353-62.

127. Wu S, Li F, Huang X, Hua Q, Huang T, Liu Z, et al. The association of tea consumption with bladder cancer risk: a meta-analysis. Asia Pac. J. Clin. Nutr. 2013;22:128-37.

128. Wang X, Lin Y-W, Wang S, Wu J, Mao Q-Q, Zheng X-Y, et al. A meta-analysis of tea consumption and the risk of bladder cancer. Urol. Int. 2013;90:10-6.

129. Boehm K, Borrelli F, Ernst E, Habacher G, Hung SK, Milazzo S, et al. Green tea (Camellia sinensis) for the prevention of cancer. Cochrane database Syst. Rev. 2009;CD005004.

130. Qin J, Xie B, Mao Q, Kong D, Lin Y, Zheng X. Tea consumption and risk of bladder cancer: a meta-analysis. World J. Surg. Oncol. 2012;10:172.

131. Arab L. Epidemiologic evidence on coffee and cancer. Nutr. Cancer. 2010;62:271-83.

132. Pelucchi C, La Vecchia C. Alcohol, coffee, and bladder cancer risk: a review of epidemiological studies. Eur. J. Cancer Prev. 2009;18:62-8.

133. Zhang Z-H, Yang L-S, Sun Y-H. The association between milk consumption and bladder cancer risk: appraisal of a recent meta-analysis. Nutr. Cancer. 2012;64:1288-9.

134. Lampe JW. Dairy products and cancer. J. Am. Coll. Nutr. 2011;30:464S - 70S.

135. Li F, An S, Zhou Y, Liang Z, Jiao Z, Jing Y, et al. Milk and dairy consumption and risk of bladder cancer: a meta-analysis. Urology. 2011;78:1298-305.

136. Wang C, Jiang H. Meat intake and risk of bladder cancer: a meta-analysis. Med. Oncol. 2012;29:848-55. 
137. Mao Q, Lin Y, Zheng X, Qin J, Yang K, Xie L. A meta-analysis of alcohol intake and risk of bladder cancer. Cancer Causes Control. 2010;21:1843-50.

138. de Menezes RF, Bergmann A, Thuler LCS. Alcohol consumption and risk of cancer: a systematic literature review. Asian Pac. J. Cancer Prev. 2013;14:4965-72.

139. Bagnardi V, Blangiardo M, La Vecchia C, Corrao G. A meta-analysis of alcohol drinking and cancer risk. Br. J. Cancer. 2001;85:1700-5.

140. Zeegers MP, Tan FE, Verhagen AP, Weijenberg MP, van den Brandt PA. Elevated risk of cancer of the urinary tract for alcohol drinkers: a meta-analysis. Cancer Causes Control. 1999;10:445-51.

141. Fang D, Tan F, Wang C, Zhu X, Xie L. Egg intake and bladder cancer risk: A meta-analysis. Exp. Ther. Med. 2012;4:906-12.

142. Musa-Veloso K, Card JW, Wong AW, Cooper DA. Influence of observational study design on the interpretation of cancer risk reduction by carotenoids. Nutr. Rev. 2009;67:527-45.

143. Coulter ID, Hardy ML, Morton SC, Hilton LG, Tu W, Valentine D, et al. Antioxidants vitamin $\mathrm{C}$ and vitamin e for the prevention and treatment of cancer. J. Gen. Intern. Med. United States; 2006;21:735-44.

144. MacLean CH, Newberry SJ, Mojica WA, Khanna P, Issa AM, Suttorp MJ, et al. Effects of omega-3 fatty acids on cancer risk: a systematic review. JAMA. 2006;295:403-15.

145. Mao S, Huang S. Zinc and copper levels in bladder cancer: a systematic review and meta-analysis. Biol. Trace Elem. Res. 2013;153:5-10.

146. Psaltopoulou T, Kosti RI, Haidopoulos D, Dimopoulos M, Panagiotakos DB. Olive oil intake is inversely related to cancer prevalence: a systematic review and a meta-analysis of 13,800 patients and 23,340 controls in 19 observational studies. Lipids Health Dis. England; 2011;10:127.

147. Lotan Y, Daudon M, Bruyère F, Talaska G, Strippoli G, Johnson RJ, et al. Impact of fluid intake in the prevention of urinary system diseases: a brief review. Curr. Opin. Nephrol. Hypertens. 2013;22 Suppl 1:S1-10.

148. Stelmach R, Clasen T. Household Water Quantity and Health: A Systematic Review. Int. J. Environ. Res. Public Health. 2015;12:5954-74.

149. Zeegers MP, Tan FE, Dorant E, van Den Brandt PA. The impact of characteristics of cigarette smoking on urinary tract cancer risk: a meta-analysis of epidemiologic studies. Cancer. 2000;89:630-9.

150. Crivelli JJ, Xylinas E, Kluth LA, Rieken M, Rink M, Shariat SF. Effect of smoking on outcomes of urothelial carcinoma: a systematic review of the literature. Eur. Urol. 2014;65:742-54.

151. Sasco AJ, Secretan MB, Straif K. Tobacco smoking and cancer: a brief review of recent epidemiological evidence. Lung Cancer. 2004;45 Suppl 2:S3-9.

152. Vineis P, Alavanja M, Garte S. Dose-response relationship in tobacco-related cancers of bladder and lung: a biochemical interpretation. Int. J. Cancer. 2004;108:2-7.

153. Shiels MS, Gibson T, Sampson J, Albanes D, Andreotti G, Beane Freeman L, et al. Cigarette smoking prior to first cancer and risk of second smoking-associated cancers among survivors of bladder, kidney, head and neck, and stage I lung cancers. J. Clin. Oncol. United States; 2014;32:3989-95.

154. 't Mannetje A, Kogevinas M, Chang-Claude J, Cordier S, González CA, Hours M, et al. Smoking as a confounder in case-control studies of occupational bladder cancer in women. Am. J. Ind. Med. 1999;36:75-82.

155. Akl EA, Gaddam S, Gunukula SK, Honeine R, Jaoude PA, Irani J. The effects of waterpipe tobacco smoking on health outcomes: a systematic review. Int. J. Epidemiol. 2010;39:834-57.

156. Rollison DE, Helzlsouer KJ, Pinney SM. Personal hair dye use and cancer: a systematic literature review and evaluation of exposure assessment in studies published since 1992. J. Toxicol. Environ. Health. B. Crit. Rev. 9:413-39.

157. Bosetti C, Pira E, La Vecchia C. Bladder cancer risk in painters: a review of the epidemiological evidence, 1989-2004. Cancer Causes Control. 2005;16:997-1008.

158. Guha N, Steenland NK, Merletti F, Altieri A, Cogliano V, Straif K. Bladder cancer risk in painters: a meta-analysis. Occup. Environ. Med. 2010;67:568-73.

159. Bachand A, Mundt KA, Mundt DJ, Carlton LE. Meta-analyses of occupational exposure as 
Chapter 5 I Modifiable risk factors for the prevention of bladder cancer

a painter and lung and bladder cancer morbidity and mortality 1950-2008. Crit. Rev. Toxicol. 2010;40:101-25.

160. Takkouche B, Regueira-Méndez C, Montes-Martínez A. Risk of cancer among hairdressers and related workers: a meta-analysis. Int. J. Epidemiol. 2009;38:1512-31.

161. Harling M, Schablon A, Schedlbauer G, Dulon M, Nienhaus A. Bladder cancer among hairdressers: a meta-analysis. Occup. Environ. Med. England; 2010;67:351-8.

162. 't Mannetje A, Pearce N. Bladder cancer risk in sales workers: artefact or cause for concern? Am. J. Ind. Med. 2006;49:175-86.

163. Boffetta P, Silverman DT. A meta-analysis of bladder cancer and diesel exhaust exposure. Epidemiology. 2001;12:125-30.

164. Manju L, George PS, Mathew A. Urinary bladder cancer risk among motor vehicle drivers: a metaanalysis of the evidence, 1977-2008. Asian Pac. J. Cancer Prev. 2009;10:287-94.

165. Acquavella J, Olsen G, Cole P, Ireland B, Kaneene J, Schuman S, et al. Cancer among farmers: a meta-analysis. Ann. Epidemiol. 1998;8:64-74.

166. Gaertner RRW, Thériault GP. Risk of bladder cancer in foundry workers: a meta-analysis. Occup. Environ. Med. 2002;59:655-63.

167. Mastrangelo G, Fedeli U, Fadda E, Milan G, Lange JH. Epidemiologic evidence of cancer risk in textile industry workers: a review and update. Toxicol. Ind. Health. 2002;18:171-81.

168. Tokumaru O, Haruki K, Bacal K, Katagiri T, Yamamoto T, Sakurai Y. Incidence of cancer among female flight attendants: a meta-analysis. J. Travel Med. 13:127-32.

169. Buja A, Mastrangelo G, Perissinotto E, Grigoletto F, Frigo AC, Rausa G, et al. Cancer Incidence among Female Flight Attendants: A Meta-Analysis of Published Data. J. Women's Heal. 2006;15:98-105.

170. Wong O, Raabe GK. A critical review of cancer epidemiology in the petroleum industry, with a meta-analysis of a combined database of more than 350,000 workers. Regul. Toxicol. Pharmacol. 2000;32:78-98.

171. Baena AV, Allam MF, Díaz-Molina C, Del Castillo AS, Abdel-Rahman AG, Navajas RF-C. Urinary bladder cancer and the petroleum industry: a quantitative review. Eur. J. Cancer Prev. 2006;15:493-7.

172. Greenberg RS, Mandel JS, Pastides H, Britton NL, Rudenko L, Starr TB. A meta-analysis of cohort studies describing mortality and cancer incidence among chemical workers in the United States and western Europe. Epidemiology. 2001;12:727-40.

173. Vlaanderen J, Straif K, Ruder A, Blair A, Hansen J, Lynge E, et al. Tetrachloroethylene Exposure and Bladder Cancer Risk: A Meta-Analysis of Dry-Cleaning-Worker Studies. Environ. Health Perspect. 2014;122:661-6.

174. Fu H, Boffetta P. Cancer and occupational exposure to inorganic lead compounds: a meta-analysis of published data. Occup. Environ. Med. 1995;52:73-81.

175. Cole P, Mandel JS, Collins JJ. Acrylonitrile and cancer: a review of the epidemiology. Regul. Toxicol. Pharmacol. United States; 2008;52:342-51.

176. Collins JJ, Acquavella JF. Review and meta-analysis of studies of acrylonitrile workers. Scand. J. Work. Environ. Health. 1998;24 Suppl 2:71-80.

177. Bosetti C, Boffetta P, La Vecchia C. Occupational exposures to polycyclic aromatic hydrocarbons, and respiratory and urinary tract cancers: a quantitative review to 2005. Ann. Oncol. 2007;18:43146.

178. Boffetta P, Jourenkova N, Gustavsson P. Cancer risk from occupational and environmental exposure to polycyclic aromatic hydrocarbons. Cancer Causes Control. 1997;8:444-72.

179. Rota M, Bosetti C, Boccia S, Boffetta P, La Vecchia C. Occupational exposures to polycyclic aromatic hydrocarbons and respiratory and urinary tract cancers: an updated systematic review and a meta-analysis to 2014. Arch. Toxicol. 2014;88:1479-90.

180. Calvert GM, Ward E, Schnorr TM, Fine LJ. Cancer risks among workers exposed to metalworking fluids: a systematic review. Am. J. Ind. Med. 1998;33:282-92. 
181. Mundt KA, Birk T, Burch MT. Critical review of the epidemiological literature on occupational exposure to perchloroethylene and cancer. Int. Arch. Occup. Environ. Health. 2003;76:473-91.

182. Goodman M, Morgan RW, Ray R, Malloy CD, Zhao K. Cancer in asbestos-exposed occupational cohorts: a meta-analysis. Cancer Causes Control. 1999;10:453-65.

183. International Labour Office (ILO). International Standard Classification of Occupations. Revised Edition 1968. Geneva: International Labour Office (ILO); 1969.

184. Statistical Office of the United Nations. International standard industrial classification of all economic activities. Statistical papers series M, number 4, revision 2. 1968. 


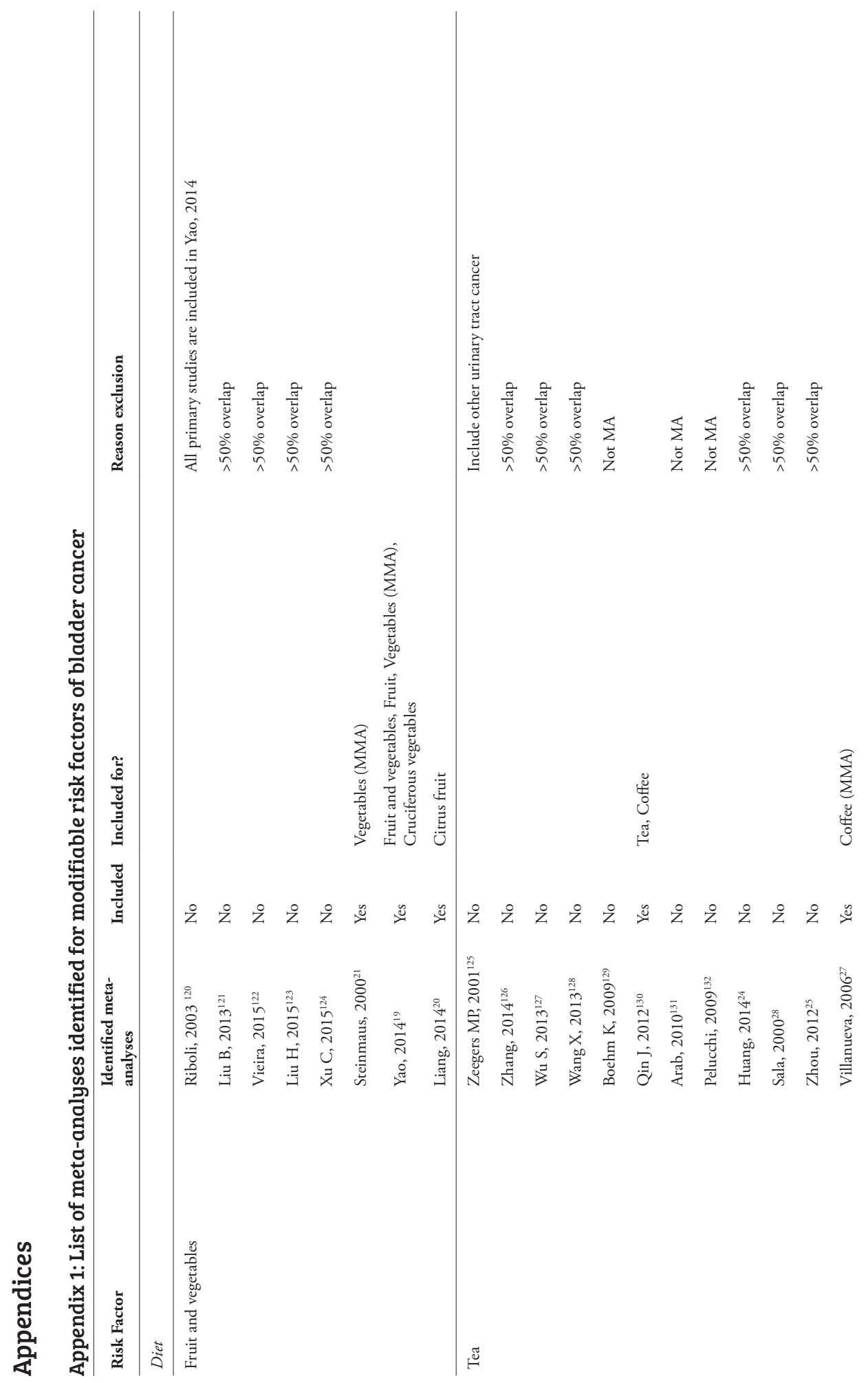




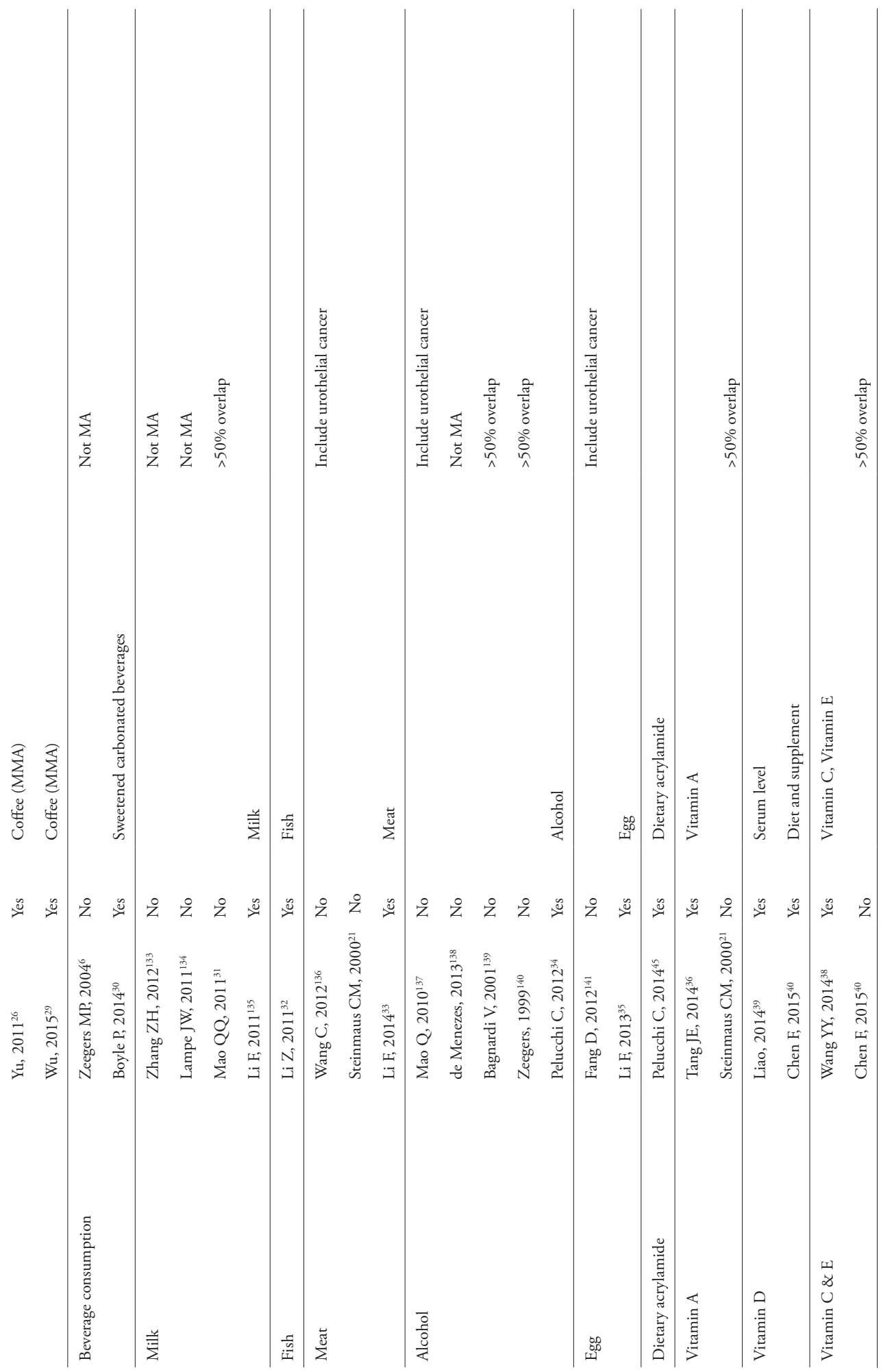




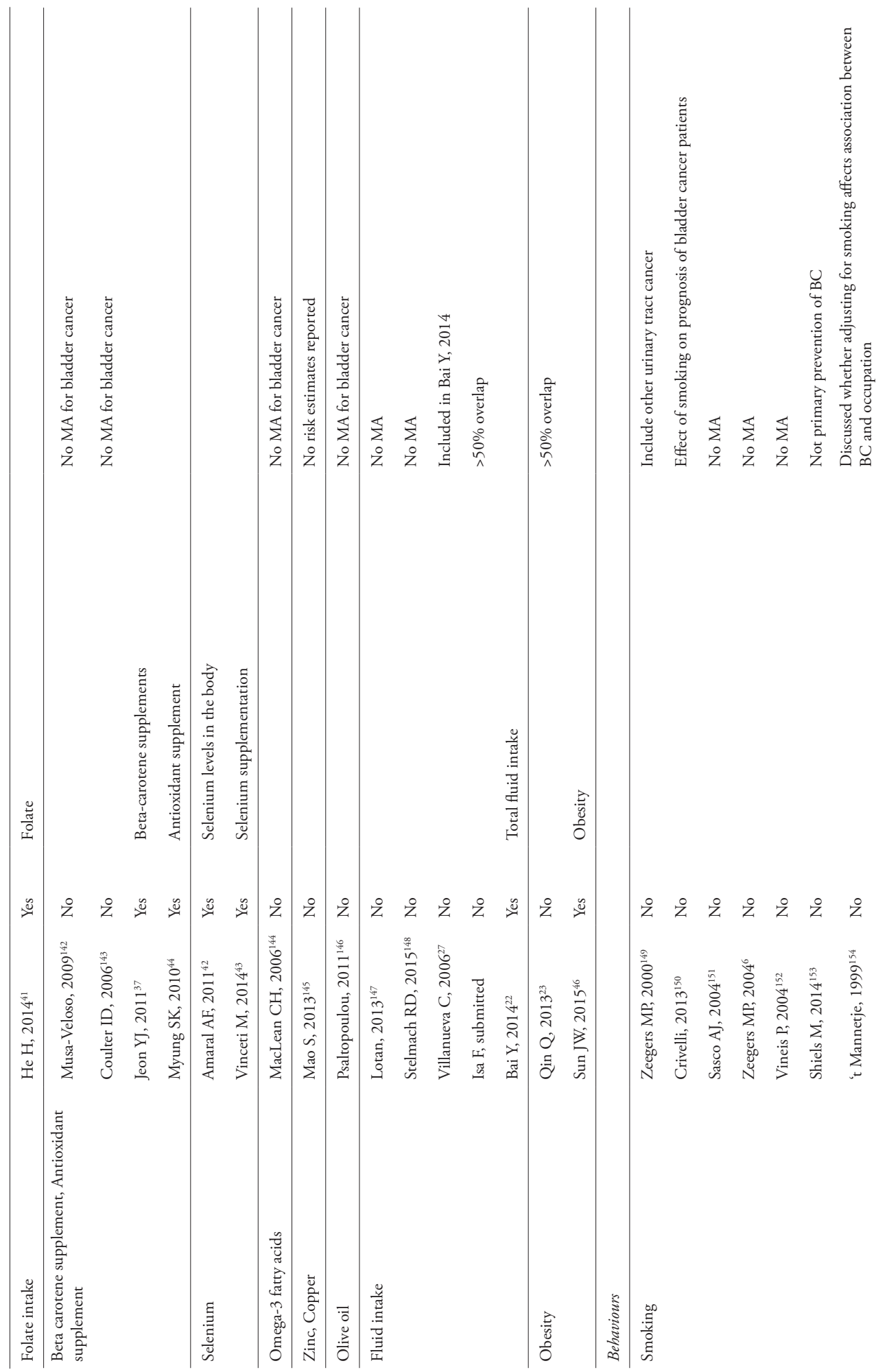



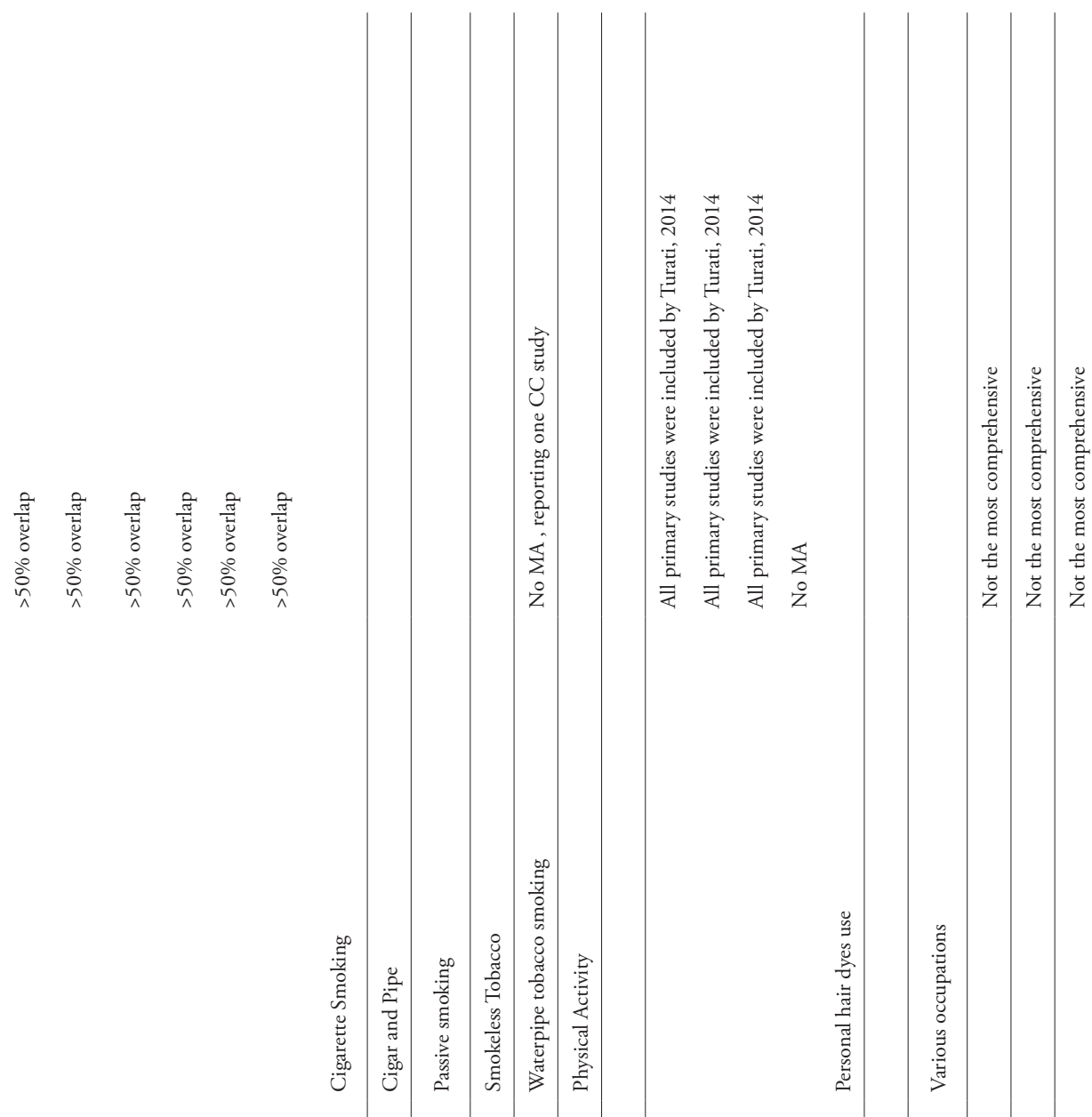

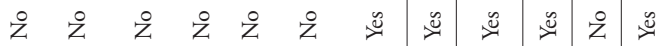

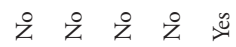

乐

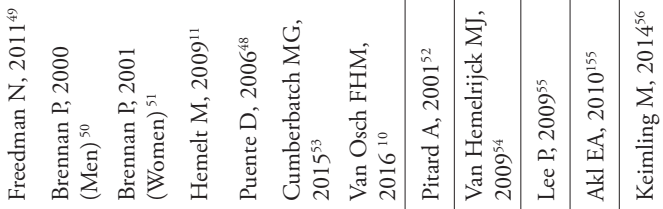

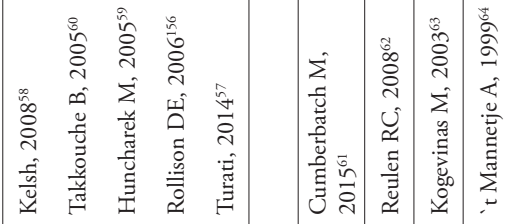
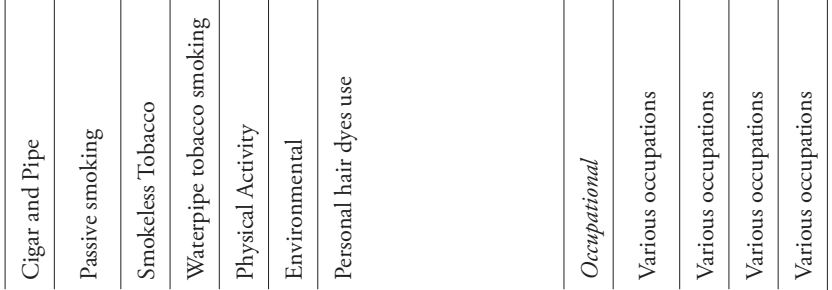


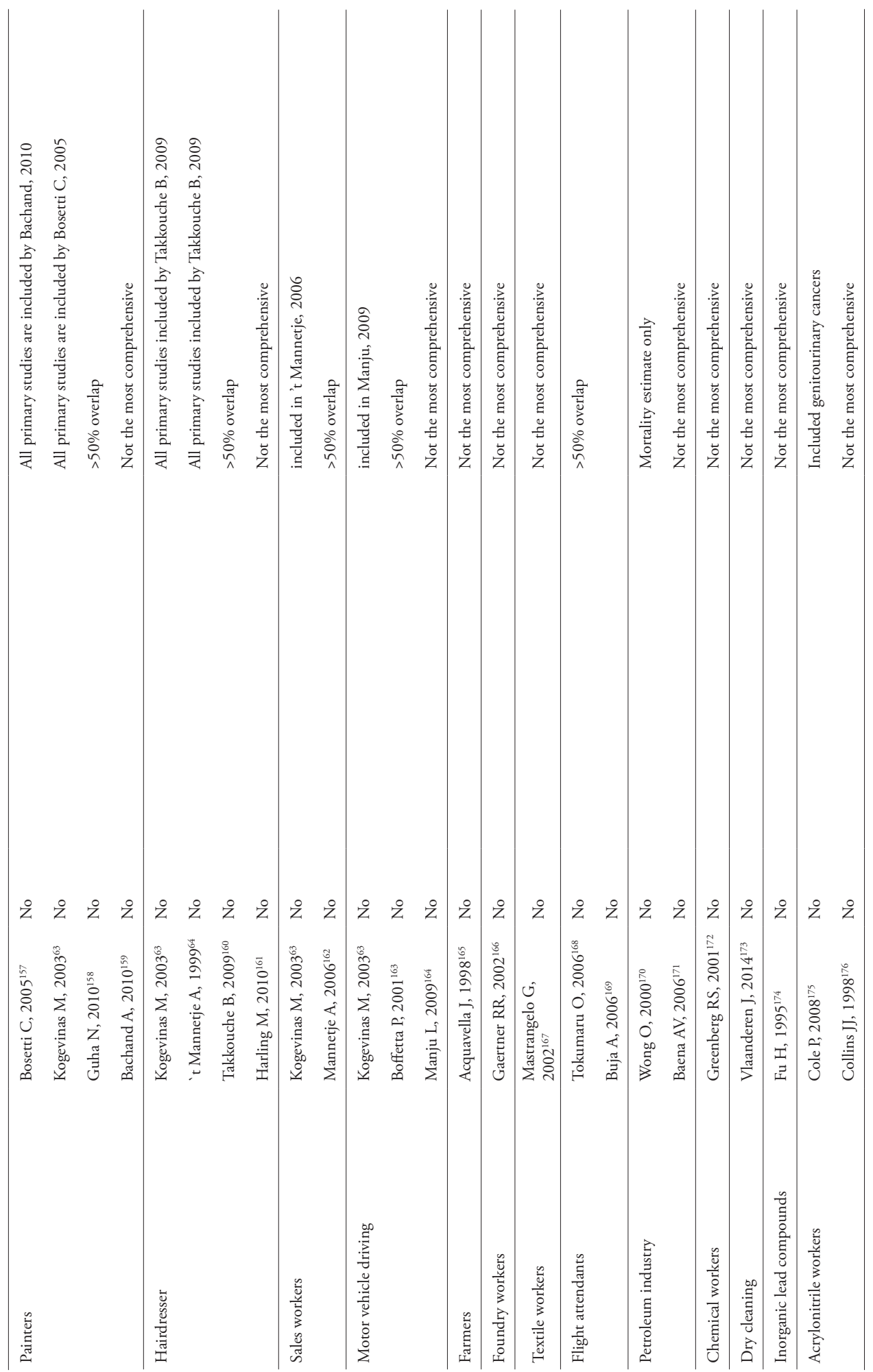


Chapter $\mathbf{5}$ I Modifiable risk factors for the prevention of bladder cancer

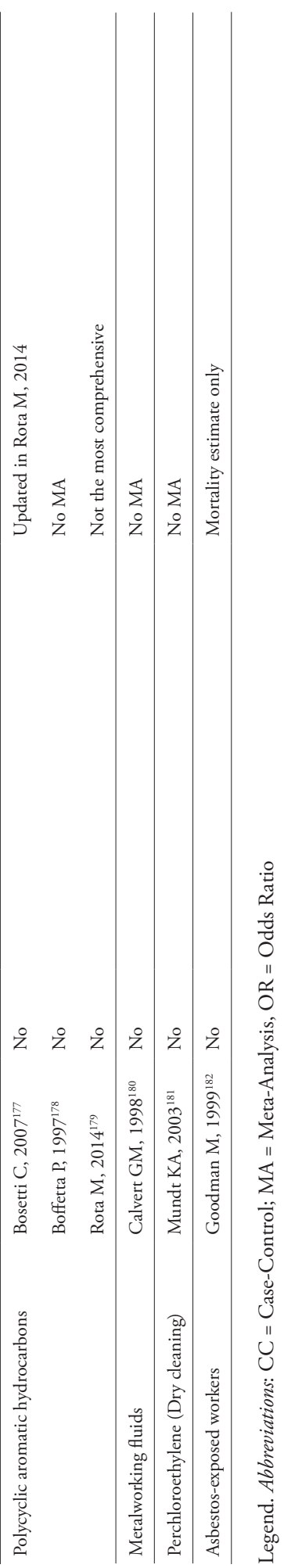


Chapter 5 I Modifiable risk factors for the prevention of bladder cancer

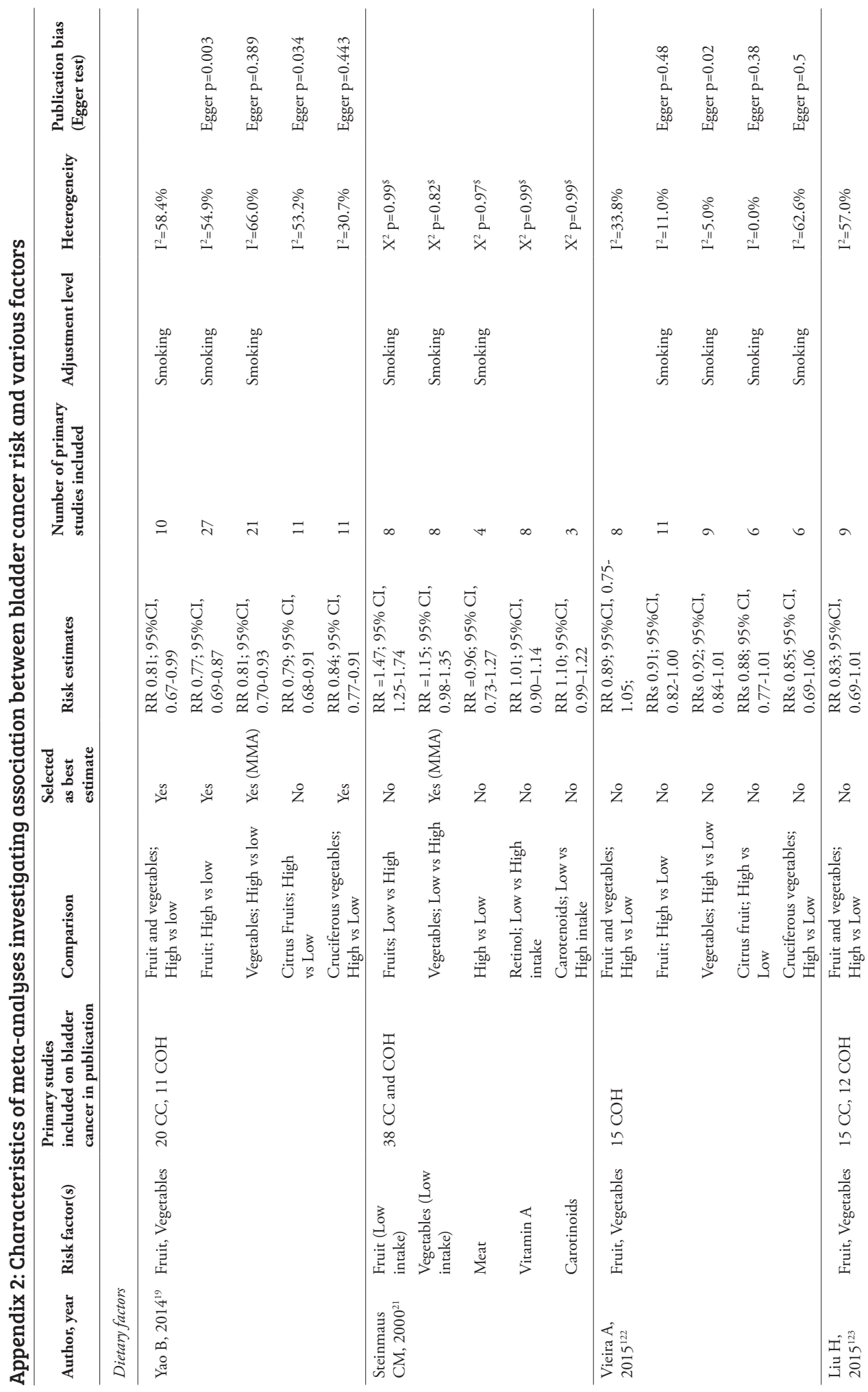


Chapter $\mathbf{5}$ | Modifiable risk factors for the prevention of bladder cancer

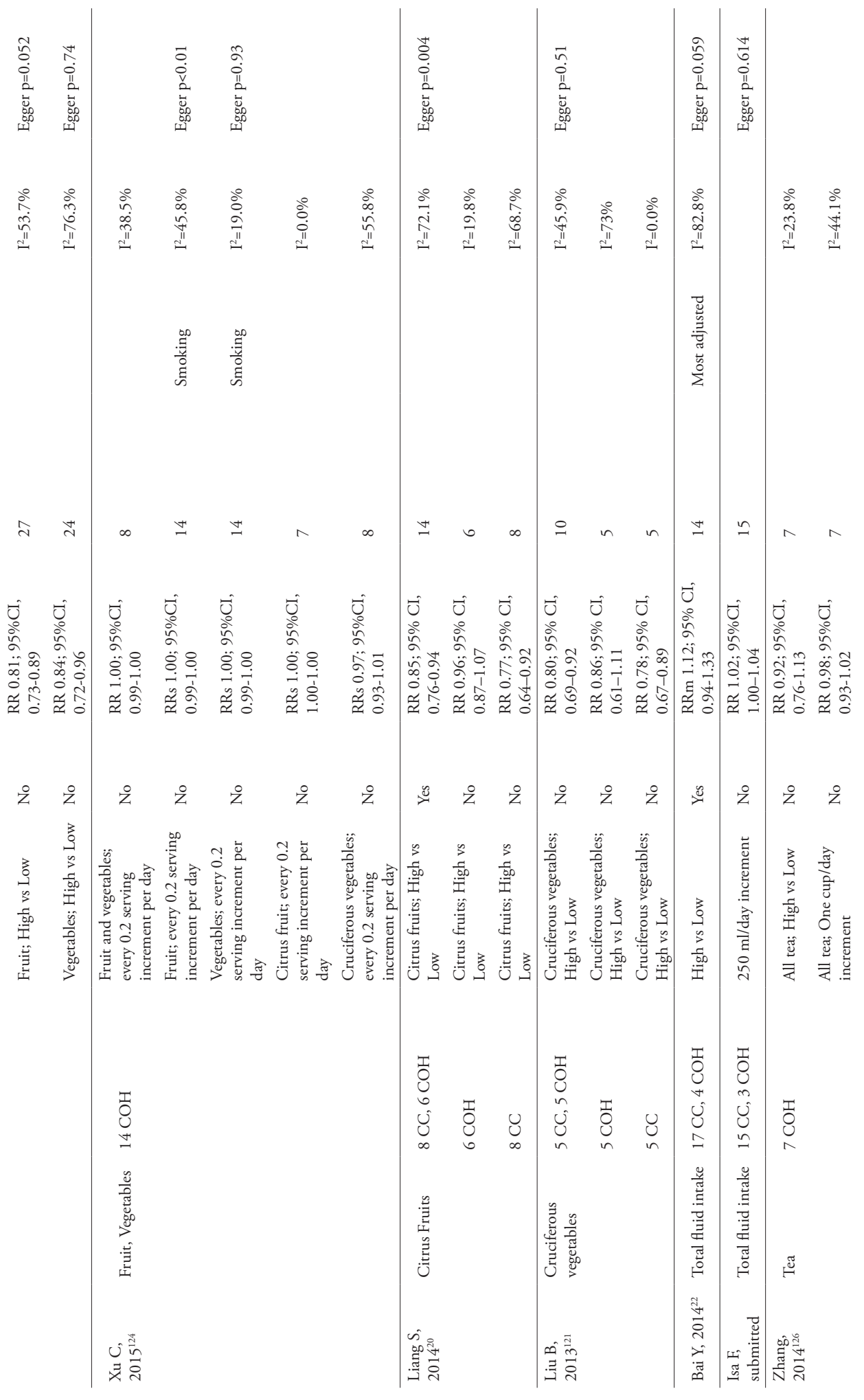


Chapter 5 I Modifiable risk factors for the prevention of bladder cancer

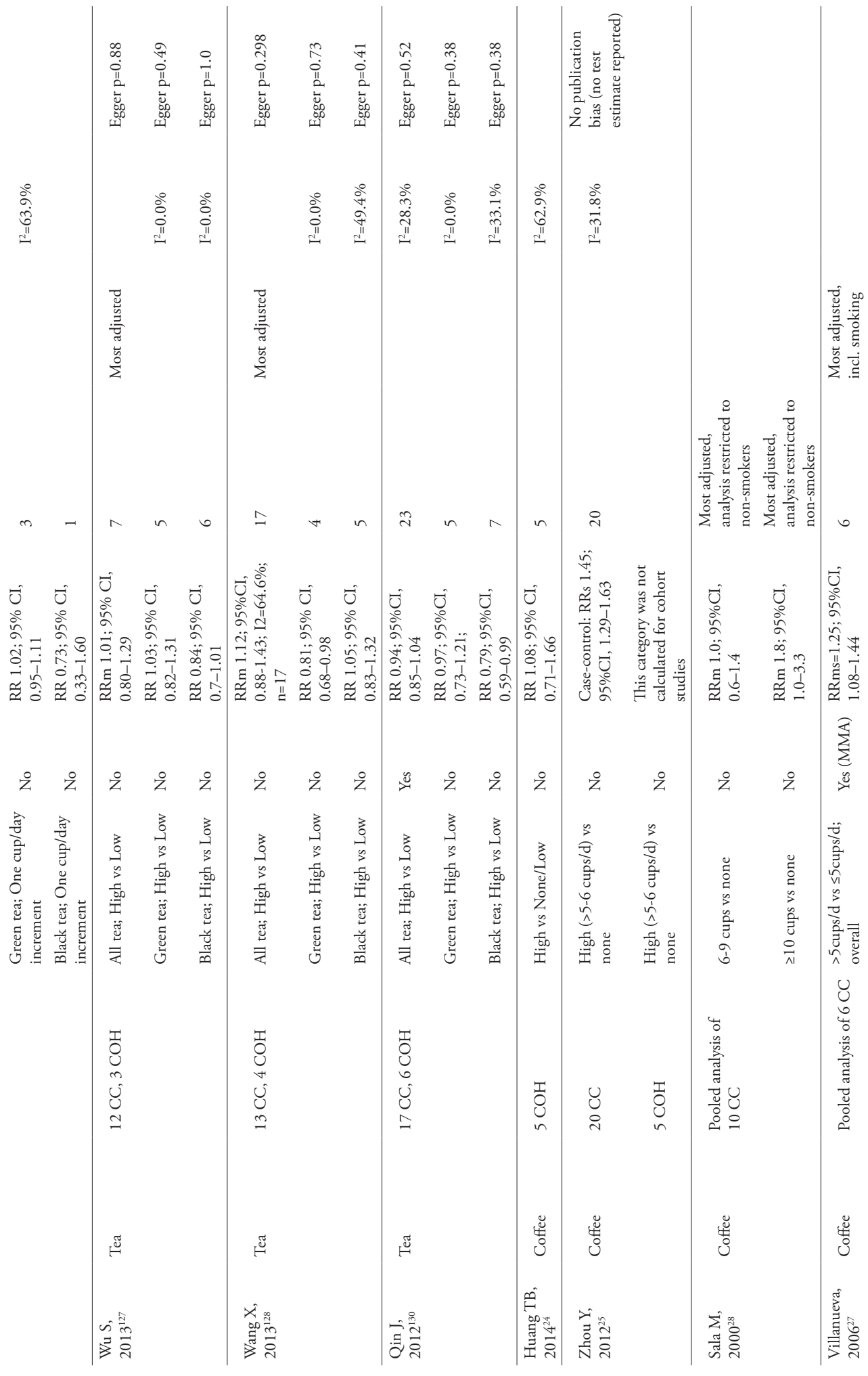


Chapter $\mathbf{5}$ I Modifiable risk factors for the prevention of bladder cancer

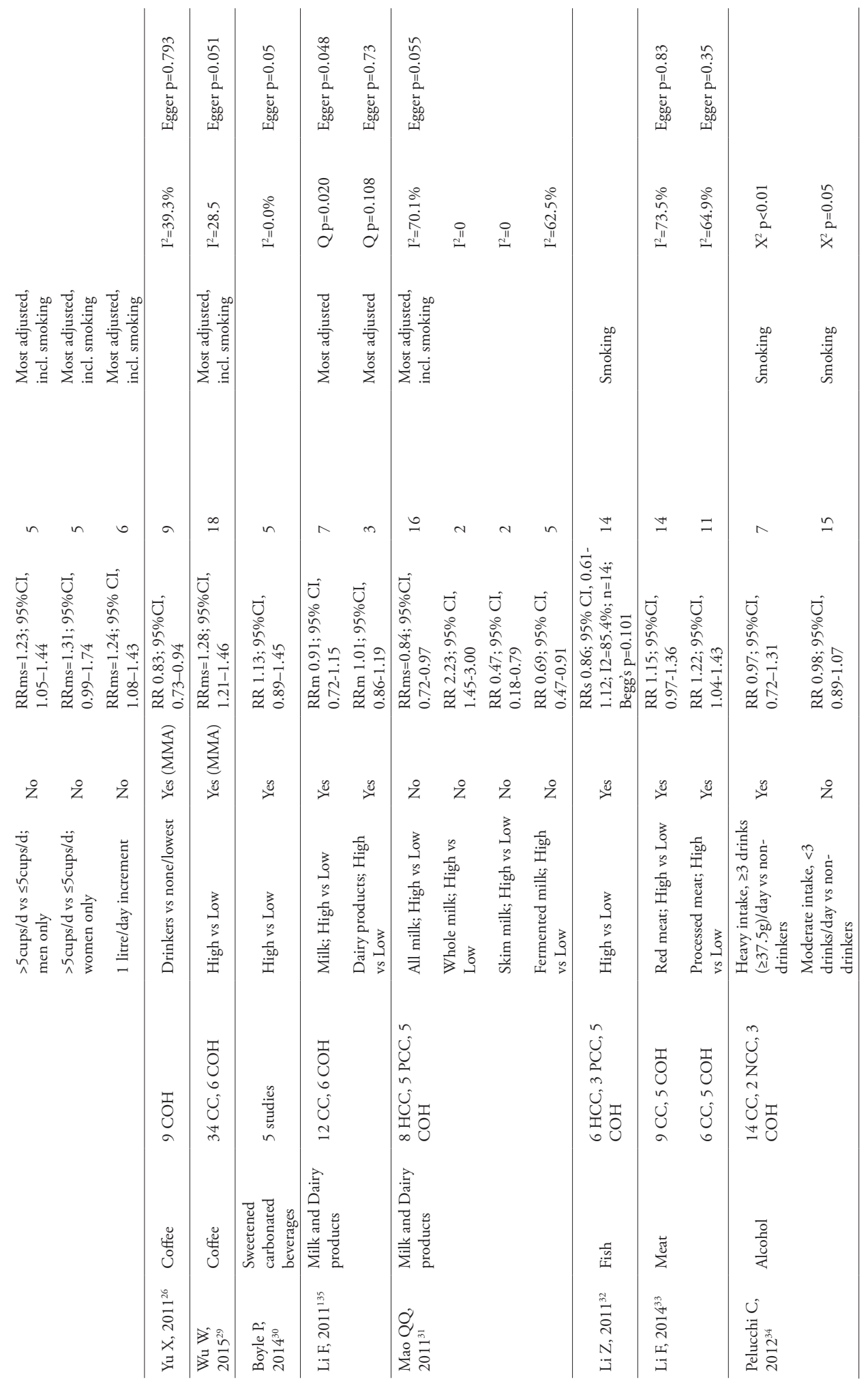


Chapter 5 I Modifiable risk factors for the prevention of bladder cancer

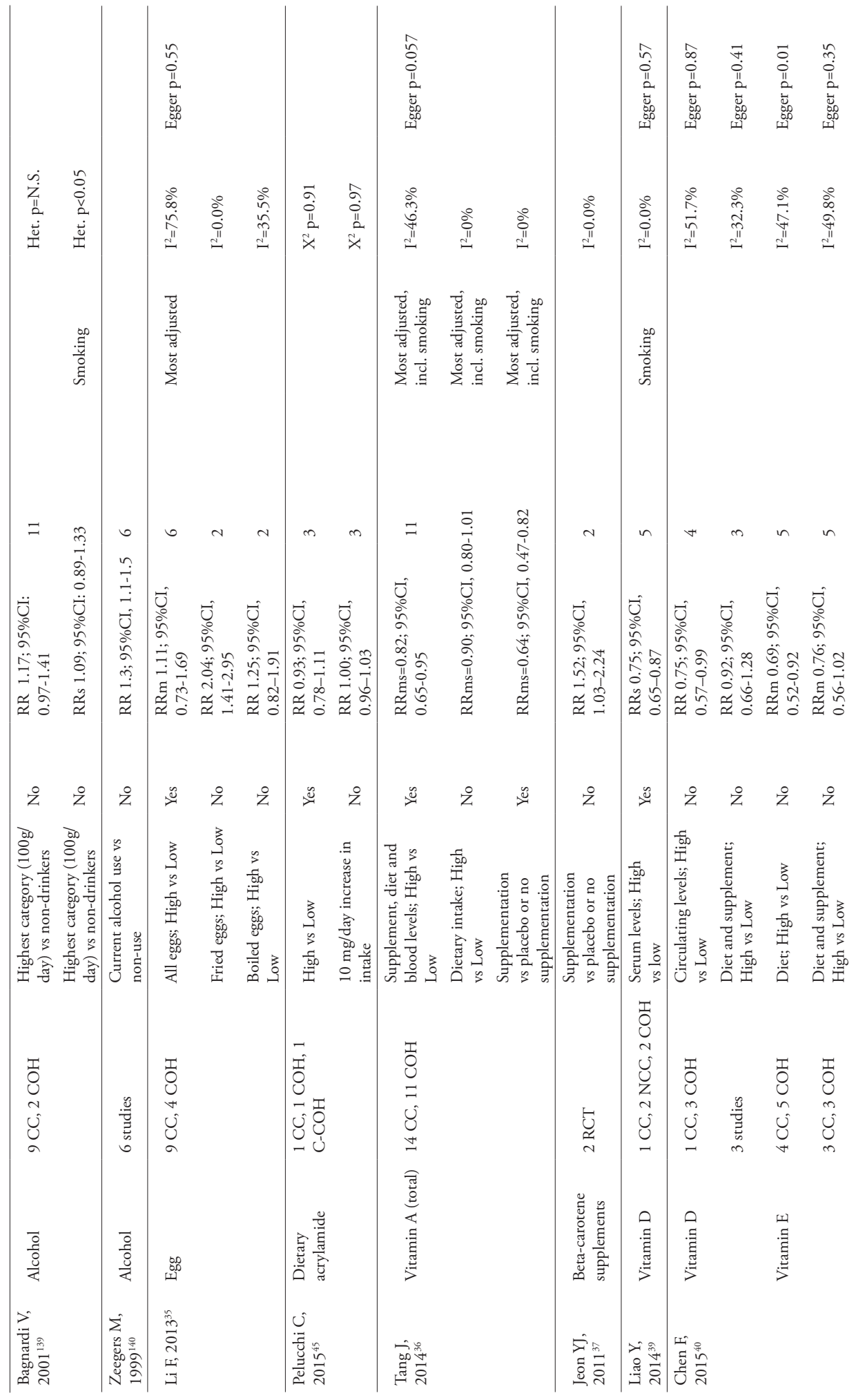


Chapter $\mathbf{5}$ | Modifiable risk factors for the prevention of bladder cancer

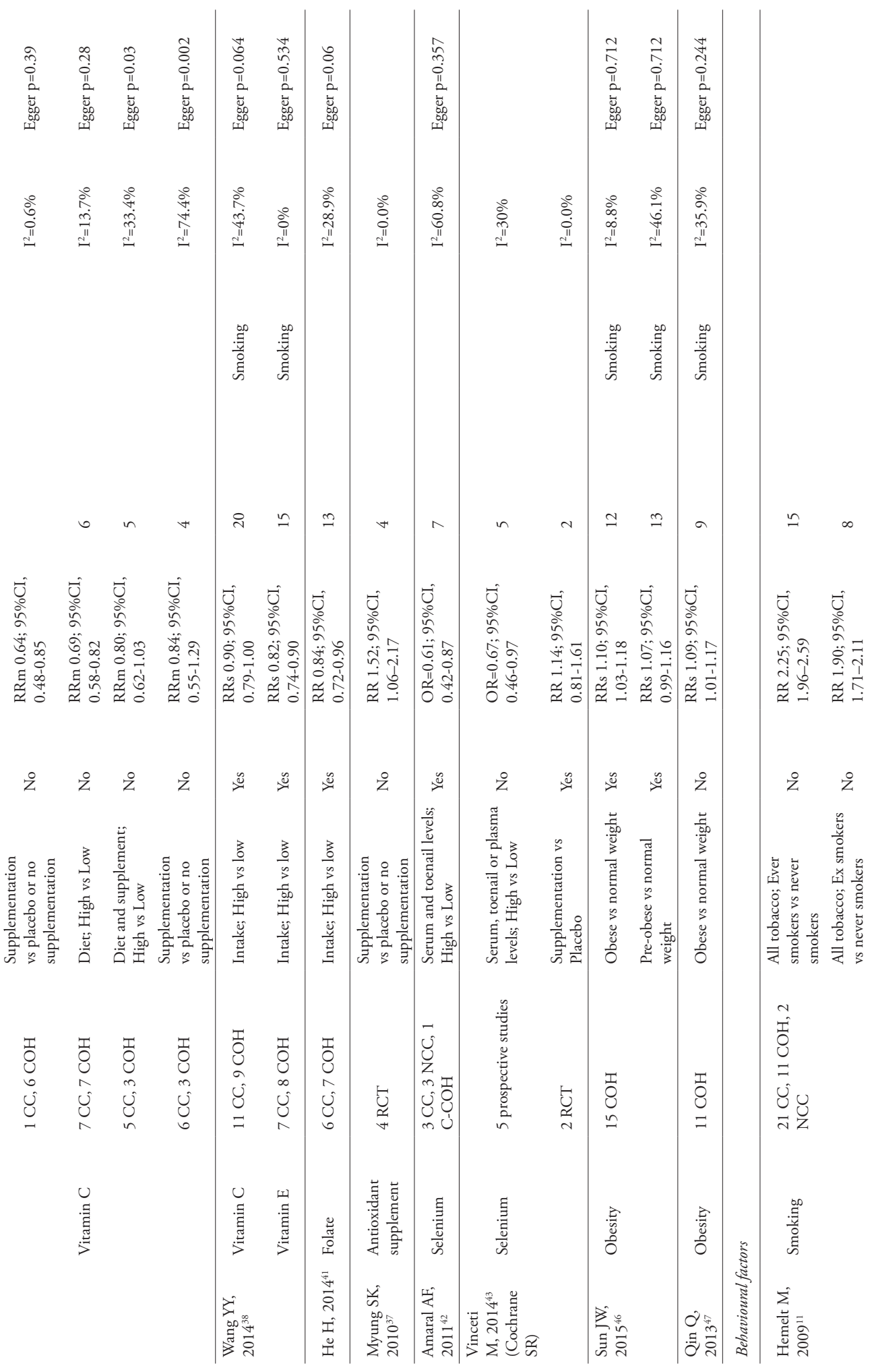


Chapter 5 I Modifiable risk factors for the prevention of bladder cancer

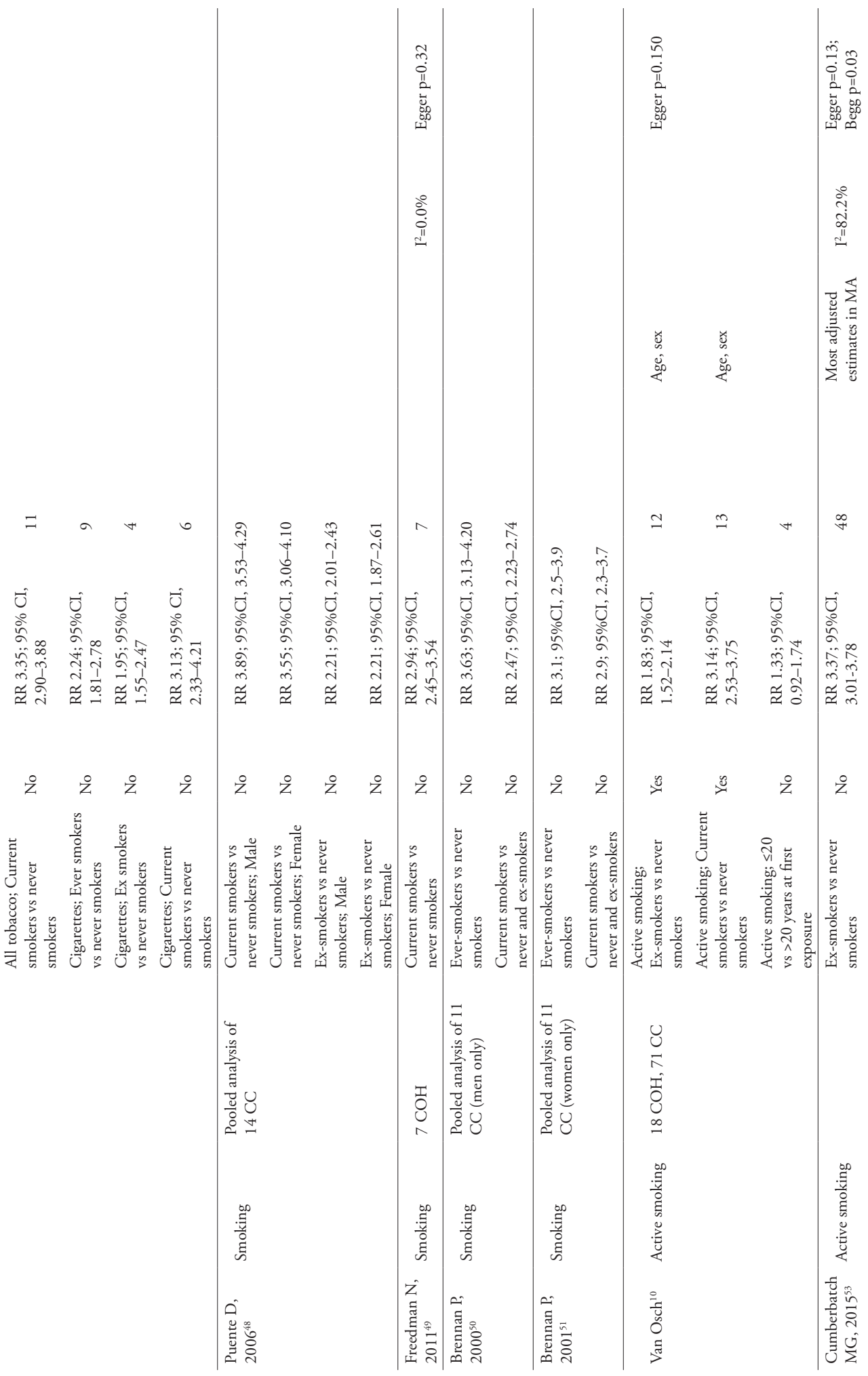


Chapter $\mathbf{5}$ | Modifiable risk factors for the prevention of bladder cancer

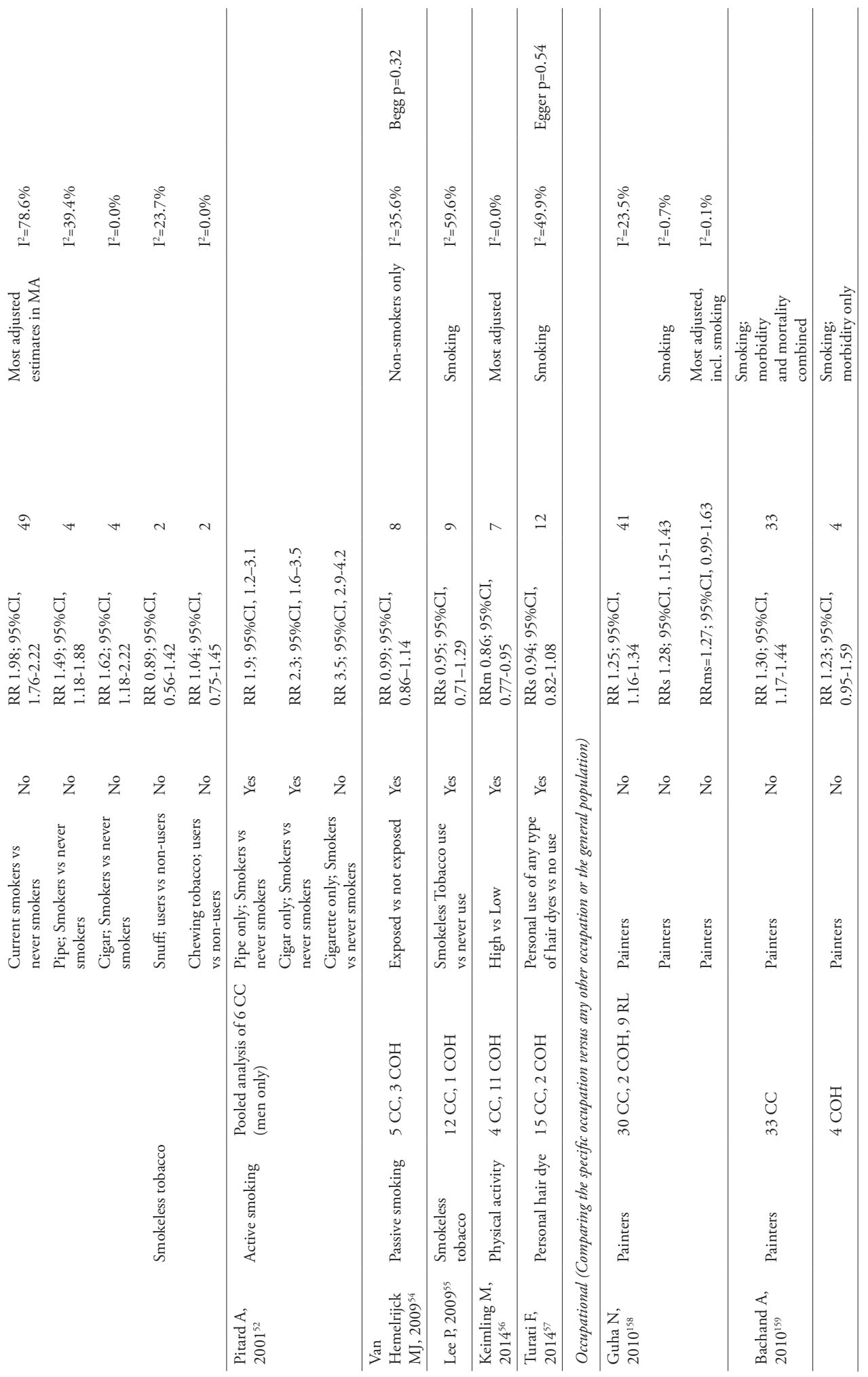


Chapter 5 I Modifiable risk factors for the prevention of bladder cancer

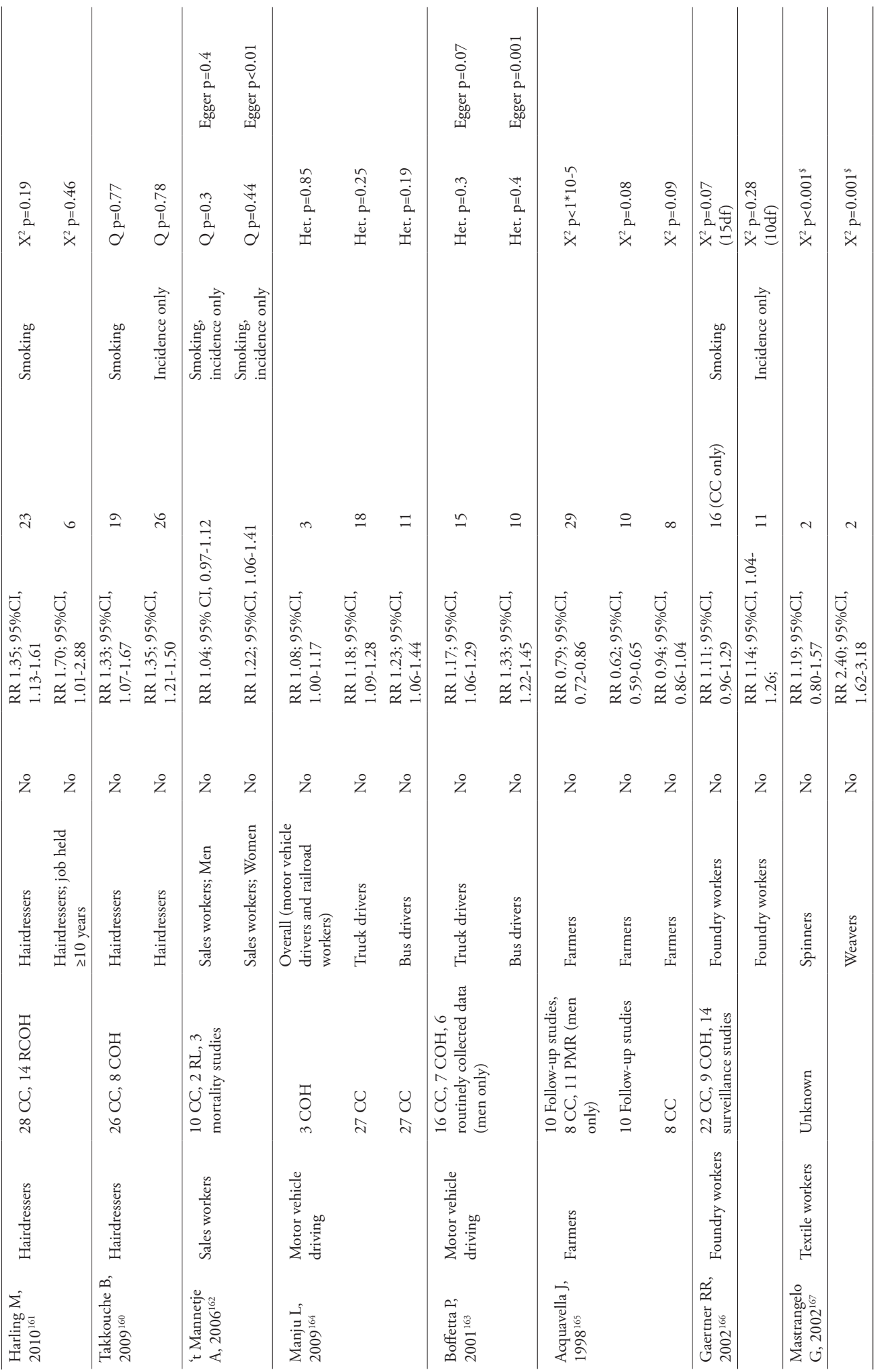


Chapter $\mathbf{5}$ I Modifiable risk factors for the prevention of bladder cancer

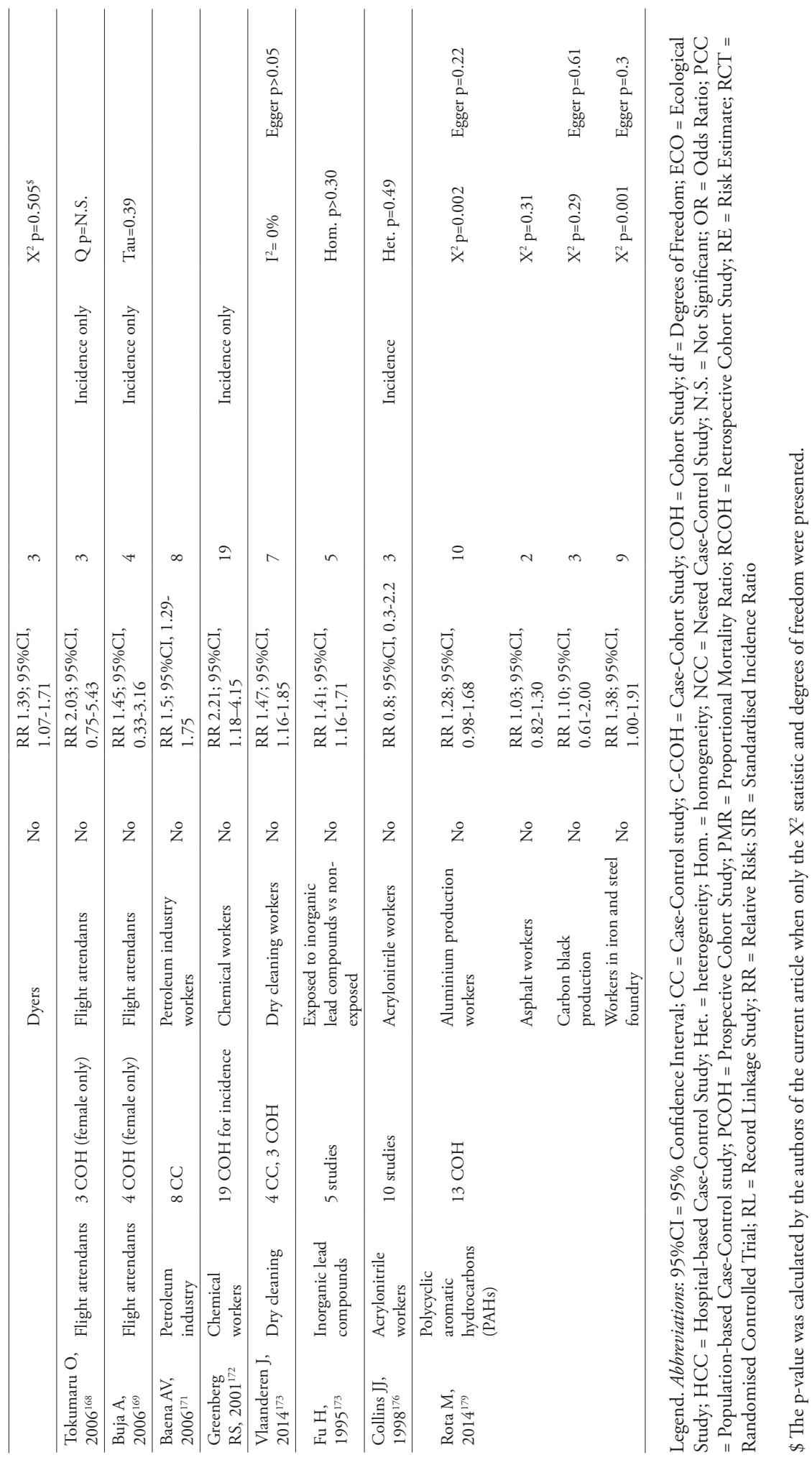


Chapter 5 I Modifiable risk factors for the prevention of bladder cancer

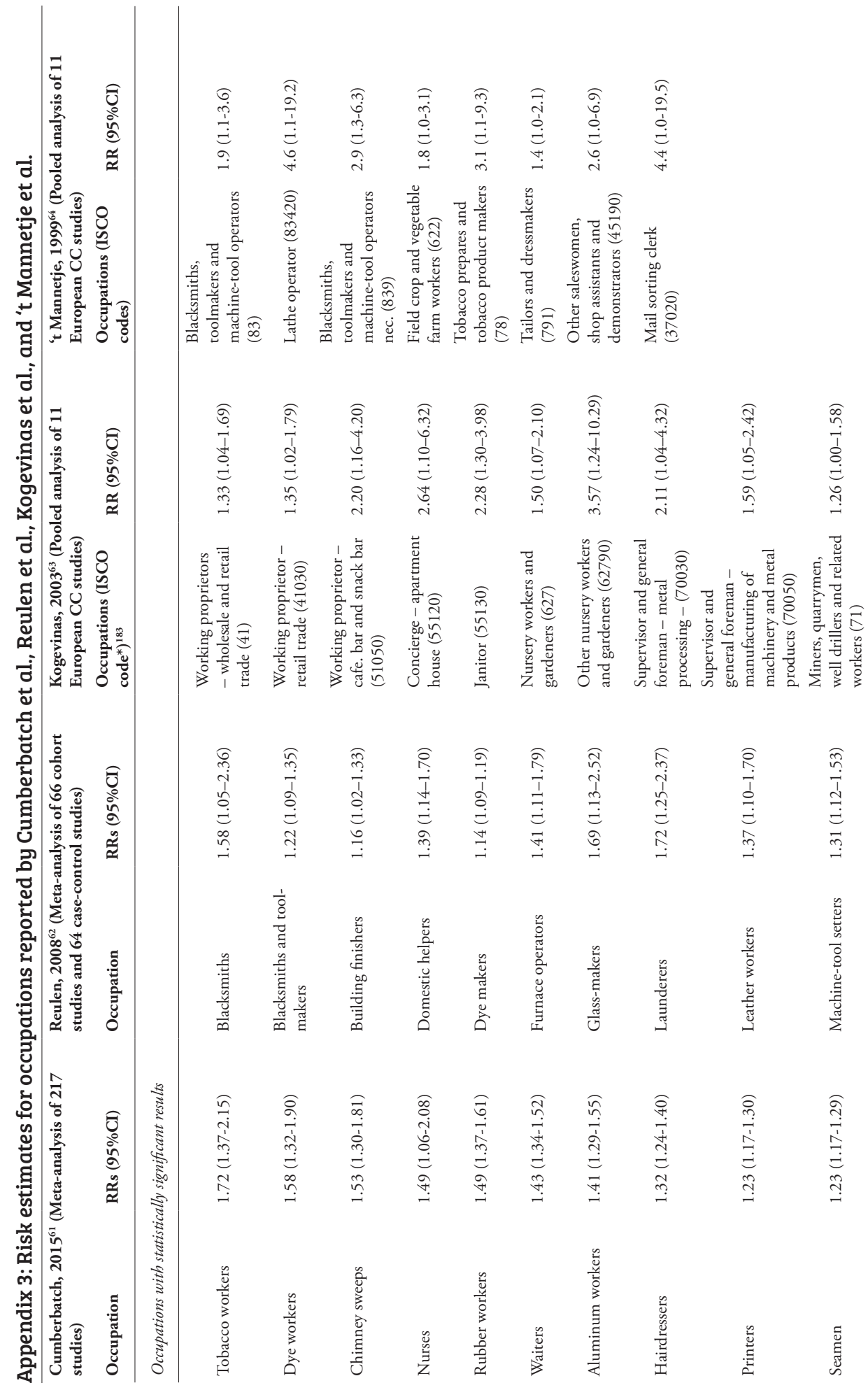




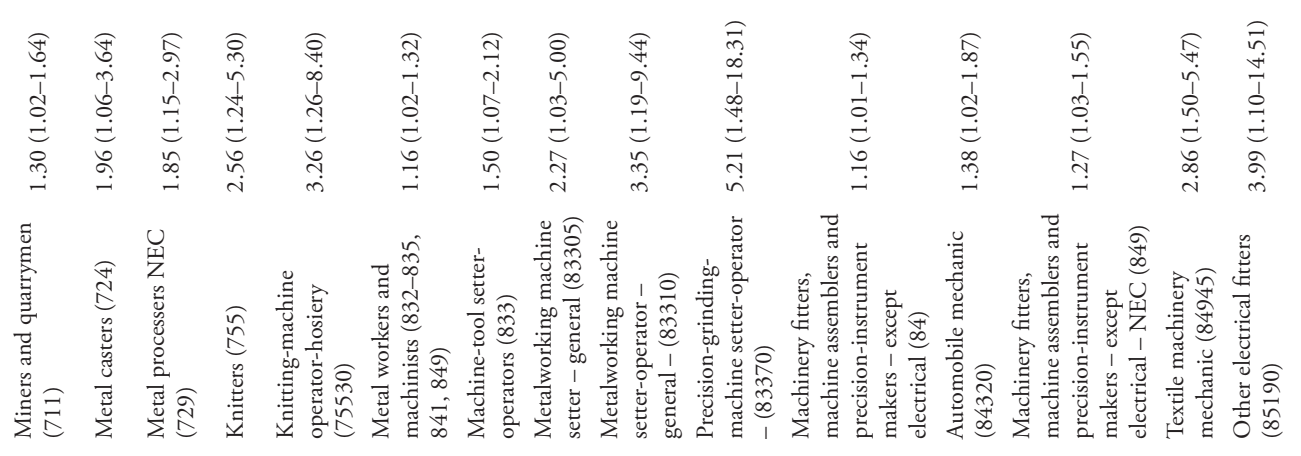

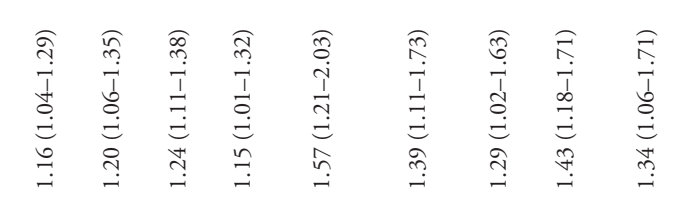

IIIt

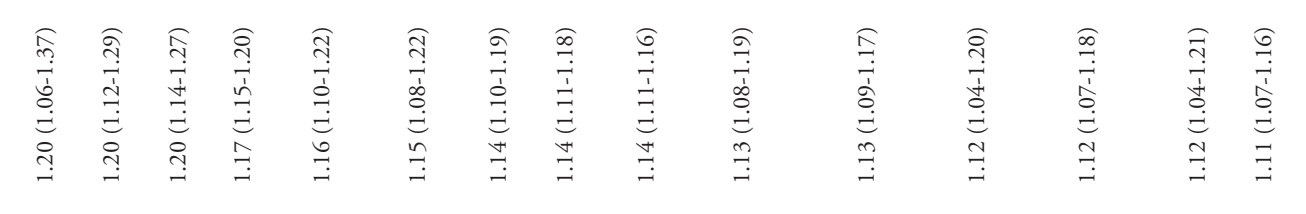
IIII

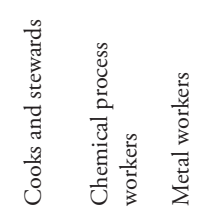
I

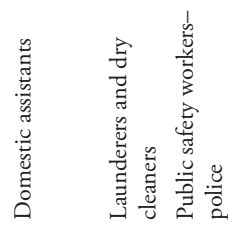




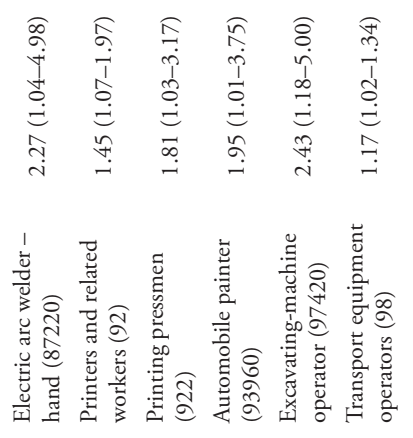

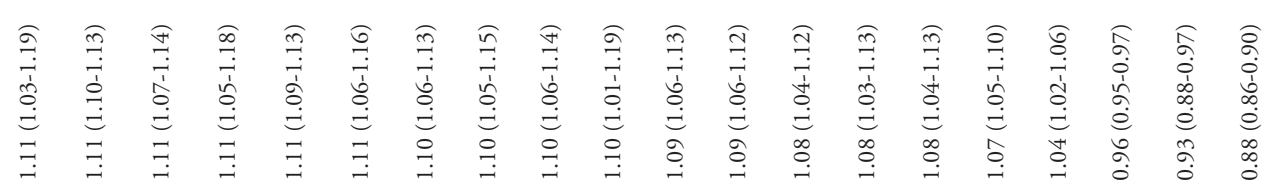

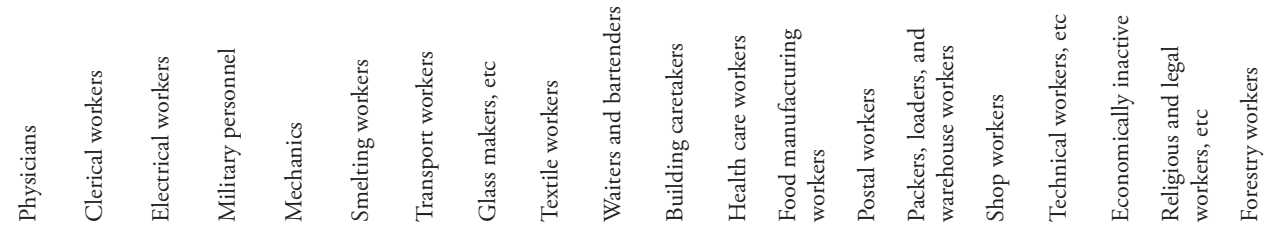


Chapter $\mathbf{5}$ | Modifiable risk factors for the prevention of bladder cancer

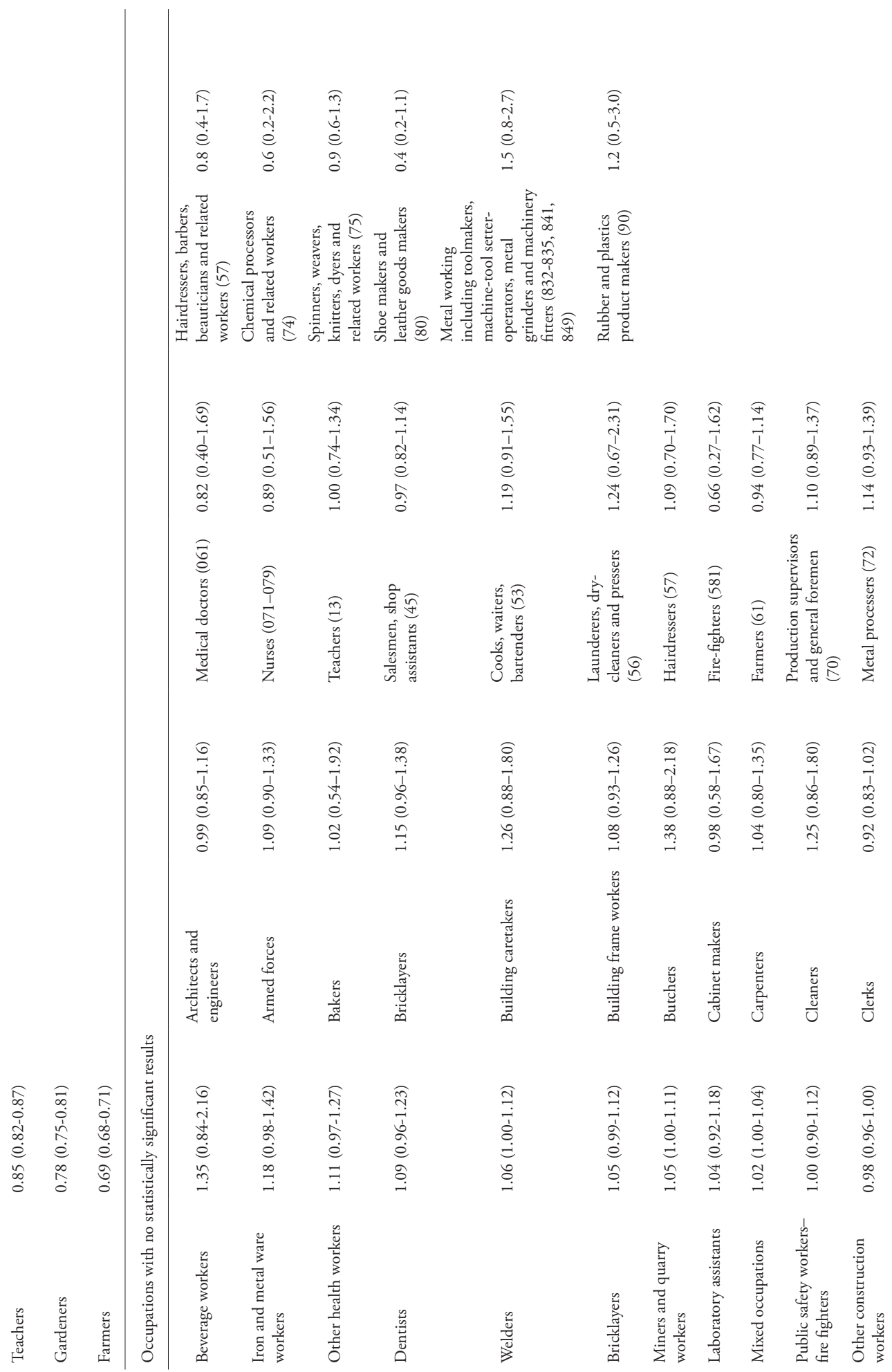




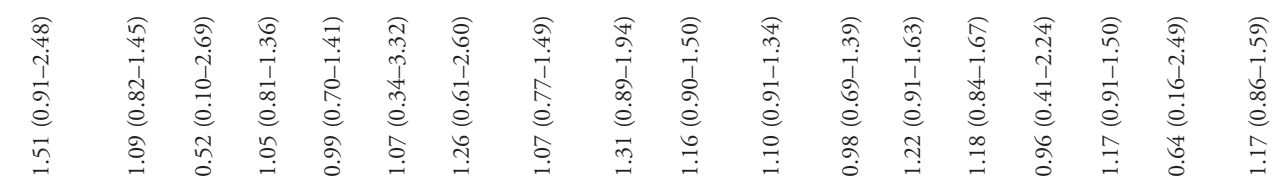

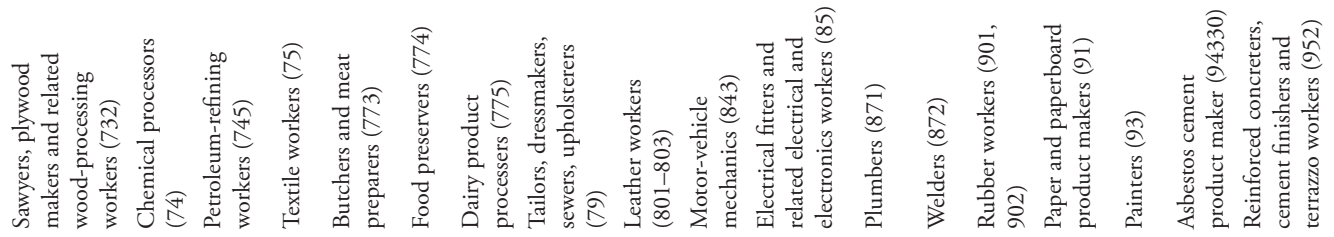

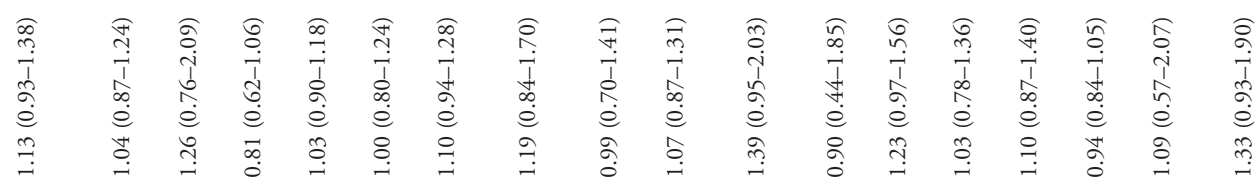

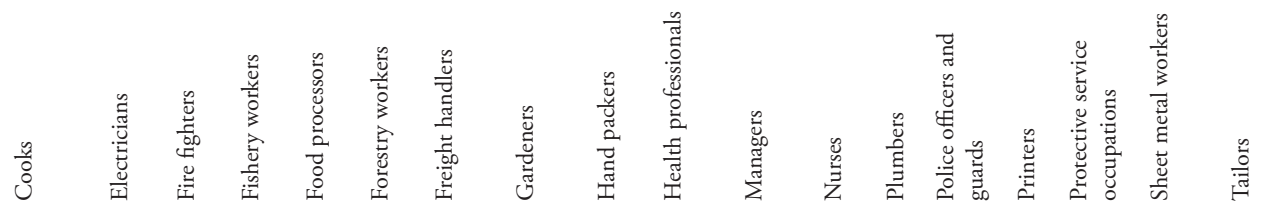

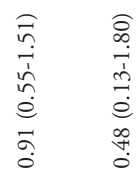

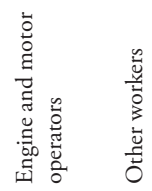



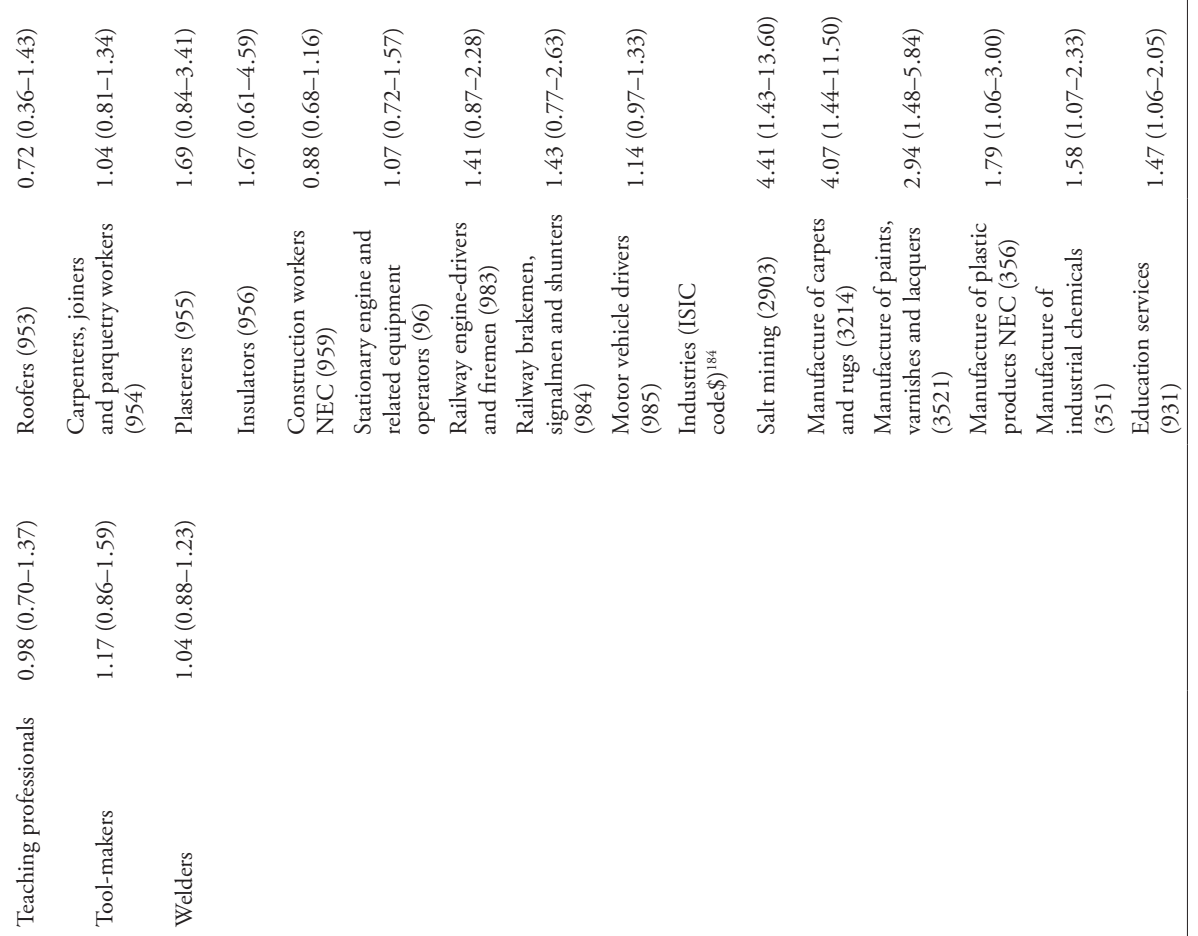


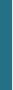




\section{Abstract}

Background: The incidence and prevalence of chronic diseases have reached epidemic proportions during the last decades and is not expected to diminish. Chronic diseases increasingly affect younger individuals too, with over $40 \%$ of all deaths due to non-communicable diseases occurring before the age of 70 . This has led to the development of information services aimed at preventive health care, such as Health Potential. ${ }^{\circ}$ This counselling service estimates a personal disease risk of a carefully selected list of preventable common chronic diseases that have both a genetic and a lifestyle component of development. The results are delivered faceto-face by a lifestyle counsellor, simultaneously stimulating initial steps towards behaviour change. This information can assist in lifestyle decision-making.

Methods: The primary aim is to study the effect of the Health Potential ${ }^{\oplus}$ service on change in lifestyle behaviour in distinguishable customer populations. The secondary aims are (1) to study the effect of the Health Potential ${ }^{\circ}$ service on determinants of behaviour change, (2) to study the effect of additional lifestyle counselling on behaviour change and determinants thereof, and (3) to describe characteristics of the Health Potential ${ }^{\bullet}$ customer. The study consists of two integrated designs: (A) a two-armed non-randomised controlled pre-test/post-test trial (1.5:1 ratio), followed by (B) a two-armed randomised controlled pre-test/ post-test trial (1:1 ratio), resulting in three study arms. Participants are clients of local prevention clinics, purchasing a personalised health check (PHC; intervention condition), consisting of Health Potential ${ }^{\circ}$ and a general health check, or the general health check alone (GHC; control condition) (Part A). PHC participants will be randomised to receive four additional lifestyle counselling sessions over a period of three months (Part B).

Discussion: This research can provide valuable insights in the effectiveness of and possible ways forward in the field of personalised prevention making use of lifestyle interventions enriched with modern genetic advancements.

Trial registration: www.trialregister.nl identifiers NTR6289 and NTR6288. Registered on 24 February 2017.

Key words: Personalised medicine, personalised prevention, trials, direct to consumer genetics, lifestyle epidemiology, genetic epidemiology, community genetics, health promotion. 


\section{Background}

The incidence and prevalence of chronic diseases have reached epidemic proportions during the last decades and is not expected to decrease or diminish. In 2012, 38 million people died as a result of a non-communicable disease, of which $82 \%$ were due to cardiovascular diseases, diabetes, cancer and chronic respiratory diseases. ${ }^{1}$ This number is projected to increase to 52 million deaths by $2030 .{ }^{2}$ Of particular concern is the fact that over $40 \%$ of all NCD-deaths occurred before the age of 70 in $2012 .{ }^{1}$ As a result of these developments, we must expect an increasing economic burden and reduction of quality of life in many societies, among both affected people and their social environment.

Most, if not all, common chronic diseases develop over a lifetime as a result of interplay between genetic predisposition and the accumulation of lifetime exposures to environmental and behavioural factors. According to the World Health Organisation (WHO), unhealthy diet, physical inactivity, tobacco use and harmful alcohol consumption are among the most important modifiable causes of chronic disease worldwide. ${ }^{3}$ For example, harmful alcohol use and tobacco use account for $12 \%$ of all deaths worldwide, and an additional $19 \%$ is accounted for by unhealthy diet and physical inactivity. ${ }^{3}$ As these are all modifiable, great potential exists for prevention or delay of chronic diseases, resulting in a renewed necessity for and interest in disease prediction and prevention. ${ }^{4}$

The fact that most chronic diseases seem to be preventable and that the neoliberal political climate in many countries have contributed to the rise of personal responsibility for health. ${ }^{5}$ Individuals are more and more expected to strive to obtain and maintain their best possible health. In order to do so, they need to be optimally informed and make their own risk-benefit analyses. This has led to an exponential growth in services aiming at assisting in informing and assisting individuals to make informed decisions. Another such approach is through genetic testing. ${ }^{6}$ Although one has no control over their genetic make-up, knowledge of genetic predispositions could provide the opportunity for personalised prevention strategies and may serve in health-related decision-making. ${ }^{7}$ 8

Personal genomic testing (PGT) uses genetic variations in individuals to estimate personal disease risks, and sometimes several other genetically determined phenotypes such as food intolerances or drug metabolism. ${ }^{9}$ PGT has become popular and accessible as a result of rapid advances in the field of DNA analysis techniques. This has made genome analysis possible at relatively low costs, with PGT companies like 23andMe dropping their price from $\$ 999$ in 2007 to $\$ 199$ in $2017 .{ }^{10,11}$ These services are 
mostly offered to the general population direct-to-consumer (DTC), meaning without interference of a physician. PGT may help individuals change their behaviour towards more health promoting lifestyle through identification of an increased disease risk. ${ }^{12}$ An important assumption of the success of this strategy is that the identification of increased disease risk could directly influence risk reduction behaviours. ${ }^{8,13,14}$ However, research has shown that this assumption mostly does not apply in all populations or that the effects are minor in general populations. ${ }^{14,15}$

Limitations of this idea that PGT may facilitate behaviour change are that, firstly, probability and genetic data are difficult to comprehend without counselling ${ }^{16,17}$ and understanding is strongly influenced by several factors such as the format of delivery ${ }^{18}$. Secondly, disease risks are not solely determined by either lifestyle or genetic factors, but both. ${ }^{19}$ Thirdly, disease risk information alone may not be motivating enough for individuals to change behaviour and therefore additional lifestyle intervention and motivational counselling may be necessary to help individuals change and maintain behaviour. $^{7}$

To address these shortcomings, a new disease risk information service has been developed: Health Potential ${ }^{\bullet}$. This service estimates a personal disease risk of a carefully selected list of preventable common chronic diseases that have both a genetic and a lifestyle component of development. The results are delivered face-to-face by a certified lifestyle counsellor, simultaneously stimulating initial steps towards behaviour change. Because previous research has shown that not all health care professionals feel confident in interpreting and communicating DTC-GT results to their patients or clients ${ }^{20,21}$, all Health Potential ${ }^{\circ}$ counsellors are trained to ensure confidence and skill.

The effect of this new personalised service will be researched in two integrated studies, one observational study and one intervention trial, comparing the service to a general health check only. The primary aim of the study is to study the effect of the Health Potential ${ }^{\odot}$ advice on change in lifestyle behaviour in distinguishable customer populations. The secondary aims are (1) to study the effect of the Health Potential ${ }^{\circ}$ service on determinants of behaviour change, (2) to study the effect of additional lifestyle counselling on behaviour change and determinants thereof, and (3) to study characteristics of the Health Potential ${ }^{\circ}$ customer. 


\section{Methods/design}

\section{Study design}

This study consists of two integrated trials. The first trial is a non-randomised twoarmed controlled pre-test/post-test superiority trial (Part A). Of one of those arms, participants can enter the second trial, which is a randomised controlled pre-test/posttest superiority trial (Part B), on the condition that they consent to participate. In order to obtain sufficient participants for part $B$, participants in part $A$ will be distributed in a 1.5:1 ratio. Participants will be randomised at a 1:1 ratio in part $B$. This results in three study arms (Figure 1). However, due to eligible participants of part A potentially not consenting to participation in part B, study arm 2 can again be considered as two study arms: participants in arm 2a have consented to participate in part B whereas participants in $2 \mathrm{~b}$ have not. (see Figure 1 for a visualisation)

\section{Rationale for the study design}

The study is designed in a way to reflect the 'real world' situation in which the participants are not randomised for part A and all participants pay a (reduced) fee for the services. The reason behind this approach is that we expect a certain level of 'pre-motivation' and possibly other different personal characteristics (e.g. personality, socio-economic status, income, level of education, gender, age) among clients of the Health Potential ${ }^{\circ}$ service, particularly when an additional 'barrier' exists of a fee that has to be paid for the service.

Due to the nature and cost of the products, and consistent with Roger's theory of early adopters $^{22}$, the study population is likely to consist of (apparently) healthy individuals aged 18 and over, expected to have higher social status and financial resources, higher education, and are relatively health conscious.

\section{Study setting}

Part A of the study takes place through online questionnaires, which participants can fill out at home. The additional lifestyle counselling will be

\section{Participants and recruitment}

Participants for part A of the study will be recruited from the regular customers at local prevention clinics who have purchased one of the following products:

- Product 1: Personalised Health Check (PHC). The PHC consists of the Health Potential" service (future health) and a general health check of the customer's current health.

- Product 2: A general health check of current health only (GHC). 
Chapter 6 | Stages of behavioural change after DTC-GT disease risk profiling: study protocol

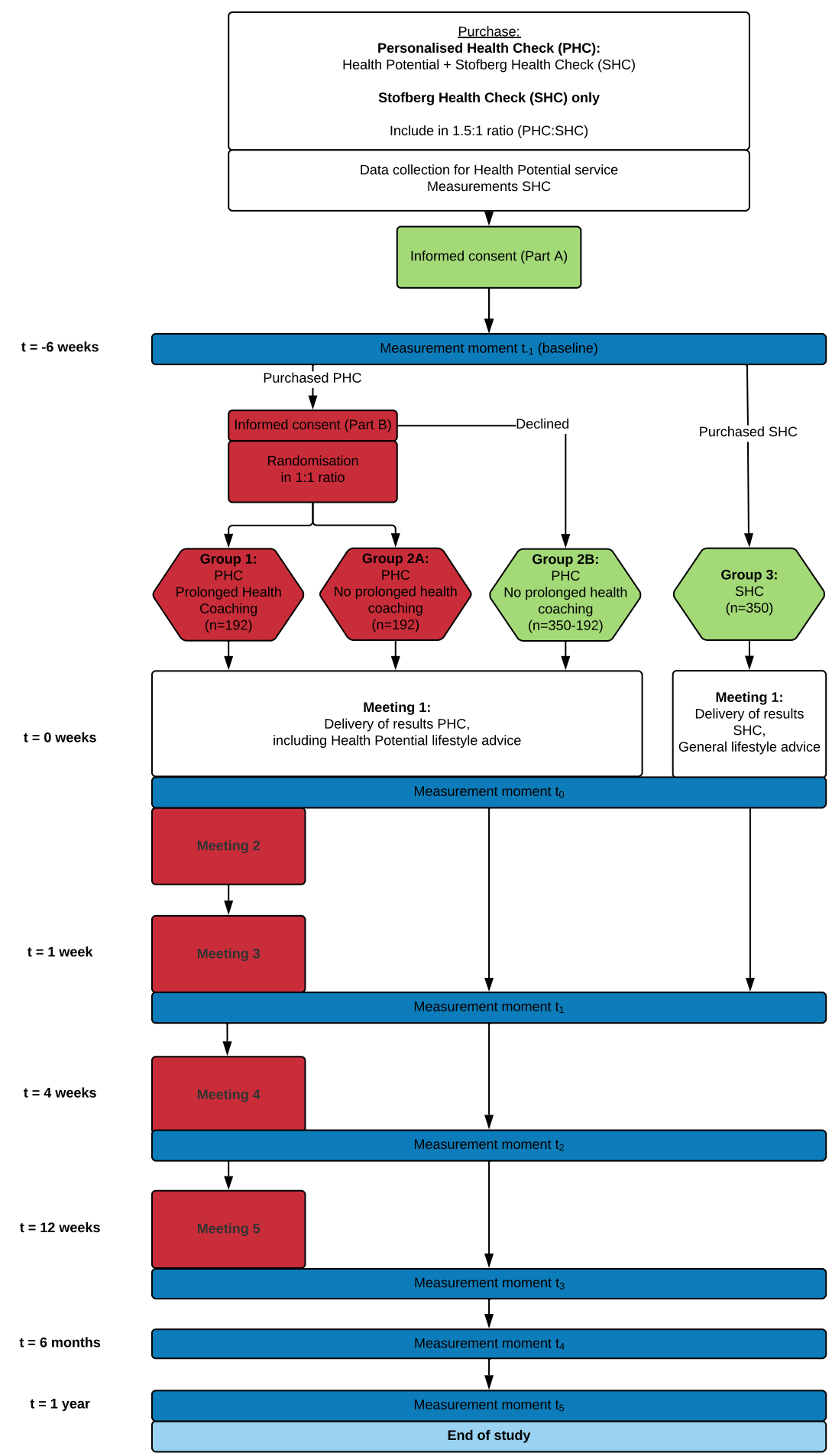

Figure 1 Flow chart of the study

offered through certified counsellors located in South-Limburg, The Netherlands. 
Potential participants will be informed of the study by the local responsible investigator of each clinic. Eligible participants for part A are apparently healthy individuals aged 18 and over, with a Dutch language level similar to language proficiency level B2, not currently following any prescribed dietary or other guidelines, not pregnant or trying to become pregnant, authorised to make autonomous decisions, able to independently visit the counselling clinic, and have internet access and an email address. Participants who purchased product 1 are eligible to take part in part B of the study. Enrolment will be open for one year.

\section{Sample size and power calculation}

The sample size calculation is based on the proportion of participants who have changed their behaviour (being in the action/maintenance stage of behaviour change, see 'study parameters' below) for at least 3 of the received advices at follow-up in the overall analysis. The type-I error rate was set at 0.05 and an additional $20 \%$ was added to compensate for loss-to-follow-up in part A.

Part A: The smallest difference is expected between arms 2 and 3. It is estimated that with $(291+20 \%=) 350$ participants in each arm, part A has a power of $80 \%$ to detect a proportion of 0.3 in arm 2 versus 0.2 in arm 3 .

Part B: Study arm 1 will be compared to arm 2a. It is then estimated that with $(160+20 \%=) 192$ participants in each arm, part B has a power of $80 \%$ to detect a proportion of 0.45 in arm 1 versus a proportion of 0.3 in arm 2a. As in part A 548 participants will be included in the PHC condition, this will gives a margin of (548$384=) 164$ for participants not consenting to participate in part $\mathrm{B}$. Together this results in a total number of participants of $(192+350+350=) 892$.

\section{Blinding, randomisation and treatment allocation}

Participants who purchased the PHC and who consented to participate in part B will be electronically randomised to receive the additional lifestyle counselling. Randomisation will be stratified by local clinic and block randomisation will be used with block sizes of 4 within each strata. The health coach will subsequently implement the appropriate intervention.

Although, blinding is not possible for the participants and the health counsellors delivering the intervention, the data analyses will be performed in a blinded format through preparation of the dataset by an independent researcher. 


\section{Investigational behavioural intervention}

Part A does not contain an intervention. In part B the participant receives additional lifestyle counselling. The lifestyle counselling consists of four additional personal faceto-face sessions with a purpose-trained lifestyle counsellor. The counselling follows four phases matching four core question ${ }^{23}$ and is delivered using principles of motivational interviewing 24,25 :

- Counselling phase 1: "Am I at an increased risk because of my lifestyle?"

- Counselling phase 2: "Which choices are suitable for me?"

- Counselling phase 3: "How do I translate my choices to behaviour?"

- Counselling phase 4: "How can I maintain my change in behaviour?"

All lifestyle counselling sessions are done by the same certified lifestyle counsellors for one participant. The sessions take place at $\mathrm{t}=0, \mathrm{t}=1 \mathrm{w}, \mathrm{t}=4 \mathrm{w}$ and $\mathrm{t}=12 \mathrm{w}$ (see Figure 1; sessions 2-5) and will last approximately 45-60 minutes per session.

\section{Study parameters}

To study the effect of Health Potential on behaviour change, a conceptual framework has been developed (Figure 2), based on a previously proposed framework for PGT by Bloss et al. ${ }^{8}$ and the I-change model ${ }^{26}$. The framework consists of two steps that are relevant for eventual behaviour change: (1) purchasing Health Potential and (2) behaviour change as a result of Health 
Chapter 6 | Stages of behavioural change after DTC-GT disease risk profiling: study protocol

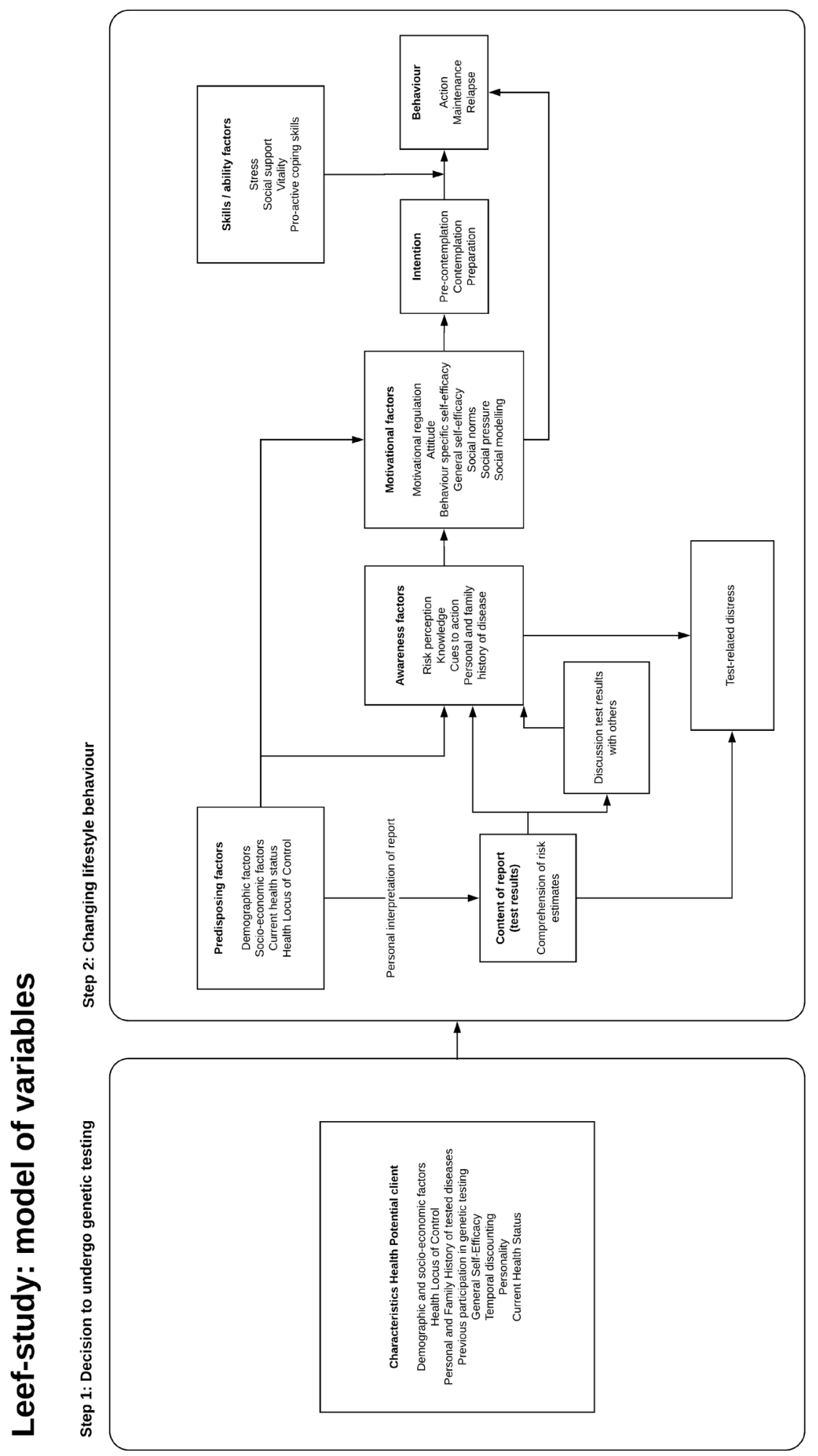

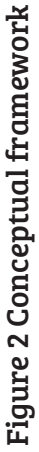




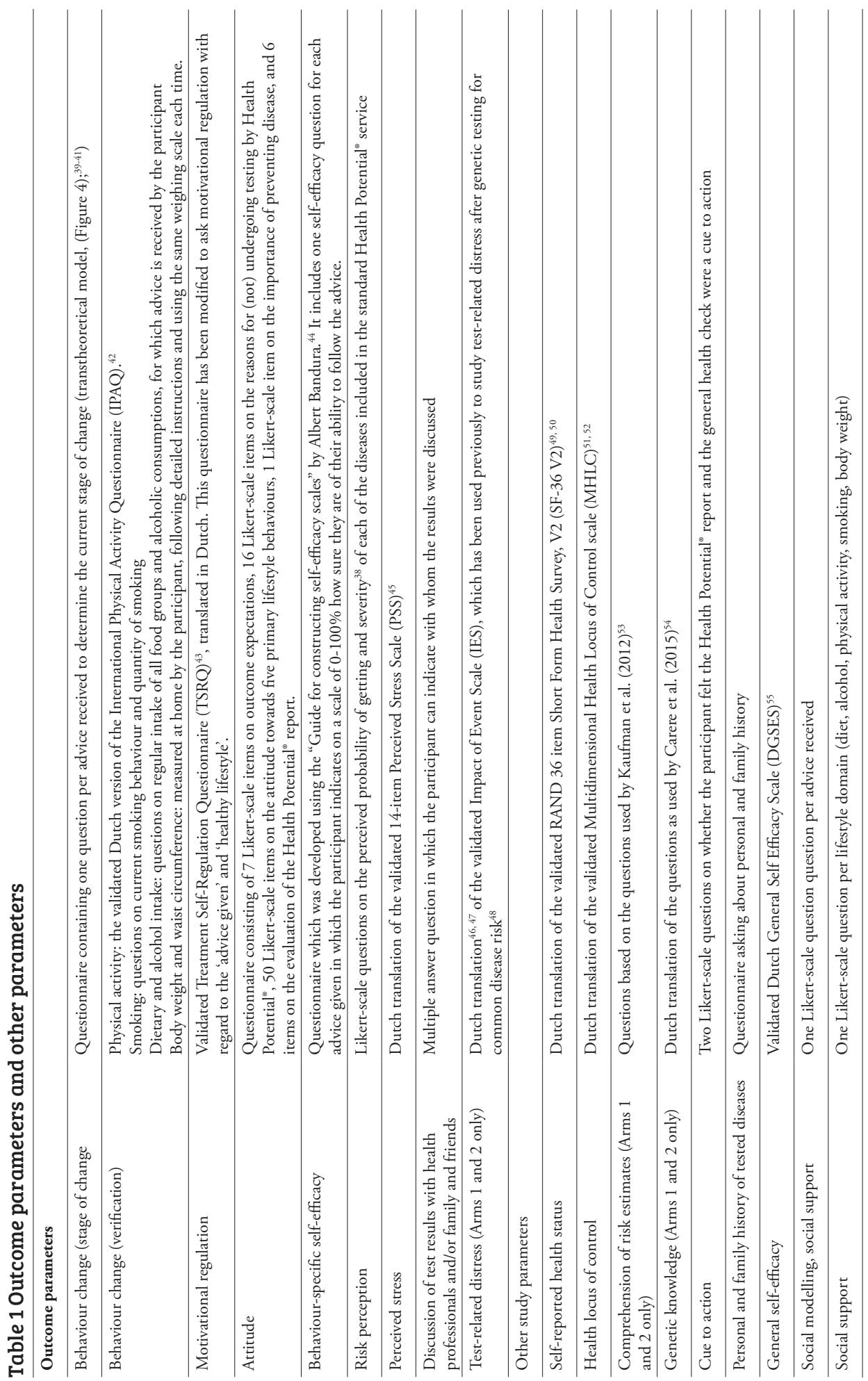


Chapter 6 | Stages of behavioural change after DTC-GT disease risk profiling: study protocol

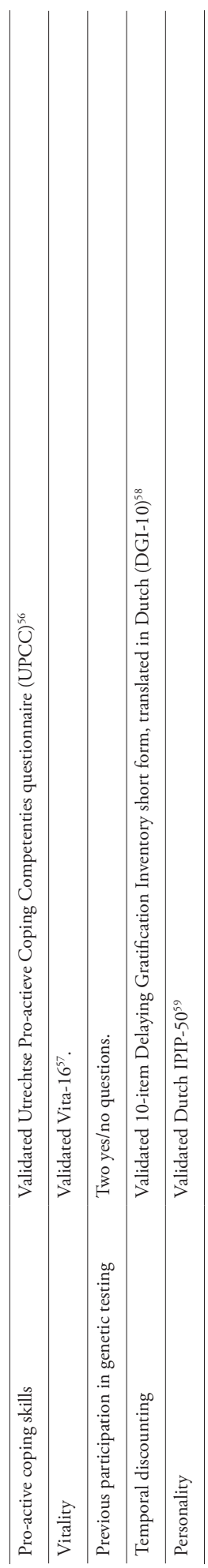


Chapter 6 | Stages of behavioural change after DTC-GT disease risk profiling: study protocol

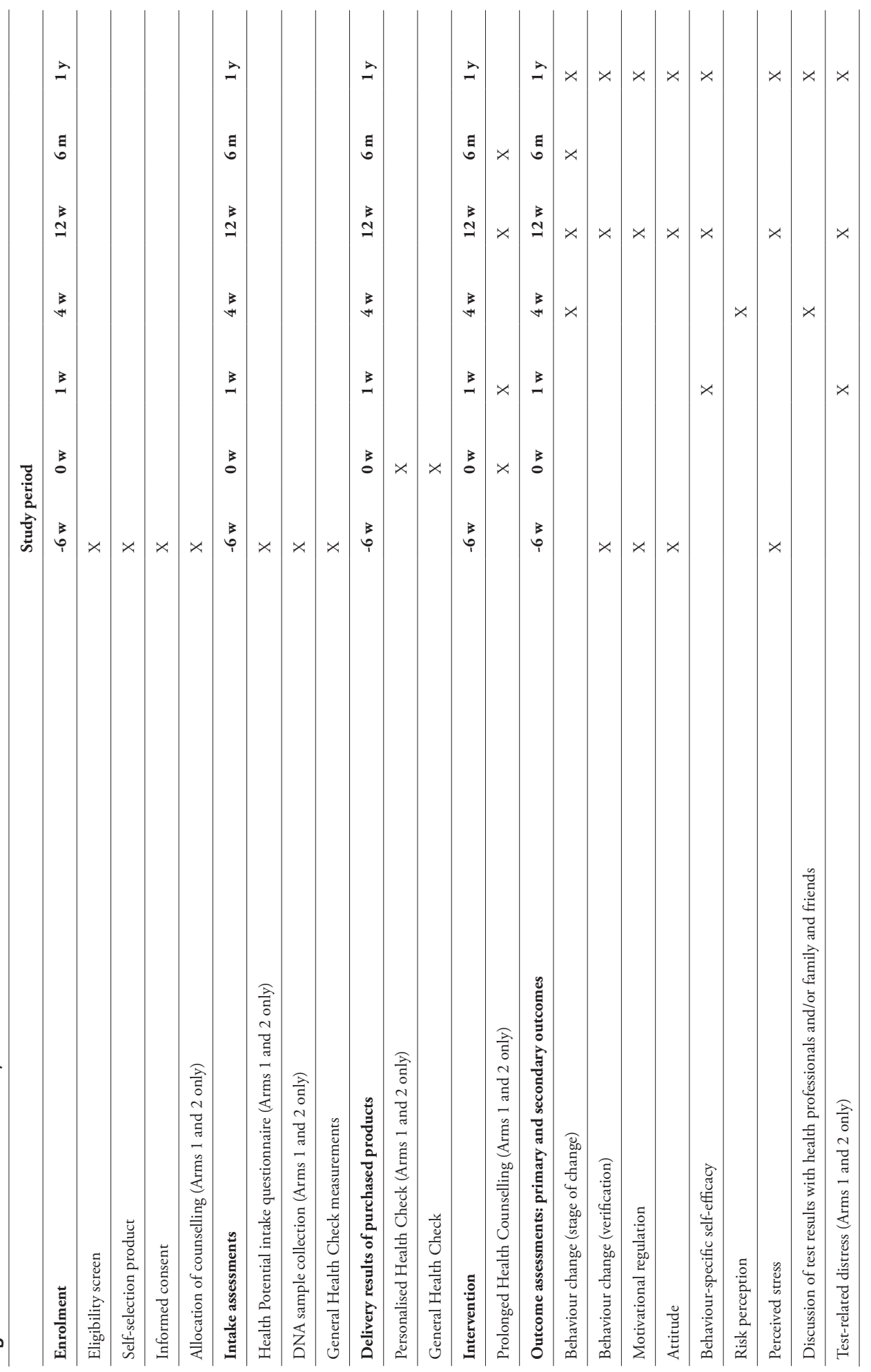


Chapter 6 | Stages of behavioural change after DTC-GT disease risk profiling: study protocol

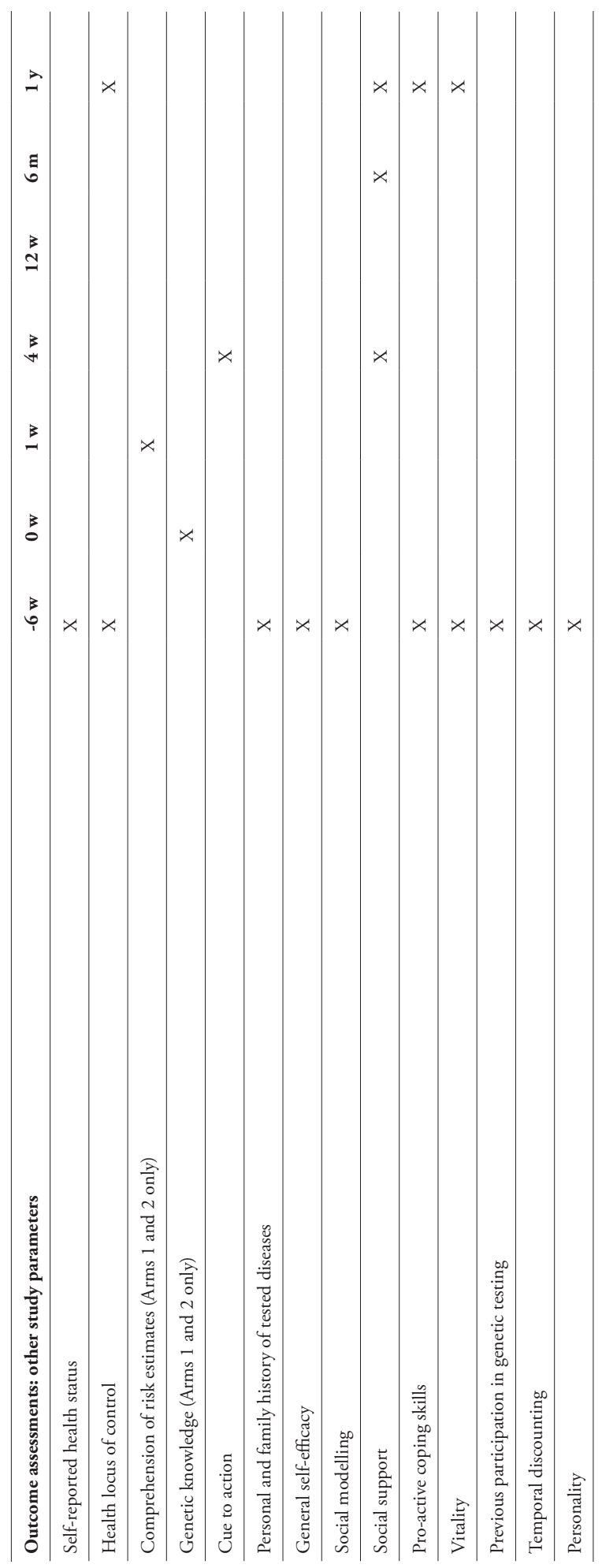




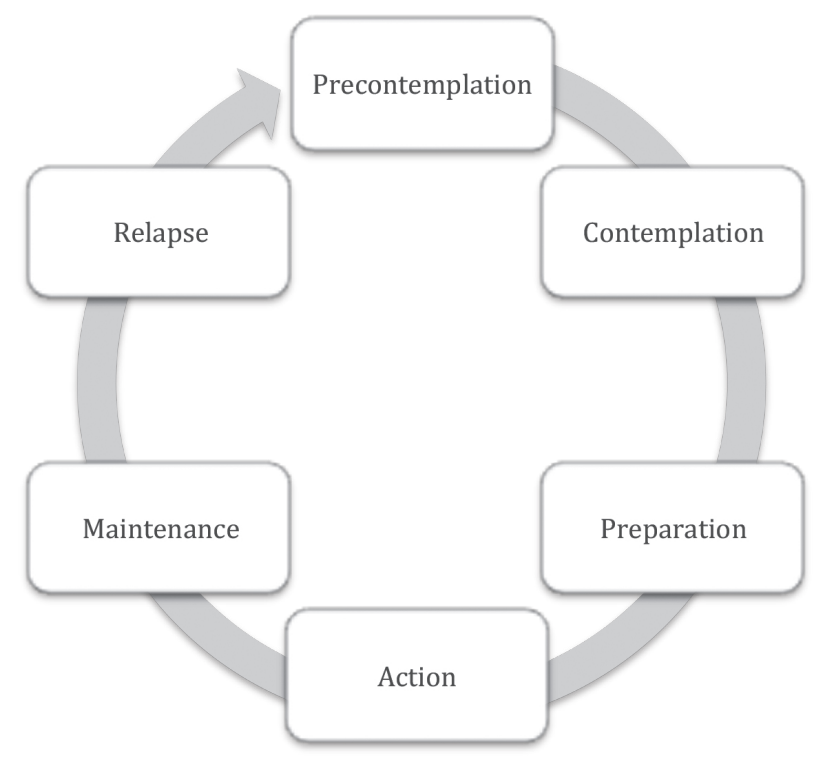

Figure 4 Transtheoretical model $\mathrm{l}^{39-41}$

Potential. Determinants included in both steps are based on the literature on PGT $21,27-37$ and the I-change model $^{38}$.

All parameters (Table 1) are measured using online questionnaires, as part of part A of the study, and are saved in a coded format. Measurements take place at $\mathrm{t}=-6 \mathrm{w}$ (baseline), $\mathrm{t}=0, \mathrm{t}=1 \mathrm{w}, \mathrm{t}=4 \mathrm{w}, \mathrm{t}=12 \mathrm{w}, \mathrm{t}=6 \mathrm{~m}$, and $\mathrm{t}=1 \mathrm{y}$ (Figure 3 ).

\section{Statistical analysis}

Where appropriate, the analyses will be performed according to an intention-to-treat (ITT) approach (part B only) and per-protocol approach. Data will be analysed using STATA (Stata Corp. Stata Statistical Software. College Station, TX: StataCorp LP). Statistical significance is assumed at a p-value of 0.05 and no correction for multiple testing will be applied.

\section{Ethical considerations}

Due to the nature of the researched population and the very limited risks associated with the study, no Data Safety Monitoring Board or Safety Committee is deemed necessary. No interim analyses will be performed.

The full protocols, for both part A and B, can be found on http://dataverse.nl/ under identifier 10411/XKOM8H 
Chapter 6 I Stages of behavioural change after DTC-GT disease risk profiling: study protocol

\section{Discussion}

To our best knowledge, this is the first trial investigating such a wide range of determinants with regard to the effect of a disease prevention service based on both genetic and lifestyle components of disease risk. The study will contribute to the debate on whether genetic prevention services can be effective in improving lifestyle behaviour in certain customer groups and will help identify actionable determinants that contribute to an increased effect. Health Potential ${ }^{\oplus}$ will support the individual in making informed decisions about their health and lifestyle, which will become increasingly important in the current paradigm of personal responsibility for one's health.

The study has several strengths and risks. Firstly, the pragmatic approach of the study will give a good indication of the effect in a real-life situation after implementation, resulting in small theory-practice gap. However, it may also result in greater heterogeneity and thereby a dilution of the effect. On the other hand, the expected population of mostly early adopters might again reduce heterogeneity. Secondly, due to this pragmatic approach and the relatively high cost of genetic testing, the study population can be expected to be a selective group of higher educated individuals with a relatively high income. As health and lifestyle are associated with socio-economic position, services like Health Potential ${ }^{\circ}$ may contribute to increasing health inequalities when the test is purchased personally. In the future, employers or municipalities may subsidise such tests for their employees or certain groups of inhabitants, which could diminish this effect. Finally, the outcomes include a wide range of actionable determinants of behaviour change, which will allow us to investigate through which determinants the effect on behaviour change is achieved. Knowing these explanatory variables may then inform us about the determinants that need to be addressed further in order to achieve a greater effect on behaviour change, direct recruitment strategies, and guide further development of genetic testing services. In addition, identification of specific groups of customers in which an effect is or is not seen, will further help to tailor strategies.

PGT services, such as Health Potential ${ }^{\circ}$, also carry some risks and benefits for the consumer. Firstly, because of the high psychological impact of genetic testing for diseases with a strong genotype-phenotype interaction, it is under debate whether genetic testing for common disease risk also leads to too high levels of distress among participants. However, recent studies have suggested that the level of distress is minimal. $7,15,48,60$ The close contact between client and coach is hypothesised further reduce any worry or distress and in case any serious distress arises, this can be recognised at an early stage and appropriate action can be taken. Whether this holds true will also be examined in this study. Secondly, a serious risk of PGT services is misinterpretation of test results 
Chapter 6 | Stages of behavioural change after DTC-GT disease risk profiling: study protocol

by consumers. ${ }^{17}$ Consequences of misinterpretation may be undermined motivation (e.g. interpreting a reduced risk as definitely not getting the disease), unnecessary worry and distress, and a burden on the health care system through requesting unnecessary medical tests and asking for help from health care professionals to interpret the results. ${ }^{7}$, ${ }^{61}$ To address the issue, the Health Potential ${ }^{\bullet}$ report has been based on literature on communication of disease risks ${ }^{62}$ and the results are explained to the client face-to-face by a certified Health Potential ${ }^{\circ}$ coach. Finally, Health Potential ${ }^{\circ}$ has a strong connection with her end users, including both coaches and clients. In the development of the supporting software and the communication of results, end users have been involved. This involvement is crucial for acceptance and therefore viability of a service. ${ }^{63}$

This research can provide valuable insight in the effectiveness of PGT services. These findings can be used in the development and improvement of lifestyle interventions enriched with modern genetic advancements. 


\section{Trial status}

The trial is currently in preparation.

List of abbreviations and relevant definitions

\begin{tabular}{ll}
\hline BMI & Body Mass Index \\
\hline DTC & Direct-to-Consumer \\
\hline PGT & Personal Genomic Testing \\
\hline METC & $\begin{array}{l}\text { Medical research ethics committee (MREC); in Dutch: medisch ethische } \\
\text { toetsing commissie (METC) }\end{array}$ \\
\hline PHC & Personal Health Check \\
\hline GHC & General Health Check \\
\hline WHO & World Health Organisation \\
\hline
\end{tabular}

\section{Declarations}

\section{Ethics approval and consent to participate}

The protocol has been approved by the METC AzM/UM (numbers 16-4-077 and 163014) and is registered in www.trialregister.nl on 24 February 2017 with registration numbers NTR6289 and NTR6288.

Informed consent will be obtained from each participant for both part A and part B separately.

\section{Consent for publication}

Not applicable

\section{Availability of data and material}

Data sharing is not applicable to this article as no datasets were generated or analysed during the current study.

\section{Competing interests}

Health Potential may become a spin off service from Maastricht University led by Prof. Maurice Zeegers.

\section{Funding}

This project was funded by the Maastricht University Interfaculty Programme 'Eatwell'. 
Chapter 6 | Stages of behavioural change after DTC-GT disease risk profiling: study protocol

The funder had no influence in development of the protocol.

\section{Author's contributions}

All authors (KS, AW, AS, and MZ) contributed substantially to the conception and design of the study and ethical submissions. KS drafted the manuscript, which was critically revised by AW, MZ and AS. AW, AS and MZ obtained funding. All authors read and approved the final manuscript, and have given approval for publication of this paper.

\section{Acknowledgements}

The authors wish to thank Stofberg Group for their assistance in planning the logistical aspects of the study and Operis for their support in the certification course of the Health Potential ${ }^{\circ}$ counsellors. 
Chapter 6 | Stages of behavioural change after DTC-GT disease risk profiling: study protocol

\section{References}

1. Alwan A. Global status report on noncommunicable diseases 2010. World Health Organization, 2011.

2. World Health Organization. Projections of mortality and causes of death, 2015 and 2030.

3. World Health Organization. Global health risks: mortality and burden of disease attributable to selected major risks. World Health Organization, 2009.

4. Singh AR. Modern Medicine: Towards Prevention, Cure, Well-being and Longevity. Mens Sana Monographs. 2009; 8: 11.

5. Resnik DB. Responsibility for health: personal, social, and environmental. Journal of medical ethics. 2007; 33: 444-5.

6. Resnik DB. Genetics and Personal Responsibility for Health. New genetics and society. 2014; 33: $113-25$.

7. Stewart K, Schreurs M, Wesselius A, Schols A and Zeegers M. Behavioural changes and psychological responses after receiving direct-to-consumer genetic test results: a systematic review and meta-analysis (in press). Journal of community genetics. 2017.

8. Bloss CS, Madlensky L, Schork NJ and Topol EJ. Genomic information as a behavioral health intervention: can it work? Personalized medicine. 2011; 8: 659-67.

9. Gollust SE, Wilfond BS and Hull SC. Direct-to-consumer sales of genetic services on the Internet. Genetics in medicine : official journal of the American College of Medical Genetics. 2003; 5: 3327.

10. Tsotsis A. Another $\$ 50 M$ Richer, 23andMe Drops Its Price To $\$ 99$ Permanently. But Will The Average Dude Buy In? : TechCrunch, December 11, 2012.

11. 23andMe.

12. Offit K. Personalized medicine: new genomics, old lessons. Human genetics. 2011; 130: 3-14.

13. Henrikson NB, Bowen D and Burke W. Does genomic risk information motivate people to change their behavior. Genome Med. 2009; 1: 37.

14. Leventhal H, Benyamini $\mathrm{Y}$, Brownlee $S$, et al. Illness representations: theoretical foundations. Perceptions of health and illness. 1997; 2: 19-46.

15. Roberts JS and Ostergren J. Direct-to-consumer genetic testing and personal genomics services: a review of recent empirical studies. Current genetic medicine reports. 2013; 1: 182-200.

16. Lanie AD, Jayaratne TE, Sheldon JP, et al. Exploring the public understanding of basic genetic concepts. Journal of genetic counseling. 2004; 13: 305-20.

17. McGrath SP, Coleman J, Najjar L, Fruhling A and Bastola DR. Comprehension and Data-Sharing Behavior of Direct-To-Consumer Genetic Test Customers. Public health genomics. 2016; 19: 11624.

18. Fausset CB. Comprehension of health risk probabilities: the roles of age, numeracy, format, and mental representation. 2012.

19. Al-Zalabani AH, Stewart KFJ, Wesselius A, Schols MWJ and Zeegers MP. Modifiable risk factors for the prevention of bladder cancer: a systematic review of meta-analyses European Journal of Epidemiology. 2016; 31: 811-51.

20. Christensen KD, Vassy JL, Jamal L, et al. Are physicians prepared for whole genome sequencing? A qualitative analysis. Clinical Genetics. 2016; 89: 228-34.

21. Goldsmith L, Jackson L, O'Connor A and Skirton H. Direct-to-consumer genomic testing: systematic review of the literature on user perspectives. European Journal of Human Genetics. 2012; 20: 811-6.

22. Rogers EM. Diffusion of Innovations. 5th Edition ed.: Simon and Schuster, 2003.

23. Lak M and Bijma M. Leefstijlcoaching: kernvragen bij gedragsverandering. Bohn Stafleu van Loghum, 2012.

24. Miller WR. Motivational interviewing with problem drinkers. Behavioural psychotherapy. 1983; 11: $147-72$. 
Chapter 6 | Stages of behavioural change after DTC-GT disease risk profiling: study protocol

25. Rubak S, Sandbæk A, Lauritzen T and Christensen B. Motivational interviewing: a systematic review and meta-analysis. British Journal of General Practice. 2005; 55: 305-12.

26. De Vries H, Mesters I, van't Riet J, Willems K and Reubsaet A. Motives of Belgian adolescents for using sunscreen: the role of action plans. Cancer Epidemiology Biomarkers \& Prevention. 2006; 15: 1360-6.

27. Vayena E, Ineichen C, Stoupka E and Hafen E. Playing a Part in Research University Students' Attitudes to Direct-To-Consumer Genomics. Public health genomics. 2014; 17: 158-68.

28. Sweeny K and Legg AM. Predictors of interest in direct-to-consumer genetic testing. Psychology \& health. 2011; 26: 1259-72.

29. Cherkas LF, Harris JM, Levinson E, Spector TD and Prainsack B. A survey of UK public interest in internet-based personal genome testing. 2010.

30. Su Y, Howard HC and Borry P. Users' motivations to purchase direct-to-consumer genome-wide testing: an exploratory study of personal stories. Journal of community genetics. 2011;2: 135-46.

31. Vayena E, Gourna E, Streuli J, Hafen E and Prainsack B. Experiences of early users of direct-toconsumer genomics in Switzerland: an exploratory study. Public health genomics. 2012; 15: 35262.

32. McGowan ML, Fishman JR and Lambrix MA. Personal genomics and individual identities: motivations and moral imperatives of early users. New Genetics and Society. 2010; 29: 261-90.

33. McBride CM, Alford SH, Reid RJ, Larson EB, Baxevanis AD and Brody LC. Characteristics of users of online personalized genomic risk assessments: implications for physician-patient interactions. Genetics in Medicine. 2009; 11: 582-7.

34. Gollust SE, Gordon ES, Zayac C, et al. Motivations and perceptions of early adopters of personalized genomics: perspectives from research participants. Public health genomics. 2012; 15: 22-30.

35. Mavroidopoulou V, Xera E and Mollaki V. Awareness, attitudes and perspectives of direct-toconsumer genetic testing in Greece: a survey of potential consumers. Journal of human genetics. 2015; 60: 515-23.

36. McGuire AL, Diaz CM, Wang T and Hilsenbeck SG. Social networkers' attitudes toward direct-toconsumer personal genome testing. The American journal of bioethics. 2009; 9: 3-10.

37. Bloss CS, Ornowski L, Silver E, et al. Consumer perceptions of direct-to-consumer personalized genomic risk assessments. Genetics in Medicine. 2010; 12: 556-66.

38. de Vries H, Mesters I, Van de Steeg H and Honing C. The general public's information needs and perceptions regarding hereditary cancer: an application of the Integrated Change Model. Patient education and counseling. 2005; 56: 154-65.

39. Prochaska JO, DiClemente CC, Velicer WF, Ginpil S and Norcross JC. Predicting change in smoking status for self-changers. Addictive behaviors. 1985; 10: 395-406.

40. Prochaska JO and Goldstein MG. Process of smoking cessation. Implications for clinicians. Clinics in chest medicine. 1991; 12: 727-35.

41. Prochaska JO and Velicer WF. The transtheoretical model of health behavior change. American journal of health promotion. 1997; 12: 38-48.

42. Craig CL, Marshall AL, Sjostrom M, et al. International physical activity questionnaire: 12-country reliability and validity. Medicine and science in sports and exercise. 2003; 35: 1381-95.

43. Ryan RM and Connell JP. Perceived locus of causality and internalization: examining reasons for acting in two domains. Journal of personality and social psychology. 1989; 57: 749.

44. Bandura A. Guide for constructing self-efficacy scales. Self-efficacy beliefs of adolescents. 2006; 5.

45. Cohen S, Kamarck T and Mermelstein R. A global measure of perceived stress. Journal of health and social behavior. 1983: 385-96.

46. Brom D and Kleber RJ. De Schok Verwerkings Lijst [The Dutch version of the Impact of Event Scale]. Nederlands Tijdschrift voor de Psychologie. 1985; 40: 164-8.

47. van der Ploeg E, Mooren T, Kleber RJ, van der Velden PG and Brom D. Construct validation of the Dutch version of the impact of event scale. Psychological assessment. 2004; 16: 16.

48. Bloss CS, Wineinger NE, Darst BF, Schork NJ and Topol EJ. Impact of direct-to-consumer genomic 
Chapter 6 | Stages of behavioural change after DTC-GT disease risk profiling: study protocol

testing at long term follow-up. Journal of medical genetics. 2013: jmedgenet-2012-101207.

49. van der Zee KI, Sanderman R, Heyink JW and de Haes H. Psychometric qualities of the RAND 36-Item Health Survey 1.0: a multidimensional measure of general health status. International journal of behavioral medicine. 1996; 3: 104-22.

50. van der Zee KI and Sanderman R. Het meten van de algemene gezondheidstoestand met de RAND-36. Een handleiding[Measuring general health status with the RAND-36 Users manual] Groningen Northern Center of Health Care Research, The Netherlands. 1993.

51. Wallston KA, Wallston BS and DeVellis R. Development of the multidimensional health locus of control (MHLC) scales. Health Education \& Behavior. 1978; 6: 160-70.

52. Halfens $\mathrm{R}$ and Philipsen $\mathrm{H}$. Een gezondheidsspecifieke beheersingsorientatieschaal validiteit en betrouwbaarheid van de MHLC. T Soc Gezondheidsz. 1988; 66: 399-403.

53. Kaufman DJ, Bollinger JM, Dvoskin RL and Scott JA. Risky business: risk perception and the use of medical services among customers of DTC personal genetic testing. Journal of genetic counseling. 2012; 21: 413-22.

54. Carere DA, Kraft P, Kaphingst KA, Roberts JS, Green RC and Group PGS. Consumers report lower confidence in their genetics knowledge following direct-to-consumer personal genomic testing. Genetics in Medicine. 2015.

55. Teeuw B, Schwarzer R and Jerusalem M. Dutch adaptation of the general self-efficacy scale. 1994. URL: http://userpage fu-berlin de/- health/dutch htm. 2012.

56. Bode C, Thoolen B and Ridder d. Het meten van proactieve copingvaardigheden. PSEG. 2008; 36: 81-91.

57. Strijk JE, Wendel-Vos GCW, Picavet HSJ, Hofstetter H and Hildebrandt VH. Wat is vitaliteit en hoe is het te meten? Tijdschrift voor gezondheidswetenschappen. 2015; 93: 32-40.

58. Hoerger M, Quirk SW and Weed NC. Development and validation of the Delaying Gratification Inventory. Psychological assessment. 2011; 23: 725.

59. Goldberg LR. The development of markers for the Big-Five factor structure. Psychological assessment. 1992; 4: 26.

60. Bloss CS, Schork NJ and Topol EJ. Effect of direct-to-consumer genomewide profiling to assess disease risk. New England Journal of Medicine. 2011; 364: 524-34.

61. Skirton H, Jackson L, Goldsmith L and O'Connor A. Are health professionals ready for direct-toconsumer genetic and genomic testing? Personalized Medicine. 2013; 10: 673-82.

62. Lautenbach DM, Christensen KD, Sparks JA and Green RC. Communicating genetic risk information for common disorders in the era of genomic medicine. Annual review of genomics and human genetics. 2012; 14: 491-513.

63. Jaspers MWM. A comparison of usability methods for testing interactive health technologies: methodological aspects and empirical evidence. International journal of medical informatics. 2009; 78: 340-53. 


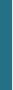


Non-communicable chronic diseases are one of the greatest threats of our society nowadays. One of the key causes, or perhaps the key cause, is our poor lifestyle. Despite many efforts, and successes, to improve our lifestyles, our behaviour still requires attention. This thesis has been a journey to explore a behaviour change from the perspective of genetic testing for disease risk.

The aim of this thesis was to elucidate some of the deficits of the personal genetic testing offered direct-to-consumer (DTC) and propose an alternative concept to address these deficits: Health Potential. In chapter 2 we explored predictors of acceptability and consideration in the Dutch general public. Chapter 3 laid the foundation for better disease risk estimation by integrating genetic, lifestyle and environmental factors of disease risk. Then in chapter $\mathbf{4}$ we explored important benefits and harms of DTC genetic testing (DTC-GT): behaviour change, negative psychological effects and medical consumption. As a first step to improve behaviour change, chapter $\mathbf{5}$ is the foundation for inclusion of concrete lifestyle advice to the genetic test results. Based on these findings we have developed a new disease prevention service: Health Potential. In chapter $\mathbf{6}$ we present the study protocol in which the effectiveness of the Health Potential service to change behaviour will be studied.

In this last chapter we will take a step back and look at the findings of this thesis in the light of other research and practice. In addition, we will briefly look at this thesis from an ethical perspective.

\section{Main findings and the relation to other research and practice}

\section{Striving for informed decision-making to undergo testing}

DTC-GT has been available on the market for over a decade and increasing numbers of people, on average around a third of Western populations, are now aware of its existence. ${ }^{1-4}$ In the near future, increasing numbers of people will be faced with a decision of whether or not to pursue genetic testing. Considering the nature of genetic testing and the possible implications of the results, it is essential that individuals are stimulated and supported to ensure this decision is an informed decision. For this, two elements are required: the decision must be (1) based on relevant information, and (2) in line with personal values. However, DTC-GT websites mostly overemphasise benefits of testing, and require a high level of literacy from the reader, thereby making it difficult for the consumer to obtain balanced information required for a well-considered decision.

Our results in chapter $2^{5}$ showed that having lower how-to knowledge (knowledge on 
understanding the DTC-GT results) was associated with greater consideration and intention to undergo testing, thereby possibly violating the first element. However, decision seemed to be in line with personal values, element 2 , as greater attitude was associated with greater acceptability, consideration and intention. These findings advocate provision of relevant information, thereby providing individuals with a complete and objective overview of all risks, benefits and implications of undergoing testing.

The results from this chapter describe factors associated with increased acceptability and consideration of undergoing DTC-GT. This information can be used in the development and distribution of information on the topic. With regard to informational content, for example, we found that lower how-to knowledge (knowledge on how to interpret the results) predicted consideration and intention, which therefore gives rise to including information on how test results would be interpreted in order to ensure that those who eventually pursue testing would be more likely to understand the obtained results. Selection of distribution channels used can also be guided by our findings. For example, the finding that innovativeness and younger age were significant predictors could suggest using social media channels over radio shows or printed newspapers.

\section{Weighing the pros and cons of undergoing DTC-GT}

$D T C-G T$ has the potential to initiate behaviour change

In chapter $4^{6}$, we performed a meta-analysis to shed light on the potential of DTC-GT to change lifestyle behaviour. Results show that DTC-GT has the potential to change behaviour: for example, $16 \%$ of participants had improved their diet, $12 \%$ exercised more, and $19 \%$ of smokers had quit smoking. Particularly the effect on quitting smoking is of interest with regard to a more accurate estimation of the effect DTC-GT may have on behaviour change. Namely, this estimate was restricted to smokers only, who all needed to quit. In contrast, the participants evaluated for dietary change, were likely not all in need of any or a lot of change, thereby underestimating the potential effect. Long-term follow-up is likely to reduce the number of people with (maintained) behaviour change, although studies examining long-term behaviour change are scarce. ${ }^{7}$

A larger effect was seen for information-seeking behaviour: 36\% of participants had sought further information after DTC-GT. This may indicate that participants did not yet reach the stage of actual behaviour change, but got stuck in preparatory stages of change in which they were weighing the advantages and disadvantages of changing. ${ }^{8}$ Together, these results suggest that DTC-GT has potential to serve as a lifestyle intervention, but additional support may be necessary to firstly move from informationseeking to actual change, and certainly if maintenance of change is to be pursued. 


\section{Worries about undue anxiety and distress but this does not seem grounded}

Opponents of DTC-GT have expressed concerns related to increased anxiety in response to genetic testing for common chronic disease risk. However, our research in chapter 4 found no increased anxiety, distress and worry as a result of testing, and any increased levels found faded with time. ${ }^{6}$ Looking closer, perceived control over the disease was associated with lower anxiety and distress. ${ }^{9}$ Whether size of the genetic risk reported was of influence is still unclear, ${ }^{9,10}$ and gender, ethnicity or education did not predict positive or negative psychological responses to testing. ${ }^{11}$

Further critical evaluation is recommended however, as the effects on the population as a whole may be mitigated through a self-selection process, where individuals who are prone to distress may be less likely to buy DNA tests. An exploratory study found that psychologically sensitive cases were significantly more likely to express concerns pre-testing, ${ }^{12}$ which could imply that they are also less likely to undergo testing. Finally, levels of regret of undergoing testing were low, which could further indicate the presence of self-selection. ${ }^{6,13-15}$ Future research may shed light on this hypothesis.

\section{An increased burden on the health care system seems limited}

Thoughts are that genetic testing offered without the interference of a health care professional (HCP) leads to an unnecessary and increased burden on the health care system. One argument in favour of involving a HCP before ordering a test is that he or she could serve as a gatekeeper and decides for or against testing for a particular individual to avoid unnecessary tests being performed. However, if HCPs do not critically evaluate the tests in light of their client's wishes, it may only lead to a false sense of reassurance in the client that the test is useful and appropriate for them. ${ }^{16}$ Therefore, if a HCP is to serve as a gateway to such a test, it is essential that they are trained and stimulated to critically reflect accordingly.

In addition, genetic test results for a large number of common chronic diseases are likely to identify several increased risks in every tested individual. These individuals may visit their HCP in order to help them evaluate the extent to which they are at risk and demand follow-up testing. Results from our meta-analysis (chapter $4^{6}$ ) show that approximately a third of tested individuals had shared their results with at least one $\mathrm{HCP}$ and that $7 \%$ had additional follow-up testing or screening. However, the number of people who shared their results may not necessarily reflect additional appointments with a physician. Namely, Kaufman et al. (2012) ${ }^{17}$ found that the longer people had had access to their results, the more likely they were to have shared it with their HCP. This is likely due to the fact that over time they would have had more opportunities for routine visits during which results could have been shared. In line with this, a longitudinal study 
specifically examining the effect on the health care system found that there was no effect of DTC-GT on the numbers and frequency of HCP visits, or the percentage of people with lab tests or procedures. ${ }^{18}$

Together the effects may not seem too substantial, but a previous study found that in case of (additional) follow-up care through regular medical provision, this may lead to downstream costs of between $\$ 40$ and $\$ 20,604$ per tested individual (estimation from 2009). ${ }^{19}$ One factor that increased downstream costs was referral to genetic specialists by primary care providers to assist answering patient's questions. This again calls for DTCGT companies to provide (1) clear communication of results and (2) designated trained specialists offering primary assistance and counselling for their clients. In addition, future research may further elucidate what the effect is of sharing results with HCPs, and what the added (unnecessary) medical costs are of DTC-GT. When this is known, it will be easier to intervene to avoid needless downstream costs.

In conclusion, research shows that although effects seem limited at this moment, there is a risk of creating unnecessary downstream medical costs and DTC-GT companies should be held primarily responsible to prevent this to their best ability.

\section{Addressing issues with DTC-GT}

More accurate prediction by integrating lifestyle and environmental factors into disease risk estimation

Disease risks are not determined by genetic factors alone. Therefore, it is too simplistic to base disease risk estimation solely on genetic factors, and lifestyle and environmental factors should be included too. ${ }^{20}$ Chapter 3 is a foundation for such an integration, using COPD as an example, providing an overview of all known genetic, lifestyle and environmental/occupational risk factors as studied in meta-analyses. ${ }^{21}$ This study identified 149 different genetic mutations associated with the risk of developing COPD. In addition, 7 lifestyle/environmental factors were identified, showing significant associations with risk estimates ranging from 0.45 to 9.50 . Not surprisingly, particularly smoking, both active and passive, contributed greatly to the risk of developing COPD, with smokers having a 3.5 times increased risk. Fortunately, most of the identified lifestyle/environmental factors are also modifiable to a lesser or greater degree, leading us to the next sub-chapter of improving actionability of the results.

\section{Improving actionability of the results}

Preventability of disease, or the opportunity to take action, is an important requisite for behaviour change. In addition, it may prevent anxiety or distress, as previous research has identified that increased perceived control, or the ability to control the disease 
through lifestyle changes, led to lower levels of anxiety and/or distress. ${ }^{9}$ Together, this calls for concrete lifestyle advice on how disease risk can be managed.

To this end, an initial requirement is that modifiable risk factors of these diseases are identified. As an example for one disease, we aimed to summarise all available evidence for modifiable risk factors associated with bladder cancer in chapter $5 .{ }^{22}$ To obtain estimates at the highest level of evidence, only meta-analyses were considered and were appropriate meta-meta-analyses were performed. This way, we identified 17 lifestylerelated and occupational risk factors of bladder cancer that were modifiable to a greater or lesser degree. In addition, we estimated that amongst individuals with non-optimal lifestyle behaviours (including low fruit and vegetable intake, high processed meat intake, currently smoking and low physical activity levels) $81.8 \%$ of cases could be prevented if these behaviours were eliminated. Knowledge such as presented in chapter 5 can be used to make disease risk information actionable by laying the foundation for concrete and scientifically substantiatened lifestyle advice.

Adding to actionability is another infrequently proposed but possibly very relevant value of genetic testing: the opportunity to prioritise lifestyle change. Lifestyle advice often is contradicting and people may get confused as to what is the healthiest behaviour. A well-known example is that of alcohol consumption. While low (but not none) alcohol intake lowers the risk of type 2 diabetes and stroke, ${ }^{23,24}$ alcohol intake increases the risk of breast cancer amongst others. ${ }^{25}$ As a result of this contrast, it is imaginable that people might get confused and frustrated, eventually discrediting and ignoring the advice or choose to do what already works best for them. However, by presenting disease risk estimations for multiple diseases, the client will have the information needed to prioritise their behaviour change and deal with conflicting advice. This way, lifestyle advice can be compared and weighed, tackling those risks first that needs most urgent attention, making them feel more confident that they are doing the right thing. It may still occur that advice is conflicting, but it will be easier to balance which advice is followed by taking the overall personal disease risk into account. Although some people may be able to evaluate this trade-off by themselves, others may need assistance. This, and other aforementioned issues, leads us to the next subchapter: added value of involvement of a HCP.

\section{Involvement of a health care professional}

Involving a HCP in the genetic testing process addresses several issues of DTC-GT. Firstly, involvement in the pre-test phase can stimulate clients to think about the results they will receive and improve considered decision-making with regard to testing. Several organisations have developed guidelines for pre-test information that should 
be reported on the website of DTC-GT. However, a recent study evaluated the pretest information DTC-GT companies reported and found that no single company adhered to all guidelines. They concluded that minimal requirements should be set by governmental bodies in order for DTC-GT to be used ethically and responsibly. ${ }^{26} \mathrm{In}$ addition, a previous study found that most DTC-GT consumers did not consider the fact that they may receive unwanted information, when considering undergoing testing, such as unexpectedly learning about risks for conditions one had not thought about. ${ }^{15}$ (S. Roberts, personal communication, March 26, 2018) In the current format, it is easy to consent online and move straight to testing, without so much as considering any of the impact of testing. ${ }^{27}$ If a knowledgeable person, a trained HCP, were involved, informed consideration of undergoing testing can be stimulated.

Secondly, involvement of a HCP may prevent or diminish misinterpretation of results. Although research into the comprehension of DTC-GT results has shown that in general most people interpreted risk estimates correctly, ${ }^{14,17,28,29}$ this does not necessarily reflect interpretation of results in the real-life setting. For example, in one study, participants were asked to indicate whether a fictitious person was at an increased or decreased risk of disease from multiple-choice answers; most correctly did so. ${ }^{17}$ However, when faced with own results it is likely that, even if one is able to objectively discern increased and decreased risks, one's personal health context as well as experiences with family and/or friends contribute to the subjective interpretation of the results, which could result in both under- and overvaluation of the actual risk. ${ }^{30}$ Therefore, it should be kept in mind that it is not self-evident that results are understood and interpreted correctly.

To start at the basis, it is initially essential that the results of the report be displayed in a comprehensible fashion so that they are self-explanatory. This will benefit both clients and any non-geneticist HCP who are requested to aid in interpreting the results. ${ }^{31}$ In the past years, several publications have addressed the design of genetic testing reports, providing suggestions and examples for clearer communication. ${ }^{31-33}$ In addition, in an attempt to regulate communication of results from DTC-GT for health-related purposes, several organisations have published statements and guidelines on the reporting of results, including the following items: results should be described in a way that is easy to understand and use; value of the results should not be overstated and it should be made clear that tests should not be used for diagnostic purposes or treatment decisions; customers should be made aware of non-genetic factors that contribute to the risk; data from scientific literature should be converted to estimates relevant for the consumer, such as relative and lifetime/absolute risks with specification of the type of risks; citing scientific information on which the test and results are based; all at an appropriate reading-level. ${ }^{20,34-36}$ Nevertheless, these guidelines are fairly vague (for example, when is a 
report "easy to understand and use"?) and each result report format will need evaluation to test adherence to these guidelines. In order to facilitate adherence to such guidelines, guidance, and more concrete information and examples could be provided. In addition, future research should focus on formats and content of information delivery from DTCGT, to attain the primary goal: stimulating behaviour change.

In addition, (genetic) counselling may be required or desirable for some groups. Previous studies found that comprehension was related to factors such as numeracy, genetic knowledge, ethnicity, educational level and age. ${ }^{14,17,28,29}$ In the true DTC-GT format the flow of information is unidirectional and it is unlikely to be noticed if the client has not understood the results correctly. In addition, a client may not always realise they do not fully understand or find it too much hassle to check whether they understood correctly. Involvement of a HCP in the delivery of results will lead to more interaction between information provider and receiver. The client will have the opportunity to ask any remaining questions and the HCP will be able to gauge whether he/she feels the message came across correctly.

Although a genetic counsellor could arguably be the most qualified candidate for such a role, they too would need training to accommodate the shift from mostly reactive singlegene diagnostic testing to pro-active multiplex testing focused at disease prevention. ${ }^{37}$

At this moment, several DTC-GT companies offer optional genetic counselling, but research has shown that this is infrequently taken up. ${ }^{38}$ Reasons for this were explored, revealing that those clients who did not utilise the service did not perceive a need as they already felt to understand their results. However, among those who did use the service, about half indicated that it had improved their understanding of the results and genetics in general. Therefore, it could be desirable to establish contact with a HCP prior to receiving results, thereby stimulating future (informal) contact. Naturally, it is essential that this HCP be adequately trained in interpretation and communication of risk information.

If an initial contact is established, the HCP can additionally function as an initiator, facilitator and catalyst for behaviour change and maintenance. Namely, he or she can assist the client in identifying areas that require greatest or most urgent change, and balancing potentially conflicting advice. Together they can develop a plan of action and maintenance strategies, in which the HCP may assist in other sources of help, such as other health professionals or tools. In addition, initial steps will have been made to establish a long-term relationship for behaviour change support. 


\section{Integrating these findings into a new service: Health Potential}

We have proposed Health Potential (www.healthpotential.eu) as an answer to several of the above mentioned issues. In short, Health Potential is a personal informational service on $20+$ common chronic diseases, providing its clients with the most up-todate and comprehensive disease risk estimates, including personalised lifestyle advice. This is achieved by integration of lifestyle, environmental and genetic factors in disease risk prediction, and all estimates are based on findings from (meta-)meta-analyses only. To encourage behaviour change and aid in understanding of the results, a HCP, which we will refer to as the Health Potential coach, will assist the client through the entire process, from pre-testing to post-testing and if desired, beyond. In addition, the result report contains concrete relevant personalised lifestyle advice to further facilitate behaviour change. This service not only holds value for the individual, but also for different stakeholders such as HCPs, investors and entrepreneurs, employers and insurance companies, society as a whole, and regulatory bodies, as will be elaborated upon in the valorisation addendum of chapter 8 .

With the introduction of our HCP-led genetic testing, I will henceforth use the more general term of personal genetic testing (PGT), encompassing both DTC and HCP-led delivery models.

\section{The Health Potential trial: the Leef!-study}

In order to evaluate this new disease prevention service, the Health Potential trial, nicknamed the Leef!-study, has been developed (chapter $6^{39}$ ).

The primary goal of the Health Potential service is to initiate and maintain behaviour change. Therefore, the primary aim of the trial is to study the effect of the Health Potential service on change in lifestyle behaviour. The secondary aims are (1) to study the effect of the Health Potential service on determinants of behaviour change, (2) to study the effect of additional lifestyle counselling on behaviour change and determinants thereof, and (3) to describe characteristics of the Health Potential customer.

In short, the design of the trial is as follows. The study consists of two integrated designs, part A and part B. Part A is a two-armed trial aimed to study the effectiveness of Health Potential service in achieving behaviour change and maintenance. Participants are recruited from local prevention clinics, and are not randomised but in stead reflect actual distribution of customers purchasing the Health Potential service with a general health check, or a general health check only. Part B is a two-armed trial aimed to study 
the effect of four additional lifestyle counselling sessions over a period of three months, on behaviour change and maintenance (secondary aim 2). Participants are randomised to receive either additional counselling or no additional support. Not randomising participants in part A will allow us to study the characteristics of the Health Potential customer (secondary aim 3). In addition, the setting will stay close to the user population that will be encountered in the real life setting. Thereby, the results of the study will more accurately reflect behaviour change when a service like Health Potential will be implemented.

\section{Ethical implications of this research}

Genetic testing for disease-related purposes is not free from ethical discussion, by far. In this section, relevant findings and topics from this thesis will be placed in a brief ethical perspective, using the four principles of bioethics as a guideline: autonomy, beneficence, non-maleficence and justice. ${ }^{40}$

One of the most important principles in the debate around PGT is one of autonomy. Autonomy refers to individuals being able to act intentionally, with understanding and without controlling influences. ${ }^{40}$ Proponents of PGT argue that individuals should be able to make their own decision whether or not to obtain information on their genome, which is often referred to as a right-to-know. ${ }^{41}$ Thus, in contrast to the traditional diagnostic genetic testing in the medical setting, it should be possible to obtain a genetic test without the restrictions of a physician required to grant the test. Similarly, nor should it be regulatory bodies dictating whether or not one may obtain a genetic test. However, instead of genetic testing being a right, it should rather be looked at as a 'freedom-to-know': individuals should be able to get a genetic test unless there is a sound reason to prevent them from doing so. ${ }^{42}$

This is where first of all the principle of non-maleficence comes in, essentially meaning: 'do no harm'. By allowing genetic testing, are we indeed not doing harm? Firstly, results from chapter 4 suggest that we do not seem to do harm with regard to psychological effects such as anxiety and/or distress. ${ }^{6}$ Secondly, at this moment the burden on the health care system seems limited but there is a risk of high downstream costs. ${ }^{6,19}$ If the latter does occur, PGT could result in a significant burden on society, particularly if this is not offset by other health benefits.

This leads us to the principle of beneficence. It has repeatedly been argued that PGT has little clinical utility, meaning in this case that it is unlikely for the test to improve 
medical decision-making. ${ }^{43}$ Interestingly however, individuals tend to perceived greater validity and utility of tests if they were offered through their physician, ${ }^{44}$ whereas HCPs perceived the utility of the tests as low and did not expect it to influence their care. ${ }^{45}$ However, one should question whether clinical utility should be the aim of genetic tests aimed at identifying increased disease risks. Namely, it should be a matter of whether or not PGT has preventive utility: does it lead to prevention of disease? Utility in that sense will be achieved through induction of behaviour change, including healthier lifestyles but also (self-)screening behaviours such as breast exams. In chapter $4^{6}$ we found that behaviour change after PGT offered DTC was no more than 20\%, with almost no effects on the long term. Nevertheless, PGT certainly holds potential to initiate behaviour change, serving as a cue to action, but it is likely that additional support is necessary to actually achieve change and particularly maintenance. This hypothesis will be tested in our trial, which was presented in chapter $6 .^{39}$

In addition, beneficence can be considered in lights other than clinical or preventive value. Previous users have mentioned reasons for undergoing a genetic test, including; curiosity and entertainment, ${ }^{46,47}$ participating in research and contributing to science, ${ }^{47-49}$ and among earliest adopters also the desire to be on the vanguard of embracing new technologies. ${ }^{50}$ Turrini et al. $2016^{51}$ provide an interesting narrative of different (additional) utilities of PGT. They argue that genetic information does not harbour intrinsic utility, but this is created by what man makes of it.

Finally, people undergoing testing for the value of entertainment may further explain that the majority of tested individuals do not improve their lifestyle behaviour. Namely, if people intend to undergo testing purely for the purpose of entertainment or out of curiosity, this is much less likely to lead to behaviour change, compared to those individuals who actively seek health-related information with the purpose of improving their health. In that sense, I expect future Health Potential clients to be more likely to pursue testing for the purpose of health compared to clients of DTC-GT services due to the extent of involvement that is required for Health Potential (e.g. visiting the HCP before obtaining the test, awaiting a meeting with the HCP to have results explained). Therefore the effect on behaviour change after undergoing Health Potential may already be greater (chapter $6^{39}$ ).

The fourth principle of justice is relevant to the extent that genetic testing is not available to low-income groups and thereby contributing to health disparities. Despite the fact that genetic testing has been steadily reducing in price in recent years, cost is still an important argument why individuals do not pursue testing. ${ }^{4}$ If genetic testing for common disease risk will prove to result in health benefits, the relatively high cost of 
testing may lead to an increased health gap between rich and poor, particularly when genetic testing will become more popular.

\section{Methodological considerations}

The focus of this chapter will be on difficulties we encountered in research on PGT.

\section{Risk of erroneous disease risk estimations}

Errors in disease risk estimation can occur at two stages: (1) during genotyping, i.e. analytical validity and (2) during translation of genotype to disease risks. Analytical validity is ensured through several regulations that pose criteria to ensure analytical validity, such as certification according to the Clinical Laboratory Improvement Amendments (CLIA) standards in the USA. Nevertheless, worries have been expressed of reduced vigilance of the laboratory as most genetic tests show no serious abnormalities, and the risk of mix-up between samples due to the high volume of samples handled. ${ }^{52}$ Research into this topic has shown that particularly the latter resulted in different results obtained from different testing companies. This was found to be the consequence of different mutations selected to include in the prediction model, reference groups that were selected, and different formulas used for risk calculation. ${ }^{53,54}$

\section{Actual consumers, research participants or hypothetical testing}

Studies on the topic of PGT have used different participant groups, which may each have different implications for the results. First of all, most studies lack an appropriate control group, thereby making it difficult to discern whether these effects are the result of undergoing testing or occurring naturally. Secondly, recruiting participants who undergo testing as part of a research setting may not reflect the actual group of people undergoing PGT and therefore behaviour change, psychological effects and sharing behaviour may be different. Thirdly, for studies using hypothetical scenarios reliability of results may be influenced by the difficulty of having to imagine effects of undergoing testing, particularly if one has not even seriously considered testing. This is the case, for example, for the survey by Bansback et al. (2012) $)^{55}$ in which participants were asked how they anticipated how results would influence their behaviour, if they were to, hypothetically, receive theirs. In addition, if respondents hear of the concept for the first time during the study, as was the case for the majority of our respondents in chapter 2, they likely had not yet given the matter sufficient thought and therefore their responses are likely to reflect instinctive reactions rather than well-considered decisions, which may change after learning more about the topic. ${ }^{56}$ To overcome issues of an inadequate control group in our trial, we will include individuals undergoing a general health check 
without genetic testing as a control group (chapter $\left.6^{39}\right)$.

\section{Lack of comparable outcome measures in PGT research}

Most studies on the effects of PGT use very heterogeneous outcome measures, which are difficult to compare. This was an important constraint in chapter $4^{6}$, where the studied effects (behaviour change, psychological effects and sharing behaviour) lacked comparable outcomes that could be combined in meta-analysis, and analyses were restricted to describing percentages of participants with some form of change. The outcomes for psychological effects were even too heterogeneous to allow any metaanalysis. Nevertheless, our results are the best summary estimates currently possible and provide relevant information, provided that the limitations of the findings are kept in mind. In addition, we were unable to examine of the size of the effect in meta-analyses. These issues point out the relevance of comparable measurement methods to study the effects of personal genetic testing.

\section{Meta-research}

Chapters 3 and 5 are typical examples of meta-research approaches. Meta-research is a mostly recent and upcoming field (although one of the first meta-meta-analyses was already published in $1979^{57}$ ), and can be considered to be "research of research". In the basis, our studies consisted of a systematic review of meta-analyses, which carries some inherent strengths and limitations.

Firstly, a great strength of focusing on meta-analyses instead of the underlying primary studies is that it lifts the level of evidence of our risk estimates to a higher level. This way, it was possible to achieve the necessary greater statistical power to study factors with relatively weak associations or when mostly studied in small study populations.

Secondly, results from meta-analysis are more generalizable to a larger population compared to estimates from observational or interventional studies. For disease risk prediction in a diverse population, such as with population screenings, this is a benefit as results will then on average be most applicable. However, if one aims to identify a disease risk in a specific individual, estimates from sub-group analyses may be more applicable, such as stratified by gender, ethnicity or even smoking status. Unfortunately, not all meta-analyses included such stratified analyses.

Thirdly, the level of publication bias can be studied. However, publication bias may, if present, also posit a threat to reliability of the results. In our studies, publication bias could be present at two levels: (1) primary studies with non-significant results are not published and thereby not included in the meta-analyses, leading to biased estimates, 
and (2) non-significant meta-analyses may not be published, resulting in not being identified by us. With regard to the first mentioned level, most studies we included examined publication bias and mostly found no significant bias. At the second level, in the (not unlikely) event that indeed non-significant meta-analyses remain unpublished, not identifying these studies will have little impact on our conclusion of which factors are associated with the studied disease if we assume the true association they found is indeed null and are not published for that reason. Therefore, publication bias at any of the two levels is likely to only have minimal impact on our conclusions. Adding to that, we ourselves may have missed studies for inclusion due to restrictions of the search engines with regard to length of the search string. Although we paid great attention to achieve simplification without missing relevant publications, we may still have missed some publications.

Fourthly, one of the greatest limitations of meta-analyses is that one is dependent on the analyses performed in the original studies. Evaluating, for example, the effects of study design or smoking status is not possible if the underlying studies do not report this. In turn, we cannot evaluate such detail if the meta-analyses themselves do not perform such analyses. An interesting solution is by performing a pooled analysis, in which, like in a meta-analysis, data from multiple studies is combined but at individual patient data (IPD) level rather than at an aggregate data (AD) level. IPD meta-analyses provide results with more detail and reliability and often include data from unpublished studies. ${ }^{58}$ Despite the latter, these studies are often limited by data made available to them, which in most cases is not complete. ${ }^{59}$ Thereby, IPD meta-analyses are still very prone to publication-related biases. However, as it is increasingly encouraged that patient-level data is made available for further research, we can expect a rise in IPD meta-analyses, hopefully without such biases.

Fifthly, reliability of our results depends largely on the quality of the underlying studies: the meta-analyses and their included primary studies. In chapter 3, we used the AMSTAR quality checklist to evaluate the quality of the included meta-analyses. We found that scores were poor for the majority of included meta-analyses. What the impact is on reliability of our findings is difficult to tell. Part of the low summary scores of AMSTAR may be explained by a matter of not reporting. For example, one of the AMSTAR items requires that an a-priori design is provided. Almost none of our publications mentioned this, which could be due to either not having one or simply not reporting that they had one. This leads us to the relevance of having reporting checklists for meta-research. Recently, such a checklist for overviews of systematic reviews was published, which appears to be appropriate for overviews of meta-analyses too. ${ }^{60}$ Although the focus of this checklist is on balanced reporting of benefits and harms from interventions, most items 
can apply to other meta-research, such as our studies, too. Nevertheless, an adaptation of such a checklist for the purpose of observational research would be helpful. Now, a next step would be to develop quality checklists for meta-research, which to the best of our knowledge currently does not exist.

Finally, in a more practical sense, this approach allows us to study a large number of diseases and risk factors in a reasonable amount of time, while maintaining high quality of evidence.

We supplemented our systematic review of meta-analyses with meta-meta-analyses where possible and appropriate. A meta-meta-analysis is essentially a meta-analysis of metaanalyses, also called a second-order meta-analysis. This allows the researcher to synthesise a large amount of data without having to access the primary studies. Combining metaanalytic estimates can lead to more comprehensive estimates, again increasing power over first-order meta-analysis. However, when combining meta-analytic estimates it should be kept in mind that any degree of overlap between included studies would lead to false homogeneity of results. Therefore, we decided to only deem meta-meta-analyses appropriate if no more than $50 \%$ of the studies of the smaller publication (i.e. the metaanalysis with fewest primary publications included) overlapped with those of the largest population (i.e. the most comprehensive meta-analysis).

\section{Epidemiological limitations}

Epidemiological research provides the foundation for disease risk estimations. Firstly, only relatively recently have researchers started to identify genetic mutations involved in development of multiplex diseases. Although a large chunk of the so-called low-hanging fruits will now have been picked, it is almost certain that in the coming years many more genetic factors will be identified, which will make disease prediction increasingly accurate. In that regard, one could argue that it is therefore too early to implement disease risk estimation practices in the general population, outside of the research setting. However, I would rather like to argue instead that it is inevitable that such services will be continued to be offered on the commercial market and therefore research into prediction models, delivery of results, and effects of the services are of paramount importance. One primary action in that sense too is for PGT companies to stay on top of the evidence and update disease risk prediction models with emergence of new scientific evidence.

Secondly, interactive effects between risk factors are likely to be at play but are currently understudied and are therefore insufficiently addressed in disease risk prediction. As such, gene-environment interactions are important to consider. For example, in chapter 
$3^{21}$ we identified associations between mutations in genes involved in the detoxification of polycyclic aromatic hydrocarbons (PAHs), such as EPHX1. Caucasian individuals with slower versions of the by the EPHX1 gene produced enzyme had an increased risk of developing COPD. ${ }^{61}$ As PAHs are one of the toxic compounds found in tobacco smoke, having a slow version of the detoxifying enzyme would be even more harmful for smokers and requires more urgent action. Although the topic is getting increasing attention, current evidence is mostly based on relatively small studies, and study and reporting methods could be improved. ${ }^{62}$ In addition, interaction between lifestyle and environmental factors are equally important for disease risk prediction. One example would be the synergistic effect between asbestos and smoking on lung cancer risk. ${ }^{63}$ Similarly, studying dietary patterns rather than single food items may provide more insight. When such interaction effects, particularly interactions with or between nongenetic factors, will be integrated in disease risk predictions, more personalised and relevant lifestyle advice can be given.

\section{Final remarks/conclusion}

In conclusion, PGT certainly has potential to fulfil multiple values; for consumers, HCPs and society alike. Nevertheless, we must maintain a critical view to ensure such services are implemented and utilised in a way that promotes benefits and fulfils individual values, while averting negative consequences. This thesis provided several directions as to how this can be approached. 


\section{References}

1 Cherkas, L. F., Harris, J. M., Levinson, E., Spector, T. D. \& Prainsack, B. A survey of UK public interest in internet-based personal genome testing. PloS one 5, e13473 (2010).

2 Ortiz, A. P. et al. Awareness of direct-to-consumer genetic tests and use of genetic tests among Puerto Rican adults, 2009. Preventing chronic disease 8, A110 (2011).

3 Agurs-Collins, T. et al. Public Awareness of Direct-to-Consumer Genetic Tests: Findings from the 2013 US Health Information National Trends Survey. Journal of Cancer Education 30, 799-807, doi:10.1007/s13187-014-0784-x (2015).

4 Mavroidopoulou, V., Xera, E. \& Mollaki, V. Awareness, attitudes and perspectives of direct-toconsumer genetic testing in Greece: a survey of potential consumers. Journal of human genetics 60 , 515-523, doi:10.1038/jhg.2015.58 (2015).

5 Stewart, K. F. J. et al. Factors associated with acceptability, consideration and intention of uptake of direct-to-consumer genetic testing: a survey study. (Submitted).

6 Stewart, K. F. J., Wesselius, A., Schreurs, M. A. C., Schols, A. \& Zeegers, M. P. Behavioural changes, sharing behaviour and psychological responses after receiving direct-to-consumer genetic test results: a systematic review and meta-analysis. J Community Genet 9, 1-18, doi:10.1007/ s12687-017-0310-z (2018).

7 Bloss, C. S., Wineinger, N. E., Darst, B. F., Schork, N. J. \& Topol, E. J. Impact of direct-toconsumer genomic testing at long term follow-up. Journal of medical genetics 50, 393-400, doi:10.1136/jmedgenet-2012-101207 (2013).

8 Prochaska, J. O. \& Velicer, W. F. The transtheoretical model of health behavior change. American journal of health promotion 12, 38-48 (1997).

9 Boeldt, D. L., Schork, N. J., Topol, E. J. \& Bloss, C. S. Influence of individual differences in disease perception on consumer response to direct-to-consumer genomic testing. Clinical genetics 87, 225-232, doi:10.1111/cge.12419 (2015).

10 Haga, S. B. et al. Impact of delivery models on understanding genomic risk for type 2 diabetes. Public health genomics 17, 95-104 (2014).

11 O'Neill, S. C., Tercyak, K. P., Baytop, C., Hensley Alford, S. \& McBride, C. M. A new approach to assessing affect and the emotional implications of personal genomic testing for common disease risk. Public health genomics 18, 104-112, doi:10.1159/000370101 (2015).

12 Broady, K. M., Ormond, K. E., Topol, E. J., Schork, N. J. \& Bloss, C. S. Predictors of adverse psychological experiences surrounding genome-wide profiling for disease risk. J Community Genet, doi:10.1007/s12687-017-0339-z (2017).

13 Carere, D. A., Kraft, P., Kaphingst, K. A., Roberts, J. S. \& Green, R. C. Consumers report lower confidence in their genetics knowledge following direct-to-consumer personal genomic testing. Genetics in Medicine 18, 65-72 (2016).

14 Kaphingst, K. A. et al. Patients' understanding of and responses to multiplex genetic susceptibility test results. Genetics in medicine : official journal of the American College of Medical Genetics 14, 681-687, doi:10.1038/gim.2012.22 (2012).

15 Roberts, J. S. et al. Direct-to-Consumer Genetic Testing: User Motivations, Decision Making, and Perceived Utility of Results. Public health genomics 20, 36-45, doi:10.1159/000455006 (2017).

16 McGowan, M. L., Fishman, J. R., Settersten, R. A., Jr., Lambrix, M. A. \& Juengst, E. T. Gatekeepers or intermediaries? The role of clinicians in commercial genomic testing. PloS one 9, e108484, doi:10.1371/journal.pone.0108484 (2014).

17 Kaufman, D. J., Bollinger, J. M., Dvoskin, R. L. \& Scott, J. A. Risky business: risk perception and the use of medical services among customers of DTC personal genetic testing. Journal of genetic counseling 21, 413-422, doi:10.1007/s10897-012-9483-0 (2012).

18 Reid, R. J. et al. Association between health-service use and multiplex genetic testing. Genetics in medicine : official journal of the American College of Medical Genetics 14, 852-859, doi:10.1038/ gim.2012.52 (2012). 
19 Giovanni, M. A. et al. Health-care referrals from direct-to-consumer genetic testing. Genetic testing and molecular biomarkers 14, 817-819, doi:10.1089/gtmb.2010.0051 (2010).

20 Human Genetics Commission. A Common Framework of Principles for direct-to-consumer genetic testing services. (2010). <https://web.archive.org/web/20111114144949/http://www.hgc. gov.uk/UploadDocs/DocPub/Document/Principles consultation final.pdf>.

21 Stewart, K. F. J., Mount, S. W., Wesselius, A., Zeegers, M. P. \& Schols, A. M. W. J. Genetic, lifestyle, and environmental factors associated with development of chronic obstructive pulmonary disease. (Submitted).

22 Stewart, K. F., Al-Zalabani, A. H., Wesselius, A., Schols, A. M. \& Zeegers, M. P. Modifiable risk factors for the prevention of bladder cancer: a systematic review of meta-analyses. European journal of epidemiology 31, 811-851 (2016).

23 Li, X.-H., Yu, F.-f., Zhou, Y.-H. \& He, J. Association between alcohol consumption and the risk of incident type 2 diabetes: a systematic review and dose-response meta-analysis. The American journal of clinical nutrition 103, 818-829 (2016).

24 Zhang, C. et al. Alcohol intake and risk of stroke: a dose-response meta-analysis of prospective studies. International journal of cardiology 174, 669-677 (2014).

25 Shield, K. D., Soerjomataram, I. \& Rehm, J. Alcohol use and breast cancer: a critical review. Alcoholism: Clinical and Experimental Research 40, 1166-1181 (2016).

26 Hall, J. A., Gertz, R., Amato, J. \& Pagliari, C. Transparency of genetic testing services for 'health, wellness and lifestyle': analysis of online prepurchase information for UK consumers. European journal of human genetics : EJHG 25, 908-917, doi:10.1038/ejhg.2017.75 (2017).

27 Gilbert, M. et al. Click yes to consent: Acceptability of incorporating informed consent into an internet-based testing program for sexually transmitted and blood-borne infections. International journal of medical informatics 105, 38-48, doi:10.1016/j.ijmedinf.2017.05.020 (2017).

Ostergren, J. E. et al. How Well Do Customers of Direct-to-Consumer Personal Genomic Testing Services Comprehend Genetic Test Results? Findings from the Impact of Personal Genomics Study for the PGen Study Group. Public health genomics 18, 216-224 (2015). McGrath, S. P., Coleman, J., Najjar, L., Fruhling, A. \& Bastola, D. R. Comprehension and DataSharing Behavior of Direct-To-Consumer Genetic Test Customers. Public health genomics 19, 116-124, doi:10.1159/000444477 (2016).

30 Miron-Shatz, T., Hanoch, Y., Katz, B. A., Doniger, G. M. \& Ozanne, E. M. Willingness to test for BRCA1/2 in high risk women: Influenced by risk perception and family experience, rather than by objective or subjective numeracy? Judgment and Decision Making 10, 386-399 (2015).

Haga, S. B. et al. Developing patient-friendly genetic and genomic test reports: formats to promote patient engagement and understanding. Genome medicine 6, 58 (2014).

32 Shaer, O. et al. Informing the Design of Direct-to-Consumer Interactive Personal Genomics Reports. Journal of medical Internet research 17, E146-U186, doi:10.2196/jmir.4415 (2015).

33 Lautenbach, D. M., Christensen, K. D., Sparks, J. A. \& Green, R. C. Communicating genetic risk information for common disorders in the era of genomic medicine. Annual review of genomics and human genetics 14, 491-513 (2013).

34 American College of Medical Genetics and Genomics. Direct-to-consumer genetic testing: a revised position statement of the American College of Medical Genetics and Genomics. Genetics in medicine : official journal of the American College of Medical Genetics 18, 207-208, doi:10.1038/ $\operatorname{gim} .2015 .190$ (2016).

35 FDA. (2017).

36 Hudson, K., Javitt, G., Burke, W. \& Byers, P. ASHG statement on direct-to-consumer genetic testing in the United States. Obstetrics and gynecology 110, 1392-1395 (2007).

37 Mills, R. \& Haga, S. B. Genomic Counseling: Next Generation Counseling. Journal of genetic counseling 23, 689-692, doi:10.1007/s10897-013-9641-z (2014).

38 Darst, B. F., Madlensky, L., Schork, N. J., Topol, E. J. \& Bloss, C. S. Perceptions of genetic counseling services in direct-to-consumer personal genomic testing. Clinical genetics 84, 335-339, 
doi:10.1111/cge.12166 (2013).

39 Stewart, K. F. J., Wesselius, A., Schols, A. M. W. J. \& Zeegers, M. P. Stages of behavioural change after direct-to-consumer disease risk profiling: study protocol of two integrated controlled pragmatic trials. Trials (In Press).

40 Beauchamp, T. L. \& Childress, J. F. Principles of Biomedical Ethics. (2009).

41 Howard, H. C. \& Borry, P. Is there a doctor in the house?: The presence of physicians in the directto-consumer genetic testing context. Journal of Community Genetics 3, 105-112 (2012).

42 Martinez Otero, J. M. "Better Not to Know?": Justifiable Limits on the Right to Information in the Realm of DTC Genetic Testing. An Analysis of the European and Spanish Legal Framework. European journal of health law 24, 175-197 (2017).

43 Grosse, S. D. \& Khoury, M. J. What is the clinical utility of genetic testing? Genetics in Medicine 8, 448 (2006).

44 Critchley, C., Nicol, D., Otlowski, M. \& Chalmers, D. Public reaction to direct-to-consumer online genetic tests: Comparing attitudes, trust and intentions across commercial and conventional providers. Public understanding of science (Bristol, England) 24, 731-750, doi:10.1177/0963662513519937 (2015).

45 Vassy, J. L. et al. 'Someday it will be the norm': physician perspectives on the utility of genome sequencing for patient care in the MedSeq Project. Personalized Medicine 12, 23-32, doi:10.2217/ pme.14.68 (2015).

46 Vayena, E., Gourna, E., Streuli, J., Hafen, E. \& Prainsack, B. Experiences of early users of directto-consumer genomics in Switzerland: an exploratory study. Public health genomics 15, 352-362, doi:10.1159/000343792 (2012).

47 Mahlmann, L., Rocke, C., Brand, A., Hafen, E. \& Vayena, E. Attitudes towards personal genomics among older Swiss adults: An exploratory study. Applied and Translational Genomics 8, 9-15 (2016).

48 Vayena, E., Ineichen, C., Stoupka, E. \& Hafen, E. Playing a part in research? University students' attitudes to direct-to-consumer genomics. Public health genomics 17, 158-168, doi:10.1159/000360257 (2014).

49 Su, Y., Howard, H. C. \& Borry, P. Users' motivations to purchase direct-to-consumer genomewide testing: An exploratory study of personal stories. Journal of Community Genetics 2, 135-146 (2011).

50 McGowan, M. L., Fishman, J. R. \& Lambrix, M. A. Personal genomics and individual identities: Motivations and moral imperatives of early users. New genetics and society 29, 261-290 (2010).

51 Turrini, M. \& Prainsack, B. Beyond clinical utility: The multiple values of DTC genetics. Applied and Translational Genomics 8, 4-8 (2016).

52 Sunderman, S. F. The Need for Regulation of Direct-to-Consumer Genetic Testing in the United States: Assessing and Applying the German Policy Model. Wash. U. Global Stud. L. Rev. 12, 357 (2013).

53 Kalf, R. R. et al. Variations in predicted risks in personal genome testing for common complex diseases. Genetics in medicine : official journal of the American College of Medical Genetics 16, 85-91, doi:10.1038/gim.2013.80 (2014).

54 Swan, M. Multigenic condition risk assessment in direct-to-consumer genomic services. Genetics in Medicine 12, 279-288 (2010).

55 Bansback, N., Sizto, S., Guh, D. \& Anis, A. H. The effect of direct-to-consumer genetic tests on anticipated affect and health-seeking behaviors: a pilot survey. Genetic testing and molecular biomarkers 16, 1165-1171, doi:10.1089/gtmb.2012.0074 (2012).

56 Kaphingst, K. A. et al. Consumers' use of web-based information and their decisions about multiplex genetic susceptibility testing. Journal of medical Internet research 12, e41, doi:10.2196/ jmir.1587 (2010).

57 Kazrin, A., Durac, J. \& Agteros, T. Meta-meta analysis: A new method for evaluating therapy outcome. Behaviour Research and Therapy (1979). 
58 Stewart, L. A. \& Tierney, J. F. To IPD or not to IPD? Advantages and disadvantages of systematic reviews using individual patient data. Evaluation \& the health professions 25, 76-97 (2002).

59 Ahmed, I., Sutton, A. J. \& Riley, R. D. Assessment of publication bias, selection bias, and unavailable data in meta-analyses using individual participant data: a database survey. BMJ (Clinical research ed.) 344, d7762 (2012).

60 Bougioukas, K. I., Liakos, A., Tsapas, A., Ntzani, E. \& Haidich, A.-B. Preferred reporting items for overviews of systematic reviews including harms checklist: a pilot tool to be used for balanced reporting of benefits and harms. Journal of clinical epidemiology 93, 9-24 (2018).

$61 \mathrm{Li}, \mathrm{H}$., Fu, W. P. \& Hong, Z. H. Microsomal epoxide hydrolase gene polymorphisms and risk of chronic obstructive pulmonary disease: A comprehensive meta-analysis. Oncology letters 5, 1022 1030 (2013).

62 Simonds, N. I. et al. Review of the Gene-Environment Interaction Literature in Cancer: What Do We Know? Genetic epidemiology 40, 356-365 (2016).

63 Ngamwong, Y. et al. Additive synergism between asbestos and smoking in lung cancer risk: A systematic review and meta-analysis. PloS one 10, e0135798 (2015). 
Chapter 7 | General Discussion 


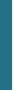


As the world is facing a challenging epidemic of non-communicable diseases, such as cancer, diabetes type 2, and cardiovascular diseases, primary prevention is becoming increasingly paramount. Important risk factors for these diseases include smoking, poor diet and physical inactivity. These risk factors are to a large extent modifiable and therefore pose targets for prevention.

At the same time, personalised health care is undergoing rapid developments. One interesting emerging field in that light is that of personalised genetic testing (PGT) for disease risks. These tests aim to estimate an individual's risk of developing a certain disease based on their genetic profile. These services are mostly offered online without interference of a health care professional ( $\mathrm{HCP})$, thereby referred to as direct-toconsumer genetic testing (DTC-GT). It has been hypothesised that if one learns to be at an increased risk of disease, they may be triggered to change their health behaviour. For example, a smoking individual may consider quitting when learning of an increased risk of lung cancer. In that way, PGT may be an important approach in the pursuit of preventing chronic diseases.

In this thesis I address different issues regarding PGT; from pre-test informed decisionmaking to the need for post-test counselling in achieving behaviour change. I conclude by proposing a new PGT service, Health Potential, as an alternative approach to address these issues.

Chapter 1 starts with a general introduction into the topic, introducing the issues surrounding PGT that are addressed in this thesis.

Chapter 2 starts at the pre-testing phase, where we studied characteristics of the expected consumer population of DTC-GT in order to provide assistance in the development of pre-test information to enhance informed decision-making. More particular, we studied factors associated with acceptability of, and consideration (some time in the future) and intention ( $<1$ year) of undergoing DTC-GT. Multiple correlates were identified, but of particular interest is the finding that lower knowledge of genetic principles was associated with greater acceptability, and lower understanding of DTC-GT results was associated with greater consideration and intention. As for an informed decision, it is necessary that decisions are based on complete and relevant information, our results may indicate that informed decision-making may be hampered. The identified characteristics can be used in development and distribution of pre-test information, in order to help consumers make a truly informed decision.

In chapter 3, I move on to address the limitation of basing disease-risk predictions on 
genetic factors only, by reporting an overview of all genetic, lifestyle and environmental risk factors, using COPD as an example. This study gives an overview of the currently available evidence of lifestyle, environmental, and genetic factors associated with the development of COPD. We identified 274 genetic variants, of which $74 \%(n=204)$ showed a significant association with ORs ranging from 0.17-3.33. In addition, we identified nine different lifestyle and environmental factors, for which a significant association was found for eight, with ORs ranging from 0.45-9.50. In the light of personalised prevention, these results can be combined to make disease risk prediction more accurate.

Then, chapter 4 reports the results of a systematic review with meta-analysis into different effects of DTC-GT, for which there has been a lot of discussion among academics and regulatory bodies: behaviour change, adverse psychological effects and post-test medical consumption. We found that overall $24 \%$ had a positive lifestyle change. More specifically, $16 \%$ and $12 \%$ had improved dietary and exercise practices respectively, whereas $19 \%$ quit smoking. In addition, $7 \%$ of participants had subsequent preventive checks. Finally $33 \%$ had shared their results with any health care professional and 50\% had shared it with family and/or friends. Although meta-analysis was not possible for psychological responses, results showed that anxiety, distress and worry were low or absent and that the effect faded with time. These results indicate that DTC-GT has potential to change behaviour, without great risk of adverse psychological responses, but that attention must be paid to avoid unnecessary downstream costs as a result of testing.

In chapter 5 I move on with improving actionability of results by including lifestyle advice based on modifiable risk factors for disease. I do so using bladder cancer as an example, in a systematic review with meta-meta-analysis of modifiable risk factors of bladder cancer. We found a great number of modifiable risk factors that were significantly associated with the development of bladder cancer: current $(R R=3.14)$ or former $(R R=1.83)$ cigarette smoking, pipe $(R R=1.90)$ or cigar $(R R=2.30)$ smoking, antioxidant supplementation $(R R=1.52)$, obesity $(R R=1.10)$, higher physical activity levels $(R R=0.86)$, higher body levels of selenium $(R R=0.61)$ and vitamin $D(R R=0.75)$, and higher intakes of: processed meat $(R R=1.22)$, vitamin $A(R R=0.82)$, vitamin $E$ ( $R R=0.82)$, folate $(R R=0.84)$, fruit $(R R=0.77)$, vegetables $(R R=0.83)$, citrus fruit $(\mathrm{RR}=0.85)$, and cruciferous vegetables $(\mathrm{R} R=0.84)$. Finally, three occupations with the highest risk were tobacco workers $(R R=1.72)$, dye workers $(R R=1.58)$, and chimney sweeps $(\mathrm{RR}=1.53)$. In addition, we calculated a probability of causation, which can be interpreted as the percentage of exposed cases that are attributable to the exposure (e.g. the number of cases among smokers that are attributable to smoking). Probabilities of causation for individual factors ranged from $4 \%-68 \%$. We also calculated a combined 
probability of causation, combining 4 non-overlapping factors: total fruit and vegetable consumption, processed meat consumption, smoking, and physical activity. This showed that up to $81.8 \%$ of the bladder cancer cases, among those with non-optimal lifestyle behaviours, could be prevented through lifestyle modifications. Together these results provide starting points for actionable behaviour change advice.

From here I continue with the service we developed, Health Potential. This service is a PGT service that estimates personal disease risk of $20+$ common chronic diseases, with both a genetic and lifestyle component of development. In contrast to DTC-GT, this service is obtained and delivered by a health care professional, who can (1) assist in pre-test decision-making to achieve an informed decision, (2) aid in interpreting of test results, and (3) offer (on-going) support to initiate and maintain behaviour change posttesting. Chapter 6 reports the study protocol for our Health Potential trial, in which we will test how effective our new Health Potential service is in changing behaviour and whether additional counselling is necessary to achieve and maintain behaviour change. The study consists of two integrated designs resulting in three study arms. Participants are clients of local prevention clinics, purchasing a personalised health check (PHC; intervention condition), consisting of Health Potential ${ }^{\bullet}$ and a general health check, or the general health check alone (GHC; control condition) (Part A). PHC participants will be randomised to receive four additional lifestyle counselling sessions over a period of three months (Part B). Results from this study can then provide valuable insights in possible ways forward in the field of personalised prevention.

Finally, chapter 7 contains the general discussion, placing the findings in light of other research, as well as from an ethical perspective.

To conclude, PGT certainly has potential to fulfill multiple values; for consumers, HCPs and society alike. Nevertheless, we must maintain a critical view to ensure such services are implemented and utilised in a way that promotes benefits and fulfils individual values, while averting negative consequences. This thesis provided several directions as to how this can be approached. 


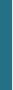




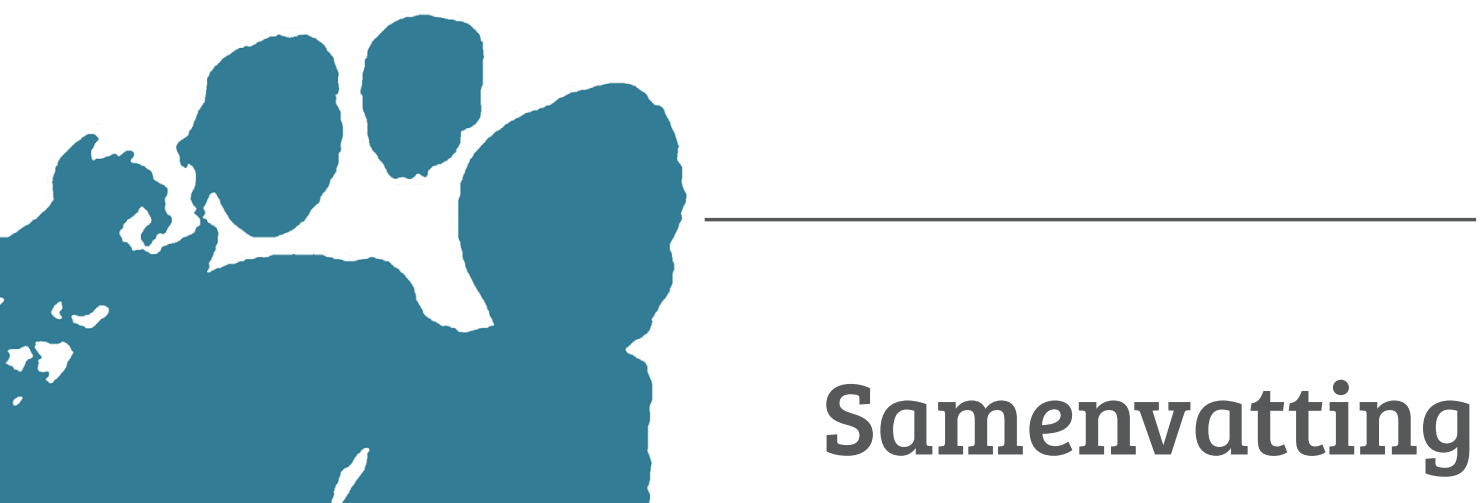

$x$

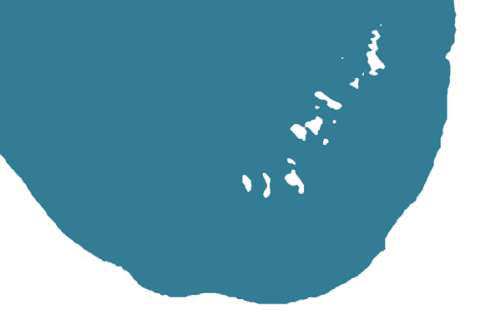


We kampen wereldwijd met een groeiende epidemie van niet-overdraagbare aandoeningen, zoals kanker, diabetes type 2 en hart- en vaatziekten. Om deze trend aan te pakken is primaire preventie van groots belang. Belangrijke risicofactoren voor deze ziekten zijn onder andere roken, slechte eetgewoonten en fysieke inactiviteit. Aangezien deze risicofactoren veranderbaar zijn, vormen ze belangrijke doelen zijn voor preventie.

Tegelijkertijd zijn er snelle ontwikkelingen in de gepersonaliseerde gezondheidszorg. Een interessant gebied dat volop in ontwikkeling is, is dat van persoonlijke genetische testen (PGT) voor ziekterisico. Met deze testen wordt het risico geschat op het ontwikkelen van een bepaalde ziekte op basis van iemands genetisch profiel. Deze diensten worden momenteel met name online aangeboden zonder dat hier een gezondheidsprofessional aan te pas komt. Ze worden dan ook direct-to-consumer genetische testen (DTC-GT) genoemd; testen die direct aan de consument worden aangeboden. Een van de ideeën achter deze testen is dat wanneer iemand leert dat hij een verhoogd risico heeft op het ontwikkelen van een bepaalde ziekte, hij of zij wordt aangespoord zijn of haar leefstijl te veranderen om zo het risico te verlagen. Zo kan bijvoorbeeld een roker overwegen te stoppen als hij leert dat hij een verhoogd risico heeft op het ontwikkelen van longkanker. Zo kan PGT bijdragen aan preventie van niet-overdraagbare chronische aandoeningen.

In deze thesis heb ik verschillende zaken rondom PGT onderzocht; van het maken van een weloverwogen beslissing voor het ondergaan van een genetische test (informed-decision making) tot de noodzaak van counseling na afloop van testen om zo gedragsverandering te bereiken. Ik sluit af met een nieuw-ontwikkelde PGT dienst, Health Potential, waarmee verschillende kwesties rond deze diensten worden aangepakt.

In Hoofdstuk 1 begin ik met een algemene inleiding van het onderwerp, waarbij ik de verschillende kwesties rondom PGT die in deze thesis besproken worden introduceer.

Hoofdstuk 2 begint in de fase voorafgaand aan testen, waar we kenmerken van mogelijke toekomstige consumenten hebben onderzocht. Aan de hand van deze kenmerken kunnen informatie bronnen ontwikkeld worden, waarmee potentiele gebruikers tot een weloverwogen besluit kunnen komen om DTC-GT te ondergaan. In het bijzonder hebben we gekeken naar factoren die geassocieerd waren met acceptatie van DTC-GT, als ook het overwegen (ergens in de toekomst) en van plan zijn (in het komende jaar) om DTC-GT te ondergaan. Hier kwamen meerdere kenmerken uit naar voren, waaronder leeftijd en beoordeling van eigen gezondheid, maar wat met name interessant was, was dat mensen met minder basiskennis in de genetica DTC-GT vaker acceptabel vonden, en dat mensen die de resultaten van zo'n test minder goed begrepen vaker overwogen of van plan waren om zich te laten testen. Om tot een weloverwogen besluit te komen 
is het belangrijk dat deze keuze gebaseerd is op volledige en relevante informatie. Deze bevindingen betekenen daarom dat een weloverwogen besluit mogelijk beperkt wordt door onvoldoende kennis onder de Nederlandse bevolking. De kenmerken die in dit onderzoek naar voren zijn gekomen kunnen gebruikt worden in de ontwikkeling en verspreiding van informatie omtrent DTC-GT, om zo bij te dragen aan het tot stand brengen van een weloverwogen besluit om DTC-GT te ondergaan.

In hoofdstuk 3 staat integreren van verschillende typen risicofactoren centraal: genetische, leefstijl- en omgevingsfactoren. Hiervoor wordt de longziekte COPD als voorbeeld gebruikt. Deze studie geeft een uitgebreid overzicht van huidige bewijslast voor al deze factoren, welke geassocieerd zijn met het ontwikkelen van COPD. In totaal identificeerde we 274 genetische varianten, waarvan $74 \%(n=204)$ significant in verband stonden met COPD, en waarbij odds ratio's (ORs) varieerden van $0.17-3.33$. Daarnaast vonden we negen verschillende leefstijl- en omgevingsfactoren, waarvan acht significant geassocieerd waren met COPD, en waarbij ORs varieerden van $0.45-9.50$. In het kader van gepersonaliseerde preventie is het belangrijk dat deze verschillende typen factoren gecombineerd worden om zo het schatten van ziekterisico's nauwkeuriger te maken.

Vervolgens bespreek ik in hoofdstuk $\mathbf{4}$ de resultaten van een systematische review met meta-analyses naar de verschillende effecten van DTC-GT: gedragsverandering, psychologische effecten en extra medische aandacht (testen of bezoeken aan een arts) na testen. Uit onze meta-analyses bleek dat $24 \%$ van DTC-GT gebruikers een positieve leefstijlverandering had. Als we naar specifieke gedragingen keken, bleek dat 16\% hun eetpatroon had veranderd, $12 \%$ meer was gaan bewegen en $19 \%$ van de rokers gestopt was met roken. Daarnaast bleek dat 7\% van DTC-GT gebruikers na de test preventieve controles had ondergaan. Tot slot had 33\% hun resultaten gedeeld met een gezondheidsprofessional en $50 \%$ met familie en/of vrienden. Voor de psychologische effecten was een meta-analyse helaas niet mogelijk, maar uit de individuele studies konden we concluderen dat er weinig tot geen sprake was van angst, zorgen of ongerustheid als gevolg van DTC-GT, en als dit wel zo was, dit niet blijvend was. Deze resultaten laten zien dat DTC-GT potentie laat zien om leefstijl te veranderen, zonder al te groot risico op negatieve psychologische effecten, maar dat er wel aandacht besteed moet worden om te voorkomen dat er onnodige kosten worden gemaakt als gevolg van extra medische testen na DTC-GT.

In hoofdstuk 5 ga ik verder met het verbeteren van bruikbaarheid van de resultaten door het toevoegen van leefstijl advies op basis van veranderbare risicofactoren voor een ziekte. Dit doe ik aan de hand van blaaskanker als voorbeeld, in een systematische review met meta-meta-analyse (een meta-analyse van meta-analyses) naar alle veranderbare 
risicofactoren voor deze ziekte. In deze studie identificeerden we een groot aantal veranderbare risicofactoren die significant in verband stonden met het ontwikkelen van blaaskanker: huidig $(R R=3.14)$ of voormalig $(R R=1.83)$ roken van sigaretten, pijp $(R R=1.90)$ of sigaar $(R R=2.30)$ roken, antioxidant supplementen $(R R=1.52)$, obesitas $(R R=1.10)$, hoger niveau van fysieke activiteit $(R R=0.86)$, hogere lichaamswaarden van selenium $(R R=0.61)$ en vitamine $D(R R=0.75)$, en hogere innames van: bewerkt vlees $(R R=1.22)$, vitamine $A(R R=0.82)$, vitamine $E(R R=0.82)$, folaat $(R R=0.84)$, fruit $(R R=0.77)$, groenten $(R R=0.83)$, citrusvruchten $(R R=0.85)$, en kruisbloemige groenten $(\mathrm{RR}=0.84)$. Daarnaast zijn de drie meest risicovolle beroepsgroepen: zij die werken met tabak $(R R=1.72)$ en kleurstof $(R R=1.58)$, en schoorsteenvegers $(R R=1.53)$. Tot slot berekenden we de probability of causation (vrij vertaald als waarschijnlijkheidspercentage), wat geïnterpreteerd kan worden als het percentage van blootgestelde ziektegevallen wat toegewezen kan worden aan die specifieke blootstelling (bijvoorbeeld het aantal rokers met blaaskanker waarbij roken verantwoordelijk was voor de ontwikkeling van de ziekte). De probability of causation varieerde van $4 \%$ tot $68 \%$ voor individuele factoren. We berekenden ook een gecombineerde probability of causation waarbij we totale groente en fruit inname, bewerkt vlees inname, roken en fysieke activiteit combineerden. Dit liet zien dat tot wel $81.8 \%$ van de blaaskanker gevallen, onder degene met een suboptimale leefstijl, voorkomen zouden kunnen worden via aanpassingen in hun leefstijl. Deze resultaten vormen een basis voor het toevoegen van bruikbare leefstijl adviezen bij de DTC-GT resultaten, uiteraard met als uiteindelijk doel ziektepreventie.

Hiervandaan ga ik verder met de dienst die we hebben ontwikkeld: Health Potential ${ }^{\circ}$. Deze dienst is een PGT dienst waarbij ziekterisico's worden geschat voor $20+$ verschillende veelvoorkomende chronische ziekten, waarbij zowel genetica als leefstijl bijdragen aan de ontwikkeling van de ziekte. In tegenstelling tot DTC-GT wordt deze dienst via een gezondheidsprofessional aangeboden. Hij of zij zal dan (1) begeleiden in het bereiken van een weloverwogen besluit om te gaan testen, (2) hulp bieden bij het interpreteren van de resultaten, en (3) (langdurige) hulp bieden na afloop van testen om gedragsverandering in gang te zetten en te behouden. Hoofdstuk $\mathbf{6}$ beschrijft een studie protocol voor onze Health Potential ${ }^{\circ}$ trial, waarin we zullen testen hoe effectief onze nieuw ontwikkelde dienst is in het veranderen van gedrag en of extra counseling nodig is om gedragsverandering te bereiken en vol te houden. De studie bestaat uit twee geïntegreerde onderzoeksopzetten (deel A en deel B) waarmee samen 3 studie-armen ontstaan. Deelnemers zijn klanten van lokale preventieklinieken, die een gepersonaliseerde gezondheidscheck kopen (de interventie conditie), bestaande uit Health Potential ${ }^{\circ}$ en een algemene gezondheidscheck, of enkel de algemene gezondheidscheck (controle conditie); deel A van de studie. Deelnemers in de interventie conditie worden daarnaast nog gerandomiseerd om vier extra leefstijl counseling sessies te ontvangen over een periode van drie maanden (deel 
B). Resultaten van deze studie zullen waardevolle inzichten geven voor de toekomstige aanpak van PGT.

Tot slot beschrijft Hoofdstuk 7 de algemene discussie, waarbij de bevindingen van deze thesis worden vergeleken met ander onderzoek. Ook wordt hier aandacht besteed vanuit het ethisch perspectief.

Concluderend kunnen we zeggen dat PGT zeker de potentie heeft om voor verschillende mensen verschillende waarden te vervullen; voor zowel consumenten, gezondheidsprofessionals als de maatschappij. Desondanks moeten we een kritische blik houden om er voor te zorgen dat zulke diensten zodanig geïmplementeerd en gebruikt worden dat ze vooral de voordelen stimuleren en individuele waarden vervullen, terwijl negatieve gevolgen afgewend worden. Dit proefschrift biedt verschillende aanknopingspunten voor hoe dit aangepakt kan worden. 


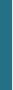




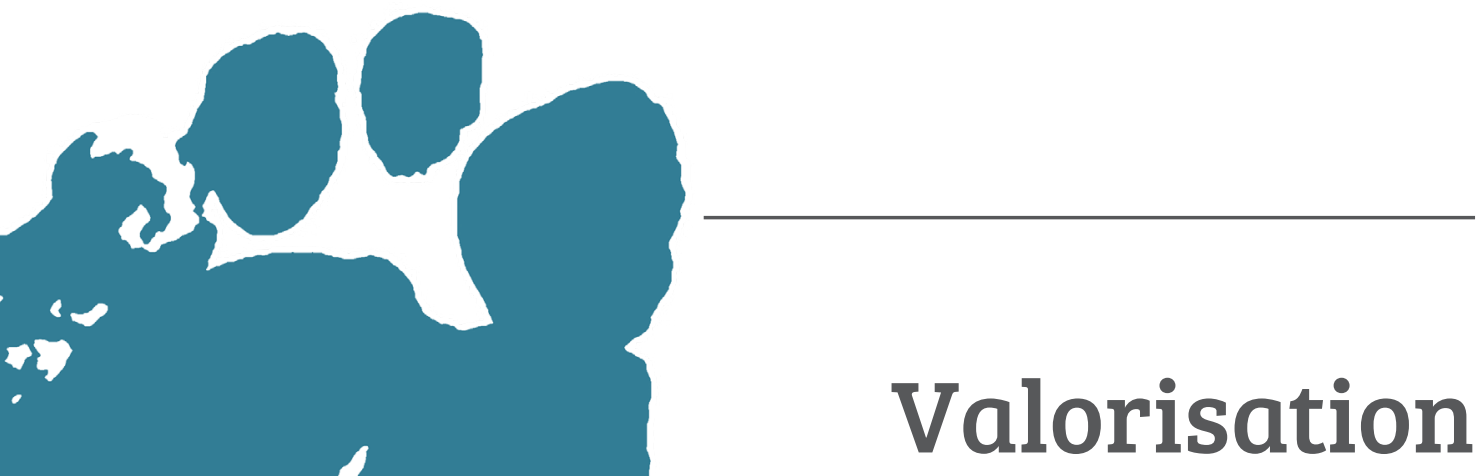

$x$

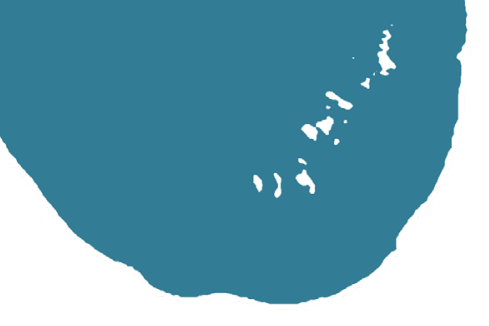


During the research for this thesis, many opportunities for valorisation of the acquired knowledge arose and some were actually seized. This chapter addresses these opportunities and more, and discusses societal relevance of the results of this thesis.

A majority of the work conducted for this thesis was dedicated to developing a reliable, valid, functional and practical personal disease risk testing service. This service, Health Potential, will soon be launched on the international market. We, as the Maastricht University Health Potential team, are based in a valorisation hotspot, with the proximity and opportunities of Brightlands/Maastricht Health Campus, several universities (e.g. Eindhoven, Ghent, Hasselt and Aachen), the EU region Meus-Rhine (Dutch: Euregio Maas-Rijn), and of course Maastricht University's 'Maastricht Valorisation Centre', providing an excellent accelerator for diffusion and diversification.

I will first discuss the relevance of the results of this thesis for different potential stakeholders. Then, because involvement of stakeholders in product development is essential for successful products, I will conclude with one example of how we involved end-users in development: usability testing of our online Health Potential platform.

\section{Relevance of this work for different stakeholders}

\section{The individual}

One of the primary and perhaps most obvious target populations is the individual enduser; the customer. These individuals are most likely to benefit from the Health Potential service directly, through acquiring information and starting points for behaviour change. In addition, it is likely fulfill other needs through the 'fun-factor' and satisfaction of curiosity. This may particularly be the case for the early adopters who might generally be more curious about the service in general compared to later adopters who might learn about it when searching for health-related information.

\section{The health care professional}

Health Potential is a source of personal health-related information, which can be used for different purposes including supporting health behaviour change. As such, it can be used and implemented in many different health-care settings and different types of health care professionals (HCPs; including dieticians, physiotherapists, weight loss coaches or even nurse practitioners) can use Health Potential concurrent to or merged with their own services. As we cannot expect every HCP stay up-to-date with all disease risk knowledge available, which expands at an astonishing rate, services like Health Potential offer them a means to stay on top of the evidence. Their role is then no longer to collect the information, but rather to help their patient or client to interpret it, place 
it in context of their personal situation, help them to translate it to their desired use, and finally support them in the maintenance of their lifestyle change.

In addition, results from chapter 4 provide suggestions as to who might need additional assistance to avoid adverse psychological effects, thereby making the service delivery as safe as possible.

\section{Entrepreneurs and investors}

Our convenient location with border-crossing interactions between Maastricht University, Hogeschool Zuyd, and local businesses and start-ups, offers an excellent environment for exploitation of opportunities. This contributes to easy contact with (other) entrepreneurs and investors, resulting in interesting partnerships and collaborations.

Health Potential as a product, as well as its methods, is an excellent basis for diversification, which can lead to new collaborations and initiatives. Currently, different products with a similar approach are being developed, both on our initiative as on request from other parties. These products are based on a similar method as Health Potential but address a different domain. One such example is Health Potential's sister product, Sport Potential, which assesses athletic potential based on one's genetic profile. Similar to the research presented in chapters 3 and 5, for Sport Potential we analysed the genetic mutations associated with different athletic characteristics in a similar manner as for Health Potential. ${ }^{1}$

In addition, chapter 2 , which provides information on predictors of interest in DTCGT, may be useful for this group too as this will help to identify their target population and thereby market their products more effectively and appropriately.

\section{Employers and insurance companies}

Both employers and insurance companies have a similar benefit from services as Health Potential: improving the health of their employees/clients to reduce their own costs. Namely, absenteeism and reduced productivity, which are associated with poor lifestyles ${ }^{2}$, is a major source of costs for employers. Similarly, health care or disability insurers would have to pay up less if people were healthier. As a result, increasing amounts of attention, time and money are being spent on promoting health of their employees and clients, in which (services like) Health Potential could play a role.

At this moment, first steps have been taken to cooperate with employers and insurance companies. For this, a lean version of Health Potential will be used, including non- 
genetic factors only, due to potential privacy and discriminatory issues related to the use of genetic data. Although we are still in the preparatory phases of this collaboration, prospects appear to be good.

\section{Society}

Value for society as a whole will primarily be achieved if Health Potential is effective in improving lifestyle and thereby improving future health. A healthier population will have increased production, decreased sick-leave associated costs, and on the long-term reduced health-care costs. However, the impact on society on the short-term is likely to be small, if any, as only a relatively small group of people will initially take up Health Potential and this group is likely to already be more health minded, thereby possibly not addressing the population most at need of lifestyle improvements. In addition, for behaviour change to be maintained optimally on the long term, thereby achieving observable benefits for society, a multidisciplinary approach with interventions at micro, meso and macro levels, is not just desirable but likely a necessity. Finally, for optimal benefits for society, it is a prerequisite that Health Potential does not lead to (unnecessary) downstream medical costs.

\section{Regulatory bodies}

Chapter 4 provides input on the balance of pros and cons in the discussion of allowing DTC-GT on the market. As such, it showed that DTC-GT does generally not lead to adverse psychological effects, has moderate impact on behaviour change, and may lead to downstream medical costs. These findings can be taken into consideration during drafting of regulations. In addition, chapter 2 provides input for information provision to the general population regarding DTC-GT. This information should ideally be provided by independent bodies and will enhance informed decision-making of undergoing testing.

\section{Involvement of end-users: the example of our online platform}

To enhance acceptance by and value for end-users, we aim to closely involve different stakeholders in development of our products. This sub-chapter describes the involvement of future coaches and clients in the development of our online platform, which is designed to assist both coaches and clients in monitoring the process of testing. Here, both parties can view the current status and respond to required actions. To develop this online application, we collaborated with Hello Sunshine (Heerlen, The Netherlands), and applied a user-centred design cycle, which consisted of multiple short sprints or iterations during which the product was continuously improved: a cyclic process of 
design, evaluation and redesign ${ }^{3,4}$. Our goal was to develop a platform that would be convenient and intuitive to use. After several initial cycles based on interaction between Health Potential researchers and developers, potential end-users (clients and coaches) and the application developers, a first version had been developed and was tested and improved in future users, combining different usability testing methods.

\section{User test 1: Heuristic evaluation}

\section{Methods}

The first version of the system was evaluated using heuristic evaluation, using Nielsen's heuristics (Table 1$)^{5}$. These heuristics are general principles that are common properties of usable systems.

To identify a variety of usability issues ${ }^{6}, 11$ evaluators were recruited including usability testing experts, technology experts, and application domain experts. All evaluators had great interest in applications and use of technology and used technology on a regular basis. Before testing, each evaluator was trained in Nielsen's heuristics and was reminded of Health Potential and the purpose of the system. They were asked to navigate through the application at their own pace, doing and clicking as they pleased, writing down their findings, both positive and negative.

All findings were collected and analysed for usability issues (defined as not being able to advance or being distracted or discouraged from a flaw in design or content of the application). At minimum, the critical issues were resolved before we continued with user testing of the next version of the application.

Table 1 Nielsen's 10 heuristics for user-friendly interface design

1. Visibility of system status

2. Match between system and the real world

3. User control and freedom

4. Consistency and standards

5. Error prevention

6. Recognition rather than recall

7. Flexibility and efficiency of use

8. Aesthetic and minimalist design

9. Help users recognise, diagnose, and recover from errors

10. Help and documentation

\section{Results}

The heuristic evaluation identified a total of 222 unique issues with the application. Issues 
mainly regarded difficulty navigating through the application and the questionnaire in particular. Evaluators mentioned that they missed a quick navigation option for the questionnaire, which then had to be navigated one question at the time, and that some icons for menu buttons were unclear. They suggested the use of a breadcrumb (i.e. a navigational element showing the current page in relation to previous pages visited or a certain hierarchy of pages) for easier orientation in and navigation through the application. In addition, they experienced confusion with validation of answers and skip logic within the questionnaire (e.g. skipping female-only questions for males). In addition, it was considered odd that notifications were not clickable, and ideally they should lead to a location the notification referred to. Finally, errors or ambiguities in textual content were identified, particularly within the questionnaire, and evaluators expressed a wish for additional information at certain locations.

\section{User test 2: eye tracking and 'thinking aloud method'}

\section{Methods}

We did two series of user testing: one with prospective coaches and one with prospective clients. For this, we used the concurrent think-aloud approach combined with eye tracking. This method involves encouraging the participants to verbalise their thoughts while navigating the application. At the same time, we used eye tracking to allows more objective evaluation of system design and the different interactions of a user with the system which can not easily be observed otherwise ${ }^{7}$. One can evaluate the amount of processing that the user applies through the time that he or she fixates on one design element ${ }^{8}$.

Each test was centred around a set of scenarios, describing the context behind why a user would be using the application, including intake, collecting of data and delivery of the results. The scenarios were shown to the participants on the screen to be read and additional instructions were given by the facilitator of the test during the execution of the test.

The sessions were video recorded and transcribed verbatim. These transcripts were then used to generate a list of usability issues, which were rated on severity and assigned the location and interface aspects, like was done during heuristic analysis.

\section{Results}

In total, four coaches and five clients participated. All coaches were physiotherapists or exercise scientists. Together they identified a total of 62 unique issues. One menu icon (the hamburger menu icon; figure 1) that had not been changed as it was thought to be a commonly used icon, still caused confusion. However, users quickly figured out what 
it meant and how to use it. In addition, the hover-effect, the technique that the design of an element changes when the mouse cursor is on that element and which was thought to give the impression that notifications were clickable, had been removed but this had apparently not resolved the issue. Finally, two coaches mentioned that although the design of the application looked functional, it could use a 'flashy layer'.

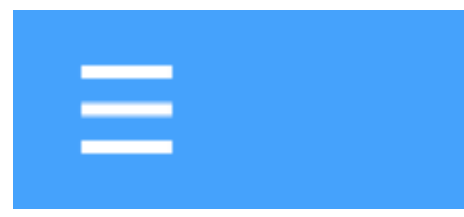

Figure 1 Hamburger menu icon

One of the most important areas of the application for the user is the timeline. This shows the user's progress through the entire Health Potential procedure, and is thought to help the users monitor and accelerate their testing process by showing which steps have been completed, what needs to be done or what the user is currently waiting for. Testing showed that all-but-one user interpreted the timeline correctly as multiple processes running parallel to each other (Figure 2) and it seemed to support their perception of the overview of the process.

\section{Continued evaluation}

After these two testing rounds, a new version of the application was developed, which was subsequently further improved through multiple informal evaluation rounds with the Health Potential team, development team and other relevant stakeholders. Right before the formal launch of Health Potential, the application, together with all other processes of Health Potential, will be tested as a whole in a pilot run with actual consumers. Naturally, it will remain an on-going process of development, continually improving and expanding the options and possibilities that we can offer. However, this cycle of design, evaluation and redesign nicely illustrates how such cycles could look. 


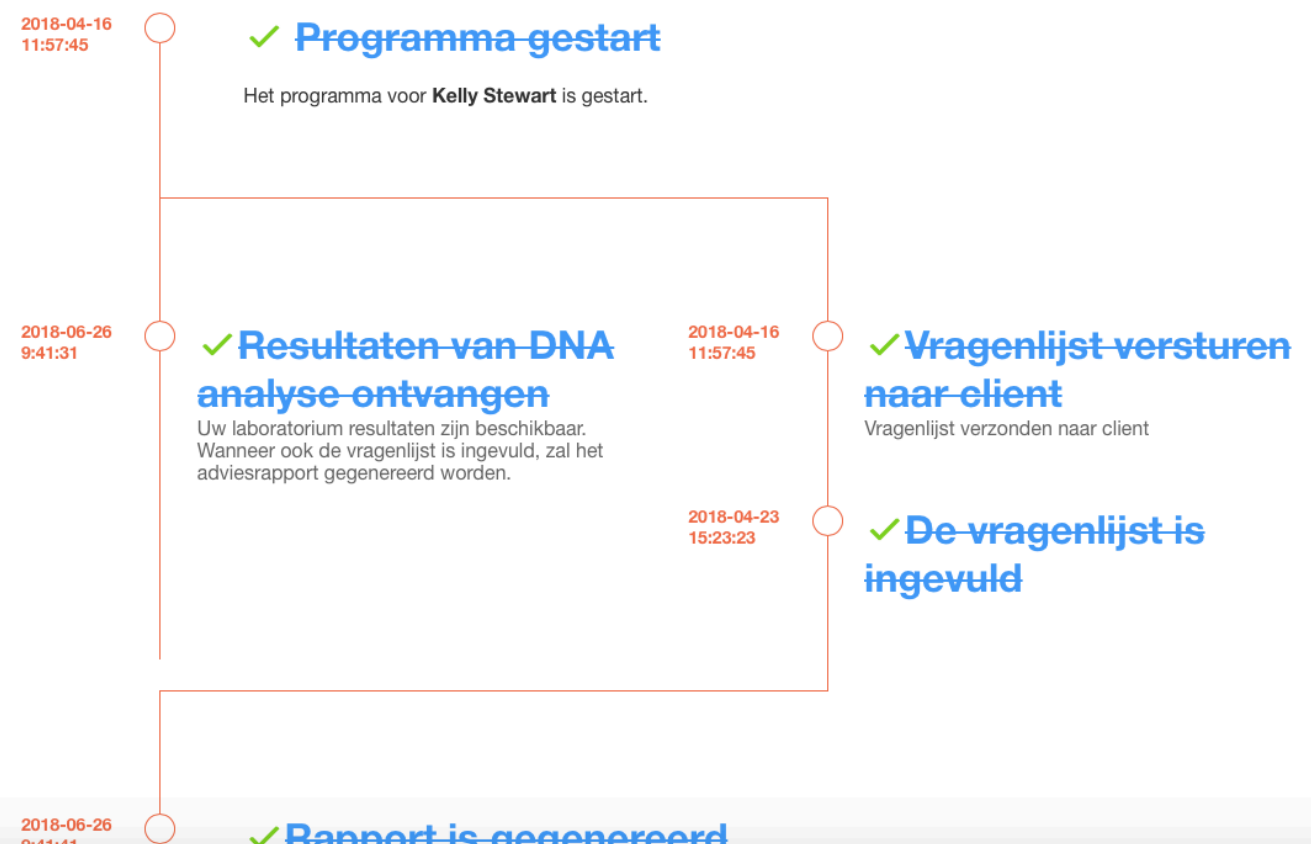

Figure 2 Screenshot of timeline with parallel running processes

In user testing greater focused was placed on the risk report than during heuristic evaluation. Issues in the report were mostly related to interpretation of the information therein. Although some layout changes were suggested that would reduce confusion, such as a change in the order of presentation of genetic and lifestyle factors, most of the issues required additional training, counselling or a more elaborate help function, which are now being developed. 


\section{References}

1. Weyerstraß J, Stewart K, Wesselius A, Zeegers M. Nine genetic polymorphisms associated with power athlete status-A Meta-Analysis. Journal of science and medicine in sport. 2018;21(2):213-20.

2. Fitzgerald S, Kirby A, Murphy A, Geaney F. Obesity, diet quality and absenteeism in a working population. Public health nutrition. 2016;19(18):3287-95.

3. Nielsen J. Usability engineering. Boston, MA: AP Professional; 1994.

4. Gulliksen J, Göransson B, Boivie I, Blomkvist S, Persson J, Cajander Å. Key principles for usercentred systems design. Behaviour and Information Technology. 2003;22(6):397-409.

5. Nielsen J. Heuristic evaluation. In: Nielsen J, and Mack, R.L., editor. Usability Inspection Methods. New York, NY: John Wiley \& Sons; 1994.

6. Hertzum M, Molich R, Jacobsen NE. What you get is what you see: revisiting the evaluator effect in usability tests. Behaviour \& Information Technology. 2014;33(2):144-62.

7. Poole A, Ball LJ. Eye tracking in HCI and usability research. Encyclopedia of human computer interaction. 2006;1:211-9.

8. Duchowski A. Eye tracking methodology: Theory and practice: Springer Science \& Business Media; 2007. 


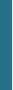


Haast ondenkbaar, maar mijn proefschrift is af. Ruim vier jaar verder; een bewogen vier jaar met veel ups en downs, die ik nu af mag sluiten met dit boekje. Natuurlijk ben ik veel mensen dank verschuldigd, zonder jullie had dit boekje er nu niet gelegen. (Behalve lieve Isla, zonder jou had het boekje er misschien wat eerder gelegen, maar dat is je vergeven)

Allereerst ben natuurlijk ontzettend veel dank verschuldigd aan mijn (co-)promotoren Anke, Maurice en Annemie.

Anke, ik ben zo blij geweest met jou als sparring partner de afgelopen jaren. We hebben menig 'probleem' samen opgelost, nieuwe ideeën uitgewerkt en ook op persoonlijk vlak kon ik bij je terecht.

Maurice, ondanks dat ik soms gek werd van je constant veranderende ideeën, plannen, nieuwe gadgets en apps, je hebt me zeker uit mijn veilige prehistorische-dinosaurus wereld gehaald! Ik heb genoten van het delen van onze interesse in sport en tuin.

Annemie, ook al hebben we elkaar niet vaak gesproken in de afgelopen tijd, je wist toch telkens een interessante draai aan mijn project te geven. Voor elke meeting was ik weer benieuwd wat er nu op ons pad ging komen. Een frisse wind!

Daarnaast ook hartelijk dank aan de leden van mijn beoordelingscommissie:

Prof. dr. S.A. Morré, Dr. S. Braun, Prof. dr. S.P.J. Kremers, Prof. Dr. H. Snieder, and Prof. Dr. D.M.R. Townend.

Great thanks to all my office buddies! Starting off with just a few, we are now a very large, interesting and diverse group. In particular, Nikos, thanks for reminding me to be "not so serioes Kelly!". I still think about our time in Florence with great joy. And of course Frits and Miriam, never shy of a gossip! It may not always have resulted in uninterrupted researching, but definitely brought lots of new ideas! And how about the great movie nights and dinners, walks, bbq's that we shared. Hopefully there are more to come!

We definitely shared a lot of moments these past years Sarah! Sharing manuscripts and maternity clothes, trying every of your baby bottles, and loads of silly messages. And eventually we even got this never-ending ginormous paper submission-ready. Woohooo!!

Hanneke, je hebt me zo vaak geholpen de afgelopen jaren! Toen we hoorden dat je weg ging dachten we dat we gedoemd waren. Gelukkig heb je een goede opvolger gevonden 
(Bedankt voor de hulp de laatste maanden, Erika! Heerlijk zo'n vrolijke wind op de afdeling) en zien we nog eens!

Liesbeth and Daša, it was a nice 'last-minute' experience writing my final paper of my thesis with you. I enjoyed looking at my topic from a different view.

Thank you, Abdulmohsen, for our collaboration on multiple papers now. I really enjoy working with you and admire your thoroughness and patience (and patience with me...). I enjoyed learning about your culture very much. I hope we can work on another paper again in the future!

Een dank aan mijn stagiaires en honours studenten. Ik hoop dat jullie minstens zo veel van mij geleerd hebben als ik van jullie.

Ik heb veel genoten en geleerd van mijn dagen bij Hello Sunshine, en vooral van Jeroen en Danny. Wat een gezellige boel mensen zijn jullie!

Melanie en François, ik heb veel geleerd van de usability testen die we samen uitgevoerd hebben, bedankt!

Randy, Cyriel en vooral Roelof bij Stofberg, ik heb veel plezier gehad in onze samenwerking in de afgelopen jaren!

We zien elkaar lang niet vaak genoeg, Leanne, Saskia en Romke, maar onze vriendschap blijft heel bijzonder. Zodra we samen zijn, is het alsof we elkaar pas nog zagen. Jullie hebben me door heel wat momenten gesleept de afgelopen jaren! Bijzonder dank voor Saskia, waar ik altijd met mijn vragen rondom onderzoek, publiceren en proefschriften terecht kan.

Kim, kun je je voorstellen dat we elkaar al kennen sinds we de leeftijd van onze ukkies hadden? Ik vind het heel fijn dat ik zo veel jeugdherinneringen met je kan delen. En hoeveel dingen we sindsdien gedeeld hebben, 'real-life' maar ook via de app. Trouwen, kindjes krijgen, gezondheidsproblemen... En nu allebei promotie ;).

Maartje en Rebecca, ik kan heerlijk zinloos roddelen met jullie, tripjes maken, wijntjes en biertjes drinken, eten en in het kader van deze thesis: spuien over werk. Ik heb de afgelopen jaren veel te vaak afgehaakt vanwege werk, zwangerschap, reisafstand en Isla, en jullie blijven begripvol. (Nog wel, haha!) 
Bedankt dat je me Londen door sleepte, Esther, toen ik daar op een congres was. Zonder jou had ik mijn vrije tijd daar misselijk en uitgeput in bed doorgebracht. (Ook al sliep ik alsnog tijdens Les Miserables)

Bianca, wat begon al die jaren geleden in erg vrij leven met heel veel craic, is nog altijd een fantastische vriendschap. Ik hoef geen goede buur als ik jou als verre vriend heb.

Maar een goede buur heb ik wel! Thomas, Marleen en grappige Ollie, fijn dat ik af en toe bij jullie kan komen spuien (en biertjes drinken en worstenbroodjes eten).

Mijn lieve Nederlandse familie Engbersen, jullie leven altijd mee! Ook al weten jullie volgens mij nog niet precies wat ik doe, dat doet er voor jullie niet toe. Bedankt voor de fijne achterban die jullie zijn.

And of course my all-over-the-world Stewart family. Particularly Gary, Carol, Sean, Amy and Callum, you're my safe haven in Ireland, which I haven't visited enough in the past four years, by far! Gary, you have been a great inspiration and motivation, and are never reluctant to throw in a few compliments to make me feel a little better about myself. Joe and Irene, I found it great that I could visit you whilst on a course in Newcastle!

Ellis en Robert, bedankt voor al jullie ondersteuning. Weer verhuizen? Geen probleem, jullie komen gewoon weer schilderen. (Dat is nu klaar, dat beloof ik) Ik waardeer en bewonder het ook enorm dat jullie het geen enkel punt vinden om Isla op te vangen, of het nu een geplande dag is of dat we om 7 uur's avonds uitgeput opbellen. Ik ga er vanuit dat de meeste hectiek nu achter de rug is. Bedankt dat jullie ons er doorheen hebben geholpen.

Papa en mama, jullie hebben onbeschrijflijk veel voor me gedaan. Nu Isla er is realiseer ik me pas hoe veel energie jullie al die jaren in me hebben gestoken. Jullie hebben onvoorwaardelijke liefde en aandacht gegeven en me helpen te vormen tot wie ik nu ben. De afgelopen jaren hebben jullie ons vaak ondersteund, op welke manier op dat moment dan ook mogelijk was. Jullie stappen écht spontaan in de auto om meer dan een uur te rijden om me een knuffel te komen geven.

Een jaar of 17 geleden hield jij een boomstam vast terwijl ik hem in stukjes probeerde te hakken. Lieve Thijs, als ik daar aan denk is het best een wonder dat we hier al die jaren later samen staan. Het was wel een eerste aanwijzing dat we een goed team zijn. We versterken elkaar op verschillende vlakken en weten inmiddels waar ieders krachten 
(en beperkingen) liggen. Ook met mijn werk heb je me vaak gesteund en stukken voor me gelezen. Je kan me heel goed motiveren en coachen, en ik vind het fijn dat ik met je kan sparren over mijn onderzoek!

$\mathrm{Nu} 9$ jaar samen heb je me meer dan eens (soms letterlijk) weer terug op mijn pootje(s) gezet, ook al viel je daardoor zelf weer om. Hopelijk breekt nu een periode van relatieve rust aan (of is dat ijdele hoop met ons?) en kunnen we genieten van ons huis, tuin, maar vooral ons gezinnetje. Want je blijkt toch echt wel een hoop papa-genen in je te hebben, ook al vond je soms zelf van niet. Ik kan ontzettend genieten wanneer jullie met z'n tweeën aan het stoeien en gek doen zijn.

En, je hebt me het grootste cadeau ooit gegeven. Lieve Isla, jij kan me de wereld om ons heen echt laten vergeten. Je gegiechel en gegrijns, eindeloze energie, stevige knuffels, natte kussen, en de vele nachtelijke uurtjes die we delen. Het waren een heftige laatste maanden, maar met hulp van papa, opa's en oma’s hebben we ons er doorheen geslagen. Ik kijk uit naar wat de toekomst voor ons in petto heeft. 


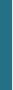




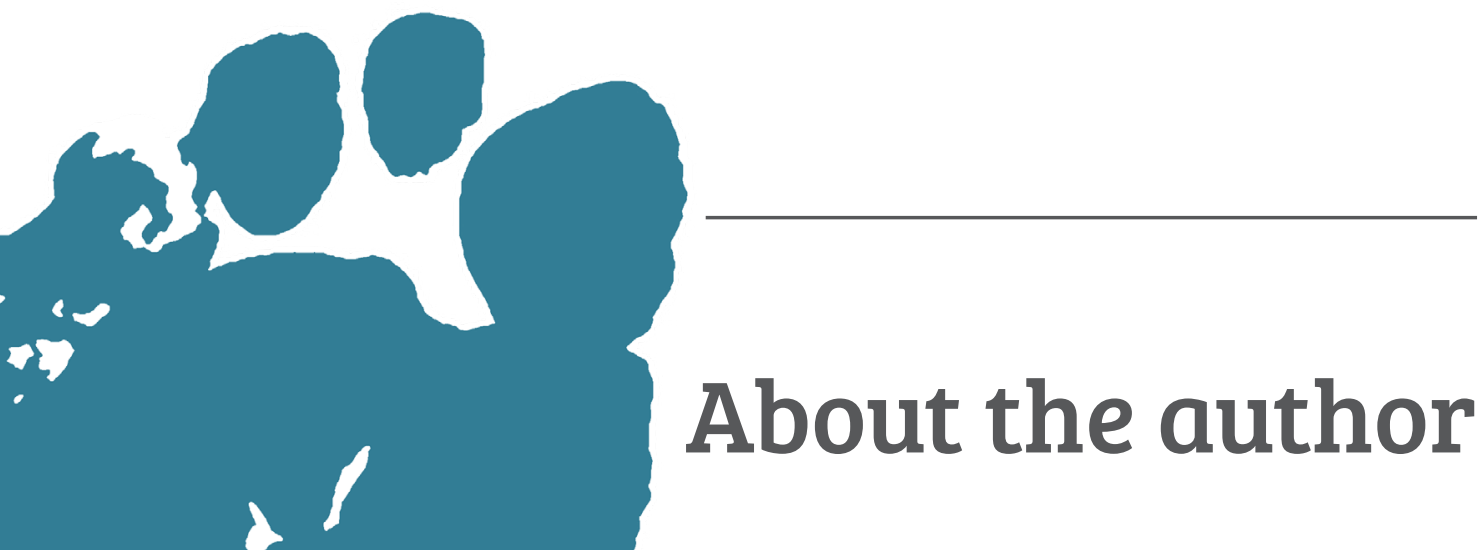

$x$

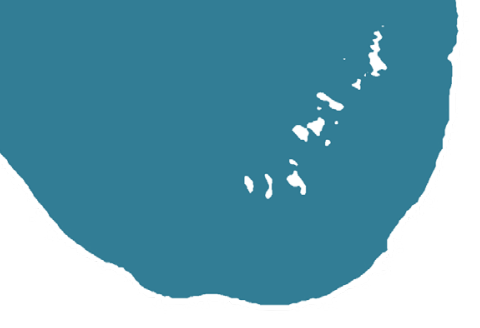


Kelly Stewart was born in Eindhoven on a misty November $7^{\text {th }} 1988$. Graduating secondary school (Lorentz Casimir Lyceum, Eindhoven) in 2006, she moved to Ireland and travelled Latin America and Australia. To great relief of her mother, she resumed her education in 2009.

Kelly obtained her bachelor degree in Nutrition and Health in 2012 in Wageningen. Her bachelor thesis was entitled "The efficacy of chondroitin for the prevention and treatment of osteoarthritis in athletes". She then returned south to start her master education at Maastricht University, obtaining her MSc. degree in Sports and Physical Activity Interventions in 2013 for which she received the distinction cum laude. Her master thesis took place at CIRO in Horn, the Netherlands, under supervision of Prof. Annemie Schols and Jessie Meis. Her thesis, entitled "Maintenance of healthy lifestyle behaviours after a 4 month pulmonary rehabilitation programme in COPD patients" was published in the Journal of the American Medical Directors Association in 2014.

With the new year of 2014 she started her PhD track at the Department of Complex Genetics under the supervision of Prof. Maurice Zeegers, Dr. Anke Wesselius and Prof. Annemie Schols. Her work focused on (1) disease risk prediction based on genetic profiling and lifestyle factors, (2) evaluating issues surrounding direct-to-consumer genetic testing and (3) development of a new genetic testing service: Health Potential. The research she performed in this time is presented in the current thesis.

After four years of studying the topic, she has finally supplied a saliva sample for a genetic test. 
About the author 


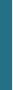


Stewart KFJ, Meis JJ, van de Bool C, Janssen DJ, Kremers SP, Schols AM. Maintenance of a physically active lifestyle after pulmonary rehabilitation in patients with COPD: a qualitative study toward motivational factors. Journal of the American Medical Directors Association. 2014; 15(9):655-64.

Al-Zalabani AH*, Stewart KFJ*, Wesselius A, Schols AM, Zeegers MP. Modifiable risk factors for the prevention of bladder cancer: a systematic review of meta-analyses. European journal of epidemiology. 2016; 31(9):811-51.

Stewart KFJ, Wesselius A, Schreurs MA, Schols AM, Zeegers MP. Behavioural changes, sharing behaviour and psychological responses after receiving direct-to-consumer genetic test results: a systematic review and meta-analysis. Journal of community genetics. 2017; 9(1):1-18.

Weyerstraß J, Stewart K, Wesselius A, Zeegers M. Nine genetic polymorphisms associated with power athlete status-A Meta-Analysis. Journal of science and medicine in sport. 2018; 21(2):213-220.

Stewart KFJ, Wesselius A, Schols A M W J, Zeegers MP. Stages of behavioural change after direct-to-consumer disease risk profiling: study protocol of two integrated controlled pragmatic trials. Trials Journal. 2018; 19(1):240

Elahi IRN*, Katib AA*, Alamri AG*, Halawani AT*, Alsindi NM*, Almatrafi MA*, Al-Zalabani AH, Wesselius A, Stewart KFJ. The Association Between Soft Drinks Consumption and Asthma: A Systematic Review and Meta-Analysis. (Submitted)

Mount SW*, Stewart KFJ*, Wesselius A, Zeegers MP, Schols AMWJ. Genetic, lifestyle, and environmental factors associated with development of chronic obstructive pulmonary disease. (Submitted)

Stewart KFJ, Kokole D, Wesselius A, Schols AMWJ, Zeegers MP, Van Osch LADM. Factors associated with acceptability, consideration and intention of uptake of direct-toconsumer genetic testing: a survey study. (Accepted by Public Health Genomics)

* Joint first authorships 


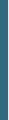

